Estimativas de erro para discretizações do Laplaciano por métodos de volumes finitos em malhas esféricas geodésicas icosaédricas

Leonardo Andrés Poveda Cuevas

\author{
TESE APRESENTADA \\ $\mathrm{AO}$ \\ Instituto DE MATEMÁticA E EstatísticA \\ DA \\ Universidade DE SÃO PAUlo \\ PARA \\ OBTENÇÃO DO TÍTULO \\ $\mathrm{DE}$ \\ DOUTOR EM CiênCIAS \\ Programa: Matemática Aplicada \\ Orientador: Prof. Dr. Saulo Rabello Maciel de Barros \\ Coorientador: Prof. Dr. Pedro da Silva Peixoto
}

Durante o desenvolvimento deste trabalho o autor recebeu auxílio financeiro da CAPES

São Paulo, janeiro de 2021 


\title{
Estimativas de erro para discretizações do Laplaciano por métodos de volumes finitos em malhas esféricas geodésicas icosaédricas
}

\author{
Esta versão da tese contém as correções e alterações sugeridas \\ pela Comissão Julgadora durante a defesa da versão original do trabalho, \\ realizada em 22/01/2021. Uma cópia da versão original está disponível no \\ Instituto de Matemática e Estatística da Universidade de São Paulo.
}

Comissão Julgadora:

- Prof. Dr. Saulo Rabello Maciel de Barros (Presidente) - IME-USP

- Prof. Dr. Marcone Corrêa Pereira - IME-USP

- Prof. Dr. André Pierro de Camargo - UFABC

- Prof. Dr. Marcus Antonio Mendonça Marrocos - UFABC

- Prof. Dr. Gaetano Siciliano - IME-USP 


\section{Agradecimentos}

Quero expressar um agradecimento a meu orientador, o Professor Saulo, pela orientação, a paciência e as valiosas discussões durante o desenvolvimento deste trabalho. Lembrarei dele a ideia de que no final do doutorado eu deveria me tornar uma pessoa com sentido crítico. Ao Professor Pedro Peixoto pelo acompanhamento, as discussões e as sugestões da implementação numérica requerida no processo do trabalho. Tenho que agradecer também aos professores do IME pelas disciplinas cursadas. Agradeço aos membros da banca, por aceitarem o convite, pela leitura e as distintas sugestões para o trabalho.

Eu não teria conseguido chegar até aqui sem a companhia das pessoas mais importantes da minha vida. Agradeço principalmente a minha esposa Paola, quem com seu amor, paciência e compreensão incondicional contribuiu em me animar sempre em seguir em frente, além de incentivar diversas ideias que surgiram nas nossas extraordinárias discussões no caminho à Universidade e a ajuda com a revisão da tese. Aos meus pais, Aura Rosa e Fredy, que sempre me apoiaram e incentivaram em todos os meus caminhos pessoais e profissionais. Aos meus irmãos Jackson e Alejandro e a minha irmã Laura, pela confiança, companhia, apoio abrangente e por compartilhar aquelas risadas nos diferentes momentos das nossas vidas. A minha sogra Irene que me apoiou e acompanhou com as suas bênçãos e orações cada dia. À minha cunhada Jhohana (in memoriam) e aos meus cunhados Vladimir e Gustavo por desejar sempre o melhor para mim e para Paola.

Às minhas colegas do LabMap: Simone Brito, pela sua a amizade, a generosidade e a ajuda nos momentos diversos, Larissa Sartori, pela amizade, as conversas de ânimo e o suporte no caminho. Demonstro igualmente minha gratidão pela amizade que sempre me ofereceram: Sandra Suárez, Vanessa Borges, Vanessa Steindorf, Laura Forero, Bruna Cassol e Ana "Anita" Rojas. Agradeço também aos meus colegas do IME: Carolina Torres, Diana, Jerusa, Sueni, Pollyanna, Luana, Camilo Angulo, Edwin Múrcia, Diego Ruge, Gregório, Lucas, Allan e German Benitez.

Destaco o enorme apoio recebido do meu grande amigo da faculdade e colega no IME, Alirio Gomez, nos momentos difíceis e que após vários bate-papos acadêmicos e filosóficos sobre os desafios da matemática, sempre mostrou um entusiasmo que indiretamente contribuiu no desenvolvimento desta pesquisa. Agradeço também ao Carlos Wilson e a Adriana por todos aqueles bons momentos vividos em São Paulo.

Agradeço à Professora Carla Columbano do IQ-USP, a orientadora da Paola, por permitir invadir os espaços da sala de estudo e pelo seu apoio financeiro a Paola, que indiretamente me beneficiou nestes últimos anos.

Agradeço à CAPES o apoio financeiro. Também a Comissão de Monitoria IME-USP.

Finalmente, quero agradecer a todos os funcionários, em especial as funcionárias do café, que fazem do IME um ótimo ambiente de trabalho. Além, aos funcionários da BIME, aos funcionários do SAS, aos funcionários do CRUSP, aos funcionários do CEPEUSP, aos funcionários da segurança e da limpeza e aos funcionários dos Restaurantes universitários da USP. 


\section{Resumo}

CUEVAS, Leonardo A. P. Estimativas de erro para discretizações do Laplaciano por métodos de volumes finitos em malhas esféricas geodésicas icosaédricas. 2021. 115 f. Tese (Doutorado) - Instituto de Matemática e Estatística, Universidade de São Paulo, São Paulo, 2021.

A presente tese concentra-se no estudo de estimativas de erro das soluções aproximadas da discretização do Laplaciano pelo Método clássico de Volumes Finitos na esfera unitária $\mathbb{S}^{2}$. Inicialmente e conforme a literatura, estabelecemos a boa colocação do problema de Poisson em $\mathbb{S}^{2}$. Em seguida, apresentamos a construção recursiva usual das malhas esféricas geodésicas icosaédricas (NOPT) e esboçamos propriedades geométricas essenciais como o principio de dualidade VoronoïDelaunay e as otimizações SCVT e HR95, sendo que a precisão da soluções aproximadas depende da qualidade da malha.

Definida a estrutura, introduzimos uma discretização do Laplaciano via um Método clássico de Volumes Finitos (MVF). De forma geral, ao usar este método, frequentemente aparece uma perda da consistência causada pela não uniformidade da malha; mas isso não implica necessariamente em perda de convergência. Esse fato foi a motivação principal para o desenvolvimento deste trabalho. Nesse sentido, analisamos taxas de convergência através de uma abordagem mais tradicional, que consiste em reescrever o MVF como um Método de Elementos Finitos Lineares (MEFL) e usar a teoria de aproximação disponível dos MEFL. Aqui, mostramos estimativas clássicas a priori de primeira ordem nas normas $H^{1}$ e $L^{2}$ de forma direta.

O principal resultado deste trabalho são estimativas nas normas $W^{1, \infty}$ e $L^{\infty}$ com ordem sublinear em relação ao tamanho de malha. Tais estimativas são obtidas através do uso de funções de Green regularizadas em $\mathbb{S}^{2}$, combinadas com a relação entre o método de volumes finitos com elementos finitos. No caso de uso de uma malha SCVT a convergência nestas normas é superlinear (quase quadrática). Apresentamos exemplos numéricos confirmando as estimativas do erro, evidenciando que ao menos tais ordens de convergência são atingidas.

Palavras-chave: Laplaciano, malha esférica icosaédrica, volumes finitos, estimativas de erro $a$ priori, estimativas pontuais, estimativas em norma uniforme. 


\section{Abstract}

CUEVAS, Leonardo A. P. Error estimates for discretizations of the Laplacian by finite volume methods on spherical geodesic icosahedral grids. 2021. $115 \mathrm{f}$. Tese (Doutorado) Instituto de Matemática e Estatística, Universidade de São Paulo, São Paulo, 2021.

In this work, we focus on the study of error estimates of the approximate solutions of a Poisson equation on the unit sphere $\mathbb{S}^{2}$, discretized by a classical Finite Volume Method (FVM). Initially and according to the literature, we establish the well-posedness of the problem. Next, we present the usual recursive construction of the spherical icosahedral geodesic grids (NOPT). We sketch essential geometric properties as the duality principle of Voronoï-Delaunay and SCVT and HR95 optimizations, since the precision of approximate solutions depends on the quality of the grid.

The usual finite volume discretization of the Laplacian on icosahedral spherical grids presents a loss of consistency, caused by non-uniformity of the grids. However, this does not necessarily imply in a degradation of convergence. This fact was the main motivation for the development of this work. In this sense, we analyse convergence rates through a more traditional approach, which consists on rewriting the FVM as a Linear Finite Element Method (LFEM) and use the available LFEM approximation theory. Here we present some classical a priori first order estimates in $H^{1}$ and $L^{2}$-norms.

The main contribution of this work are estimates in the $W^{1, \infty}$ and $L^{\infty}$-norms with sub-linear order concerning the mesh size. The proofs employ regularized Green's functions on $\mathbb{S}^{2}$, combined with the finite element formulation for the finite volume method. In the case of the SCVT grid we obtain super-linear (almost quadratic) convergence estimates. Numerical examples evidence that the convergence is at least as good as predicted.

Keywords: Laplacian, finite volume, spherical icosahedral grids, a priori error estimates, pointwise estimates, uniform error estimates. 


\section{Sumário}

$\begin{array}{ll}\text { Lista de Abreviaturas } & \text { ix }\end{array}$

Lista de Símbolos $\quad$ xi

Introdução $\quad$ Xv

1 Preliminares $\quad 1$

1.1 Propriedades geométricas em $\mathbb{S}^{2} \ldots \ldots \ldots \ldots \ldots \ldots \ldots$

1.2 Espaços de funções . . . . . . . . . . . . . . . . . . . . . . . . 2

1.3 Espaços de Sobolev em $\mathbb{S}^{2} \ldots \ldots \ldots \ldots \ldots \ldots \ldots \ldots \ldots$

1.3 .1 Espaço quociente . . . . . . . . . . . . . . . . . 5

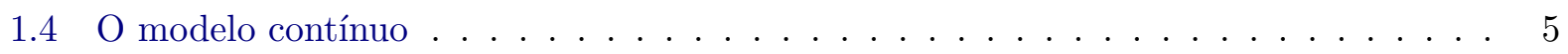

1.4.1 Uma formulação variacional . . . . . . . . . . . . . . . . 6

2 Malhas esféricas geodésicas icosaédricas $\quad 9$

2.1 Estruturas de malhas na esfera . . . . . . . . . . . . . . . . . 9

2.2 Malhas esféricas icosaédricas . . . . . . . . . . . . . . . . . 10

2.2 .1 Construção recursiva . . . . . . . . . . . . . . . . . . . 10

2.2 .2 Decomposição Voronoï-Delaunay . . . . . . . . . . . . . . . . . . . . . . . 12

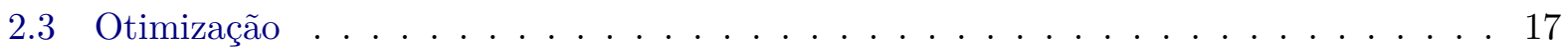

2.3.1 Malha esférica centroidal de Voronoï (SCVT) . . . . . . . . . . . . . 17

2.3.2 Proposta de Heikes \& Randall (HR95) . . . . . . . . . . . . . . . . . . . . 19

3 Discretização do Laplaciano $\quad 21$

3.1 Métodos dos volumes finitos . . . . . . . . . . . . . . . . . . 21

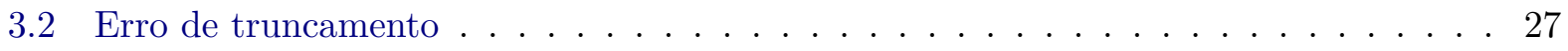

3.2 .1 Experimentos numéricos . . . . . . . . . . . . . . . . 28

3.3 Relação MVF-MEFL e estabilidade . . . . . . . . . . . . . . . . . . . . . . . 29

3.3 .1 Correspondência geométrica . . . . . . . . . . . . . . . . . 30

3.3 .2 Espaços de aproximação . . . . . . . . . . . . . . . . . . . 32

3.3 .3 Estabilidade. . . . . . . . . . . . . . . . . . . . 37

3.3 .4 Estimações do erro geométrico . . . . . . . . . . . . . . . . . 39

4 Estimativas a priori do erro $\quad 45$

4.1 Estimativas clássicas na $H^{1}$ e $L^{2}$-norma . . . . . . . . . . . . . . . . . 45

4.2 Estimativas na max-norma . . . . . . . . . . . . . . . . 52

$4.2 .1 \quad$ Funções de Green em $\mathbb{S}^{2} \ldots \ldots \ldots$. . . . . . . . . . . . . . . . . 52 


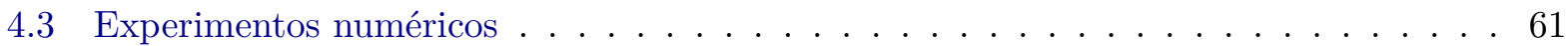

$\begin{array}{ll}\text { Conclusões } & 65\end{array}$

$\begin{array}{ll}\text { A Erro de consistência } & 67\end{array}$

A.1 Uma condição de consistência fraca . . . . . . . . . . . . . . . . . . . . . . 67

A.1.1 Prova da Proposição 3.5 . . . . . . . . . . . . . . . . . . . . . 69

A.1.2 Prova da Proposição $3.6 \ldots \ldots \ldots$. . . . . . . . . . . . . 72

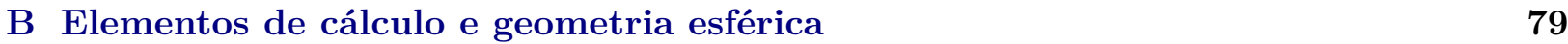

B.1 Fórmula de Taylor e desigualdade de valor médio . . . . . . . . . . . . . . . . 79

B.2 Coordenadas geográficas . . . . . . . . . . . . . . . . . . . 79

B.3 Distâncias geodésicas . . . . . . . . . . . . . . . . . 80

B.4 Áreas geodésicas . . . . . . . . . . . . . . . . . . 80

B.5 Mudança de variável . . . . . . . . . . . . . . . . . . . 80

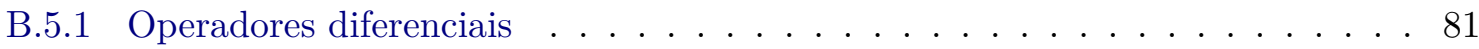

$\begin{array}{ll}\text { C Elementos de álgebra linear } & \mathbf{8 3}\end{array}$

C.1 Considerações técnicas . . . . . . . . . . . . . . . . . . . 83

C.2 Existência da solução discreta . . . . . . . . . . . . . . . . . . . . 83

C.3 Método de Gauss-Seidel SOR (Succesive over relaxation) . . . . . . . . . . . . . . 84

$\begin{array}{ll}\text { Referências Bibliográficas } & 87\end{array}$ 


\section{Lista de Abreviaturas}

EDP Equação Diferencial Parcial (Partial Differential Equation)

NOPT Malha esférica geodésica icosaédrica não otimizada

SCVT Malha esférica geodésica icosaédrica otimizada com o critério de Du et al. (2003a) (Spherical Centroidal Voronoï Tessellelation)

HR95 Malha esférica geodésica icosaédrica otimizada com o critério de Heikes e Randall (1995b)

MVF Método de Volumes Finitos (Finite Volumes Method)

MDF Método de Diferenças Finitas (Finite Differences Method)

MEF Método de Elementos Finitos (Finite Elements Method)

MEFL Método de Elementos Finitos Lineares (Linear Finite Elements Method)

MEVF Método de Elementos de Volumes Finitos (Finite Volume Element Method) 


\title{
Lista de Símbolos
}

\author{
Geometría \\ $\mathbb{N}$ \\ Conjunto de números naturais \\ $\mathbb{R}, \mathbb{R}^{2}, \mathbb{R}^{3}$ \\ Reta, plano e espaço real \\ $\mathbb{R}_{+}$ \\ Conjunto de números reais positivos \\ $\mathbb{S}^{2}$ \\ Esfera unitária \\ $\mathrm{x}, \mathrm{y}, \mathrm{z}$ \\ Pontos na esfera unitária \\ $x_{1}, x_{2}, x_{3}$ \\ $\mathrm{d}(\cdot, \cdot)$ \\ Coordenadas do ponto x no espaço $\mathbb{R}^{3}$ \\ $|\cdot|$ \\ $\|\cdot\|_{\mathbb{R}^{3}},\|\cdot\|$ \\ $\widetilde{\mathrm{V}}$ \\ $\operatorname{Int}(\widetilde{\mathrm{V}})$ \\ $\operatorname{cl}(\widetilde{\mathrm{V}})$ \\ $\partial \widetilde{\mathrm{V}}$ \\ $\widetilde{\Gamma}$ \\ Distância geodésica \\ Módulo de um número real \\ Norma Euclidiana no $\mathbb{R}^{3}$ \\ Região esférica \\ Interior da região esférica $\widetilde{\mathrm{V}}$ \\ Fecho da região esférica $\widetilde{V}$ \\ Fronteira ou contorno da região esférica $\widetilde{V}$ \\ Geodésica em $\mathbb{S}^{2}$ \\ $S(\cdot)$ \\ Área superficial esférica \\ $\mathrm{m}(\cdot)$ \\ Comprimento geodésico \\ $\operatorname{Card}(\cdot)$ \\ Cardinalidade de conjuntos
}

Espaços de funções

$\partial_{i}$

Derivada parcial na coordenada $x_{i}$

$\nabla$

Gradiente usual no $\mathbb{R}^{3}$

$\nabla_{s}$

Gradiente ou componente tangencial na esfera $\mathbb{S}^{2}$

$\partial_{s, i}$

Derivada parcial tangencial na coordenada $x_{i}$

$\nabla_{s}^{\alpha}$

Operador diferencial tangencial de ordem $\alpha$

$-\Delta_{s}$

Laplaciano na esfera

$\mathbb{T}_{\mathbb{S}^{2}, \mathrm{x}}$

Plano tangente à $\mathbb{S}^{2}$ no ponto x

$\overrightarrow{\mathrm{n}}_{\mathbb{S}^{2}, \mathrm{x}}$

Vetor normal unitário à esfera $\mathbb{S}^{2}$ no ponto x

$\overrightarrow{\mathrm{n}}_{\mathrm{x}, \widetilde{\mathrm{V}}_{i}}$

$\overrightarrow{\mathrm{n}}_{\mathrm{x}, \widetilde{\Gamma}_{i j}}$

Vetor normal unitário à fronteira $\partial \widetilde{\mathrm{V}}_{i}$ contido em $\mathbb{T}_{\mathbb{S}^{2}, \mathrm{x}}$ no ponto $\mathrm{x}$

Vetor normal unitário à aresta dual $\widetilde{\Gamma}_{i j}$ contido em $\mathbb{T}_{\mathbb{S}^{2}, \mathrm{x}}$ no ponto $\mathrm{x}$

$\overrightarrow{\mathrm{n}}_{\mathrm{x}, \widetilde{\mathrm{T}}_{i j k}}$

Vetor normal unitário à triângulo $\widetilde{\mathrm{T}}_{i j k}$ contido em $\mathbb{T}_{\mathbb{S}^{2}, \mathrm{x}}$ no ponto $\mathrm{x}$

$C^{k}(\cdot)$

Espaço de funções $k$-vezes diferenciáveis

$(\cdot, \cdot)$

Produto escalar

$W^{m, p}\left(\mathbb{S}^{2}\right)$

$\|\cdot\|_{W^{m, p}\left(\mathbb{S}^{2}\right)}$

Espaços de Sobolev definido em $\mathbb{S}^{2}$ com $m \geq 0$ e $1 \leq p \leq \infty$

$H^{m}\left(\mathbb{S}^{2}\right)$

Norma no espaço $W^{m, p}\left(\mathbb{S}^{2}\right)$

$\|\cdot\|_{H^{m}\left(\mathbb{S}^{2}\right)}$

Espaço de Sobolev-Hilbert definido em $\mathbb{S}^{2}$

$H_{Q}^{1}\left(\mathbb{S}^{2}\right)$

Norma no espaço $H^{m}\left(\mathbb{S}^{2}\right)$

Espaço quociente definido em $\mathbb{S}^{2}$ 


\section{Aproximação da esfera}

$\mathbf{S}_{h}$

$\mathcal{T}_{h}$

$\widetilde{\mathcal{T}}_{h}$

$\widetilde{\mathcal{V}}$

$\mathrm{x}_{i}$

$N$

$\mathrm{S}_{N}$

$\ell$

$\tilde{\mathrm{V}}_{i}$

$\widetilde{\mathrm{T}}_{i j k}$

$h$

$\mathcal{P}$

$\widetilde{U}_{h}$

$\widetilde{V}_{h}$

$\Pi_{h}$

$\widetilde{\Pi}_{h}$

$\mathrm{I}_{h}$

$\mathcal{A}(\cdot, \cdot)$

$\mathcal{A}_{h}(\cdot, \cdot)$

$\mathcal{A}_{h}^{d}(\cdot, \cdot)$

$\langle\cdot, \cdot\rangle_{h}$

$\|\cdot\|_{n, h}$

$\xi_{h}$

$\widetilde{\xi}_{h}$

$\varepsilon_{h}$

$G(\mathrm{x}, \mathrm{y}), G^{\mathrm{y}}(\mathrm{x})$

$\hat{G}^{\mathrm{y}}(\mathrm{x})$

$\hat{G}_{h}^{\mathrm{y}}(\mathrm{x})$

$\delta(\mathrm{x}-\mathrm{y}), \delta^{\mathrm{y}}(\mathrm{x})$
Poliedro de aproximação à esfera

Decomposição triangular plana-primal

Decomposição esférica primal ou Delaunay

Decomposição esférica dual de Voronoï

Ponto de malha primal ou gerador de malha dual

Número de vértices da malha

Conjunto de vértices $\mathrm{x}_{i}$

Nível de recursão ou refinamento de malha

Região esférica de Voronoï associada ao vértice $\mathrm{x}_{i}$

Triângulo esférico com vértices $\mathrm{x}_{i}, \mathrm{x}_{j}$ e $\mathrm{x}_{k}$

Parâmetro de malha em relação à distância geodésica máxima

Projeção radial na esfera unitária $\mathbb{S}^{2}$

Espaço de funções lineares por partes associado a $\widetilde{\mathcal{T}}_{h}$

Espaço de funções constantes por partes associado a $\widetilde{\mathcal{V}}$

Interpolador linear de $\widetilde{U}_{h}$

Interpolador linear de $\widetilde{V}_{h}$

Interpolador linear de transferência entre os espaços $\widetilde{U}_{h}$ e $\widetilde{V}_{h}$

Forma variacional para o MEFL

Forma variacional para o MVF

Forma variacional discreta para o MVF

Produto escalar discreto

norma discreta para $n=0,1$ ou $\infty$

Erro de truncamento

Erro de truncamento fraco

Erro da soluções

Função de Green com singularidade em y $\in \mathbb{S}^{2}$

Função de Green discreta

Aproximação por MEFL da função $\hat{G}^{y}$

Função delta de Dirac 


\section{Introdução}

Nessa tese estudamos a taxa de convergência das soluções aproximadas por uma discretização do Laplaciano baseada nos Métodos clássicos de Volumes Finitos nas malhas geodésicas esféricas icosaédricas da esfera unitária. Revisemos como é feita a construção das estimativas.

O estudo de soluções numéricas das Equações Diferenciais Parciais (EDPs) na esfera é uma área de pesquisa ativa na comunidade científica. O interesse está concentrado em uma ampla variedade de aplicações tais como: geometria, dinâmica dos fluidos, modelagem de materiais, ciências ambientais e biológicas, processamento de imagens entre outras. Neste trabalho, vamos considerar a equação de Poisson na esfera unitária $\mathbb{S}^{2}$. Consideramos o problema modelo,

$$
-\Delta_{s} u(\mathrm{x})=f(\mathrm{x}), \quad \text { para cada } \mathrm{x} \in \mathbb{S}^{2}
$$

onde $-\Delta_{s}$ representa o operador de Laplace-Beltrami (o Laplaciano em superfícies) que trabalha como uma generalização do operador de Laplace padrão (Demlow, 2009; Demlow e Dziuk, 2007; Dziuk e Elliott, 2013). O problema de Poisson está presente em diversas aplicações, como por exemplo os modelos da previsão do tempo globais (Scott et al., 2016; Tomita et al., 2001; Vreugdenhil, 2013). De particular interesse, essas aplicações são tradicionalmente resolvidas mediante modelos numéricos empregando uma discretização da superfície da terra em um sistema de coordenadas esféricas.

Nos últimos 30 anos, a pesquisa tem sido direcionada a gerar malhas apropriadas de $\mathbb{S}^{2}$. Isso virou um requisito essencial para os modelos numéricos, dado que a precisão e a acurácia das soluções aproximadas, junto à eficiência com a qual são obtidas, dependem de parâmetros da qualidade da malha; essencialmente a uniformidade, a regularidade e a quantidade de células da malha. Nesse sentido, foi surgindo um interesse nas malhas geradas a partir de poliedros regulares inscrito em $\mathbb{S}^{2}$ (Giraldo, 1997; Heinze e Hense, 2002; Randall et al., 1998; Ronchi et al., 1996). Entre as mais relevantes e que trataremos neste trabalho, estão as formadas a partir de um icosaedro regular conhecida popularmente como a malha esférica geodésica icosaédrica (Giraldo, 1997; Heikes e Randall, 1995a; Ii e Xiao, 2010; Tomita et al., 2001). Esse tipo de estrutura aparece inicialmente nos trabalhos de Sadourny et al. (1968) e Williamson (1968), mas ganharam uma melhor acolhida com as propostas de Baumgardner e Frederickson (1985) e Heikes e Randall (1995a), devido principalmente à distribuição uniforme dos vértices na superfície esférica.

Além de ser gerada de forma simples, de acordo com Augenbaum e Peskin (1985); Renka (1997) e Du e Wang (2006), a malha esférica geodésica icosaédrica proporciona entre outras coisas, condições geométricas propicias para a discretização de EDPs. Em princípio, dado que essa malha é composta unicamente por triângulos geodésicos, ela satisfaz a condição de Delaunay, i.e., tem a capacidade de maximizar o menor ângulo de todos os seus triângulos (Augenbaum e Peskin, 1985; Gärtner e Kamenski, 2019; Renka, 1997), e portanto, define uma decomposição primal de 
Delaunay em $\mathbb{S}^{2}$. Usando o princípio de dualidade Voronoï-Delaunay (Gärtner e Kamenski, 2019; Hjelle e Dæhlen, 2006) existe uma e somente uma decomposição dual de Voronoï, cujos vértices são os circuncentros dos triângulos da decomposição primal. Neste trabalho, a malha esférica icosaédrica será denominada como decomposição Voronoï-Delaunay em $\mathbb{S}^{2}$. Conforme já reportado por Williamson et al. (1992) e Heikes e Randall (1995b), a não uniformidade dessa malha dificulta a análise de certos operadores discretos. Uma primeira forma de lidar com esse inconveniente é invocar processos de otimização na qualidade da malha (Du et al., 2003a; Heikes e Randall, 1995a,b; Miura e Kimoto, 2005; Satoh, 2013; Tomita et al., 2001, 2002; Wang e Lee, 2011). Por simplicidade da apresentação, consideramos só duas dessas optimizações, que são: o critério da SCVT (Spherical Centroidal Voronoï Tessellation), desenvolvido por Du et al. (2003a), que avalia a distância entre os vértices (centros) e os centroides das células duais, e o critério da HR95 proposto por Heikes e Randall (1995b) que avalia as distâncias dos pontos médios das arestas primal/dual. Vale ressaltar que a abordagem desta tese não pretende aprimorar esses critérios nem desenvolver critérios novos. Apenas enfatizamos que as malhas otimizadas possuem suas próprias características geométricas e peculiaridades com relação à estimação dos erros de discretização e de convergência de soluções discretas. Além disso, até o presente, nenhuma técnica conhecida se mostrou eficaz na otimização simultânea de parâmetros da malha Miura e Kimoto (2005); Peixoto (2013).

Descrita a infraestrutura geométrica de $\mathbb{S}^{2}$, nos concentramos no estudo do comportamento das soluções aproximadas pelos Métodos de Volumes Finitos (MVF) para a equação de Poisson. Em primeiro lugar, os MVF são altamente potentes para modelagem numérica de uma ampla variedade de problemas físicos, dado que são bastante flexíveis na manipulação de geometrias complicadas que levam a esquemas robustos em comparação com os Métodos de Diferenças Finitas (MDF). Além de ser mais simples de implementar e gerar resultados satisfatórios em relação ao custo computacional, uma característica essencial é a conservação local dos fluxos numéricos, comparados esta vez com os Métodos de Elementos Finitos (MEF) (Eymard et al., 2000, 2001; Hermeline, 2000; LeVeque et al., 2002).

A construção do MVF em malhas tipo Voronoï-Delaunay é baseada na integração da equação em cada célula da decomposição dual. Através do teorema de Gauss é obtida uma formulação integral dos fluxos na fronteira da célula. Posteriormente, essa forma integral dos fluxos é aproximada no ponto médio de cada aresta da fronteira. Em seguida, essa aproximação dos fluxos é discretizada via um esquema de diferença finita centrada. Apesar de ser um método numérico robusto, comumente se observa uma perda na ordem de consistência causada pela não uniformidade da decomposição Voronoï-Delaunay. Não obstante, técnicas de análise de erro das diferenças finitas feitas por Barbeiro (2009); Bouche et al. (2005); Despres (2004) e Pascal (2007), mostraram que nas malhas não uniformes, a degradação no erro de consistência não necessariamente implica na perda de convergência das soluções. Em outras palavras, a convergência das soluções pode-se dar mesmo quando o erro de consistência exibe ordem inferior ou em certos casos não converge em absoluto. Este fenômeno de supraconvergência vem sendo detalhadamente investigado há vários anos e mostra que a consistência não é necessariamente um bom indicador da ordem de convergência dos operadores discretos nesse caso (Diskin e Thomas, 2010; Droniou, 2014; Kreiss et al., 1986; Manteuffel e White, 1986; Pascal, 2007) . Por exemplo, convergência em norma $L^{2}$ no plano têm sido bem estabelecida por Coudière et al. (1999); Eymard et al. (2001); Gallouët et al. (2000); Herbin (1995); Mishev (1998) e Eymard et al. (2006). Em Du et al. (2003b) são apresentadas 
extensões para problemas de convecção difusão em $\mathbb{S}^{2}$. Nestes trabalhos mencionados mostra-se ordem de convergência um na norma $L^{2}$ sob condições de regularidade da malha, neste caso ser quase-uniforme (Cai, 1990; Ciarlet, 2002; Mishev, 1998).

Adicionalmente, Heikes e Randall (1995b) e Ringler (2003) realizaram experimentos numéricos de MVF para decomposições Voronoï-Delaunay em $\mathbb{S}^{2}$ e verificaram que, embora haja inconsistência no MVF, o método é convergente. Porém, esse estudo é empírico e não prova matematicamente que há convergência das soluções aproximadas, em especial na norma uniforme, para a qual não conhecemos estimativas de convergência na literatura. Portanto, obter estimativas a priori para o MVF nesta norma ainda é uma tarefa desafiadora, sendo a motivação principal para o desenvolvimento desta tese. Uma possível abordagem para obter estimativas na norma do máximo foi descrita por Faille (1992) para problemas no plano usando condições de Dirichlet na fronteira em malhas retangulares. $\mathrm{O}$ autor assume condições de regularidade na solução exata, e que o MVF conservativo satisfaz uma condição de consistência fraca, que junto da hipótese de estabilidade, garante a convergência das soluções aproximadas. Porém, esse estudo não determina qual é a ordem de convergência. Seguindo essa análise nós tentamos aplicar de forma preliminar uma extensão à $\mathbb{S}^{2}$, mostrando unicamente que o MVF é fracamente consistente na max-norma sob condições na decomposição Voronoï-Delaunay. Porém, a convergência não pode ser garantida dado que algumas das hipóteses de estabilidade não foram verificadas. Não nos parece simples determinar, por meio de técnicas de diferenças finitas, uma estimativa de convergência dos MVF para decomposições Voronoï-Delaunay em $\mathbb{S}^{2}$ na norma do máximo.

Optamos portanto por seguir uma outra abordagem, descrita amplamente na literatura (Ewing et al., 2002; Li et al., 2000; Lin et al., 2013), usada na obtenção de estimativas em outras normas. A ideia chave consiste em reescrever o MVF como um Método de Elementos Finitos Lineares (MEFL) e empregar os procedimentos padrão dos MEF para as estimativas de convergência. Os MEF são também métodos populares para encontrar soluções de EDPs na forma variacional e o seu potencial é devido à sua extensa base teórica do princípio variacional acoplado a uma conveniente aproximação dos espaços funcionais (Brenner e Scott, 2007; Ciarlet, 2002). Além disso, há progressos na teoria de aproximações dos MEF em superfícies (Bonito e Demlow, 2019; Demlow, 2009; Demlow e Dziuk, 2007; Dziuk, 1988; Dziuk e Elliott, 2013; Kröner, 2017) e parcialmente dos MVF para decomposições duais não Voronoï (Ju e Du, 2009; Ju et al., 2009). Em nosso trabalho, focamos nas estimativas de erro do MVF para decomposições Voronoï-Delaunay em $\mathbb{S}^{2}$.

Para começar, consideramos um espaço de elementos finitos lineares associado à descomposição primal de Delaunay. Agora, dado o princípio de dualidade na decomposição, o espaço de volumes finitos adequado é o gerado pela descomposição dual de Voronoï, cujos elementos são funções constantes por pedaços em cada uma das suas células. Assim, a solução aproximada pelo MVF é linear por partes associada à decomposição primal. Em seguida, definimos formas variacionais contínua e discreta para o $\mathrm{MVF}$, com o propósito de estabelecer a estabilidade. Usando a estabilidade junto com relação entre as formas variacionais do MVF e MEFL, estabelecemos estimativas de convergência de primeira ordem nas normas $H^{1}$ e $L^{2}$ de forma direta, diferentemente das estimativas já apresentadas nos trabalhos de Eymard et al. (2001); Gallouët et al. (2000); Mishev (1998) e Du et al. (2003b).

Outros estudos utilizam decomposições que não são Voronoï-Delaunay e determinam estimativas 
de convergência de ordem dois nestas normas para problemas no plano, como as dadas por Chou e Li (2000); Chou e Ye (2007); Chou et al. (2003); Jianguo e Shitong (1998); Li et al. (2000); Wu e Li (2003) e para superfícies por Ju e Du (2009) e Ju et al. (2009). Destacamos que a ordem das estimativas é obtida com o uso explicito dos baricentros dos triângulos e uma exigência extra na regularidade da solução exata, que é excessiva em comparação à pedida nos MEFL. Entretanto, Ewing et al. (2002) estabeleceu uma hipótese suficiente para diminuir essa regularidade na solução exata, e impor um requisito mínimo de regularidade no termo fonte. É importante notar que a construção das estimativas descritas acima não podem ser estendidas à decomposição VoronoïDelaunay sem algumas considerações adicionais, como no caso mostrado por Du e Ju (2005), onde a estimativa de ordem quadrática na norma $L^{2}$ depende do critério de optimização da SCVT e da regularidade excessiva na solução exata.

Baseado nessa análise, aqui mostramos uma modificação da prova aplicando o fundamento de Ewing et al. (2002), i.e., diminuindo a regularidade na solução exata e impondo uma mínima regularidade no termo fonte, e assim obter a ordem de convergência desejada. Concluímos, o mesmo que Du e Ju (2005), a prova não pode ser estendida para decomposições Voronoï-Delaunay em geral devido ao uso explícito do critério da SCVT ou para a $H^{1}$-norma já que existe um comportamento de superconvergência na aproximação dos gradientes. De modo geral, determinar uma ordem de convergência quadrática na $L^{2}$-norma é um problema em aberto para os MVF com a decomposição Voronoï-Delaunay. Diversos esforços tem sido feitos para responder esta questão e limitam-se aos aspectos topológicos, por exemplo, Omnes (2011) dá uma resposta parcial e um aprimoramento importante em relação ao que conhece-se até agora.

Definidos os critérios necessários de estabilidade das soluções construídas pelo MVF na norma $H^{1}$, pudemos determinar as estimativas de erro na norma do máximo. Não é de nosso conhecimento que avanços nesse sentido tenham sido previamente descritos na literatura, em particular na esfera em decomposições Voronoï-Delaunay. Para tanto fizemos uso das funções de Green regularizadas para superfícies, definidas por Aubin (2013); Hebey (1996). Em seguida, definimos uma formulação variacional para as funções de Green com o objetivo de usar análises de convergência pontual dos MEF examinadas por Demlow (2009); Kröner (2017) e Kovács e Power Guerra (2018). Deste modo pudemos estabelecer uma estimativa fraca da estabilidade e obter estimativas da ordem de convergência nas max e $W^{1, \infty}$-normas para as decomposições Voronö̈-Delaunay não otimizadas (NOPT) e otimizadas sob o critério da SCVT. Os resultados obtidos nesse trabalho garantem convergência sub-linear (no caso NOPT) e super-linear (no caso SCVT). Não obstante, contém um fator logarítmico causado pelo uso das funções lineares na decomposição primal de Delaunay, que não pode ser evitado como examinado em domínios Euclidianos por Scott (1976) e Rannacher e Scott (1982).

\section{Organização do trabalho}

A análise das estimativas de erro para as discretizações do Laplaciano está organizada em quatro capítulos que visam sistematizar o conteúdo abordado nesta pesquisa. Cada capítulo possui uma breve introdução na qual contextualizamos seus objetivos. No Capítulo 1, fazemos uma rápida introdução das notações propriedades geométricas da esfera unitária $\mathbb{S}^{2}$, assim como de diversos resultados analíticos bem conhecidos da equação de Laplace em $\mathbb{S}^{2}$. Em particular, é definida a boa colocação (existência e unicidade) do problema e estabelecemos estimativas a priori da estabilidade e regularidade das soluções. O Capítulo 2 apresenta uma estrutura clássica da discretização de $\mathbb{S}^{2}$. 
Centra-se na construção recursiva das malhas esféricas geodésicas icosaédricas junto com duas das suas otimizações usuais na literatura, a saber a SCVT e a HR95.

Em seguida, no Capítulo 3, descrevemos um esquema de volumes finitos para o Laplaciano, reconhecido atualmente como um modelo numérico eficaz em relação à estrutura da geometria introduzida no capítulo anterior. Conforme a literatura, usamos uma forma variacional para esquema de volumes finitos conservativo no sentido dos MEFL, com o proposito de definir a boa colocação discreta do problema. Posteriormente, definimos a estabilidade das soluções aproximadas.

O Capítulo 4 é dedicado às estimativas de erro para o esquema de volumes finitos. A abordagem apresentada combina então os MVF com os MEFL para mostrar as principais contribuições desta tese. Inicialmente, estabelecemos ordens de convergência um nas normas $H^{1}$ e $L^{2}$ para a malha NOPT. Em seguida, obtemos as estimativas na max e $W^{1, \infty}$-norma usando funções de Green regularizadas como funções auxiliares para as malhas NOPT e SCVT. Por fim, apresentamos evidências numéricas, confirmando adequadamente as estimativas relatadas. Conclusões e comentários finais deste trabalho são dados no capítulo final.

O Apêndice A contem as provas da consistência fraca do MVF nas $L^{2}$ e max-normas. O Apêndice B reúne algumas considerações práticas sobre cálculo e ferramentas de geometria esférica. Por fim, descrevemos no Apêndice C considerações técnicas da implementação para resolver o sistema linear gerado a partir do Laplaciano discreto. 


\section{Capítulo 1}

\section{Preliminares}

O propósito deste primeiro capítulo será estabelecer parte da notação e a terminologia, bem como algumas ideias básicas de geometria esférica que são imprescindíveis para o estudo do operador discreto de Laplace na esfera unitária. Em primeiro lugar, apresentaremos uma breve revisão das propriedades topológicas do espaço Euclidiano $\mathbb{R}^{3}$ e sua importância quando consideramos a esfera como um dos seus subconjuntos. Em seguida, estabeleceremos algumas definições dos espaços de funções, em particular os espaços de Sobolev em $\mathbb{S}^{2}$, junto com alguns dos seus teoremas clássicos. Por fim, deduziremos a boa colocação do problema contínuo e daremos estimativas a priori de estabilidade e regularidade das soluções.

\subsection{Propriedades geométricas em $\mathbb{S}^{2}$}

Nesta seção, apresentaremos uma série de generalidades e noções que serão usadas nos Capítulos 2 e 3 . Consideremos o espaço Euclidiano $\mathbb{R}^{3}$, sejam $\mathrm{x}=\left(x_{1}, x_{2}, x_{3}\right)$ e $\mathrm{y}=\left(y_{1}, y_{2}, y_{3}\right)$ pontos no $\mathbb{R}^{3}$, então a norma Euclidiana e o produto escalar são definidos respectivamente como

$$
\|\mathrm{x}\|_{\mathbb{R}^{3}}=\left(\sum_{i=1}^{3} x_{i}^{2}\right)^{1 / 2}, \quad(\mathrm{x}, \mathrm{y})_{\mathbb{R}^{3}}=\sum_{i=1}^{3} x_{i} y_{i}
$$

As vezes usaremos a notação simplificada $\|\cdot\|$ para denotar $\|\cdot\|_{\mathbb{R}^{3}}$. Se $\Omega \subset \mathbb{R}^{3}$, denotaremos por $\operatorname{cl}(\Omega)$ o fecho de $\Omega$ em $\mathbb{R}^{3}$ e o interior $\operatorname{Int}(\Omega)$ de $\Omega$ em $\mathbb{R}^{3}$. O símbolo $\partial \Omega$ será considerado como a fronteira de $\Omega$, i.e., $\partial \Omega=\operatorname{cl}(\Omega) \cap\left(\mathbb{R}^{3} \backslash \Omega\right)$. Conforme Aubin (2013) e Hebey (1996), a esfera é uma variedade Riemanniana 2-dimensional compacta de $\mathbb{R}^{3}$. Apenas para o desenvolvimento deste trabalho, consideramos a esfera unitária centrada na origem do $\mathbb{R}^{3}$ definida por

$$
\mathbb{S}^{2}:=\left\{\mathrm{x} \in \mathbb{R}^{3}:\|\mathrm{x}\|_{\mathbb{R}^{3}}=1\right\}
$$

onde $\|\cdot\|_{\mathbb{R}^{3}}$ denota a norma Euclidiana no $\mathbb{R}^{3}$.

Apesar que o trabalho não tem um enfoque de geometria diferencial, vamos considerar diversas ideias do cálculo em superfícies, descritas nos textos de Morimoto (1998) e Atkinson e Han (2012). Dados dois pontos quaisquer $\mathrm{x}, \mathrm{y} \in \mathbb{S}^{2}$, a curva de menor comprimento (geodésica) contida em $\mathbb{S}^{2}$ ligando x e y é um arco de circunferência determinado pela interseção de $\mathbb{S}^{2}$ com o plano que passa por x, y e a origem de $\mathbb{S}^{2}$. A distância geodésica entre x e y é definida por

$$
\mathrm{d}(\mathrm{x}, \mathrm{y}):=\arccos \left((\mathrm{x}, \mathrm{y})_{\mathbb{R}^{3}}\right) \in[0, \pi], \quad \text { para cada } \mathrm{x}, \mathrm{y} \in \mathbb{S}^{2},
$$

onde $(\cdot, \cdot)_{\mathbb{R}^{3}}$ denota o produto escalar Euclidiano. Observe que, $\mathrm{d}(\cdot, \cdot)$ é uma distância adequada com a curvatura de $\mathbb{S}^{2}$ ao medir o ângulo formado pelos pontos e ademais, representa o caminho que possui a menor distância entre x e y (Aubin, 2013; Morimoto, 1998). De acordo com Atkinson e Han (2012, pp. 4), podemos definir uma equivalência entre as distâncias geodésica e Euclidiana, dada como

$$
\frac{2}{\pi} \mathrm{d}(\mathrm{x}, \mathrm{y}) \leq\|\mathrm{x}-\mathrm{y}\|_{\mathbb{R}^{3}} \leq \mathrm{d}(\mathrm{x}, \mathrm{y}), \quad \text { para cada } \mathrm{x}, \mathrm{y} \in \mathbb{S}^{2} .
$$

A desigualdade segue se escrevemos a distância Euclidiana por $\|\mathrm{x}-\mathrm{y}\|_{\mathbb{R}^{3}}=\sqrt{2\left(1-(\mathrm{x}, \mathrm{y})_{\mathbb{R}^{3}}\right)} \mathrm{e}$ 
usando a desigualdade $\frac{2}{\pi} t \leq \sin (t) \leq t$, para cada $t \in\left[0, \frac{\pi}{2}\right]$.

Dado um polígono esférico $\widetilde{\mathrm{V}} \subset \mathbb{S}^{2}$ delimitado por geodésicas. Denotaremos por $\operatorname{cl}(\widetilde{\mathrm{V}})$ o fecho de $\widetilde{V}$ e Int $(\widetilde{V})$ o interior de $\widetilde{V}$ em $\mathbb{S}^{2}$. Usaremos também o símbolo $\partial \widetilde{\mathrm{V}}$ para denotar a fronteira de $\widetilde{\mathrm{V}}$, i.e., $\partial \widetilde{\mathrm{V}}=\operatorname{cl}(\widetilde{\mathrm{V}}) \cap\left(\mathbb{S}^{2} \backslash \widetilde{\mathrm{V}}\right)$. Frequentemente serão usadas as medidas da área superficial $S(\widetilde{\mathrm{V}})$ para algum subconjunto $\widetilde{\mathrm{V}} \subset \mathbb{S}^{2}$ e do comprimento do arco geodésico $m(\Gamma)$ para alguma geodésica $\Gamma \subset \mathbb{S}^{2}$. Definimos então,

$$
S(\widetilde{\mathrm{V}})=\int_{\widetilde{\mathrm{V}}} d s(\mathrm{x}), \quad m(\Gamma)=\int_{\Gamma} d \gamma(\mathrm{x}),
$$

onde $d s(\mathrm{x})$ e $d \gamma(\mathrm{x})$ representam a medidas de Lebesgue na superfície da esfera $\mathbb{S}^{2}$ (Aubin, 2013; Morimoto, 1998).

\subsection{Espaços de funções}

Nesta seção, apresentaremos algumas noções fundamentais sobre os espaços de funções em $\mathbb{S}^{2}$ tomadas de Dziuk e Elliott (2013). Essas definições e resultados serão usados para descrever os ideias dos Capítulos 3 e 4 . Consideremos $u: \mathbb{S}^{2} \rightarrow \mathbb{R}$ uma função de valor real definida em $\mathbb{S}^{2}$ e denotamos por $C\left(\mathbb{S}^{2}\right)$ o espaço de Banach das funções contínuas com norma

$$
\|u\|_{C\left(\mathbb{S}^{2}\right)}:=\sup \left\{|u(\mathrm{x})|: \mathrm{x} \in \mathbb{S}^{2}\right\}, \quad \text { para cada } u \in C\left(\mathbb{S}^{2}\right) .
$$

Denotamos por $L^{2}\left(\mathbb{S}^{2}\right)$ o espaço de Hilbert de funções reais quadrado integráveis em $\mathbb{S}^{2}$ com produto escalar e norma induzida definidas respectivamente por

$$
(u, v):=\int_{\mathbb{S}^{2}} u(\mathrm{x}) v(\mathrm{x}) d s(\mathrm{x}), \quad \text { para cada } u, v \in L^{2}\left(\mathbb{S}^{2}\right), \quad\|u\|_{L^{2}\left(\mathbb{S}^{2}\right)}:=(u, u)^{1 / 2} .
$$

Note-se que, o espaço $C\left(\mathbb{S}^{2}\right)$ com o produto escalar em $L^{2}\left(\mathbb{S}^{2}\right)$ não é Banach, logo o fecho de $C\left(\mathbb{S}^{2}\right)$ em relação à $L^{2}$-norma, é $L^{2}\left(\mathbb{S}^{2}\right)$, i.e., se $u \in L^{2}\left(\mathbb{S}^{2}\right)$, existe uma sequência $\left\{u_{n}\right\}_{n \in \mathbb{N}} \subset C\left(\mathbb{S}^{2}\right)$ tal que

$$
\left\|u_{n}-u\right\|_{L^{2}\left(\mathbb{S}^{2}\right)} \longrightarrow 0, \quad \text { quando } n \longrightarrow \infty .
$$

Para cada $u \in C\left(\mathbb{S}^{2}\right)$ definiremos o seu módulo de continuidade,

$$
m(u ; \delta):=\sup \left\{|u(\mathrm{x})-u(\mathrm{y})|: \mathrm{x}, \mathrm{y} \in \mathbb{S}^{2},\|\mathrm{x}-\mathrm{y}\|_{\mathbb{R}^{3}}<\delta\right\},
$$

para $\delta \in(0,1)$. Então

$$
m(u ; \delta) \longrightarrow 0, \quad \text { quando } \delta \longrightarrow 0 .
$$

Para $\delta \in(0,1)$ definimos o conjunto

$$
\Omega_{\delta}:=\left\{\mathrm{x}^{*} \in \mathbb{R}^{3} \backslash\{0\}: 1-\delta \leq\left\|\mathrm{x}^{*}\right\|_{\mathbb{R}^{3}} \leq 1+\delta\right\},
$$

tal que $\mathbb{S}^{2} \subset \Omega_{\delta}$ para cada $\delta \in(0,1)$. Em vista disso, para a análise de convergência em $\mathbb{S}^{2}$ é usual considerar a extensão $u^{\Omega}$ da função $u$ ao conjunto $\Omega_{\delta}$, então dado $\mathrm{x}=\frac{\mathrm{x}^{*}}{\left\|\mathrm{x}^{*}\right\|} \in \mathbb{S}^{2}$, segue

$$
u^{\Omega}\left(\mathrm{x}^{*}\right):=u\left(\frac{\mathrm{x}^{*}}{\left\|\mathrm{x}^{*}\right\|}\right)=u(\mathrm{x}), \quad \text { para } 0 \neq \mathrm{x}^{*} \in \Omega_{\delta} .
$$

Observe-se que $u^{\Omega}$ se estende naturalmente por meio da relação acima a uma função definida em $\mathbb{R}^{3} \backslash\{0\}$. Ademais, se $u^{\Omega}: \Omega_{\delta} \rightarrow \mathbb{R}$, denotamos por $C\left(\Omega_{\delta}\right)$ o espaço de funções contínuas em $\Omega_{\delta}$ com norma,

$$
\left\|u^{\Omega}\right\|_{C\left(\Omega_{\delta}\right)}:=\sup \left\{\left|u^{\Omega}\left(\mathrm{x}^{*}\right)\right|: \mathrm{x}^{*} \in \Omega_{\delta}\right\}, \quad \text { para cada } u^{\Omega} \in C\left(\Omega_{\delta}\right) .
$$

Examinaremos agora o fundamento de diferenciabilidade o qual é bastante natural em $\mathbb{S}^{2}$, salvo alguns cuidados, pois certos procedimentos de cálculo elementar em $\mathbb{R}^{3}$ podem ser falsos em $\mathbb{S}^{2}$. Vamos introduzir a definição de gradiente tangencial a $\mathbb{S}^{2}$ para alguma função diferenciável $u: \mathbb{S}^{2} \rightarrow \mathbb{R}$, segundo a seguinte interpretação: definamos $\overrightarrow{\mathrm{v}}$ um vetor no ponto $\mathrm{x} \in \mathbb{S}^{2}$, o vetor $\overrightarrow{\mathrm{v}}$ 
pode ser decomposto unicamente como soma de dois vetores, o primeiro será tangente a $\mathbb{S}^{2}$ em $\mathrm{x}$, conhecido como a componente tangencial e o segundo será perpendicular a $\mathbb{S}^{2} \mathrm{em} \mathrm{x}$ conhecido como a componente normal. Assim, surge a expressão

$$
\overrightarrow{\mathrm{v}}=\overrightarrow{\mathrm{v}}_{T}+\overrightarrow{\mathrm{v}}_{\perp}
$$

onde $\overrightarrow{\mathrm{v}}_{T}$ denota a componente tangencial e $\overrightarrow{\mathrm{v}}_{\perp}$ a componente normal. Os termos $\overrightarrow{\mathrm{v}}_{T}$ e $\overrightarrow{\mathrm{v}}_{\perp}$ são ortogonais entre si. Agora, se na definição anterior, substituímos $\overrightarrow{\mathrm{v}}$ por $\nabla u$, o gradiente usual no $\mathbb{R}^{3}$ da função $u$, com $\nabla=\left(\partial_{1}, \partial_{2}, \partial_{3}\right)$. Podemos calcular a componente tangencial (tangente à superfície esférica no ponto $\mathrm{x}$ ), definindo a componente normal como

$$
(\nabla u(\mathrm{x}))_{\perp}:=\left(\nabla u(\mathrm{x}) \cdot \overrightarrow{\mathrm{n}}_{\mathbb{S}^{2}, \mathrm{x}}\right) \overrightarrow{\mathrm{n}}_{\mathbb{S}^{2}, \mathrm{x}},
$$

onde "." denota o produto escalar e $\overrightarrow{\mathrm{n}}_{\mathbb{S}^{2}, \mathrm{x}}$ é o vetor normal unitário a $\mathbb{S}^{2}$ no ponto x. Consequentemente, a componente tangencial é dada como

$$
(\nabla u(\mathrm{x}))_{T}=\nabla u(\mathrm{x})-\left(\nabla u(\mathrm{x}) \cdot \overrightarrow{\mathrm{n}}_{\mathbb{S}^{2}, \mathrm{x}}\right) \overrightarrow{\mathrm{n}}_{\mathbb{S}^{2}, \mathrm{x}} \cdot
$$

Em outras palavras, a componente tangencial $(\nabla u(\mathrm{x}))_{T}$ da função $u$ é o gradiente usual menos a componente normal à esfera $\mathbb{S}^{2}$ no ponto $\mathrm{x}$. Observe ademais, que todos as componentes tangenciais a uma curva em $\mathbb{S}^{2}$ no ponto $\mathrm{x} \in \mathbb{S}^{2}$ formam um plano tangente $\mathbb{T}_{\mathbb{S}^{2}, \mathrm{x}}$, e um vetor perpendicular a $\mathbb{T}_{\mathbb{S}^{2}, \mathrm{x}}$ é o vetor normal $\overrightarrow{\mathrm{n}}_{\mathbb{S}^{2}, \mathrm{x}}$ no ponto x. Para simplificar a notação, denotaremos a componente tangencial $(\nabla u(\mathrm{x}))_{T}$ por $\nabla_{s} u(\mathrm{x})$, para cada $\mathrm{x} \in \mathbb{S}^{2}$. Desta maneira, geramos a seguinte definição.

Definição 1.1 (Gradiente tangencial). Sejam $\mathbb{S}^{2} \subset \mathbb{R}^{3}$ e u $\mathbb{S}^{2} \rightarrow \mathbb{R}$ uma função diferenciável em $\mathrm{x} \in \mathbb{S}^{2}$. Definimos o gradiente tangencial de $u$ em $\mathrm{x} \in \mathbb{S}^{2}$ por

$$
\nabla_{s} u(\mathrm{x}):=\left(\partial_{s, 1}, \partial_{s, 2}, \partial_{s, 3}\right) u(\mathrm{x})=\nabla u^{\Omega}(\mathrm{x})-\left(\nabla u^{\Omega}(\mathrm{x}) \cdot \overrightarrow{\mathrm{n}}_{\mathbb{S}^{2}, \mathrm{x}}\right) \overrightarrow{\mathrm{n}}_{\mathbb{S}^{2}, \mathrm{x}},
$$

onde $\partial_{s, n}$ denota a derivada parcial tangencial na coordenada $x_{n}$, do ponto $\mathrm{x}, \overrightarrow{\mathrm{n}}_{\mathbb{S}^{2}, \mathrm{x}}$ é o vetor normal a $\mathbb{S}^{2}$ em $\mathrm{x}$. Aqui $u^{\Omega}$ é a extensão suave de $u$ a $\Omega_{\delta} \subset \mathbb{R}^{3}$, tal que $\left.u^{\Omega}\right|_{\mathbb{S}^{2}}=u$. Em particular, $u^{\Omega}(\mathrm{x})=u(\mathrm{x})$ para cada $\mathrm{x} \in \mathbb{S}^{2}$.

De forma análoga, pode-se construir o operador de Laplace em $\mathbb{S}^{2}$ :

Definição 1.2 (Operador de Laplace-Beltrami). Sejam $\mathbb{S}^{2} \subset \mathbb{R}^{3}$ e u: $\mathbb{S}^{2} \rightarrow \mathbb{R}$ uma função duas vezes continuamente diferenciável. O operador de Laplace-Beltrami da função u é dado como

$$
\Delta_{s} u(\mathrm{x}):=\sum_{n=1}^{3} \partial_{s, n} \partial_{s, n} u(\mathrm{x})=\nabla_{s} \cdot \nabla_{s} u(\mathrm{x})=\nabla \cdot \nabla_{s} u(\mathrm{x})-\overrightarrow{\mathrm{n}}_{\mathbb{S}^{2}, \mathrm{x}}\left[\nabla\left(\nabla_{s} u(\mathrm{x})\right)\right] \overrightarrow{\mathrm{n}}_{\mathbb{S}^{2}, \mathrm{x}}^{t},
$$

onde $\overrightarrow{\mathrm{n}}_{\mathbb{S}^{2}, \mathrm{x}}^{t}$ denota o vetor transposto de $\overrightarrow{\mathrm{n}}_{\mathbb{S}^{2}, \mathrm{x}}$ e $\nabla\left(\nabla_{s} u(\mathrm{x})\right)$ representa a matriz que contem todas as derivadas de $\nabla_{s} u$.

Caso seja necessário para a diferenciação de ordem maior de funções em $\Omega_{\delta} \subset \mathbb{R}^{3}$ ou $\mathbb{S}^{2}$ usamos a notação de multi-índice. Um multi-índice é o vetor $\alpha=\left(\alpha_{1}, \alpha_{2}, \alpha_{3}\right)$, para cada $0 \leq \alpha_{i} \in \mathbb{N}$, com comprimento dado por $|\alpha|=\sum_{i=1}^{3} \alpha_{i}$. Denotamos por $\nabla^{\alpha}$ o operador diferencial de ordem $|\alpha|$ como

$$
\nabla^{\alpha}=\partial_{1}^{\alpha_{1}} \partial_{2}^{\alpha_{2}} \partial_{3}^{\alpha_{3}}
$$

onde $\partial_{n}(n \in\{1,2,3\})$ denota a derivada parcial usual no $\mathbb{R}^{3}$ da $n$-ésima coordenada do ponto $\mathrm{x}=\left(x_{1}, x_{2}, x_{3}\right)$. De forma análoga, definimos $\nabla_{s}^{\alpha}$ o operador diferencial tangencial de ordem $|\alpha|$ como

$$
\nabla_{s}^{\alpha}=\partial_{s, 1}^{\alpha_{1}} \partial_{s, 2}^{\alpha_{2}} \partial_{s, 3}^{\alpha_{3}}
$$

Para $k \geq 0$ e $\alpha$ um multi-índice, denotaremos por $C^{k}\left(\Omega_{\delta}\right)$ o espaço de funções contínuas $u^{\Omega}$ junto 
com todas as suas derivadas $\nabla^{\alpha} u^{\Omega}$ contínuas de ordem $|\alpha| \leq k$ em $\Omega_{\delta} \subset \mathbb{R}^{3}$, i.e.,

$$
C^{k}\left(\Omega_{\delta}\right):=\left\{u: \Omega_{\delta} \rightarrow \mathbb{R}: \nabla^{\alpha} u \in C\left(\Omega_{\delta}\right) \quad \operatorname{com}|\alpha| \leq k\right\} .
$$

O espaço $C^{k}\left(\Omega_{\delta}\right)$ é Banach com norma,

$$
\left\|u^{\Omega}\right\|_{C^{k}\left(\Omega_{\delta}\right)}:=\sup _{0 \leq|\alpha| \leq k}\left\{\left|\nabla^{\alpha} u^{\Omega}\left(\mathrm{x}^{*}\right)\right|: \mathrm{x}^{*} \in \Omega_{\delta}\right\} .
$$

Uma função $u$ é dita $k$-vezes continuamente diferenciável em $\mathbb{S}^{2}$ sempre que $u^{\Omega}$ seja $k$-vezes continuamente diferenciável em $\Omega_{\delta}$. Destaca-se que a diferenciabilidade da função $u$ é independente da escolha do $\delta \in(0,1)$ (Dziuk e Elliott, 2013; Morimoto, 1998). Então, para $k \geq 0$, seja $C^{k}\left(\mathbb{S}^{2}\right.$ ) o espaço vetorial que consiste de todas as funções contínuas $u$ junto com todas as suas derivadas tangenciais $\nabla_{s}^{\alpha} u$ contínuas de ordem $|\alpha| \leq k$ em $\mathbb{S}^{2}$. O espaço $C^{k}\left(\mathbb{S}^{2}\right)$ é um espaço de Banach com norma

$$
\|u\|_{C^{k}\left(\mathbb{S}^{2}\right)}:=\left\|u^{\Omega}\right\|_{C^{k}\left(\Omega_{\delta}\right)} \quad \text { para cada } u \in C^{k}\left(\mathbb{S}^{2}\right) .
$$

Em particular, temos que $C^{\infty}\left(\mathbb{S}^{2}\right)=\bigcap_{k=0}^{\infty} C^{k}\left(\mathbb{S}^{2}\right)$ é o espaço linear das funções contínuas infinitamente diferenciáveis em $\mathbb{S}^{2}$.

\subsection{Espaços de Sobolev em $\mathbb{S}^{2}$}

Sabemos que no desenvolvimento da análise das soluções das EDPs tanto analíticas como numéricas, é indispensável o uso de certos espaços funcionais, em particular, os espaços de Sobolev. Fazemos uma pequena apresentação sobre generalidades e resultados em relação a esses espaços. As noções em principio, estão lideradas pela formulação variacional aliada com a fórmula de Green. Ao leitor interessado em profundar nos detalhes destes temas para superfícies em geral recomendamos a consulta dos textos de: Hebey (1996) e Aubin (2013).

Como de costume, se $p$ é algum número entre $1 \leq p \leq \infty$, denotamos por $L^{p}\left(\mathbb{S}^{2}\right)$ os espaços de funções mensuráveis $u: \mathbb{S}^{2} \rightarrow \mathbb{R}$ em relação à medida de superfície $d s$, tal que

$$
\begin{aligned}
\int_{\mathbb{S}^{2}}|u(\mathrm{x})|^{p} d s(\mathrm{x})<\infty, & 1 \leq p<\infty, \\
\operatorname{ess} \sup _{\mathrm{x} \in \mathbb{S}^{2}}|u(\mathrm{x})|<\infty, & p=\infty
\end{aligned}
$$

onde $\operatorname{ess} \sup (u(\mathrm{x}))=\inf \{C \geq 0:|u(\mathrm{x})| \leq C, \quad$ quase sempre $\}$, com normas

$$
\begin{aligned}
\|u\|_{L^{p}\left(\mathbb{S}^{2}\right)} & =\left(\int_{\mathbb{S}^{2}}|u(\mathrm{x})|^{p} d s(\mathrm{x})\right)^{1 / p}, \quad 1 \leq p<\infty, \\
\|u\|_{L^{\infty}\left(\mathbb{S}^{2}\right)} & =\operatorname{ess}_{\sup _{\mathrm{x} \in \mathbb{S}^{2}}|u(\mathrm{x})|, \quad p=\infty .}
\end{aligned}
$$

Os espaços $L^{p}\left(\mathbb{S}^{2}\right)$ com $1 \leq p \leq \infty$ são espaços de Banach. Note-se, que os espaços $C\left(\mathbb{S}^{2}\right)$ e $C^{1}\left(\mathbb{S}^{2}\right)$ são densos em $L^{p}\left(\mathbb{S}^{2}\right)$ para $1 \leq p<\infty$. Utilizamos essa notação para a seguinte definição.

Definição 1.3 (Hebey, 1996). Sejam $k$ um inteiro não negativo e $1 \leq p \leq \infty$. O espaço de Sobolev $W^{k, p}\left(\mathbb{S}^{2}\right)$ é o conjunto de todas as funções $u \in L^{p}\left(\mathbb{S}^{2}\right)$ tal que para cada multi-índice $\alpha$, com $|\alpha| \leq k$, a $\alpha$-ésima derivada tangencial fraca $\nabla_{s}^{\alpha} u$ existe e pertence ao espaço $L^{p}\left(\mathbb{S}^{2}\right)$, i.e.,

$$
W^{k, p}\left(\mathbb{S}^{2}\right):=\left\{u \in L^{p}\left(\mathbb{S}^{2}\right): \nabla_{s}^{\alpha} u \in L^{p}\left(\mathbb{S}^{2}\right), \quad \text { para cada } 0 \leq|\alpha| \leq k\right\} .
$$

Definimos agora a norma em $W^{k, p}\left(\mathbb{S}^{2}\right)$ por

$$
\begin{aligned}
\|u\|_{W^{k, p}\left(\mathbb{S}^{2}\right)} & =\left(\sum_{0 \leq|\alpha| \leq k}\left\|\nabla_{s}^{\alpha} u\right\|_{L^{p}\left(\mathbb{S}^{2}\right)}^{p}\right)^{1 / p}, \quad 1 \leq p<\infty, \\
\|u\|_{W^{k, \infty}\left(\mathbb{S}^{2}\right)} & =\max _{0 \leq|\alpha| \leq k}\left\|\nabla_{s}^{\alpha} u\right\|_{L^{\infty}\left(\mathbb{S}^{2}\right)}, \quad p=\infty
\end{aligned}
$$


Resultados sobre os espaços $W^{k, p}\left(\mathbb{S}^{2}\right)$ ser Banach ou Hilbert estão contidos nos textos de Hebey (1996) e Aubin (2013).

Regularmente empregaremos $p=2$ para simplificar $W^{k, 2}\left(\mathbb{S}^{2}\right)=H^{k}\left(\mathbb{S}^{2}\right)$, que são espaços de Hilbert das funções quadrado integráveis com derivadas tangenciais fracas de ordem menor ou igual que $k$. Note ademais que se $k=0$ obtemos o espaço usual $W^{0,2}\left(\mathbb{S}^{2}\right)=L^{2}\left(\mathbb{S}^{2}\right)$. Em particular, para $k=2$ e $p=2$ temos para alguma função $u \in H^{2}\left(\mathbb{S}^{2}\right)$, que

$$
\|u\|_{H^{2}\left(\mathbb{S}^{2}\right)}=\left(\|u\|_{L^{2}\left(\mathbb{S}^{2}\right)}^{2}+\left\|\nabla_{s} u\right\|_{L^{2}\left(\mathbb{S}^{2}\right)}^{2}+\sum_{|\alpha|=2}\left\|\nabla_{s}^{\alpha} u\right\|_{L^{2}\left(\mathbb{S}^{2}\right)}^{2}\right)^{1 / 2} .
$$

\subsubsection{Espaço quociente}

Convém notar que o trabalho centra-se em um conjunto especial de funções a saber, vamos trabalhar naqueles subespaços de funções que são ortogonais a constantes em $L^{2}\left(\mathbb{S}^{2}\right)$. Escolhemos um subespaço fechado do espaço $H^{1}\left(\mathbb{S}^{2}\right)$, que consiste de todas as funções $v \in H^{1}\left(\mathbb{S}^{2}\right)$ satisfazem a condição

$$
(v, 1)=\int_{\mathbb{S}^{2}} v(\mathrm{x}) d s(\mathrm{x})=0 .
$$

Definimos o espaço quociente equipado com a $H^{1}$-norma por

$$
H_{Q}^{1}\left(\mathbb{S}^{2}\right):=H^{1}\left(\mathbb{S}^{2}\right) / \mathbb{R}=\left\{v \in H^{1}\left(\mathbb{S}^{2}\right): \int_{\mathbb{S}^{2}} v(\mathrm{x}) d s(\mathrm{x})=0\right\} .
$$

O seguinte resultado será útil na hora de provar a coercividade do operador de Laplace e sua prova está no texto de Hebey (1996, Lema 3.8, pp. 24).

Proposição 1.1 (Desigualdade de Poincaré-Friedrich). Existe uma constante $C_{P} \in \mathbb{R}_{+}$, tal que para cada $u \in H_{Q}^{1}\left(\mathbb{S}^{2}\right)$ vale a desigualdade,

$$
\left(\int_{\mathbb{S}^{2}}|u(\mathrm{x})|^{2} d s(\mathrm{x})\right)^{1 / 2} \leq C_{P}\left(\int_{\mathbb{S}^{2}}\left|\nabla_{s} u(\mathrm{x})\right|^{2} d s(\mathrm{x})\right)^{1 / 2} .
$$

\subsection{O modelo contínuo}

Nesta seção, vamos descrever o problema contínuo para o desenvolvimento da análise numérica dos Capítulos 3 e 4. Sejam $\mathbb{S}^{2}=\left\{\mathrm{x} \in \mathbb{R}^{3}:\|\mathrm{x}\|=1\right\}$ a esfera unitária e a função $f \in L^{2}\left(\mathbb{S}^{2}\right)$ que satisfaz a condição de compatibilidade

$$
(f, 1)=\int_{\mathbb{S}^{2}} f(\mathrm{x}) d s(\mathrm{x})=0
$$

onde $d s(\mathrm{x})$ denota é a medida de superfície. Seja também a função $u \in H^{2}\left(\mathbb{S}^{2}\right)$ que resolve o problema

$$
-\Delta_{s} u(\mathrm{x})=f(\mathrm{x}), \quad \text { para cada } \mathrm{x} \in \mathbb{S}^{2},
$$

onde $-\Delta_{s}$ representa o operador de Laplace em $\mathbb{S}^{2}$. Visto que $\partial \mathbb{S}^{2}=\emptyset$, não são necessárias condições de fronteira. Enunciamos as duas questões fundamentais que se colocam no estudo de (1.4):

(Q.1) Existência: a equação (1.4) possui solução se $f$ satisfaz a condição de compatibilidade (1.3).

(Q.2) Unicidade: a solução pode exibir não unicidade, já que o operador de Laplace na esfera possui Kernel não-trivial, i.e., qualquer constante $C \in \mathbb{R}$ é também solução da equação homogênea,

$$
-\Delta_{s}(u(\mathrm{x})+C)=0, \quad \text { para cada } \mathrm{x} \in \mathbb{S}^{2} .
$$

Então, para garantir a unicidade, requeremos que a solução $u$ pertença ao espaço $H^{2}\left(\mathbb{S}^{2}\right) \cap$ $H_{Q}^{1}\left(\mathbb{S}^{2}\right)$, cujos elementos são funções duas vezes diferenciáveis ortogonais a constantes no 
espaço $L^{2}\left(\mathbb{S}^{2}\right)$.

No estudo, se (Q.1) e (Q.2) são satisfeitas, diremos que o problema (1.4) está bem posto ou que possui boa colocação contínua.

\subsubsection{Uma formulação variacional}

Podemos definir uma forma variacional da equação (1.4) no espaço $H_{Q}^{1}\left(\mathbb{S}^{2}\right)$. Para quaisquer $u, v \in H_{Q}^{1}\left(\mathbb{S}^{2}\right)$ definimos o funcional bilinear $\mathcal{A}(\cdot, \cdot)$ tal que

$$
\mathcal{A}(u, v)=\int_{\mathbb{S}^{2}} \nabla_{s} u(\mathrm{x}) \cdot \nabla_{s} v(\mathrm{x}) d s(\mathrm{x}) .
$$

A forma bilinear acima está bem definida no espaço $H_{Q}^{1}\left(\mathbb{S}^{2}\right) \times H_{Q}^{1}\left(\mathbb{S}^{2}\right)$.

Proposição 1.2 (Continuidade e coercividade). Seja $\mathcal{A}: H_{Q}^{1}\left(\mathbb{S}^{2}\right) \times H_{Q}^{1}\left(\mathbb{S}^{2}\right) \rightarrow \mathbb{R}$ o funcional bilinear. Então, existem constantes $C_{n} \in \mathbb{R}_{+}, n \in\{0,1\}$ tal que,

$$
\begin{aligned}
|\mathcal{A}(u, v)| & \leq C_{0}\|u\|_{H^{1}\left(\mathbb{S}^{2}\right)}\|v\|_{H^{1}\left(\mathbb{S}^{2}\right)}, \quad \text { para cada } u, v \in H_{Q}^{1}\left(\mathbb{S}^{2}\right), \\
\mathcal{A}(v, v) & \geq C_{1}\|v\|_{H^{1}\left(\mathbb{S}^{2}\right)}^{2}, \quad \text { para cada } v \in H_{Q}^{1}\left(\mathbb{S}^{2}\right) .
\end{aligned}
$$

Demonstração. Dado o funcional bilinear e as desigualdade de Cauchy-Schwarz e PoincaréFriedrich, temos para $u, v \in H_{Q}^{1}\left(\mathbb{S}^{2}\right)$ que

$$
\begin{aligned}
|\mathcal{A}(u, v)|=\left|\int_{\mathbb{S}^{2}} \nabla_{s} u(\mathrm{x}) \cdot \nabla_{s} v(\mathrm{x}) d s(\mathrm{x})\right| & \leq\left(\int_{\mathbb{S}^{2}}\left|\nabla_{s} u(\mathrm{x})\right|^{2} d s(\mathrm{x})\right)^{1 / 2}\left(\int_{\mathbb{S}^{2}}\left|\nabla_{s} v(\mathrm{x})\right|^{2} d s(\mathrm{x})\right)^{1 / 2} \\
& \leq\left\|\nabla_{s} u\right\|_{L^{2}\left(\mathbb{S}^{2}\right)}\left\|\nabla_{s} v\right\|_{L^{2}\left(\mathbb{S}^{2}\right)} \leq C_{0}\|u\|_{H^{1}\left(\mathbb{S}^{2}\right)}\|v\|_{H^{1}\left(\mathbb{S}^{2}\right)}
\end{aligned}
$$

em consequência segue (1.6). Considere $v \in H_{Q}^{1}\left(\mathbb{S}^{2}\right)$, assim

$$
\mathcal{A}(v, v)=\int_{\mathbb{S}^{2}} \nabla_{s} v(\mathrm{x}) \cdot \nabla_{s} v(\mathrm{x}) d s(\mathrm{x})=\int_{\mathbb{S}^{2}}\left|\nabla_{s} v(\mathrm{x})\right|^{2} d s(\mathrm{x})=\left\|\nabla_{s} v\right\|_{L^{2}\left(\mathbb{S}^{2}\right)}^{2} .
$$

Da desigualdade de Poincaré-Friedrich (Proposição 1.1), existe uma constante $C_{P} \in \mathbb{R}_{+}$, que independe de $v$, tal que

$$
\|v\|_{L^{2}\left(\mathbb{S}^{2}\right)}^{2} \leq C_{P}\left\|\nabla_{s} v\right\|_{L^{2}\left(\mathbb{S}^{2}\right)}^{2}
$$

e junto à equivalência nas normas, existe uma constante $C_{2} \in \mathbb{R}_{+}$tal que

$$
\|v\|_{H^{1}\left(\mathbb{S}^{2}\right)}^{2} \leq C_{2}\left\|\nabla_{s} v\right\|_{L^{2}\left(\mathbb{S}^{2}\right)}^{2} .
$$

Portanto,

$$
\mathcal{A}(v, v)=\left\|\nabla_{s} v\right\|_{L^{2}\left(\mathbb{S}^{2}\right)}^{2} \geq C_{1}\|v\|_{H^{1}\left(\mathbb{S}^{2}\right)}^{2} .
$$

para $C_{1}=1 / C_{2}$. Isto encerra a demostração.

Temos imposto a condição (1.3) para a função $f \in L^{2}\left(\mathbb{S}^{2}\right)$ no lado direito da equação de Laplace, logo o problema variacional de (1.4) é definido da seguinte forma: a única solução fraca $u \in H_{Q}^{1}\left(\mathbb{S}^{2}\right)$ de (1.4) satisfaz o problema,

$$
\mathcal{A}(u, v)=(f, v), \quad \text { para cada } v \in H_{Q}^{1}\left(\mathbb{S}^{2}\right) .
$$

De posse das informações precedentes, temos o resultado a seguir.

Teorema 1.1 (Unicidade da solução fraca). Seja $\mathbb{S}^{2}$ a esfera unitária e assuma que $f \in L^{2}\left(\mathbb{S}^{2}\right)$ satisfaz (1.3). Então, existe uma única solução $u \in H_{Q}^{1}\left(\mathbb{S}^{2}\right)$ do problema (1.8).

A demostração é uma aplicação do Teorema de Lax-Milgram (vide Brezis, 2010, Corolário 5.8, pp. 140), i.e., como consequência imediata da Proposição 1.2. A existência da solução fraca é um 
ingrediente importante nos requerimentos das estimativas de convergência dos esquemas numéricos, particularmente da estabilidade. Uma primeira estimativa a priori na $H^{1}$-norma é definida como segue:

Proposição 1.3 (Estabilidade). Seja $f \in L^{2}\left(\mathbb{S}^{2}\right)$ que satisfaz (1.3). Então, o problema variacional (1.8) tem única solução fraca $u \in H_{Q}^{1}\left(\mathbb{S}^{2}\right)$ e satisfaz a estimativa,

$$
\|u\|_{H^{1}\left(\mathbb{S}^{2}\right)} \leq C\|f\|_{L^{2}\left(\mathbb{S}^{2}\right)},
$$

para alguma constante $C \in \mathbb{R}_{+}$.

Demonstração. A prova é obtida direitamente da Proposição 1.2, combinando as desigualdade de Cauchy-Schwarz de Poincaré-Friedrich,

$$
\|u\|_{H^{1}\left(\mathbb{S}^{2}\right)}^{2} \leq C|\mathcal{A}(u, u)| \leq C|(f, u)| \leq C\|f\|_{L^{2}\left(\mathbb{S}^{2}\right)}\|u\|_{L^{2}\left(\mathbb{S}^{2}\right)} \leq C\|f\|_{L^{2}\left(\mathbb{S}^{2}\right)}\|u\|_{H^{1}\left(\mathbb{S}^{2}\right)} .
$$

Portanto, segue o resultado.

Provas similares podem ser consultadas no trabalhos de Herrmann et al. (2018), Demlow (2009) ou Dziuk e Elliott (2013). Por último, se $u \in H^{2}\left(\mathbb{S}^{2}\right) \cap H_{Q}^{1}\left(\mathbb{S}^{2}\right)$ é a única solução fraca de (1.8), podese estabelecer uma outra estimativa a priori na $H^{2}$-norma, i.e., uma estimativa de regularidade da solução.

Proposição 1.4 (Regularidade). Assuma $f \in L^{2}\left(\mathbb{S}^{2}\right)$ que satisfaz a condição de compatibilidade (1.3). Então, se a única solução fraca do problema (1.8) é tal que $u \in H^{2}\left(\mathbb{S}^{2}\right) \cap H_{Q}^{1}\left(\mathbb{S}^{2}\right)$, esta satisfaz a estimativa de regularidade

$$
\|u\|_{H^{2}\left(\mathbb{S}^{2}\right)} \leq C\|f\|_{L^{2}\left(\mathbb{S}^{2}\right)} .
$$

Demonstração. A prova segue diretamente usando a fórmula de Green para $u \in H^{2}\left(\mathbb{S}^{2}\right) \cap H_{Q}^{1}\left(\mathbb{S}^{2}\right)$ na equação (1.4). Em seguida, usar a estimativa definida na Proposição 1.3.

Com o anterior, temos definido o problema contínuo na sua forma variacional; especificamente, temos estabelecido os argumentos padrão da estabilidade e a regularidade da solução exata para o problema de Laplace em $\mathbb{S}^{2}$. O passo seguinte, será discretizar a esfera unitária e usa-la como domínio computacional para nosso problema discreto. 
PRELIMINARES 


\section{Capítulo 2}

\section{Malhas esféricas geodésicas icosaédricas}

Este capítulo tem como objetivo apresentar uma construção usual das malhas esféricas geodésicas icosaédricas em $\mathbb{S}^{2}$, usadas frequentemente na literatura na discretização de problemas da dinâmica de fluidos geofísicos (Heinze e Hense, 2002; Putman e Lin, 2007; Williamson, 2007). Nesse sentido, as malhas serão definidas recursivamente satisfazendo o princípio de dualidade VoronoïDelaunay. Descreveremos também duas das otimizações mais usadas na literatura em relação à qualidade de malha, a saber, a SCVT e HR95.

\subsection{Estruturas de malhas na esfera}

Historicamente foram usadas discretizações latitude-longitude, denominadas malhas geográficas, nos primeiros modelos globais de previsão do tempo. Esse tipo de discretizações é a forma mais simples de estabelecer um sistema de coordenadas para a superfície esférica e consiste na Figura 2.1a, por coordenadas esféricas, de um conjunto de segmentos de retas no plano de coordenadas angulares, com propriedades importantes como ortogonalidade e simetria.

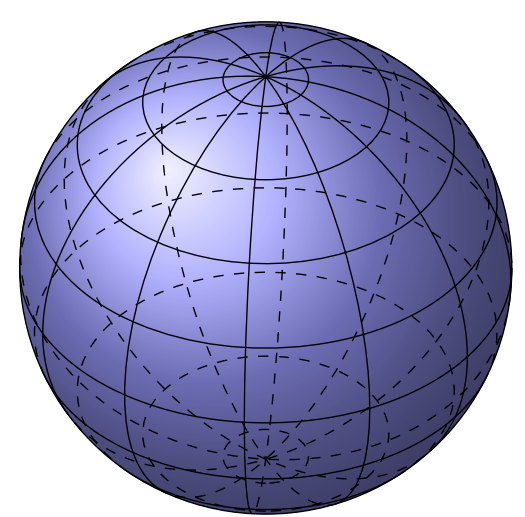

(a) Geográfica

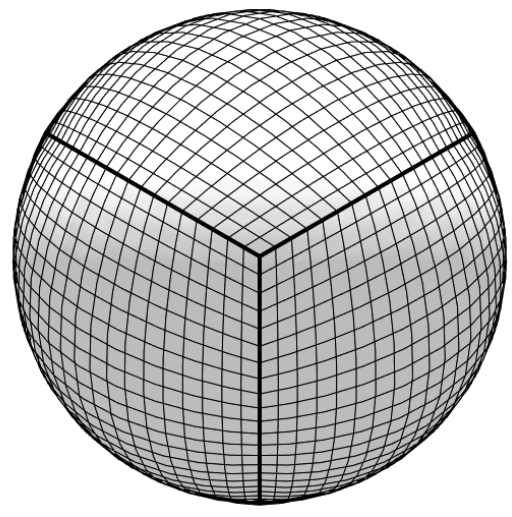

(b) Esfera cubada

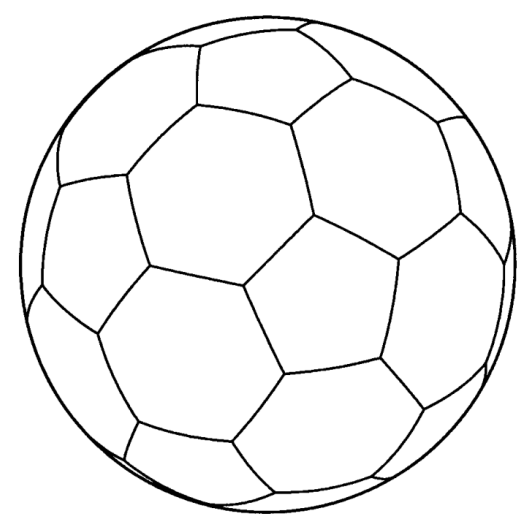

(c) Bucky ball

Figura 2.1: Estruturas de malhas: (a) a malha geográfica latitude-longitude. Imagem gerada no site: github, jobovy-stellarkinematics., (b) a esfera cubada baseada nas transformações dos seis lados de um hexaedro circunscrito na esfera $\mathbb{S}^{2}$. Imagem gerada no site: www.gfdl.noaa.gov do GFDL (Geophysical Fluid Dynamics Laboratory) da NOAA (National Oceanic and Atmospheric Administration) e (c) a malha Bucky-ball. Fonte: Heinze e Hense (2002).

A latitude (denotada por $\phi$ ) determina a posição de um ponto no hemisfério norte ou sul, i.e., a distância angular, medida em radianos, que varia desde 0 no equador até $\pi / 2$ nos polos (norte ou sul). Usa-se latitude positiva no hemisfério norte e negativa no hemisfério sul. Os círculos paralelos ao equador possuem latitude constante. A longitude (denotada $\operatorname{com} \theta$ ) é a distância angular, medida no plano do equador, entre o meridiano do ponto na superfície e o meridiano de Greenwich, que por convenção é considerado a longitude base ou nula na literatura. Neste caso, usa-se longitude negativa a oeste, e positiva a leste, variando assim entre $-\pi$ e $\pi$.

No entanto, este tipo de malhas possui uma concentração dos pontos próximos aos polos, logo o tamanho das células computacionais e a distância entre dois pontos adjacentes da malha 
dependem da latitude, gerando então uma malha anisotrópica. A convergência dos meridianos que se aproximam aos polos faz que o intervalo das distâncias longitudinais (medidas em distância em vez de graus) tenda para zero nos polos, logo o sistema de coordenadas torna-se singular perto dos polos. Essa convergência nos meridianos e a singularidade é conhecido como o problema do polo e foi vagamente definido no começo como uma dificuldade de custo computacional mais do que um problema fundamental (Murray, 1996; Williamson, 2007).

Diversas modificações foram sugeridas, por exemplo a proposta de Randall et al. (1998), apresentaram malhas geográficas uniformes com filtro espacial perto dos polos. Para prevenir a singularidade nos polos na sua totalidade, têm sido desenvolvidas técnicas de discretização alternativas. Uma delas foi usar as projeções gnomônicas ou radiais proposta por Sadourny (1972). A estrategia consiste em decompor a esfera em seis regiões idênticas, obtidas a partir das projeções dos lados de um hexaedro circunscrito na esfera (Figura 2.1b). Cada uma das seis aplicações, isoladamente, não contêm singularidades. No trabalho de Ronchi et al. (1996), a construção foi melhorada e renomeada malha cubada (cubed-sphere). Atualmente é usada em problemas de modelagem atmosférica por exemplo em Harris et al. (2011); Putman e Lin (2007); Scott et al. (2016).

Algumas malhas usadas para discretizar modelos na esfera dependem de certa classe de poliedros altamente simétricos. Em particular, o poliedro conhecido como "bucky" ou "soccer ball", que faz reconhecimento ao arquiteto americano Richard Buckminster Fuller. A malha bucky-ball (Figura 2.1c) apareceu no artigo de Heinze e Hense (2002) para aproximar equações de água rasa na esfera unitária $\mathbb{S}^{2}$. Em particular, a bucky-ball pertence a uma classe de malhas icosaédricas gerais introduzidas por Giraldo (2000) e ademais está classificada com número de malha 3 no sistema dado por Giraldo (1997). Por último, semelhante à esfera cubada, a bucky-ball não possui singularidade nos polos e pode ser gerada a partir de um poliedro inscrito em $\mathbb{S}^{2}$.

\subsection{Malhas esféricas icosaédricas}

As malhas obtidas a partir da projeção radial de poliedros inscritos na esfera são chamadas de malhas geodésicas esféricas. A classe dessas malhas que é gerada a partir dos sólidos platônicos inscritos na esfera, é "ideal" para a construção das malhas geodésicas esféricas uniformes (Figura 2.2). Um sólido platônico é um poliedro regular convexo cujas faces são polígonos onde todos os seus vértices têm o mesmo número de arestas e os seus ângulos são todos congruentes. Porém, devido a construção das malhas esféricas e os seus refinamentos posteriores, os polígonos consequentes irão ter uma leve "distorção" que as vezes prejudica a precisão dos esquemas numéricos (Miura e Kimoto, 2005; Tomita et al., 2001). Dentre os sólidos platônicos, apenas o tetraedro, o octaedro e o icosaedro possuem faces formadas por triângulos equiláteros, e esta característica essencial os torna propícios para a distribuição dos vértices na superfície esférica. Entretanto, o icosaedro é o "melhor" para a construção, pois contém o maior número de vértices e distribui o defeito angular $4 \pi$ uniformemente no número de vértices e minimiza a distorção dos triângulos esféricos perto desses vértices (Wang e Lee, 2011).

O desenvolvimento das malhas esféricas icosaédricas como domínio computacional foi devido principalmente aos trabalhos de Sadourny et al. (1968); Williamson (1968) para a previsão numérica do tempo. Os autores obtiveram resultados satisfatórios, mas ganhou uma melhor acolhida com os desenvolvimentos de Baumgardner e Frederickson (1985); Giraldo (1997); Heikes e Randall (1995a) e Thuburn (1997). Nessa ordem de ideias, a malha esférica icosaédrica consta de uma triangulação curvilinear formada pelas faces projetadas radialmente do icosaedro na superfície esférica. Existem duas maneiras de construir a malha: a primeira é mediante uma aproximação recursiva (diádica) e a segunda de forma não-recursiva. Por simplicidade da apresentação e o desenvolvimento desta tese consideramos apenas a recursiva e é descrita a seguir.

\subsubsection{Construção recursiva}

Colocamos o icosaedro inscrito em $\mathbb{S}^{2}$, em seguida projetamos radialmente os seus 12 vértices na superfície. A posição do icosaedro na esfera é arbitrária, mas consideramos o posicionamento mais usual na literatura (Giraldo, 1997; Sahr et al., 2003; Satoh, 2013), onde dois dos seus vértices 

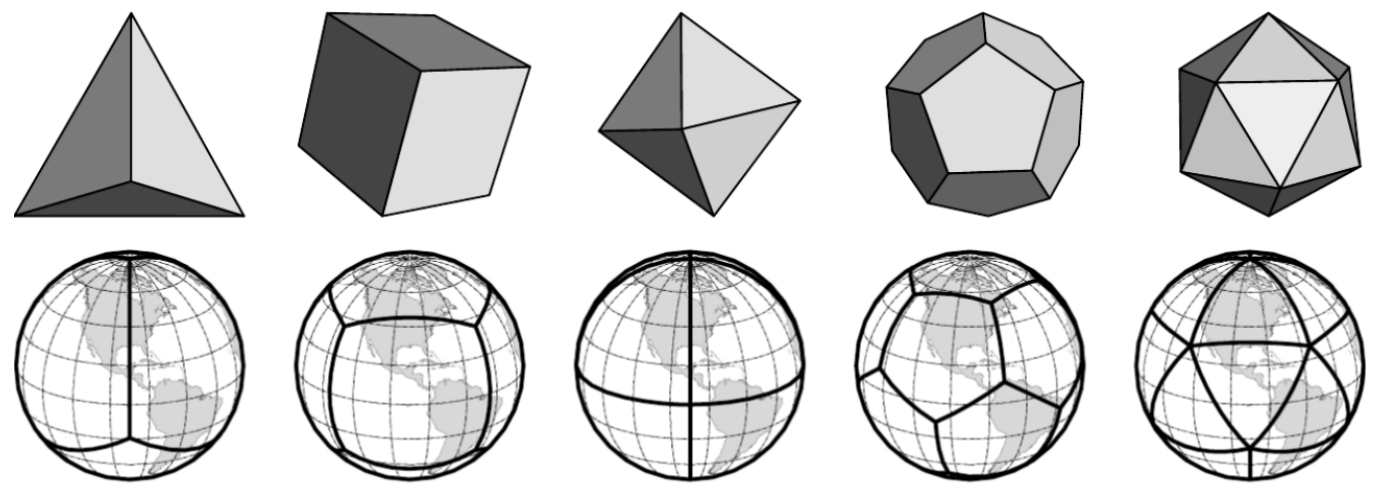

Figura 2.2: Sólidos platónicos e suas respetivas projeções na esfera: tetraedro, hexaedro, octaedro, dodecaedro, icosaedro. Fonte: Gregory et al. (2008).

são colocados em cada polo de $\mathbb{S}^{2}$,

$$
\text { polo norte }:=\left(\phi_{1}, \theta_{1}\right)=\left(\frac{\pi}{2}, 0\right), \quad \text { polo sul }:=\left(\phi_{12}, \theta_{12}\right)=\left(-\frac{\pi}{2}, 0\right),
$$

e os demais, são distribuídos uniformemente na superfície, i.e., com a mesma distância geodésica $\mathrm{d}(\cdot, \cdot)$ entre os vértices adjacentes. Logo, temos cinco no hemisfério norte,

$$
\left(\phi_{i}, \theta_{i}\right):=\left(2 \arcsin \left(\frac{1}{2 \cos \left(\frac{3 \pi}{10}\right)}\right)-\frac{\pi}{2},\left(i-\frac{3}{2}\right) \frac{2 \pi}{5}\right), \quad \text { para } i=2, \ldots, 6,
$$

e os cinco restantes no hemisfério sul,

$$
\left(\phi_{i}, \theta_{i}\right):=\left(-2 \arcsin \left(\frac{1}{2 \cos \left(\frac{3 \pi}{10}\right)}\right)-\frac{\pi}{2},(i-7) \frac{2 \pi}{5}\right), \quad \text { para } i=7, \ldots, 11 .
$$

As 20 faces (triângulos planos equiláteros) do icosaedro são projetadas radialmente na superfície e consequentemente obtemos uma malha triangular composta por triângulos esféricos com arestas geodésicas, conforme ilustrada na Figura 2.3.
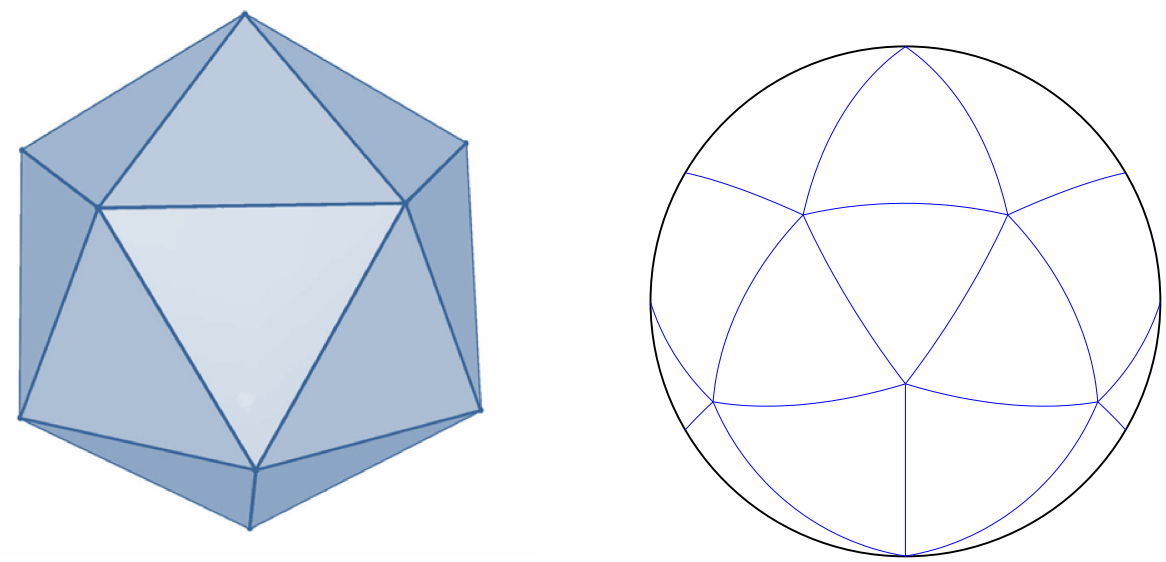

Figura 2.3: $O$ icosaedro e a sua projeção radial na superfície da esfera $\mathbb{S}^{2}$.

A malha inicial pode ser refinada incluindo os pontos médios das arestas triangulares e conectando-os para gerar novos triângulos. Deste modo, cada triângulo é dividido em quatro triângulos esféricos menores no seu interior. Os pontos de bisseção são considerados vértices e são agregados à malha esférica. Por exemplo, se uma aresta geodésica é formada pelos vértices $\mathrm{x}_{i}$ 
e $\mathrm{x}_{j}$, então o ponto médio de $\left[\mathrm{x}_{i}, \mathrm{x}_{j}\right]$ o segmento que liga $\mathrm{x}_{i} \operatorname{com} \mathrm{x}_{j}$, é dado como $\mathrm{x}_{i j}^{*}=\left(\mathrm{x}_{i}+\mathrm{x}_{j}\right) / 2$. Após, é projetado na superfície com a fórmula

$$
\mathrm{x}_{i j}=\frac{\mathrm{x}_{i j}^{*}}{\left\|\mathrm{x}_{i j}^{*}\right\|}
$$

Finalmente, $\mathrm{x}_{i j}$ é definido como um novo vértice na malha. De forma similar, repetimos esse processo nas arestas restantes do triângulo. A construção recursiva é completada quando a média (ou máxima) distância entre pontos de malha adjacentes atinge o valor desejado. Para a construção recursiva o número de vértices, triângulos e arestas são facilmente calculados pelas fórmulas:

$$
\begin{array}{cl}
N=10 \times 2^{2 \ell}+2, & \text { número de vértices, } \\
N_{T}=20 \times 2^{2 \ell}, & \text { número de triângulos, } \\
N_{A}=30 \times 2^{2 \ell}, & \text { número de arestas. }
\end{array}
$$

onde $\ell=0,1,2, \ldots$, é o nível de recursão (ou glevel- $\ell$ como Satoh, 2013; Tomita et al., 2001), que indica o nível de refinamento da malha. Na tabela 2.1 mostramos o número de vértices, triângulos e arestas que caracterizam à malha esférica icosaédrica nos diferentes níveis de refinamento.

\begin{tabular}{cccc}
\hline \multicolumn{4}{c}{ MALHAS ESFÉRICAS ICOSAÉDRICAS } \\
\hline \hline NívEL & VÉRTICES & TRIÂNGULOS & ARESTAS \\
\hline 0 & 12 & 20 & 30 \\
1 & 42 & 80 & 120 \\
2 & 162 & 320 & 480 \\
3 & 642 & 1280 & 1920 \\
4 & 2562 & 5120 & 7680 \\
5 & 10242 & 20480 & 30720 \\
6 & 40962 & 81920 & 122880 \\
7 & 163842 & 327680 & 491520 \\
8 & 655362 & 1310720 & 1966080 \\
9 & 2621442 & 5242880 & 7864320 \\
\hline
\end{tabular}

Tabela 2.1: Niveis de refinamento $\ell$ da malha esférica icosaédrica e seu respectivo número de nós, triângulos e arestas.

Observe-se que o icosaedro é a malha inicial e o seu nível de refinamento é $\ell=0$. Conforme a Figura 2.4, são ilustrados os níveis de refinamento $\ell=0$ até 5 . Além disso, denotando por $N$ o número de vértices, $N_{A}$ o número de arestas e $N_{T}$ o número de faces, temos que o icosaedro satisfaz a característica de Euler:

$$
N=2+N_{A}-N_{T}
$$

\subsubsection{Decomposição Voronoï-Delaunay}

Acompanhando a construção recursiva da malha esférica icosaédrica, definimos o conjunto de vértices distintos por

$$
\mathrm{S}_{N}=\left\{\mathrm{x}_{1}, \ldots, \mathrm{x}_{N}\right\}, \quad \operatorname{com} N=10 \times 2^{2 \ell}+2 .
$$

Temos que o conjunto de triângulos geodésicos forma uma triangulação ou decomposição triangular em $\mathbb{S}^{2}$, denotada por $\widetilde{\mathcal{T}}_{h}\left(\mathbb{S}^{2}\right)$ ou simplesmente $\widetilde{\mathcal{T}}_{h}$, onde $h$ está relacionado ao nível de refinamento da malha e será definido mais tarde. $\widetilde{\mathcal{T}}_{h}$ satisfaz as propriedades:

(i) Os vértices dos triângulos são nós computacionais,

(ii) nenhum triângulo contém outros vértices a não ser seus próprios vértices, 

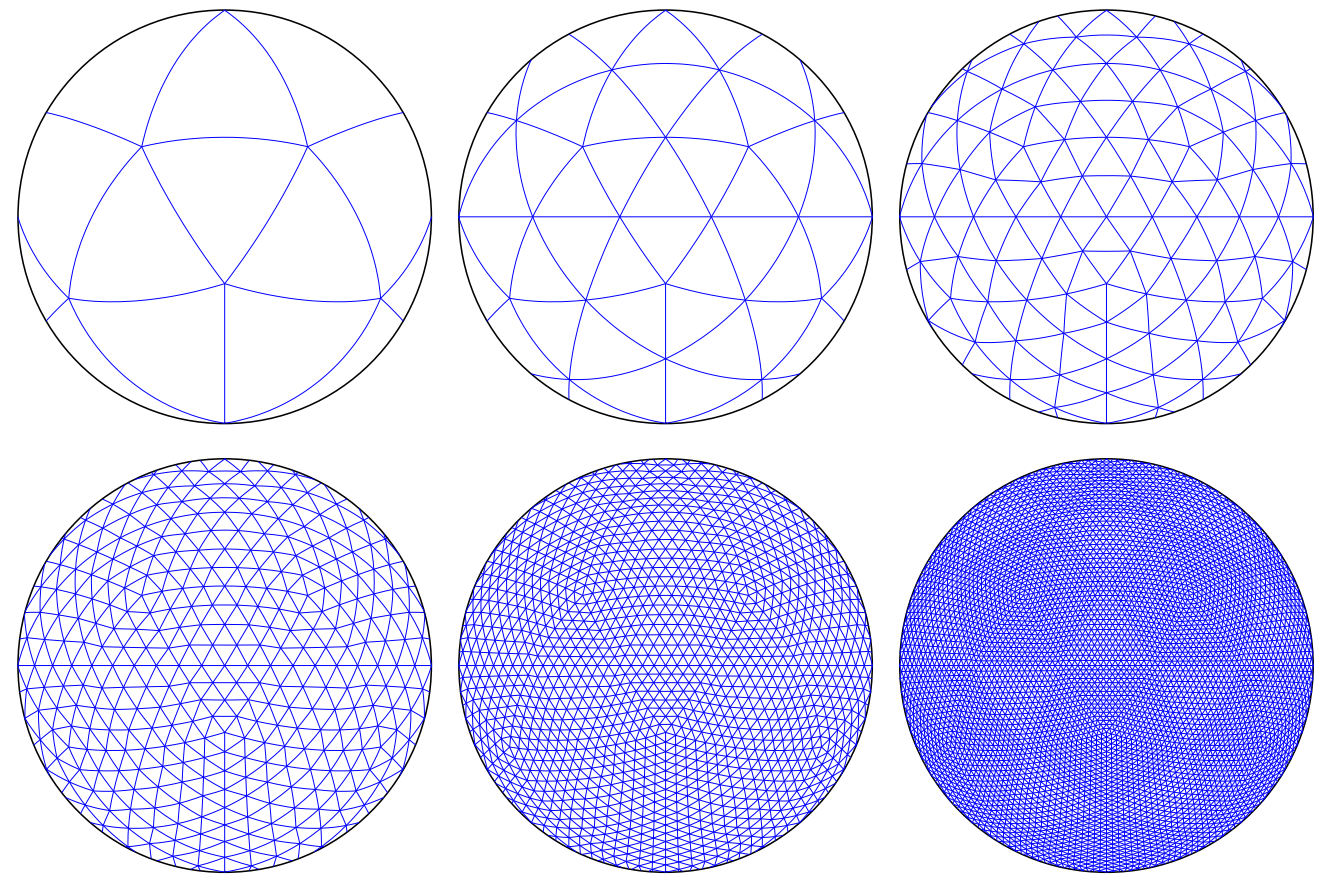

Figura 2.4: Malha esférica icosaédrica com refinamento recursivo de níveis 0 (12 vértices) até 5 (10242 vértices).

(iii) os interiores dos triângulos são dois a dois disjuntos e,

(iv) a união dos triângulos cobre a esfera toda.

Os itens (i)-(ii) são conhecidos na literatura como as condições de uma triangulação tipo conforme para domínios em $\mathbb{R}^{2}$ ou $\mathbb{R}^{3}$ de acordo com Ciarlet (2002). A triangulação $\widetilde{\mathcal{T}}_{h}$, na qual o interior do circuncirculo de qualquer triângulo esférico não contém outro vértice de malha além dos seus três vértices, é denominada como decomposição primal ou triangulação de Delaunay (Gärtner e Kamenski, 2019). Essa decomposição primal tem a propriedade de maximizar o menor de todos os ângulos dos triângulos geodésicos, assim obtemos uma malha triangular esférica com triângulos geodésicos o mais equiláteros possíveis (Hjelle e Dæhlen, 2006; Renka, 1997). Em particular, e para o desenvolvimento desta tese vamos caracterizar a decomposição primal de Delaunay $\widetilde{\mathcal{T}}_{h}$ como segue:

Definição 2.1 (Decomposição primal de Delaunay). Se $\mathrm{x}_{i}, \mathrm{x}_{j}$ e $\mathrm{x}_{k}$ são vértices adjacentes (conectados por arestas) na malha $\widetilde{\mathcal{T}}_{h}$, denotamos por $\widetilde{\mathrm{T}}_{i j k}$ o triângulo geodésico formado pelos vértices $\mathrm{x}_{i}, \mathrm{x}_{j}$ e $\mathrm{x}_{k}$, tal que

$$
\widetilde{\mathcal{T}}_{h}:=\bigcup_{i j k \in \Sigma} \widetilde{\mathrm{T}}_{i j k}
$$

onde $\Sigma=\left\{i j k: i, j, k\right.$ são vizinhos em $\left.\mathrm{S}_{N}\right\}$. Então, $\widetilde{\mathcal{T}}_{h}$ é uma decomposição esférica de Delaunay em $\mathbb{S}^{2}$ associada com os vértices $\mathrm{S}_{N}=\left\{\mathrm{x}_{i}\right\}_{i=1}^{N}$.

Pelo item (iv) acima e da definição anterior, obtemos

$$
\mathbb{S}^{2}=\bigcup_{i j k \in \Sigma} \operatorname{cl}\left(\widetilde{\mathrm{T}}_{i j k}\right)=\bigcup_{\widetilde{T}_{i j k} \in \widetilde{\mathcal{T}}_{h}} \operatorname{cl}\left(\widetilde{\mathrm{T}}_{i j k}\right)
$$

onde $\operatorname{cl}(\cdot)$ denota o fecho do triângulo geodésico $\widetilde{\mathrm{T}}_{i j k} \operatorname{com}$ a métrica $\mathrm{d}(\cdot, \cdot)$ definida em $\mathbb{S}^{2}$.

Observação 2.1. De forma análoga e pela construção recursiva da malha, podemos denotar por $\mathrm{T}_{i j k}$ o triângulo plano da respectiva decomposição $\mathcal{T}_{h}$ associada com os vértices $\mathrm{S}_{N}=\left\{\mathrm{x}_{i}\right\}_{i=1}^{N}$ do poliedro, digamos $\mathbf{S}_{h}$, inscrito em $\mathbb{S}^{2}$. 
Observação 2.2. É importante destacar que para a geração das malhas, bem como para a manipulação e visualização gráfica dos experimentos numéricos, empregamos o código desenvolvido na linguagem FORTRAN9O por Peixoto (2013) da sua tese de doutorado.
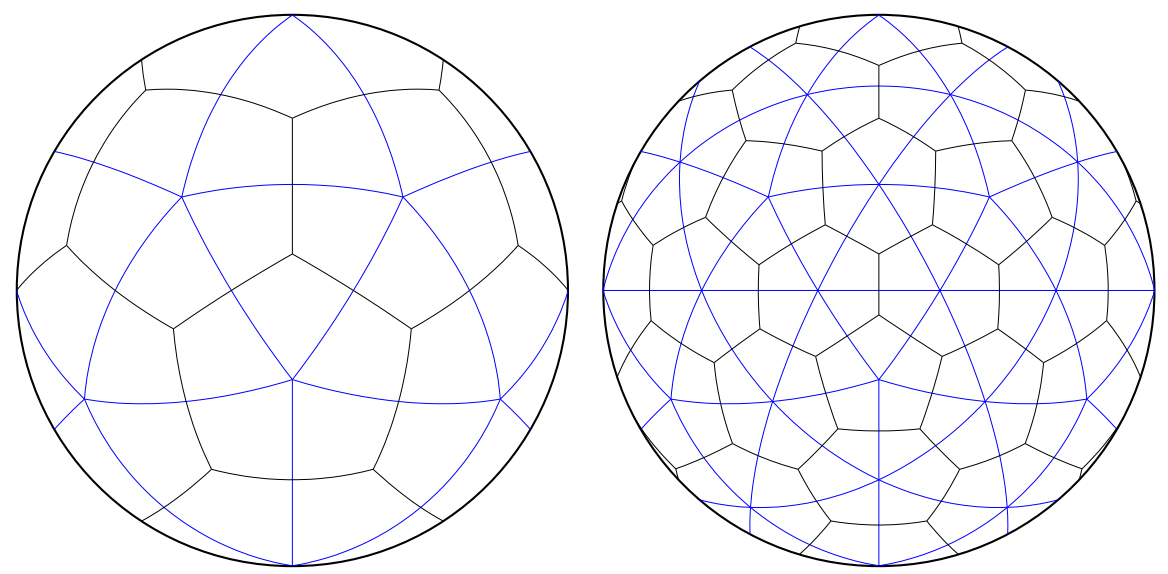

Figura 2.5: Decomposições primal e dual na esfera $\mathbb{S}^{2}$ de níveis 0 e 1.

A decomposição primal $\widetilde{\mathcal{T}}_{h}$ pode ser associada a uma e só uma decomposição dual, que é determinada ao conectar ciclicamente os circuncentros de cada triângulo esférico por geodésicas. Essa nova decomposição é definida como decomposição dual de Voronoï e está constituída pelas regiões de Voronoï, polígonos esféricos formados pelos respectivos circuncentros dos triângulos da decomposição primal (Figura 2.5). Por exemplo, para um triângulo geodésico $\widetilde{\mathrm{T}}_{i j k} \in \widetilde{\mathcal{T}}_{h}$ com vértices $\mathrm{x}_{i}, \mathrm{x}_{j}$ e $\mathrm{x}_{k}$, o seu circuncentro $\mathrm{q}_{i j k}$ é calculado pela expressão

$$
\mathrm{q}_{i j k}=\frac{\left(\mathrm{x}_{j}-\mathrm{x}_{i}\right) \times\left(\mathrm{x}_{k}-\mathrm{x}_{i}\right)}{\left\|\left(\mathrm{x}_{j}-\mathrm{x}_{i}\right) \times\left(\mathrm{x}_{k}-\mathrm{x}_{i}\right)\right\|}
$$

onde $\times$ denota o produto vetorial. Uma outra maneira para construir uma região de Voronoï $\widetilde{\mathrm{V}}_{i}$ em $\mathbb{S}^{2}$, é considerar $\mathrm{H}\left(\mathrm{x}_{i}, \mathrm{x}_{j}\right)$ a região na superfície esférica que contém todos os pontos mais próximos ao ponto $\mathrm{x}_{i}$ do que $\mathrm{x}_{j}$. Assim, a região de Voronoï $\widetilde{\mathrm{V}}_{i}$ será a intersecção das $N-1$ regiões esféricas associadas a cada vértice de $\mathrm{S}_{N}$, i.e.,

$$
\widetilde{\mathrm{V}}_{i}=\bigcap_{\substack{j=1, \ldots, N \\ j \neq i}} \mathrm{H}\left(\mathrm{x}_{i}, \mathrm{x}_{j}\right),
$$

onde cada célula resultante $\widetilde{V}_{i}$ tem pelo menos $N-1$ geodésicas. Quando aplicamos esse processo a cada vértice de $S_{N}$, obtemos um único conjunto de regiões de Voronoï disjuntas $\widetilde{\mathrm{V}}_{i}$ para cada $i=1, \ldots, N$. Introduzimos a definição a seguir.

Definição 2.2 (Região de Voronoï). Seja $\mathrm{S}_{N}=\left\{\mathrm{x}_{i}\right\}_{i=1}^{N}$ um conjunto de vértices distribuídos uniformemente na superfície da esfera unitária $\mathbb{S}^{2}$. Cada vértice $\mathrm{x}_{i} \in \mathrm{S}_{N}$ tem associada uma região de Vorono $\ddot{\mathrm{V}} \widetilde{\mathrm{V}}_{i}$ dada como

$$
\tilde{\mathrm{V}}_{i}:=\tilde{\mathrm{V}}\left(\mathrm{x}_{i}\right)=\left\{\mathrm{x} \in \mathbb{S}^{2}: \mathrm{d}\left(\mathrm{x}, \mathrm{x}_{i}\right)<\mathrm{d}\left(\mathrm{x}, \mathrm{x}_{j}\right), \quad j=1, \ldots, N \text { e } j \neq i\right\}, \quad 1 \leq i \leq N .
$$

O conjunto $\widetilde{\mathrm{V}}_{i}$ consiste de todos os pontos $\mathrm{x}$ em $\mathbb{S}^{2}$ que estão mais próximos a $\mathrm{x}_{i}$ do que qualquer outro ponto de $\mathrm{S}_{N}$.

Observe que todas as regiões de Voronoï são abertas e convexas. Particularmente, dadas duas regiões $\widetilde{\mathrm{V}}_{i}$ e $\widetilde{\mathrm{V}}_{j}$ temos $\widetilde{\mathrm{V}}_{i} \cap \widetilde{\mathrm{V}}_{j}=\emptyset$, para cada $i \neq j$. Cada vértice da decomposição dual de Voronoï está associado a um único triângulo esférico da decomposição primal e esse fato é conhecido como dualidade, conforme o enunciado por Augenbaum e Peskin (1985); Hjelle e Dæhlen (2006), mais precisamente consiste das seguintes características: 
(i) Um vértice de Voronoï corresponde a um triângulo da decomposição primal,

(ii) uma aresta de Voronoï corresponde a uma aresta da decomposição primal,

(iii) uma região de Voronoï corresponde a um vértice na decomposição primal.

Observe que, o circuncentro $\mathrm{q}_{i j k}$ do triângulo $\widetilde{\mathrm{T}}_{i j k} \in \widetilde{\mathcal{T}}_{h}$ com vértices $\mathrm{x}_{i}, \mathrm{x}_{j}$ e $\mathrm{x}_{k}$ está na fronteira das três células de Voronoï associadas a cada um dos vértices do triângulo. Logo, o conjunto dos vértices da decomposição dual de Voronoï está formado unicamente pelos circuncentros dos triângulos da decomposição primal de Delaunay $\widetilde{\mathcal{T}}_{h}$. Por outro lado, denotamos o fecho da célula de Voronoï $\widetilde{\mathrm{V}}_{i}$ por

$$
\operatorname{cl}\left(\widetilde{\mathrm{V}}_{i}\right)=\left\{\mathrm{x} \in \mathbb{S}^{2}: \mathrm{d}\left(\mathrm{x}, \mathrm{x}_{i}\right) \leq \mathrm{d}\left(\mathrm{x}, \mathrm{x}_{j}\right), \quad j=1, \ldots, N\right\}, \quad 1 \leq i \leq N .
$$

Então, a união de todas as regiões $\operatorname{cl}\left(\widetilde{\mathrm{V}}_{i}\right)$, para $i=1, \ldots, N$ cobrem a esfera toda, i.e.,

$$
\mathbb{S}^{2}=\bigcup_{i=1}^{N} \operatorname{cl}\left(\widetilde{\mathrm{V}}_{i}\right)
$$

A decomposição dual de Voronoï associada aos vértices $\mathrm{S}_{N}=\left\{\mathrm{x}_{i}\right\}_{i=1}^{N}$ é definida como:

Definição 2.3 (Decomposição dual de Voronoï). Seja $\mathrm{S}_{N}=\left\{\mathrm{x}_{i}\right\}_{i=1}^{N}$ o conjunto de vértices na esfera unitária $\mathbb{S}^{2}$. Cada vértice em $\mathrm{S}_{N}$ tem associada uma única região $\widetilde{\mathrm{V}}_{i}$, então uma decomposição dual de Voronoï é dada por

$$
\widetilde{\mathcal{V}}=\left\{\mathrm{x}_{i}, \widetilde{\mathrm{V}}_{i}\right\}_{i=1}^{N}
$$
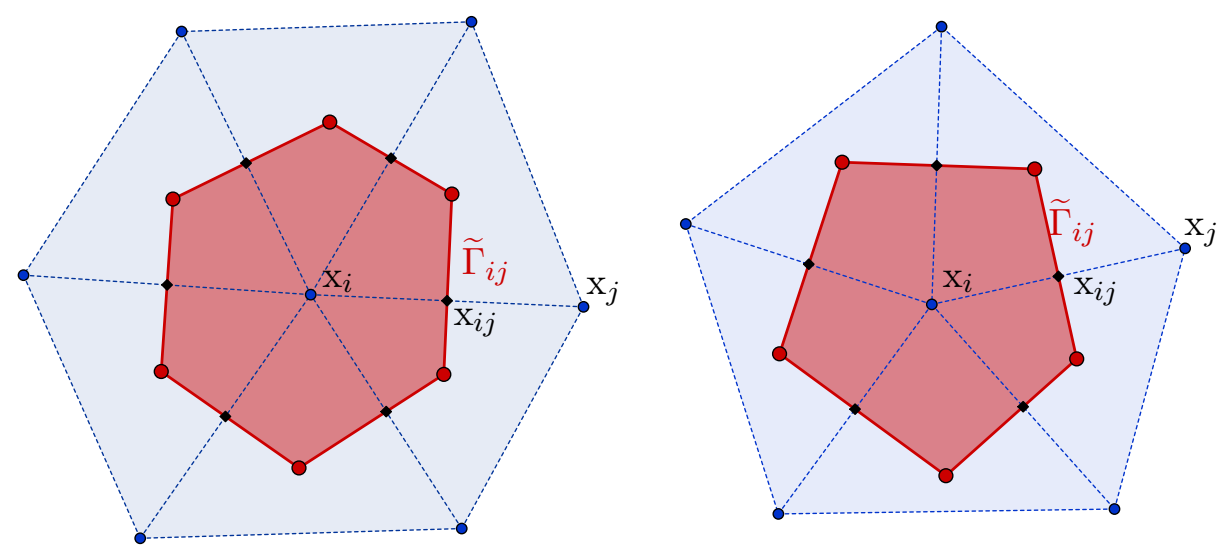

Figura 2.6: Configuração geométrica das células $\widetilde{\mathrm{V}}_{i}$ (hexágono/pentágono) associadas ao vértice $\mathrm{x}_{i}$, onde $\mathrm{x}_{i j}$ é o ponto médio da aresta geodésica $\mathrm{d}\left(\mathrm{x}_{i}, \mathrm{x}_{j}\right)$ e $\widetilde{\Gamma}_{i j}$ a aresta comum entre as células de Voronoï associadas aos vértices $\mathrm{x}_{i}$ e $\mathrm{x}_{j}$ respectivamente.

Em particular, no icosaedro esférico, a decomposição dual de Voronoï é formada por pentágonos/hexágonos. Notamos que, no refinamento da malha, os pentágonos ficam localizados nos 12 vértices iniciais e os hexágonos nos vértices restantes, conforme é ilustrado na Figura 2.5. É importante destacar, que esses polígonos esféricos, sejam pentágonos ou hexágonos, serão usados como volumes de controle nos MVF descritos no Capítulo 3.

Observação 2.3. A decomposição Voronö̈-Delaunay construída anteriormente será considerada como a malha pura sem otimização e será denotada ao longo do trabalho como NOPT.

Cabem agora algumas considerações para esse desenvolvimento. Em primeiro lugar, o par de vértices $\mathrm{x}_{i}$ e $\mathrm{x}_{j}$ são vizinhos se e só se $\widetilde{\Gamma}_{i j}=\operatorname{cl}\left(\widetilde{\mathrm{V}}_{i}\right) \cap \operatorname{cl}\left(\widetilde{\mathrm{V}}_{j}\right) \neq \emptyset$, i.e., para a decomposição dual de 
Voronoï, $\widetilde{\Gamma}_{i j}$ é uma aresta geodésica em $\mathbb{S}^{2}$ sempre que o cumprimento $m\left(\widetilde{\Gamma}_{i j}\right)$ de $\widetilde{\Gamma}_{i j}$ estiver bem definido (Figura 2.6). Com esta notação podemos estabelecer para cada $\mathrm{x}_{i}$ o conjunto de índices dos seus vizinhos $\mathrm{x}_{j}$ tal que $m\left(\widetilde{\Gamma}_{i j}\right)>0$, i.e.,

$$
\Lambda(i)=\left\{j: j \neq i \text { е } \widetilde{\Gamma}_{i j}=\operatorname{cl}\left(\widetilde{\mathrm{V}}_{i}\right) \cap \operatorname{cl}\left(\widetilde{\mathrm{V}}_{j}\right) \neq \emptyset\right\} .
$$

Cada $\widetilde{\mathrm{V}}_{i}$ possui fronteira suave por partes $\partial \widetilde{\mathrm{V}}_{i}$, formada pelas arestas $\widetilde{\Gamma}_{i j}$, para cada $j \in \Lambda(i)$, tal que

$$
\partial \widetilde{\mathrm{V}}_{i}=\bigcup_{j \in \Lambda(i)} \widetilde{\Gamma}_{i j}
$$

onde $\Lambda(i)$ denota o conjunto de vértices vizinhos do $\mathrm{x}_{i}$.

Algumas outras notações e fatos relevantes na decomposição dual de Voronoï são, para $\mathrm{x}_{i}$ e $\mathrm{x}_{j}$ vértices vizinhos com $j \in \Lambda(i)$ :

(i) Denotamos por $\overrightarrow{\mathrm{x}_{i} \mathrm{x}_{j}}$ o vetor que vai de $\mathrm{x}_{i}$ até $\mathrm{x}_{j}$,

(ii) por $\widetilde{\tau}_{i j}$ a aresta primal do triângulo geodésico $\widetilde{\mathrm{T}}_{i j k}$ que conecta os vértices $\mathrm{x}_{i}$ e $\mathrm{x}_{j}$,

(iii) os pontos $\mathrm{x}_{i j}$ e $\mathrm{m}_{i j}$ denotam os pontos médios das arestas geodésicas $\widetilde{\tau}_{i j}$ (primal) e $\widetilde{\Gamma}_{i j}$ (dual) respectivamente,

(iv) cada aresta primal geodésica $\widetilde{\tau}_{i j}$ é perpendicular à aresta dual $\widetilde{\Gamma}_{i j}$, i.e., $\widetilde{\tau}_{i j} \perp \widetilde{\Gamma}_{i j}$,

(v) o plano formado por $\widetilde{\Gamma}_{i j}$ e a origem, divide à aresta $\widetilde{\tau}_{i j}$ no seu ponto médio $\mathrm{x}_{i j}$, então

$$
\mathrm{d}\left(\mathrm{x}_{i}, \mathrm{x}\right)=\mathrm{d}\left(\mathrm{x}, \mathrm{x}_{j}\right), \quad \text { para cada } \mathrm{x} \in \widetilde{\Gamma}_{i j}
$$

(vi) e $\vec{n}_{\mathrm{x}, \widetilde{\Gamma}_{i j}}$ é o vetor normal unitário na aresta de $\widetilde{\Gamma}_{i j}$ que pertence ao plano $\mathbb{T}_{\mathbb{S}^{2}, \mathrm{x}}$ tangente à esfera no ponto x. O vetor $\overrightarrow{\mathrm{n}}_{\mathrm{x}, \widetilde{\Gamma}_{i j}}$ é paralelo a $\overrightarrow{\mathrm{x}}_{i} \mathrm{x}_{j}$, i.e., $\overrightarrow{\mathrm{n}}_{\mathrm{x}, \widetilde{\Gamma}_{i j}} \| \overrightarrow{\mathrm{x}}_{i} \mathrm{x}_{j}$ para cada $\mathrm{x} \in \widetilde{\Gamma}_{i j}$.

A metodologia usada na construção das decomposições Voronoï-Delaunay permite implementar tanto Métodos de Volumes Finitos como de Elementos Finitos para a equação de Laplace. Uma característica essencial para a discretização da equação e a sua implementação pelo MVF é a bisseção perpendicular das arestas dadas nos itens (iv) e (vi). Em particular, se usamos os dois tipos de decomposições, temos que a decomposição primal permite calcular ou aproximar a solução exata do problema nos seus vértices, enquanto a decomposição dual permite discretizar a equação diferencial em cada uma das suas células computacionais (Lin et al., 2013).

Com base nas definições anteriores, consideramos agora alguns parâmetros referentes às medidas e distâncias entre os vértices da decomposição dual de Voronoï. Uma primeira medida de malha é proposta por Du et al. (2003b) como,

$$
h=\max _{i=1, \ldots, N} h_{i}, \quad h_{i}=\max _{\mathrm{x} \in \widetilde{\mathrm{V}}_{i}} \mathrm{~d}\left(\mathrm{x}_{i}, \mathrm{x}\right),
$$

que relaciona as distâncias geodésicas dos pontos na célula de Voronoï. Sendo assim $h_{i}$ a distância geodésica máxima entre um vértice $\mathrm{x}_{i}$ e os seus pontos na sua célula associada $\widetilde{\mathrm{V}}_{i}$ e $h$ a máxima distância geodésica entre algum vértice e os seus pontos na sua célula. Devido à presença de hexágonos e pentágonos, a decomposição dual torna-se não-uniforme, por conseguinte a medida $h$ pode ser usada como medida de uniformidade de malha, i.e., de quão perto uma malha ideal é uniforme em $\mathbb{S}^{2}$ (Du et al., 2003b). Esse fenômeno de não uniformidade pode ser atribuído à distorção nas células (Peixoto, 2013; Tomita et al., 2001). Além da medida de uniformidade na decomposição dual de Voronoï $\widetilde{\mathcal{V}}=\left\{\mathrm{x}_{i}, \widetilde{\mathrm{V}}_{i}\right\}_{i=1}^{N}$ definimos uma medida de regularidade da malha $\sigma$ relacionada à distância geodésica dos pontos na célula de Voronoï (Du et al., 2003b; Ringler, 2003). 
A medida é dada como

$$
\sigma=\min _{i=1, \ldots, N} \sigma_{i}, \quad \sigma_{i}=\min _{j \in \Lambda(i)} \sigma_{i j} \quad \text { onde } \quad \sigma_{i j}=\frac{\mathrm{d}\left(\mathrm{x}_{i}, \mathrm{x}_{j}\right)}{2 h_{i}} .
$$

Observação 2.4. As malhas esféricas icosaédricas não são malhas "ideais" uniformes (ou seja $\sigma=\sigma_{i}=\sigma_{i j}$ para cada $i$ e $j \in \Lambda(i)$ ). Se existir uma variação na uniformidade das células, o valor de $\sigma$ é reduzido. Então, $\sigma$ pode ser usada como uma outra medida da uniformidade da malha; quanto maior for o valor de $\sigma$ mais uniforme será a malha. $O$ valor de $\sigma$ nos fornece uma medida de uniformidade local da malha (Du et al., 2003b). Em particular, algumas demonstrações dos resultados desta tese dependerão do parâmetro $\sigma$, e para esses casos, vamos dizer que existem constantes positivas que dependem do valor da regularidade da malha. Por outro lado, conforme a dualidade na construção recursiva da malha, a decomposição primal dependerá também deste parâmetro $\sigma$.

\subsection{Otimização}

Antes de começar a exposição da otimização, convém esclarecer sua necessidade. Conforme descrito por Heikes e Randall (1995b), a malha esférica icosaédrica original apresenta deficiências na precisão de certos operadores diferenciais. Em geral, se temos malhas de qualidade "ruim", i.e., se as células das malhas são muito distorcidas, vão produzir fontes de erros de truncamento grandes que dificultam a obtenção de estimativas do erro das soluções aproximadas. Não pretendemos elaborar alguma melhora nas malhas, mas sim fazer uma breve comparação no sentido prático. Certas características geométricas como colocação, bisseção e ortogonalidade foram discutidas em diferentes contextos por Miura e Kimoto (2005); Ringler (2003) e Peixoto (2013).

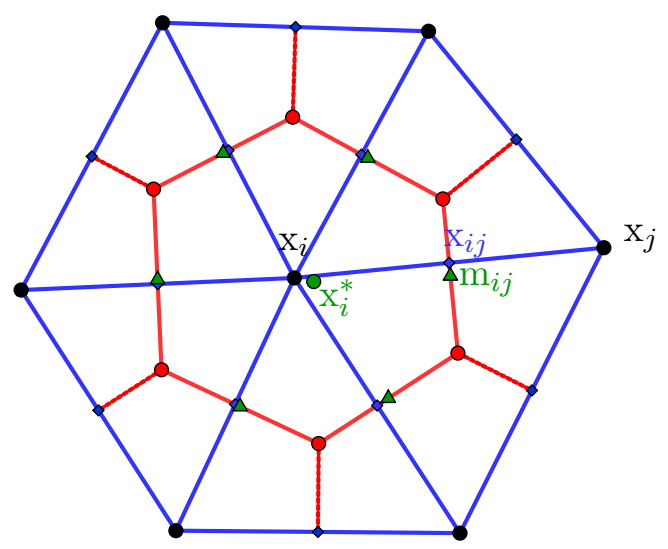

Figura 2.7: Configuração geométrica dos pontos médios das arestas da decomposição primal (destacados em quadrados azuis) e os pontos médios das arestas da decomposição dual (destacados em triângulos verdes). Além, o centroide $\mathrm{x}_{i}^{*}$ (destacado no circulo verde) da célula de Voronö̈ associada ao vértice $\mathrm{x}_{i}$.

\subsubsection{Malha esférica centroidal de Voronoï (SCVT)}

A discretização proposta por Du et al. (2003a), centra-se em decomposições duais de Voronoï e é denominada na literatura como esférica centroidal de Voronoï (do inglês Spherical Centroidal Voronoï Tessellation) e será denotada ao longo desta tese por SCVT. Nas malhas esféricas icosaédricas em geral, os vértices ou centros associados as células de Voronoï não necessariamente coincidem com os seus centroides esféricos (Figura 2.7). O objetivo principal da SCVT é ajustar essa distância por meio de um funcional de forçamento ou colocação. Consideramos primeiramente o seguinte fato: o centroide usual no $\mathbb{R}^{3}$ de um polígono esférico $\widetilde{\mathrm{V}}$ pode ser calculado por

$$
\mathrm{x}^{*}=\int_{\widetilde{\mathrm{V}}} \mathrm{x} d s(\mathrm{x})
$$


Em geral, $\mathrm{x}^{*}$ não está na superfície da esfera, mas sim dentro dela. Consideramos então a definição descrita por Du et al. (2003a) para o centroide restrito a $\mathbb{S}^{2}$.

Definição 2.4 (Centroide esférico). Dado um polígono esférico $\widetilde{\mathrm{V}}$, o centroide esférico $\mathrm{x}^{s} \in \mathbb{R}^{3}$ é um ponto tal que $F\left(\mathrm{x}^{s}\right) \leq F(\mathrm{x})$ para cada $\mathrm{x} \in \tilde{\mathrm{V}}$ com

$$
F(\mathrm{x})=\int_{\widetilde{\mathrm{V}}}\|\mathrm{x}-\mathrm{y}\|_{\mathbb{R}^{3}}^{2} d s(\mathrm{y}),
$$

onde $\|\cdot\|_{\mathbb{R}^{3}}$ é a norma euclidiana.

Com $\mathbb{S}^{2}$ na origem, temos que o centroide esférico de qualquer célula definida na superfície é obtido a partir do centroide padrão no $\mathbb{R}^{3}$ como,

$$
\mathrm{x}^{c}=\frac{\mathrm{x}^{*}}{\left\|\mathrm{x}^{*}\right\|_{\mathbb{R}^{3}}} .
$$

Em geral, a otimização de Du et al. (2003a) consiste em forçar ou colocar o vértice associado à célula de Voronoï para ser o centroide da célula, preservando a ortogonalidade entre as arestas primal e dual (Miura e Kimoto, 2005). Agora, dada uma função de densidade $\rho(\mathrm{x})>0$ para cada $\mathrm{x} \in \mathbb{S}^{2}$, temos que para cada região $\widetilde{\mathrm{V}}_{i} \subset \mathbb{S}^{2}$, o centroide padrão $\mathrm{x}_{i}^{*}$ de $\widetilde{\mathrm{V}}_{i}$ é calculado pela expressão

$$
\mathrm{x}_{i}^{*}=\frac{\int_{\widetilde{\mathrm{V}}_{i}} \mathrm{x} \rho(\mathrm{x}) d s(\mathrm{x})}{\int_{\widetilde{\mathrm{V}}_{i}} \rho(\mathrm{x}) d s(\mathrm{x})}, \quad \text { para } i=1, \ldots, N .
$$

Observe que, $\mathrm{x}_{i}^{*}$ não está em $\mathbb{S}^{2}$, mas sim dentre dela. A distância entre o centroide padrão $\mathrm{x}_{i}^{*}$ e o centroide $\mathrm{x}_{i}^{c}$ restrito a $\mathbb{S}^{2}$ é definida a seguir.

Proposição 2.1 (Du et al., 2003a). Sejam $\mathrm{x}_{i}^{c} \in \mathbb{S}^{2}$ e $\mathrm{x}_{i}^{*} \in \mathbb{R}^{3}$ os centroides restrito a $\mathbb{S}^{2}$ e padrão da célula $\widetilde{\mathrm{V}}_{i}$, respectivamente. Então, $\mathrm{x}_{i}^{*}-\mathrm{x}_{i}^{c}$ é um vetor normal à superfície de $\mathbb{S}^{2}$ no ponto $\mathrm{x}_{i}^{c}$, i.e., $\mathrm{x}_{i}^{c}$ é a projeção radial de $\mathrm{x}_{i}^{*}$ em $\mathbb{S}^{2}$ a longo da direção normal em $\mathrm{x}_{i}^{c}$.

Além disso, o centroide restrito $\mathrm{x}_{i}^{c}$ de uma região esférica $\widetilde{\mathrm{V}}_{i}$ é a solução ao problema:

$$
\min _{\mathrm{x} \in \widetilde{\mathrm{V}}_{i}} F(\mathrm{x}), \quad \text { onde } F(\mathrm{x})=\int_{\widetilde{\mathrm{V}}_{i}} \rho(\mathrm{y})\|\mathrm{x}-\mathrm{y}\|_{\mathbb{R}^{3}}^{2} d s(\mathrm{y}) .
$$

Em consequência, dizemos que uma decomposição de Voronoï é do tipo SCVT se e só se, os vértices $\mathrm{S}_{N}=\left\{\mathrm{x}_{i}\right\}_{i=1}^{N}$ associados as regiões de Voronoï são também os centroides restritos dessas regiões, i.e., $\mathrm{x}_{i}=\mathrm{x}_{i}^{c}$. Assim, dada uma decomposição de Voronoï $\left\{\mathrm{x}_{i}^{\#}, \widetilde{\mathrm{V}}_{i}^{\#}\right\}_{i=1}^{N}$ de $\mathbb{S}^{2}$, podemos definir o seguinte funcional de energia (clustering energy):

$$
\mathcal{K}\left(\left\{\mathrm{x}_{i}^{\#}, \widetilde{\mathrm{V}}_{i}^{\#}\right\}_{i=1}^{N}\right)=\sum_{i=1}^{N} \int_{\widetilde{\mathrm{V}}_{i}^{\#}} \rho(\mathrm{y})\left\|\mathrm{y}-\mathrm{x}_{i}^{\#}\right\|_{\mathbb{R}^{3}}^{2} d s(\mathrm{y})
$$

Esse funcional de energia é usado como medida de qualidade da malha e é minimizado sempre que $\left\{\mathrm{x}_{i}^{\#}, \widetilde{\mathrm{V}}_{i}^{\#}\right\}_{i=1}^{N}$ seja uma SCVT e é frequentemente associado com quantidades de variância e erro de quantização, de acordo relatado por Du et al. (2003a). Além disso, se no funcional de energia a função de densidade é constante obteremos uma malha SCVT uniformemente distribuída em $\mathbb{S}^{2}$. Caso contrário, para funções não constantes fornece uma distribuição não uniforme dos vértices, enquanto a acumulação dos vértices da SCVT permanece localmente regular (Du et al., 2003b, 2010). Não obstante, para o desenvolvimento dos experimentos numéricos da tese será usada uma função de densidade constante $\rho(\mathrm{x})>0$ para cada $\mathrm{x} \in \mathbb{S}^{2}$.

Existem várias formas para construir malhas SCVT (vide Du et al., 2003a; Ju et al., 2002). Em particular, conforme os resultados obtidos por Du et al. (2003b) e Du e Gunzburger (2002), enunciamos o algoritmo determinístico de Lloyd para gerar a SCVT. 
Algoritmo 2.1. (Método de Lloyd) Dada uma função de densidade $\rho(\mathrm{x})$ definida para cada $\mathrm{x} \in \mathbb{S}^{2}$ e $N$ um inteiro positivo,

1: Defina o conjunto de $N$ vértices $\mathrm{S}_{N}:=\left\{\mathrm{x}_{i}\right\}_{i=1}^{N}$ em $\mathbb{S}^{2}$

2: Construir as células $\left\{\widetilde{\mathrm{V}}_{i}\right\}_{i=1}^{N}$ de $\mathbb{S}^{2}$ associado com $\left\{\mathrm{x}_{i}\right\}_{i=1}^{N}$;

3: Determinar os centroides restritos do conjunto $\left\{\widetilde{\mathrm{V}}_{i}\right\}_{i=1}^{N}$;

4: Atualizar: os centroides restritos formam um novo conjunto de vértices $\left\{\mathrm{x}_{i}\right\}_{i=1}^{N}$;

5: se o novo conjunto de vértices atinge algum critério de convergência "STOP", caso contrario voltar ao passo 2 .

A SCVT carregar uma vantagem adicional, pode ser gerada sem restrição em qualquer nível de refinamento da malha (Du et al., 2003a) e é de bastante interesse nos modelos globais da previsão do tempo.

\subsubsection{Proposta de Heikes \& Randall (HR95)}

Mencionamos uma outra otimização clássica das malhas esféricas icosaédricas. Consideramos o seguinte fato: observe na Figura 2.7 que os pontos médios das arestas da decomposição primal não coincidem com os pontos médios das arestas da decomposição dual. A proposta de Heikes e Randall (1995b) consiste em minimizar globalmente as distâncias entre esses pontos médios (bisseção) preservando a ortogonalidade entre as arestas. Nesse caso, a condição de colocação da SCVT não sempre é satisfeita devido à distorção nas células. Os autores mostraram que a relação entre os pontos médios e o comprimento da aresta dado como

$$
r_{i j}=\frac{\left(\mathrm{x}_{i j}-\mathrm{m}_{i j}\right)}{m\left(\widetilde{\Gamma}_{i j}\right)}
$$

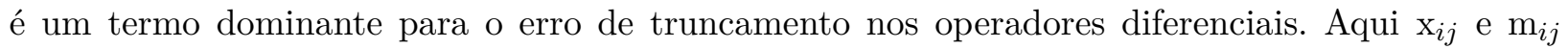
denotam os pontos médios das arestas primal e dual respectivamente e $m\left(\widetilde{\Gamma}_{i j}\right)$ é o comprimento da aresta dual. Então, para caracterizar a optimização, inicialmente foi definida uma função global, mais precisamente a soma das quartas potências dos números $r_{i j}$,

$$
R=\sum_{i=1}^{N} \sum_{j \in \Lambda(i)} r_{i j}^{4} .
$$

O objetivo da optimização é encontrar a distribuição dos vértices que minimiza $R$ mediante um método Quase-Newton. Em outras palavras, o método de optimização não-linear minimiza a função $R$ para ajustar a intersecção das arestas, modificando a bisseção das arestas primal e dual, e além, preservando a ortogonalidade entre elas. Para simplificar a notação, esta malha será denotada por HR95, e mencionada só no contexto dos experimentos numéricos, dado que até o momento não existe um análise teórico de convergência para esta tipo de otimização.

Para finalizar esta seção, dadas as medidas de uniformidade e regularidade, afirmamos que todas as malhas mencionadas acima são em geral, uma classe de decomposições quase-uniformes (não muito distorcidas) de $\mathbb{S}^{2}$. Segue uma definição usual desta classe de malhas, dada por exemplo em Li et al. (2000) ou Mishev (1998) para discretizações de domínios no plano, mas são estendidas de maneira natural em superfícies (Ju e Du, 2009).

Definição 2.5 (Malhas quase-uniformes). Uma família de malhas esféricas icosaédricas $\widetilde{\mathcal{V}}\left(\mathbb{S}^{2}\right)=$ $\left\{\mathrm{x}_{i}, \widetilde{\mathrm{V}}_{i}\right\}_{i=1}^{N}$ de $\mathbb{S}^{2}$ é dita quase-uniforme (não muito distorcida), se para cada polígono esférico $\widetilde{\mathrm{V}}_{i} \in \mathcal{V}$, com fronteira $\partial \widetilde{\mathrm{V}}_{i}=\bigcup_{j \in \Lambda(i)} \widetilde{\Gamma}_{i j}$, existem constantes $\left\{C_{i}\right\}_{i=1}^{3} \subset \mathbb{R}_{+}$, independentes do 
parâmetro $h$, tal que

$$
\begin{aligned}
\frac{1}{C_{1}} h & \leq m\left(\widetilde{\Gamma}_{i j}\right) \leq C_{1} h, \\
\frac{1}{C_{2}} h^{2} & \leq S\left(\widetilde{\mathrm{V}}_{i}\right) \leq C_{2} h^{2}, \\
\operatorname{Card}\left(\partial \widetilde{\mathrm{V}}_{i}\right) & \leq C_{3},
\end{aligned}
$$

onde $\operatorname{Card}\left(\partial \widetilde{\mathrm{V}}_{i}\right)$ denota a quantidade de arestas que constituem a fronteira de $\widetilde{\mathrm{V}}_{i}$.

Para terminar, dizemos então, que as malhas NOPT, SCVT e HR95 são descomposições quase-uniformes, i.e., satisfazem as condições (2.5a)-(2.5c). Tomaremos essencial esta ideia para a obtenção das estimativas de convergência do problema de Laplace em $\mathbb{S}^{2}$. Por último, definido o domínio computacional o passo a seguir será definir uma discretização do Laplaciano associada as decomposições Voronoï-Delaunay de $\mathbb{S}^{2}$. 


\section{Capítulo 3}

\section{Discretização do Laplaciano}

Neste capítulo vamos nos concentrar numa aproximação clássica da equação de Poisson através do Método dos Volumes Finitos (MVF). Inicialmente, formalizaremos esta discretização via um esquema de volumes de diferença finita centrada (Cell-Centered Finite Volume Difference Scheme), conforme a construção da decomposição Voronoï-Delaunay em $\mathbb{S}^{2}$ do capítulo precedente e ademais, o esquema resultante satisfaz a propriedade de conservação.

Em seguida, discutiremos brevemente que, em geral, o MVF é assintoticamente inconsistente, ou seja, o erro local de truncamento do método não tende a zero com o refinamento das malhas. Isso é devido à não uniformidade das células e às aproximações dos fluxos em cada uma das arestas como relatado por Heikes e Randall (1995b) e (Ringler, 2003). Mostraremos que devido à decomposição Voronoï-Delaunay ser quase-uniforme e o esquema ser conservativo, o MVF satisfaz uma condição de consistência fraca nas $L^{2}$ e max-normas no sentido das diferenças finitas (Coudière et al., 1999; Eymard et al., 2000; Faille, 1992), i.e., os fluxos contínuos possuem aproximações consistentes nas arestas da célula. Finalizaremos essa parte com alguns experimentos numéricos do erro de truncamento.

Para garantir as estimativas de convergência do Capítulo 4 é necessário estabelecer condições de estabilidade. Para esse fim, estruturaremos conforme a literatura, uma correspondência geométrica entre $\mathbb{S}^{2}$ e o seu poliedro de aproximação $\mathbf{S}_{h}$. A ideia fundamental é reescrever o MVF como um MEFL e definir um operador que interpole os espaços de aproximação associados à decomposição Voronoï-Delaunay, e em seguida estabelecer algumas estimativas referentes à estabilidade. Por fim, estimamos os erros devidos à aproximação geométrica da esfera pelo poliedro nas formas variacionais correspondentes.

\subsection{Métodos dos volumes finitos}

Dada a decomposição dual de Voronoï $\tilde{\mathcal{V}}=\left\{\mathrm{x}_{i}, \widetilde{\mathrm{V}}_{i}\right\}_{i=1}^{N}$, o MVF consiste em discretizar diretamente a forma integral de (1.4) em cada uma das células de $\widetilde{\mathcal{V}}=\left\{\mathrm{x}_{i}, \widetilde{\mathrm{V}}_{i}\right\}_{i=1}^{N}$. Em seguida, fazendo uso da fórmula de Green, obtemos uma integral de linha do gradiente tangencial na fronteira da célula. Suponhamos que $u: \mathbb{S}^{2} \rightarrow \mathbb{R}$ seja de classe $C^{4}$ e satisfaça,

$$
-\int_{\widetilde{\mathrm{V}}_{i}} \Delta_{s} u(\mathrm{x}) d s(\mathrm{x})=-\int_{\partial \widetilde{\mathrm{V}}_{i}} \nabla_{s} u(\mathrm{x}) \cdot \overrightarrow{\mathrm{n}}_{\mathrm{x}, \widetilde{\mathrm{V}}_{i}} d \gamma(\mathrm{x})=\int_{\widetilde{\mathrm{V}}_{i}} f(\mathrm{x}) d s(\mathrm{x}),
$$

onde $\overrightarrow{\mathrm{n}}_{\mathrm{x}, \widetilde{\mathrm{V}}_{i}}$ é o vetor normal unitário à fronteira $\partial \widetilde{\mathrm{V}}_{i}$ no ponto x e está contido em $\mathbb{T}_{\mathbb{S}^{2}, \mathrm{x}}$, o plano tangente a $\mathbb{S}^{2}$ no ponto $\mathrm{x}$. Agora, queremos estimar o valor do Laplaciano de $u$ no vértice $\mathrm{x}_{i}$ associado com $\widetilde{\mathrm{V}}_{i}$. Expomos esta estimação a seguir.

Proposição 3.1. Sejam $u: \mathbb{S}^{2} \rightarrow \mathbb{R}$ uma função de classe $C^{4}$ e $\widetilde{\mathrm{V}}_{i}$ uma célula da decomposição dual de Voronoï $\widetilde{\mathcal{V}}=\left\{\mathrm{x}_{i}, \widetilde{\mathrm{V}}_{i}\right\}_{i=1}^{N}$ que satisfaz a condição (2.5b). Então, existe uma constante $C \in \mathbb{R}_{+}$ que independe do parâmetro $h$, tal que

$$
\left|\Delta_{s} u\left(\mathrm{x}_{i}^{c}\right)-\frac{1}{S\left(\widetilde{\mathrm{V}}_{i}\right)} \int_{\partial \widetilde{\mathrm{V}}_{i}} \nabla_{s} u(\mathrm{x}) \cdot \overrightarrow{\mathrm{n}}_{\mathrm{x}, \widetilde{\mathrm{V}}_{i}} d \gamma(\mathrm{x})\right| \leq C h^{2},
$$

onde $\mathrm{x}_{i}^{c}$ é o centroide esférico da célula $\widetilde{\mathrm{V}}_{i}, S\left(\widetilde{\mathrm{V}}_{i}\right)$ denota a área superficial da célula e h é o tamanho 
de malha definido em (2.1).

Demonstração. Com efeito, dadas $u \in C^{4}\left(\mathbb{S}^{2}\right)$ e $\widetilde{\mathrm{V}}_{i} \in \tilde{\mathcal{V}}=\left\{\mathrm{x}_{i}, \widetilde{\mathrm{V}}_{i}\right\}_{i=1}^{N}$, tomemos $\left.u\right|_{\widetilde{\mathrm{V}}_{i}} \in C^{4}\left(\widetilde{\mathrm{V}}_{i}\right)$ a restrição de $u$ à célula $\widetilde{\mathrm{V}}_{i}$. Também, consideremos a sua extensão $u^{\Omega}$ ao conjunto $\Omega_{2}:=$ $\left\{\mathrm{x} \in \mathbb{R}^{3}: \frac{1}{2} \leq\|\mathrm{x}\| \leq 2\right\}$ que também é de classe $C^{4}$ em $\Omega_{2}$. Desta maneira, podemos usar o Teorema de Taylor até ordem dois do termo $\Delta u^{\Omega}$, em torno do centroide não restrito à esfera $\mathrm{x}_{i}^{*}$ de $\widetilde{\mathrm{V}}_{i}$, após integrando em relação à $\widetilde{\mathrm{V}}_{i}$, dividindo a expressão pela respectiva área superficial de $\widetilde{\mathrm{V}}_{i}$ e em virtude da condição (2.5b), que diz: existe uma constante $C \in \mathbb{R}_{+}$, independente de $h$, tal que $C h^{2} \leq S\left(\widetilde{\mathrm{V}}_{i}\right)$. Resulta,

$$
\frac{1}{S\left(\widetilde{\mathrm{V}}_{i}\right)} \int_{\widetilde{\mathrm{V}}_{i}} \Delta u^{\Omega}(\mathrm{x}) d s(\mathrm{x})=\Delta u^{\Omega}\left(\mathrm{x}_{i}^{*}\right)+\nabla\left(\Delta u^{\Omega}\left(\mathrm{x}_{i}^{*}\right)\right) \frac{1}{S\left(\widetilde{\mathrm{V}}_{i}\right)} \cdot \int_{\widetilde{\mathrm{V}}_{i}}\left(\mathrm{x}-\mathrm{x}_{i}^{*}\right) d s(\mathrm{x})+R_{i j},
$$

onde $\left|R_{i j}\right| \leq C h^{2} \max _{\mathrm{x} \in \widetilde{\mathrm{V}}_{i}}\left|H\left(\Delta u^{\Omega}(\mathrm{x})\right)\right|$ e $H$ denota a Hessiana de $\Delta u^{\Omega}$ no ponto x. Da definição do centroide no $\mathbb{R}^{3}, \mathrm{x}_{i}^{*}=\frac{1}{S\left(\widetilde{\mathrm{V}}_{i}\right)} \int_{\widetilde{\mathrm{V}}_{i}} \mathrm{x} d s(\mathrm{x})$, o segundo termo da integral do lado direito é zero. Agora, para $\mathrm{x}_{i}^{*} \in \Omega_{2}$, teremos que

$$
\Delta u^{\Omega}\left(\mathrm{x}_{i}^{*}\right)=\Delta_{s} u\left(\frac{\mathrm{x}_{i}^{*}}{\left\|\mathrm{x}_{i}^{*}\right\|}\right)=\Delta_{s} u\left(\mathrm{x}_{i}^{c}\right)
$$

onde $\mathrm{x}_{i}^{c}$ denota o centroide esférico de $\widetilde{\mathrm{V}}_{i}$, ademais $\Delta u^{\Omega}(\mathrm{x})=\Delta_{s} u(\mathrm{x})$ para cada $\mathrm{x} \in \widetilde{\mathrm{V}}_{i}$. Portanto,

$$
\frac{1}{S\left(\widetilde{\mathrm{V}}_{i}\right)} \int_{\widetilde{\mathrm{V}}_{i}} \Delta_{s} u(\mathrm{x}) d s(\mathrm{x})=\Delta_{s} u\left(\mathrm{x}_{i}^{c}\right)+R_{i j}
$$

Finalmente, a limitação desejada segue imediatamente da fórmula de Green (3.1) e observando que a constante depende apenas das segundas derivadas de $\Delta u^{\Omega}$. O que prova a proposição.

Como relatado na Subseção 2.2.2, uma célula de Voronoï $\widetilde{\mathrm{V}}_{i}$ é um polígono esférico aberto convexo com fronteira suave por pedaços. Sua fronteira $\partial \widetilde{\mathrm{V}}_{i}$ pode ser decomposta em um conjunto de arestas $\widetilde{\Gamma}_{i j}$ tal que

$$
\partial \widetilde{\mathrm{V}}_{i}=\bigcup_{j \in \Lambda(i)} \widetilde{\Gamma}_{i j}
$$

onde $\Lambda(i)$ é o conjunto de vizinhos do vértice $\mathrm{x}_{i}$. Segundo essa notação, teremos para (3.1) a expressão,

$$
-\int_{\widetilde{\mathrm{V}}_{i}} \Delta_{s} u(\mathrm{x}) d s(\mathrm{x})=-\sum_{j \in \Lambda(i)} \int_{\widetilde{\Gamma}_{i j}} \nabla_{s} u(\mathrm{x}) \cdot \overrightarrow{\mathrm{n}}_{\mathrm{x}, \widetilde{\Gamma}_{i j}} d \gamma(\mathrm{x}) .
$$

onde $\vec{n}_{\mathrm{x}, \widetilde{\Gamma}_{i j}}$ é o vetor normal unitário externo à célula $\widetilde{V}_{i}$ contido no plano tangente $\mathbb{T}_{\mathbb{S}^{2}, \mathrm{x}}$. No que se segue, vamos denotar ao fluxo contínuo da função $u$ através da aresta $\widetilde{\Gamma}_{i j}$ por,

$$
\mathrm{F}_{i j}^{c}(u):=-\int_{\widetilde{\Gamma}_{i j}} \nabla_{s} u(\mathrm{x}) \cdot \overrightarrow{\mathrm{n}}_{\mathrm{x}, \widetilde{\Gamma}_{i j}} d \gamma(\mathrm{x}), \quad \text { para cada } j \in \Lambda(i) .
$$

Um ingrediente básico envolvido na construção da discretização é a determinação de uma aproximação da integral ao longo das arestas $\widetilde{\Gamma}_{i j}$ com base apenas no valor da função no ponto médio $\mathrm{m}_{i j}$ de cada aresta $\widetilde{\Gamma}_{i j}$, conforme ilustrado na Figura 3.1. Mostraremos a seguir, uma estimação do fluxo por um método de integração numérica de ordem três em relação ao cumprimento da aresta.

Proposição 3.2. Sejam $u: \mathbb{S}^{2} \rightarrow \mathbb{R}$ uma função de classe $C^{4}$ e $\widetilde{\Gamma}_{i j}$ uma aresta da célula de Voronoï $\widetilde{\mathrm{V}}_{i}$ associada ao vértice $\mathrm{x}_{i}$ da decomposição dual $\widetilde{\mathcal{V}}=\left\{\mathrm{x}_{i}, \widetilde{\mathrm{V}}_{i}\right\}_{i=1}^{N}$ que satisfaz a condição (2.5a). 


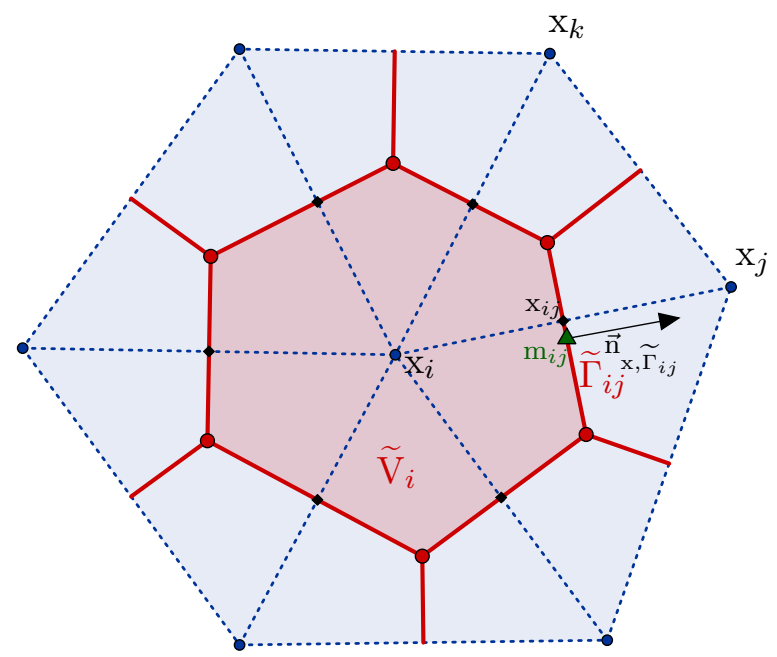

Figura 3.1: Representação do stencil para a obtenção da aproximação do Laplaciano no vértice $\mathrm{x}_{i}$.

Então, existe uma constante $C \in \mathbb{R}_{+}$, que independe do parâmetro $h$, tal que

$$
\left|\mathrm{F}_{i j}^{c}(u)-m\left(\widetilde{\Gamma}_{i j}\right) \nabla_{s} u\left(\mathrm{~m}_{i j}\right) \cdot \overrightarrow{\mathrm{n}}_{\mathrm{x}, \widetilde{\Gamma}_{i j}}\right| \leq C h^{3}
$$

onde $\mathrm{F}_{i j}^{c}$ denota o fluxo contínuo através de $\widetilde{\Gamma}_{i j}, \mathrm{~m}_{i j}$ e $m\left(\widetilde{\Gamma}_{i j}\right)$ denotam respectivamente o ponto médio e o comprimento da aresta e h é o tamanho de malha definido em (2.1).

Demonstração. Com um argumento similar ao dado na prova da Proposição 3.1, basta mostrar para $u \in C^{4}\left(\tilde{\mathrm{V}}_{i}\right)$. Sabemos que para cada $\mathrm{x} \in \mathbb{S}^{2}, u(\mathrm{x})=u^{\Omega}(\mathrm{x})$ e que $\nabla u^{\Omega}(\mathrm{x}) \cdot \overrightarrow{\mathrm{n}}_{\mathbb{S}^{2}, \mathrm{x}}=0$. Resulta da definição do gradiente tangencial que

$$
\nabla_{s} u(\mathrm{x})=\nabla u^{\Omega}(\mathrm{x})-\left(\nabla u^{\Omega}(\mathrm{x}) \cdot \overrightarrow{\mathrm{n}}_{\mathbb{S}^{2}, \mathrm{x}}\right) \overrightarrow{\mathrm{n}}_{\mathbb{S}^{2}, \mathrm{x}}=\nabla u^{\Omega}(\mathrm{x}), \quad \text { para cada } \mathrm{x} \in \mathbb{S}^{2} .
$$

Assim, dados $\mathrm{m}_{i j}$ o ponto médio de $\widetilde{\Gamma}_{i j}$ e o vetor $\overrightarrow{\mathrm{n}}_{\mathrm{x}, \widetilde{\Gamma}_{i j}}$ normal a $\widetilde{\Gamma}_{i j}$ no ponto x, podemos considerar o Teorema de Taylor até ordem dois do termo $\nabla u^{\Omega}(\mathrm{x}) \cdot \overrightarrow{\mathrm{n}}_{\mathrm{x}, \widetilde{\Gamma}_{i j}}$ em torno de $\mathrm{m}_{i j}$, após integrando em relação a $\widetilde{\Gamma}_{i j}$ obteremos

$$
\begin{aligned}
& \int_{\widetilde{\Gamma}_{i j}} \nabla_{s} u(\mathrm{x}) \cdot \overrightarrow{\mathrm{n}}_{\mathrm{x}, \widetilde{\Gamma}_{i j}} d \gamma(\mathrm{x})=\int_{\widetilde{\Gamma}_{i j}} \nabla u^{\Omega}(\mathrm{x}) \cdot \overrightarrow{\mathrm{n}}_{\mathrm{x}, \widetilde{\Gamma}_{i j}} d \gamma(\mathrm{x}) \\
& =m\left(\widetilde{\Gamma}_{i j}\right) \nabla u^{\Omega}\left(\mathrm{m}_{i j}\right) \cdot \overrightarrow{\mathrm{n}}_{\mathrm{x}, \widetilde{\Gamma}_{i j}}+\nabla\left(\nabla u^{\Omega}\left(\mathrm{m}_{i j}\right) \cdot \overrightarrow{\mathrm{n}}_{\mathrm{x}, \widetilde{\Gamma}_{i j}}\right) \cdot \int_{\widetilde{\Gamma}_{i j}}\left(\mathrm{x}-\mathrm{m}_{i j}\right) d \gamma(\mathrm{x}) \\
& +R_{i j}
\end{aligned}
$$

onde $\left|R_{i j}\right| \leq C h^{3} \max _{\mathrm{x} \in \widetilde{\Gamma}_{i j}}\left|H\left(\nabla u^{\Omega}(\mathrm{x}) \cdot \overrightarrow{\mathrm{n}}_{\mathrm{x}, \widetilde{\Gamma}_{i j}}\right)\right|$ e $H$ denota a matriz Hessiana. Observe-se que usamos a condição $(2.5 \mathrm{a})$, i.e., existe uma constante $C \in \mathbb{R}_{+}$independente de $h$, tal que $m\left(\widetilde{\Gamma}_{i j}\right) \leq C h$, o fato $\left\|\mathrm{x}-\mathrm{m}_{i j}\right\| \leq h$. Ora, o segundo termo do lado direito da expressão acima é zero, em virtude da simetria em relação ao ponto médio $\mathrm{m}_{i j}$. Então, resulta

$$
\int_{\widetilde{\Gamma}_{i j}} \nabla_{s} u(\mathrm{x}) \cdot \overrightarrow{\mathrm{n}}_{\mathrm{x}, \widetilde{\Gamma}_{i j}} d \gamma(\mathrm{x})=m\left(\widetilde{\Gamma}_{i j}\right) \nabla u^{\Omega}\left(\mathrm{m}_{i j}\right) \cdot \overrightarrow{\mathrm{n}}_{\mathrm{x}, \widetilde{\Gamma}_{i j}}+R_{i j}
$$

Finalmente, pondo a notação para o fluxo, equação (3.3), obtemos a limitação desejada, notando que a constante irá depender apenas do valor da Hessiana do fluxo.

O resultado anterior permite então, expressar uma aproximação do valor do Laplaciano de $u$ 
num vértice $\mathrm{x}_{i}$ mediante os valores dos fluxos nos pontos médios das arestas da célula de Voronoï associada. Mais propriamente:

Proposição 3.3. Sejam $u: \mathbb{S}^{2} \rightarrow \mathbb{R}$ uma função de classe $C^{4}$ e $\widetilde{\mathrm{V}}_{i}$ a célula de Voronoï associada ao vértice $\mathrm{x}_{i}$ da decomposição dual $\widetilde{\mathcal{V}}=\left\{\mathrm{x}_{i}, \widetilde{\mathrm{V}}_{i}\right\}_{i=1}^{N}$ que satisfaz as condições (2.5b)-(2.5c). Então, existe uma constante $C \in \mathbb{R}_{+}$que independe do parâmetro $h$, tal que

$$
\left|\Delta_{s} u\left(\mathrm{x}_{i}^{c}\right)-\frac{1}{S\left(\widetilde{\mathrm{V}}_{i}\right)} \sum_{j \in \Lambda(i)} m\left(\widetilde{\Gamma}_{i j}\right) \nabla_{s} u\left(\mathrm{~m}_{i j}\right) \cdot \overrightarrow{\mathrm{n}}_{\mathrm{x}, \widetilde{\Gamma}_{i j}}\right| \leq C h,
$$

onde $\mathrm{x}_{i}^{c}$ denota o centroide esférico de $\widetilde{\mathrm{V}}_{i}, m\left(\widetilde{\Gamma}_{i j}\right)$ e $\mathrm{m}_{i j}$ são respectivamente o cumprimento e o ponto médio da aresta $\widetilde{\Gamma}_{i j}$, e $\overrightarrow{\mathrm{n}}_{\mathrm{x}, \widetilde{\Gamma}_{i j}}$ é o vetor normal na aresta $\widetilde{\Gamma}_{i j}$ no ponto $\mathrm{x}$.

Demonstração. Basta tomar as Proposições 3.1 e 3.2 junto à desigualdade triangular.

Resultados da mesma ordem em relação ao tamanho do cumprimento de aresta, foram descritos em diferente contextos por Xu (2006) e Peixoto (2013). Porém, estimativas de ordem dois são possíveis sempre que as células com lados pares satisfaçam um critério de alinhamento de arestas como mostrado por Peixoto e Barros (2013).

Observe-se que, nas Proposições 3.1-3.3 os fluxos da função $u$ através das arestas da célula, são aproximados pelos valores do gradiente tangencial nos pontos médios das arestas. Então, para levar adiante nossa discretização, precisamos construir uma outra aproximação para essas derivadas tangenciais no ponto médio das arestas. Tomaremos esta ideia mediante o seguinte fato: se a derivada normal à célula $\widetilde{\mathrm{V}}_{i}$ em cada aresta $\widetilde{\Gamma}_{i j}$ for aproximada no ponto médio $\mathrm{x}_{i j}$ da aresta esférica triangular $\widetilde{\tau}_{i j}$, teríamos um Método de Diferença Finita centrada (MDF) (centered finite difference) dado como

$$
-\nabla_{s} u\left(\mathrm{x}_{i j}\right) \cdot \overrightarrow{\mathrm{n}}_{\mathrm{x}, \widetilde{\Gamma}_{i j}}=-\frac{u\left(\mathrm{x}_{j}\right)-u\left(\mathrm{x}_{i}\right)}{\left\|\mathrm{x}_{j}-\mathrm{x}_{i}\right\|}+\mathcal{O}\left(h^{2}\right) .
$$

A aproximação da derivada normal acima é simples de implementar e de ordem dois, mas não é ótima, pois estamos aproximando a derivada normal no ponto médio $\mathrm{m}_{i j}$ da aresta dual $\widetilde{\Gamma}_{i j}$. Veja que, se tivéssemos células uniformes na superfície esférica em cada refinamento de malha, os pontos médios $\mathrm{x}_{i j}$ e $\mathrm{m}_{i j}$ das arestas primal e dual coincidiriam. No entanto, como foi discutido por Heikes e Randall (1995b), nas malhas esféricas icosaédricas os pontos médios das arestas primal e dual não coincidem e a sua distância é pelo menos de ordem um, i.e., $\left\|\mathrm{x}_{i j}-\mathrm{m}_{i j}\right\|=\mathcal{O}(h)$. Examinaremos na proposição a seguir, que quando os pontos médios das arestas não coincidem teremos uma aproximação de ordem um para a derivada normal no ponto médio $\mathrm{m}_{i j}$ de $\widetilde{\Gamma}_{i j}$.

Proposição 3.4. Seja $u: \mathbb{S}^{2} \rightarrow \mathbb{R}$ uma função de classe $C^{4}$. Então, a derivada normal no ponto médio $\mathrm{m}_{i j}$ da aresta dual $\widetilde{\Gamma}_{i j}$, que satisfaz a condição (2.5a), pode ser aproximada pelo esquema de diferença finita centrada em termos dos vértices da aresta primal $\widetilde{\tau}_{i j}$. Em outras palavras, existe uma constante $C \in \mathbb{R}_{+}$independente de $h$, tal que

$$
\left|\nabla_{s} u\left(\mathrm{~m}_{i j}\right) \cdot \overrightarrow{\mathrm{n}}_{\mathrm{x}, \widetilde{\Gamma}_{i j}}-\frac{u\left(\mathrm{x}_{j}\right)-u\left(\mathrm{x}_{i}\right)}{\left\|\mathrm{x}_{j}-\mathrm{x}_{i}\right\|}\right| \leq C h,
$$

para $h$ suficientemente pequeno.

Demonstração. Em primeiro lugar, lembremos que as células $\widetilde{\mathrm{V}}_{i}$ e $\widetilde{\mathrm{V}}_{j}$ associadas aos vértices $\mathrm{x}_{i}$ e $\mathrm{x}_{j}$ respectivamente, são adjacentes à aresta $\widetilde{\Gamma}_{i j} \operatorname{com} m\left(\widetilde{\Gamma}_{i j}\right)>0$. Então, de forma análoga à Proposição 3.1, para $u \in C^{4}\left(\mathbb{S}^{2}\right)$, definimos a sua extensão $u^{\Omega}$ a $\Omega_{2}$ e usando o Teorema de Taylor até ordem dois de $u^{\Omega}$ em torno de $\mathrm{m}_{i j}$, temos

$$
u\left(\mathrm{x}_{i}\right)-u\left(\mathrm{~m}_{i j}\right)=u^{\Omega}\left(\mathrm{x}_{i}\right)-u^{\Omega}\left(\mathrm{m}_{i j}\right)=\nabla u^{\Omega}\left(\mathrm{m}_{i j}\right) \cdot\left(\mathrm{x}_{i}-\mathrm{m}_{i j}\right)+R_{i j}^{(i)},
$$


onde $\left|R_{i j}^{(i)}\right| \leq C h^{2} \max _{\mathrm{x} \in \widetilde{\mathrm{V}}_{i}}\left|H\left(u^{\Omega}\right)(\mathrm{x})\right|$. Analogamente,

$$
u\left(\mathrm{x}_{j}\right)-u\left(\mathrm{~m}_{i j}\right)=u^{\Omega}\left(\mathrm{x}_{j}\right)-u^{\Omega}\left(\mathrm{m}_{i j}\right)=\nabla u^{\Omega}\left(\mathrm{m}_{i j}\right) \cdot\left(\mathrm{x}_{j}-\mathrm{m}_{i j}\right)+R_{i j}^{(j)},
$$

$\operatorname{com}\left|R_{i j}^{(j)}\right| \leq C h^{2} \max _{\mathrm{x} \in \widetilde{\mathrm{V}}_{j}}\left|H\left(u^{\Omega}\right)(\mathrm{x})\right|$. Subtraindo (3.5) de (3.6), obteremos

$$
u\left(\mathrm{x}_{j}\right)-u\left(\mathrm{x}_{i}\right)=\nabla u^{\Omega}\left(\mathrm{m}_{i j}\right) \cdot\left(\mathrm{x}_{j}-\mathrm{x}_{i}\right)+R_{i j},
$$

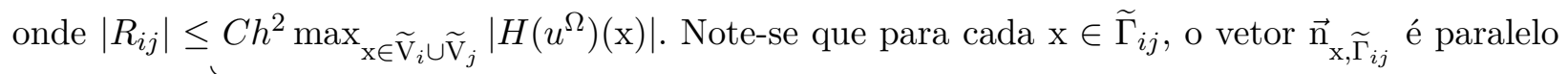
ao vetor $\overrightarrow{\mathrm{x}_{i} \mathrm{x}_{j}}$, devido à bisseção perpendicular entre as arestas conforme enunciada na Subseção 2.2.2, i.e.,

$$
\mathrm{x}_{j}-\mathrm{x}_{i}=\left\|\mathrm{x}_{j}-\mathrm{x}_{i}\right\| \overrightarrow{\mathrm{n}}_{\mathrm{x}, \widetilde{\Gamma}_{i j}}
$$

Então,

$$
u\left(\mathrm{x}_{j}\right)-u\left(\mathrm{x}_{i}\right)=\left\|\mathrm{x}_{j}-\mathrm{x}_{i}\right\| \nabla_{s} u\left(\mathrm{~m}_{i j}\right) \cdot \overrightarrow{\mathrm{n}}_{\mathrm{x}, \widetilde{\Gamma}_{i j}}+R_{i j} .
$$

Por último, invocando a condição (2.5a), o fato $\left\|\mathrm{x}_{j}-\mathrm{x}_{i}\right\| \leq h$, segue que

$$
\left|\frac{u\left(\mathrm{x}_{j}\right)-u\left(\mathrm{x}_{i}\right)}{\left\|\mathrm{x}_{j}-\mathrm{x}_{i}\right\|}-\nabla_{s} u\left(\mathrm{~m}_{i j}\right) \cdot \overrightarrow{\mathrm{n}}_{\mathrm{x}, \widetilde{\Gamma}_{i j}}\right| \leq C h .
$$

O que prova a proposição.

Observação 3.1. Destacamos que na proposição anterior, é possível obter ordem dois em relação ao cumprimento de aresta, se invocarmos o critério de otimização proposto por Heikes e Randall (1995b) para as malhas esféricas icosaédricas e descrito na subseção 2.3.2. A proposta consiste em usar um método de otimização não linear para obter que a diferença entre os pontos médios $\mathrm{x}_{i j}$ e $\mathrm{m}_{i j}$ das arestas primal e dual respectivamente possui ordem dois, i.e., existe uma constante $C \in \mathbb{R}_{+}$, independente de $h$, tal que

$$
\left\|\mathrm{x}_{i j}-\mathrm{m}_{i j}\right\| \leq C h^{2} .
$$

Desta maneira, combinando (3.4) e (3.7) junto à desigualdade triangular, obteremos uma estimativa de ordem dois na Proposição 3.4.

Agora, de forma geral e conforme a Proposição 3.4, temos formalmente que: se $u: \mathbb{S}^{2} \rightarrow \mathbb{R}$ é uma função de classe $C^{4}$, então a derivada normal no ponto médio $\mathrm{m}_{i j}$ da aresta dual $\widetilde{\Gamma}_{i j}$ é aproximada pelo esquema de diferença finita centrada em termos dos vértices da decomposição primal,

$$
-\nabla_{s} u\left(\mathrm{~m}_{i j}\right) \cdot \overrightarrow{\mathrm{n}}_{\mathrm{x}, \widetilde{\Gamma}_{i j}}=-\frac{u\left(\mathrm{x}_{j}\right)-u\left(\mathrm{x}_{i}\right)}{\left\|\mathrm{x}_{j}-\mathrm{x}_{i}\right\|}+\mathcal{O}(h) .
$$

onde $h$ é a medida de malha suficientemente pequena. No que se segue, denotaremos o fluxo discreto da função $u$ através da aresta $\widetilde{\Gamma}_{i j}$ por

$$
\mathrm{F}_{i j}^{d}(u):=-m\left(\widetilde{\Gamma}_{i j}\right) \frac{u\left(\mathrm{x}_{j}\right)-u\left(\mathrm{x}_{i}\right)}{\left\|\mathrm{x}_{j}-\mathrm{x}_{i}\right\|}, \quad \text { para cada } j \in \Lambda(i),
$$

onde $m\left(\widetilde{\Gamma}_{i j}\right)$ é o cumprimento da aresta.

Em vista disso, e como consequência imediata das Proposições 3.1-3.4, podemos enunciar uma expressão do Laplaciano de $u$ no vértice $\mathrm{x}_{i}$ em termos dos fluxos discretos através das arestas da célula $\widetilde{\mathrm{V}}_{i}$ dada como:

$$
-\Delta_{s} u\left(\mathrm{x}_{i}\right) \approx-\frac{1}{S\left(\widetilde{\mathrm{V}}_{i}\right)} \sum_{j \in \Lambda(i)} \mathrm{F}_{i j}^{d}(u) .
$$


Ademais, seja $f_{i}$ o valor médio da função $f$ na célula $\widetilde{\mathrm{V}}_{i}$, i.e.,

$$
f_{i}=\frac{1}{S\left(\widetilde{\mathrm{V}}_{i}\right)} \int_{\widetilde{\mathrm{V}}_{i}} f(\mathrm{x}) d s(\mathrm{x}), \quad \text { para cada } i=1, \ldots, N .
$$

O processo descrito acima permite definir um esquema numérico para a discretização da equação (3.1). Mais precisamente:

Definição 3.1 (Esquema de volumes finitos). Seja $\mathrm{F}_{i j s}^{d}$ o fluxo discreto definido em (3.8), o esquema de volumes finitos para o Laplaciano na esfera unitária é definido pelo sistema:

$$
\left(\mathrm{L}_{s, h}\left(u_{h}\right)\right)_{i}=\frac{1}{S\left(\widetilde{\mathrm{V}}_{i}\right)} \sum_{j \in \Lambda(i)} \mathrm{F}_{i j}^{d}\left(u_{h}\right)=f_{i}, \quad \text { para cada } i=1, \ldots, N,
$$

onde $\mathrm{L}_{s, h}$ denota o operador discreto de Laplace, $u_{h}$ é a solução aproximada, $f_{i}$ é o valor médio da função f em $\widetilde{\mathrm{V}}_{i}$ e $S\left(\widetilde{\mathrm{V}}_{i}\right)$ denota a área superficial da célula.

A expressão (3.10) forma um sistema linear de equações,

$$
\mathrm{L}_{s, h}\left(u_{h}\right)=f_{h},
$$

onde $u_{h}$ é a aproximação constante por pedaços, cujos valores são dados pelo esquema (3.10) e $f_{h}$ é a função constante por pedaços, cujas componentes são os valores $f_{i}$ definidos em (3.9). Vejamos rapidamente uma caracterização da existência da solução discreta. Se a condição de compatibilidade discreta

$$
\left\langle f_{h}, 1\right\rangle_{h}=\sum_{i=1}^{N} S\left(\widetilde{\mathrm{V}}_{i}\right) f_{i}=0,
$$

é satisfeita, implica que o problema discreto (3.11) possui solução. Aqui $\langle\cdot, \cdot\rangle_{h}$ denota o produto escalar discreto. Observe-se que, a expressão acima define uma versão discreta da compatibilidade (1.3) definida na Seção 1.4 para o problema contínuo (1.4). Assim, da aproximação $f_{i}$ temos,

$$
0=\sum_{i=1}^{N} S\left(\widetilde{\mathrm{V}}_{i}\right) f_{i}=\sum_{i=1}^{N} S\left(\widetilde{\mathrm{V}}_{i}\right) \frac{1}{S\left(\widetilde{\mathrm{V}}_{i}\right)} \int_{\widetilde{\mathrm{V}}_{i}} f(\mathrm{x}) d s(\mathrm{x})=\sum_{i=1}^{N} \int_{\widetilde{\mathrm{V}}_{i}} f(\mathrm{x}) d s(\mathrm{x})=\int_{\mathbb{S}^{2}} f(\mathrm{x}) d s(\mathrm{x}),
$$

$\operatorname{logo}$ obtemos $\left\langle f_{h}, 1\right\rangle_{h}=(f, 1)$.

Agora, em relação à unicidade da solução discreta, podemos assumir a condição $\left\langle u_{h}, 1\right\rangle_{h}=0$, mas conforme o objetivo principal desta tese, precisamos definir algumas condições adicionais, as quais serão relatadas em detalhe nas seções posteriores deste capítulo, mais precisamente na Seção 3.3. Por outro lado, no apêndice $\mathrm{C}$ descreve-se brevemente uma caracterização da existência e unicidade da solução do problema discreto via elementos da álgebra linear.

Observação 3.2. O sistema dado em (3.11) pode ser resolvido numericamente usando métodos iterativos clássicos como Jacobi ou Gauss-Seidel, ou também métodos Multigrid como nos artigos de Heikes e Randall (1995b); Heikes et al. (2013). Neste trabalho usamos o método SOR (Succesive Over Relaxation) descrito no Apêndice $C$.

Podemos agora expor um resultado referente ao princípio do mínimo (ou máximo) discreto da solução aproximada.

Lema 3.1. Seja $u_{h}$ a solução discreta não constante de (3.11), tal que $\mathrm{L}_{s, h}\left(u_{h}\right)>0$ (pontualmente). Então, $u_{h}$ não possui mínimo local.

De forma análoga pode-se obter para $u_{h}$ não constante, com $\mathrm{L}_{s, h}\left(u_{h}\right)<0$ (pontualmente), que $u_{h}$ não possui máximo local. 
Demonstração. Suponha que $u_{h}$ atinge seu mínimo no vértice $\mathrm{x}_{i} \in \mathrm{S}_{N}=\left\{\mathrm{x}_{i}\right\}_{i=1}^{N}$. Logo, pela definição do operador discreto segue que,

$$
\left(\mathrm{L}_{s, h}\left(u_{h}\right)\right)_{i}=-\sum_{j \in \Lambda(i)} m\left(\widetilde{\Gamma}_{i j}\right) \frac{u_{h}\left(\mathrm{x}_{j}\right)-u_{h}\left(\mathrm{x}_{i}\right)}{\left\|\mathrm{x}_{j}-\mathrm{x}_{i}\right\|}=\sum_{j \in \Lambda(i)} m\left(\widetilde{\Gamma}_{i j}\right) \frac{u_{h}\left(\mathrm{x}_{i}\right)-u_{h}\left(\mathrm{x}_{j}\right)}{\left\|\mathrm{x}_{j}-\mathrm{x}_{i}\right\|} .
$$

Se $u_{h}\left(\mathrm{x}_{i}\right) \leq u_{h}\left(\mathrm{x}_{j}\right)$ para $j \in \Lambda(i)$, então $\left(\mathrm{L}_{s, h}\left(u_{h}\right)\right)_{i} \leq 0$. Em particular, se $\left(\mathrm{L}_{s, h}\left(u_{h}\right)\right)_{i}=0$, então $u_{h}\left(\mathrm{x}_{i}\right)=u_{h}\left(\mathrm{x}_{j}\right)$ para todo vértice $\mathrm{x}_{j} \in \mathrm{S}_{N}=\left\{\mathrm{x}_{i}\right\}_{i=1}^{N}$, e em consequência $u_{h}$ seria constante na esfera toda, uma contradição. Portanto $u_{h}$ não possui mínimo local.

Dado que o MVF discretiza a forma integral (3.1) direitamente, uma virtude é a propriedade de conservação. Logo, o esquema (3.10) é naturalmente conservativo, se duas células $\widetilde{\mathrm{V}}_{i} \mathrm{e} \widetilde{\mathrm{V}}_{j}$ adjacentes à aresta $\widetilde{\Gamma}_{i j} \operatorname{com} m\left(\widetilde{\Gamma}_{i j}\right)>0$, logo a aproximação $\mathrm{F}_{i j}^{d}$ em $\widetilde{\mathrm{V}}_{i}$ é igual a $-\mathrm{F}_{j i}^{d}$ dada em $\widetilde{\mathrm{V}}_{j}$. Definimos, então a propriedade:

Definição 3.2 (Conservação discreta). Sejam uh a única solução ao sistema (3.11) e $\mathrm{F}_{i j}^{d}$ o fluxo discreto definido em (3.8). O esquema de volumes finitos (3.10) é dito conservativo, se satisfaz da condição

$$
\sum_{i=1}^{N} \sum_{j \in \Lambda(i)} \mathrm{F}_{i j}^{d}\left(u_{h}\right)=0
$$

o qual segue de $\mathrm{F}_{i j}^{d}=-\mathrm{F}_{j i}^{d}$, para $\mathrm{x}_{i}$ e $\mathrm{x}_{j}$ vizinhos adjacentes com $m\left(\widetilde{\Gamma}_{i j}\right)>0$.

\subsection{Erro de truncamento}

Nesta seção discutiremos brevemente alguns fatos inerentes à discretização do Laplaciano, especificamente o erro da consistência, que no entanto não interferem nas nossas provas de convergência das soluções. Lembrando que para estudar a convergência das soluções dos MVF, podemos optar por dois caminhos distintos: o primeiro é assumir o MVF como um MDF, dado que o fluxo na fronteira da célula é aproximado por uma diferença finita centrada, como definido em (3.10) e é o mais usado nas decomposições Voronoï-Delaunay devido à bisseção perpendicular. O segundo caminho, é escrever o MVF como um Método de Elementos Finitos (MEF) baseado na formulação variacional de (1.4). Esta última opção será executada nesta tese e detalhada nas seções posteriores para obter a estimações da estabilidade e da convergência das soluções do MVF.

Dado que (3.11) usa um esquema de diferença finita vamos usar a terminologia da análise de convergência dos MDF. O erro de truncamento ou consistência é obtido aplicando o operador discreto $\mathrm{L}_{s, h}(\cdot)$ à solução exata $u: \mathbb{S}^{2} \rightarrow \mathbb{R}$,

$$
\xi_{h}:=\mathrm{L}_{s, h}(\widetilde{u})-\mathrm{L}_{s, h}\left(u_{h}\right)=\mathrm{L}_{s, h}(\widetilde{u})-f_{h},
$$

onde $\widetilde{u}$ representa os valores exatos de $u$ em cada vértice de $\mathrm{S}_{N}=\left\{\mathrm{x}_{i}\right\}_{i=1}^{N}$. Note-se que o erro $\xi_{h}$ é a função constante por pedaços, cujas componentes são definidas por

$$
\xi_{i}=\left(\mathrm{L}_{s, h}(\widetilde{u})\right)_{i}-f_{i}=\left(\mathrm{L}_{s, h}(\widetilde{u})\right)_{i}-\frac{1}{S\left(\widetilde{\mathrm{V}}_{i}\right)} \sum_{j \in \Lambda(i)} \mathrm{F}_{i j}^{d}(u), \quad \text { para } i=1, \ldots, N .
$$

A equação (3.13) é de grande interesse, pois dela poderíamos estimar a diferença entre $\widetilde{u}$ e a sua aproximação $u_{h}$ em termos do erro de consistência (Teorema de Lax-Richtmyer, vide por exemplo Strikwerda, 2004, pp. 32). Não obstante, o esquema (3.11), não fornece uma aproximação consistente do Laplaciano. Em princípio, para um tamanho de malha $h$ indo para zero e combinando as Proposições 3.3 e 3.4, obtemos que a medida da aproximação do Laplaciano é assintoticamente inconsistente, i.e., o erro de truncamento não vai para zero, quando incrementamos o refinamento da malha. Frequentemente, a perda dessa ordem de precisão é causada pela aproximação da integral de linha na aresta e após pela aproximação da derivada normal pelo esquema das diferenças finitas centradas no ponto médio da aresta dual (Heikes e Randall, 1995b; Ringler, 2003). 
Apesar disso, esse problema de inconsistência na aproximação pode ser corrigido incorporando uma condição alternativa conhecida na literatura como consistência fraca (Coudière et al., 1999; Faille, 1992), a qual consiste em assumir que os fluxos contínuos possuem aproximações consistentes nas arestas (vide Apêndice A). Então, tendo consistência fraca aliada com hipóteses suficientes de estabilidade poderia-se garantir a convergência do esquema no sentido das diferenças finitas. Para contextualizar esse fato, apresentamos o resultado relatado por Du et al. (2003b, Proposição 3, pp. 3943), o qual faz uma extensão a $\mathbb{S}^{2}$ da análise feita por Gallouët et al. (2000); Mishev (1998) e Eymard et al. (2001) para a $L^{2}$-norma. A prova da seguinte proposição será detalhada no Apêndice A.

Proposição 3.5 (Du et al., 2003b). Assuma $\widetilde{\mathcal{V}}=\left\{\mathrm{x}_{i}, \widetilde{\mathrm{V}}_{i}\right\}_{i=1}^{N}$ a decomposição dual de Voronoï de $\mathbb{S}^{2}, u \in H^{2}\left(\mathbb{S}^{2}\right) \cap H_{Q}^{1}\left(\mathbb{S}^{2}\right)$ é a única solução ao problema (1.4) e considere o esquema dos volumes finitos definido em (3.11). Suponha também que as condições de ortogonalidade entre as arestas primal/dual e que o fluxo $\mathrm{F}_{i j}^{c}$ possui aproximações consistentes. Então, o erro de truncamento atende a estimativa

$$
\left\|\widetilde{\xi}_{h}\right\|_{0, h} \leq C h\|u\|_{H^{2}\left(\mathbb{S}^{2}\right)},
$$

onde $C \in \mathbb{R}_{+}$é uma constante que depende apenas da medida de regularidade da malha $\sigma$ definida em (2.2).

Um segundo resultado tem a abordagem do artigo de Faille (1992) para um problema de Laplace planar em malhas retangulares ou paralelepipédicas na max-norma. Estendemos a prova a $\mathbb{S}^{2}$ incorporando as condições $(2.5 \mathrm{a})-(2.5 \mathrm{c})$ obtendo que o operador discreto satisfaz a condição de consistência fraca:

Proposição 3.6. Assuma $\widetilde{\mathcal{V}}=\left\{\mathrm{x}_{i}, \widetilde{\mathrm{V}}_{i}\right\}_{i=1}^{N}$ a decomposição dual de Voronö̈ de $\mathbb{S}^{2}$ que satisfaz as condições (2.5a)-(2.5c), $u \in H^{2}\left(\mathbb{S}^{2}\right) \cap H_{Q}^{1}\left(\mathbb{S}^{2}\right)$ a única solução ao problema (1.4). Considere que o esquema (3.11) é conservativo. Suponha também que o fluxo $\mathrm{F}_{i j}^{c}$ possui aproximações consistentes. Então, o erro de truncamento converge para zero na topologia fraca $\star$, i.e., atende o critério de dualidade,

$$
\int_{\mathbb{S}^{2}} \widetilde{\xi}_{h}(\mathrm{x}) v(\mathrm{x}) d s(\mathrm{x}) \longrightarrow 0, \quad \text { quando } h \longrightarrow 0, \text { para cada } v \in L^{1}\left(\mathbb{S}^{2}\right) .
$$

O esquema é dito que satisfaz a propriedade de consistência fraca.

Porém, algumas hipóteses da estabilidade não podem ser assumidas de forma simples e certos cuidados são necessários. Especificamente, o esquema (3.11) não satisfaz o principio do máximo discreto, Lema 3.1, e ademais a matriz associada ao operador discreto é singular (Hackbusch, 2017, Teorema 4.6.2, pp. 77). Nesse sentido, uma prova sobre a ordem de convergência na max-norma não é atendida diretamente da ordem de consistência fraca (Pascal, 2007).

\subsubsection{Experimentos numéricos}

Nesta subseção, mostraremos um experimento numérico sobre a discretização do Laplaciano usando o esquema (3.11). Sejam $u$ e $f$ funções contínuas dadas em coordenadas geográficas latitudelongitude $(\phi, \theta)$ (vide Apêndice B). Consideraremos o exemplo de Du e Ju (2005) cuja solução exata é

$$
u(\phi, \theta)=\exp (\sin (\phi))
$$

e o respectivo Laplaciano é definido como

$$
f(\phi, \theta)=\exp (\sin (\phi))\left(\cos ^{2}(\phi)-2 \sin (\phi)\right) .
$$

Consideraremos também a malha esférica icosaédrica NOPT e as duas otimizações SCVT e HR95 definidas recursivamente como no Capítulo 2. Em particular, para o caso da SCVT usaremos uma malha uniformemente distribuída com função de densidade constante $\rho(\mathrm{x})=1$ para cada $\mathrm{x} \in \mathbb{S}^{2}$. A fim de verificar o erro de truncamento $\xi_{h}$, usaremos o erro quadrático médio e o máximo, definidos 


\begin{tabular}{|c|c|c|c|c|c|c|}
\hline \multicolumn{7}{|c|}{ ERRO DE TRUNCAMENTO E TAXA DE CONVERGÊNCIA $p$} \\
\hline & \multicolumn{2}{|c|}{ NOPT } & \multicolumn{2}{|c|}{ SCVT } & \multicolumn{2}{|c|}{ HR95 } \\
\hline NÍVEL & $E_{2}\left(\xi_{h}\right)$ & $p$ & $E_{2}\left(\xi_{h}\right)$ & $p$ & $E_{2}\left(\xi_{h}\right)$ & $p$ \\
\hline 1 & $1.445 \mathrm{E}-01$ & - & $1.445 \mathrm{E}-01$ & - & $1.445 \mathrm{E}-01$ & - \\
\hline 2 & 4.612E-02 & $1.648 \mathrm{E}+00$ & $3.923 \mathrm{E}-02$ & $1.881 \mathrm{E}+00$ & $3.828 \mathrm{E}-02$ & $1.917 \mathrm{E}+00$ \\
\hline 3 & $1.575 \mathrm{E}-02$ & $1.550 \mathrm{E}+00$ & $1.071 \mathrm{E}-02$ & $1.873 \mathrm{E}+00$ & $1.034 \mathrm{E}-02$ & $1.888 \mathrm{E}+00$ \\
\hline 4 & $6.115 \mathrm{E}-03$ & $1.365 \mathrm{E}+00$ & $3.299 \mathrm{E}-03$ & $1.699 \mathrm{E}+00$ & $2.880 \mathrm{E}-03$ & $1.845 \mathrm{E}+00$ \\
\hline 5 & $2.658 \mathrm{E}-03$ & $1.202 \mathrm{E}+00$ & $1.257 \mathrm{E}-03$ & $1.392 \mathrm{E}+00$ & $8.423 \mathrm{E}-04$ & $1.774 \mathrm{E}+00$ \\
\hline 6 & $1.238 \mathrm{E}-03$ & $1.102 \mathrm{E}+00$ & $5.670 \mathrm{E}-04$ & $1.148 \mathrm{E}+00$ & 2.611E-04 & $1.690 \mathrm{E}+00$ \\
\hline 7 & $5.976 \mathrm{E}-04$ & $1.050 \mathrm{E}+00$ & $2.749 \mathrm{E}-04$ & $1.044 \mathrm{E}+00$ & $8.618 \mathrm{E}-05$ & $1.599 \mathrm{E}+00$ \\
\hline 8 & 2.937E-04 & $1.025 \mathrm{E}+00$ & $1.362 \mathrm{E}-04$ & $1.013 \mathrm{E}+00$ & $2.936 \mathrm{E}-05$ & $1.554 \mathrm{E}+00$ \\
\hline & $E_{\infty}\left(\xi_{h}\right)$ & $p$ & $E_{\infty}\left(\xi_{h}\right)$ & $p$ & $E_{\infty}\left(\xi_{h}\right)$ & $p$ \\
\hline 1 & $4.982 \mathrm{E}-01$ & - & $4.982 \mathrm{E}-01$ & - & $4.982 \mathrm{E}-01$ & - \\
\hline 2 & $1.416 \mathrm{E}-01$ & $1.814 \mathrm{E}+00$ & $1.283 \mathrm{E}-01$ & $1.957 \mathrm{E}+00$ & $1.379 \mathrm{E}-01$ & $1.853 \mathrm{E}+00$ \\
\hline 3 & $7.896 \mathrm{E}-02$ & $8.430 \mathrm{E}-01$ & 3.445E-02 & $1.898 \mathrm{E}+00$ & $3.596 \mathrm{E}-02$ & $1.939 \mathrm{E}+00$ \\
\hline 4 & 7.328E-02 & $1.077 \mathrm{E}-01$ & $2.314 \mathrm{E}-02$ & $5.737 \mathrm{E}-01$ & $1.205 \mathrm{E}-02$ & $1.578 \mathrm{E}+00$ \\
\hline 5 & 7.184E-02 & $2.849 \mathrm{E}-02$ & 2.403E-02 & $5.430 \mathrm{E}-02$ & $5.839 \mathrm{E}-03$ & $1.045 \mathrm{E}+00$ \\
\hline 6 & 7.149E-02 & 7.227E-03 & $2.427 \mathrm{E}-02$ & $1.428 \mathrm{E}-02$ & 2.911E-03 & $1.004 \mathrm{E}+00$ \\
\hline 7 & $7.140 \mathrm{E}-02$ & $1.813 \mathrm{E}-03$ & $2.433 \mathrm{E}-02$ & $3.500 \mathrm{E}-03$ & $1.491 \mathrm{E}-03$ & $9.653 \mathrm{E}-01$ \\
\hline 8 & $7.137 \mathrm{E}-02$ & $4.537 \mathrm{E}-04$ & $2.434 \mathrm{E}-02$ & $7.036 \mathrm{E}-04$ & 7.377E-04 & $1.015 \mathrm{E}+00$ \\
\hline
\end{tabular}

Tabela 3.1: Estimativas do erro de truncamento $\xi_{h}$ nas $E_{2}$ e $E_{\infty}$-normas e a taxa de convergência $p$ do Laplaciano discreto (3.10) para a função (3.14) nas malhas NOPT, SCVT e HR95 com níveis de refinamento de 1 até 8.

respectivamente como

$$
E_{2}\left(\xi_{h}\right)=\sqrt{\frac{1}{N} \sum_{i=1}^{N} \xi_{i}^{2}}, \quad E_{\infty}\left(\xi_{h}\right)=\max _{i=1, \ldots, N}\left|\xi_{i}\right|
$$

A Tabela 3.1 expõe os valores do erro de truncamento $\xi_{h}$ e a sua respectiva taxa de convergência $p$ do esquema discreto para o exemplo (3.15) com as $E_{2}$ e $E_{\infty}$-normas. Observe-se que a taxa de convergência $p$ nas malhas NOPT e SCVT é próximo de um para a $E_{2}$-norma, o que confirmaria a Proposição 3.5. Por outro lado, Note-se que nessas malhas o erro de truncamento $\xi_{h}$ cessa de cair a partir do nível de refinamento 4 na $E_{\infty}$-norma. Mostrando que o operador é assintoticamente inconsistente ao aumentar o nível de refinamento. Por sua vez, a malha HR95 mostra uma taxa de convergência $p$ entre um e dois na $E_{2}$-norma e próxima de um na $E_{\infty}$-norma. Esse comportamento é devido ao critério geométrico da otimização conforme relatado por Heikes e Randall (1995b).

A Figura 3.2 representa graficamente os valores dados na Tabela 3.1. O eixo horizontal representa o nível de refinamento $\ell$ de 1 até 8 . No eixo vertical estão representados os erros de truncamento nas duas normas e a sua respectiva taxa de convergência $p$ para as malhas NOPT (triângulo azul), SCVT (diamante vermelho) e HR95 (quadrado verde).

\subsection{Relação MVF-MEFL e estabilidade}

Nesta seção estaremos interessados no estudo da estabilidade de nosso esquema (3.11). A abordagem será feita conforme a literatura (Li et al., 2000; Zhang, 2014), considerando o MVF como um MEFL. Em outras palavras, a aproximação construída pelo MVF será uma perturbação da aproximação dos MEFL. Logo, escrevendo (3.11) em uma forma variacional adequada, estimaremos a estabilidade das soluções aproximadas. 

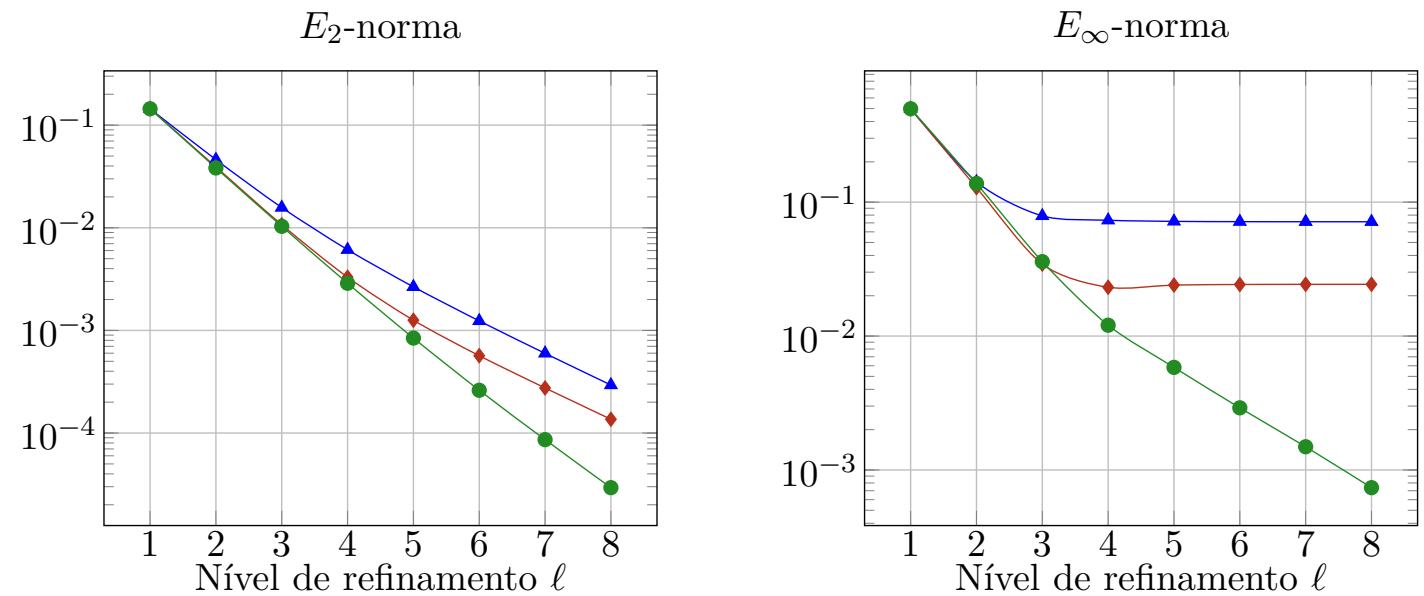

$\neg \mathrm{NOPT} \rightarrow \mathrm{SCVT} \bullet \mathrm{HR} 95$

Figura 3.2: Erro de truncamento $\xi_{h}$ e a sua respectiva taxa de convergência $p$ do Laplaciano discreto da função (3.14) nas $E_{2}$ e $E_{\infty}$-norma com as malhas: NOPT (triângulo azul), SCVT (diamante vermelho) $e$ HR95 (quadrado verde) respectivamente nos niveis de refinamento de malha 1 (42 vértices) até 8 (655362 vértices).

\subsubsection{Correspondência geométrica}

Começamos incorporando alguns argumentos geométricos dos trabalhos de Demlow e Dziuk (2007); Du e Ju (2005); Dziuk e Elliott (2013); Ju e Du (2009) e Ju et al. (2009) para as decomposições Voronoï-Delaunay em $\mathbb{S}^{2}$. Assumamos que $\mathbb{S}^{2}$ é aproximada por uma sequencia de poliedros $\mathbf{S}_{h}$ formada pela decomposição $\mathcal{T}_{h}$ (triângulos planos) com $h$ indo para zero. Também assumamos, por construção, que para cada $\mathrm{x} \in \mathbb{S}^{2}$, há no máximo um ponto $\mathrm{x}^{*} \in \mathbf{S}_{h}$ tal que $\mathrm{x}=\frac{\mathrm{x}^{*}}{\left\|\mathrm{x}^{*}\right\|}$ e que os vértices $\mathrm{S}_{N}=\left\{\mathrm{x}_{i}\right\}_{i=1}^{N}$ de $\mathbf{S}_{h}$ estejam na superfície de $\mathbb{S}^{2}$, tal que $\mathrm{S}_{N}:=\mathbf{S}_{h} \cap \mathbb{S}^{2}$. Desta maneira, temos que

$$
\mathbf{S}_{h}=\bigcup_{\mathrm{T}_{i j k} \in \mathcal{T}_{h}} \operatorname{cl}\left(\mathrm{T}_{i j k}\right), \quad \mathbb{S}^{2}=\underset{\widetilde{\mathrm{T}}_{i j k} \in \widetilde{\mathcal{T}}_{h}}{\bigcup} \mathrm{cl}\left(\widetilde{\mathrm{T}}_{i j k}\right)
$$

onde $\operatorname{cl}(\cdot)$ denota o fecho e $\mathrm{T}_{i j k}$ e $\widetilde{\mathrm{T}}_{i j k}$ denotam os triângulos plano e esférico com vértices $\mathrm{x}_{i}, \mathrm{x}_{j}$ e $\mathrm{x}_{k}$. Deve-se notar que o poliedro de nível zero ou 0-glevel é o icosaedro puro. Consideraremos o seguinte definição:

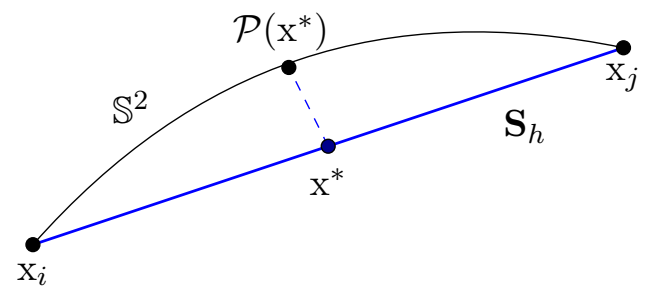

Figura 3.3: Projeção radial do poliedro $\mathbf{S}_{h}$ na esfera $\mathbb{S}^{2}$.

Definição 3.3. (Projeção radial) Seja $\mathrm{x}^{*} \in \mathbb{R}^{3} \backslash\{0\}$ um ponto no poliedro de aproximação $\mathbf{S}_{h} . A$ aplicação $\mathcal{P}: \mathbf{S}_{h} \rightarrow \mathbb{S}^{2}$ suave e bijectiva, é definida como a projeção radial do ponto $\mathrm{x}^{*}$ na superfície esférica, i.e., $\mathcal{P}\left(\mathrm{x}^{*}\right)=\mathrm{x}^{*} /\left\|\mathrm{x}^{*}\right\|$ (Figura 3.3).

A seguinte proposição, dada por Du e Ju (2005, Seção 3, pp. 1677), define uma aproximação da geometria através da projeção radial. 
Proposição 3.7 (Du e Ju, 2005). Sejam $\mathbb{S}^{2}$ a esfera unitária e $\mathbf{S}_{h}$ o seu poliedro de aproximação. Para qualquer $\mathrm{x} \in \mathbb{S}^{2}$, e para cada $\widetilde{\mathrm{T}}_{i j k} \subset \mathbb{S}^{2}$ e $\mathrm{T}_{i j k} \subset \mathbf{S}_{h}$, existem constantes $C \in \mathbb{R}_{+}$tal que

$$
\left\|\mathrm{x}-\mathcal{P}^{-1}(\mathrm{x})\right\| \leq C h^{2}, \quad S\left(\mathrm{~T}_{i j k}\right) \leq S\left(\widetilde{\mathrm{T}}_{i j k}\right) \leq\left(1+C h^{2}\right) S\left(\mathrm{~T}_{i j k}\right) .
$$

Para levar adiante nosso estudo, consideraremos o conjunto $\Omega_{h} \subset \mathbb{R}^{3}$, dado por

$$
\Omega_{h}:=\left\{\mathrm{x}^{*} \in \mathbb{R}^{3} \backslash\{0\}: 1-C h^{2}<\left\|\mathrm{x}^{*}\right\|<1+C h^{2}\right\},
$$

onde $C \in \mathbb{R}_{+}$é uma constante independente de $h$. Note-se que tanto $\mathbb{S}^{2}$ como $\mathbf{S}_{h}$ são subconjuntos de $\Omega_{h}$. No que se segue, para cada função $u \in H^{2}\left(\mathbb{S}^{2}\right)$, denotamos por $u^{\Omega}$ a extensão de $u$ a $\Omega_{h}$, tal que $u^{\Omega}\left(\mathrm{x}^{*}\right)=u\left(\mathcal{P}\left(\mathrm{x}^{*}\right)\right)$ para cada $\mathrm{x}^{*} \in \Omega_{h}$. O seguinte resultado foi mostrado por Du et al. (2003b, Lema 1, pp. 3941).

Proposição 3.8 (Du et al., 2003b). Seja $u \in H^{2}\left(\mathbb{S}^{2}\right)$. Para qualquer $\mathrm{x}^{*} \in \Omega_{h} \operatorname{com} \mathrm{x}=\mathcal{P}\left(\mathrm{x}^{*}\right) \in \mathbb{S}^{2}$, segue que

$$
\begin{aligned}
\nabla_{s} u(\mathrm{x}) & =\nabla u^{\Omega}(\mathrm{x}), \quad \nabla\left(\partial_{n} u^{\Omega}(\mathrm{x})\right)=\nabla_{s}\left(\partial_{s, n} u\right)(\mathrm{x})-\left(\partial_{s, n} u(\mathrm{x})\right) \overrightarrow{\mathrm{n}}_{\mathbb{S}^{2}, \mathrm{x}}, \\
\left\|\mathrm{x}^{*}\right\| \nabla u^{\Omega}\left(\mathrm{x}^{*}\right) & =\nabla u^{\Omega}(\mathrm{x}), \quad\left\|\mathrm{x}^{*}\right\|^{2} \partial_{n} \partial_{m} u^{\Omega}\left(\mathrm{x}^{*}\right)=\partial_{n} \partial_{m} u^{\Omega}(\mathrm{x}),
\end{aligned}
$$

onde $\partial_{n}$ e $\partial_{s, n}$ denotam a derivada parcial e tangencial respectivamente na coordenada $n$ do ponto $\mathrm{x} \in \mathbb{S}^{2}$. A seguinte proposição compara as normas de funções definidas tanto em $\mathbb{S}^{2}$ como em $\mathbf{S}_{h}$. A prova está contida nos artigos de Dziuk e Elliott (2013, Lema 4.2, pp 312) e Demlow (2009, pp, 811). No entanto, reproduzimos uma parte da prova para colocá-la no nosso contexto.

Proposição 3.9 (Demlow, 2009). Para cada $u \in H^{2}\left(\mathbb{S}^{2}\right) \cap H_{Q}^{1}\left(\mathbb{S}^{2}\right)$ e qualquer par de triângulos esférico $\widetilde{\mathrm{T}}_{i j k} \in \widetilde{\mathcal{T}}_{h}$ e plano $\mathrm{T}_{i j k} \in \mathcal{T}_{h}$, existem constantes $C_{n} \in \mathbb{R}_{+}$, com $n \in\{0,1,2\}$, independentes de $h$, tal que

$$
\begin{aligned}
\frac{1}{C_{0}}\|u\|_{L^{2}\left(\widetilde{\mathrm{T}}_{i j k}\right)} & \leq\left\|\bar{u}^{\Omega}\right\|_{L^{2}\left(\mathrm{~T}_{i j k}\right)} \leq C_{0}\|u\|_{L^{2}\left(\widetilde{\mathrm{T}}_{i j k}\right)}, \\
\frac{1}{C_{1}}\|u\|_{H^{1}\left(\widetilde{\mathrm{T}}_{i j k}\right)} & \leq\left\|\bar{u}^{\Omega}\right\|_{H^{1}\left(\mathrm{~T}_{i j k}\right)} \leq C_{1}\|u\|_{H^{1}\left(\widetilde{\mathrm{T}}_{i j k}\right)}, \\
\left\|\bar{u}^{\Omega}\right\|_{H^{2}\left(\mathrm{~T}_{i j k}\right)} & \leq C_{2}\|u\|_{H^{2}\left(\widetilde{\mathrm{T}}_{i j k}\right)},
\end{aligned}
$$

onde $\bar{u}^{\Omega}$ denota a extensão da função u a $\Omega_{h}$ restrita ao poliedro $\mathbf{S}_{h}$, i.e., $\bar{u}^{\Omega}=\left.u^{\Omega}\right|_{\mathbf{S}_{h}}$.

Demonstração. Para (3.16a), seja $\bar{u}^{\Omega}$ a extensão de $u$ a $\Omega_{h}$ restrita a $\mathbf{S}_{h}$. Com efeito, se $\mathrm{x}=\mathcal{P}\left(\mathrm{x}^{*}\right) \in$ $\mathbb{S}^{2}$ para cada $\mathrm{x}^{*} \in \mathrm{T}_{i j k}$, segue que $u(\mathrm{x})=u^{\Omega}\left(\mathrm{x}^{*}\right)$. Assim,

$$
\|u\|_{L^{2}\left(\widetilde{\mathrm{T}}_{i j k}\right)}^{2}=\int_{\widetilde{\mathrm{T}}_{i j k}}|u(\mathrm{x})|^{2} d s(\mathrm{x})=\int_{\widetilde{\mathrm{T}}_{i j k}}\left|u^{\Omega}\left(\mathcal{P}^{-1}(\mathrm{x})\right)\right|^{2} d s(\mathrm{x}) .
$$

Pela mudança de variável onde $d s(\mathrm{x})=\frac{1}{\left\|\mathrm{x}^{*}\right\|} d s\left(\mathrm{x}^{*}\right)$, com $0<\frac{1}{C} \leq \frac{1}{\left\|\mathrm{x}^{*}\right\|} \leq C<\infty$ (Dziuk e Elliott, 2013, Lema 1, pp 310), temos que,

$$
\int_{\widetilde{\mathrm{T}}_{i j k}}\left|u^{\Omega}\left(\mathcal{P}^{-1}(\mathrm{x})\right)\right|^{2} d s(\mathrm{x})=\int_{\mathrm{T}_{i j k}}\left|\bar{u}^{\Omega}\left(\mathrm{x}^{*}\right)\right|^{2} \frac{1}{\left\|\mathrm{x}^{*}\right\|} d s\left(\mathrm{x}^{*}\right) \leq C \int_{\mathrm{T}_{i j k}}\left|\bar{u}^{\Omega}\left(\mathrm{x}^{*}\right)\right|^{2} d s\left(\mathrm{x}^{*}\right) .
$$

O que implicaria,

$$
\|u\|_{L^{2}\left(\widetilde{T}_{i j k}\right)} \leq C_{0}\left\|\bar{u}^{\Omega}\right\|_{L^{2}\left(\mathrm{~T}_{i j k}\right)},
$$

Analogamente, podemos deduzir o lado direito de (3.16a). Agora, para (3.16b), pela mudança de 
variável e a Proposição 3.8, obtemos

$$
\left\|\nabla_{s} u\right\|_{L^{2}\left(\widetilde{\mathrm{T}}_{i j k}\right)}^{2}=\int_{\widetilde{\mathrm{T}}_{i j k}}\left|\nabla_{s} u(\mathrm{x})\right|^{2} d s(\mathrm{x}) \leq C \int_{\mathrm{T}_{i j k}}\left|\nabla \bar{u}^{\Omega}\left(\mathrm{x}^{*}\right)\right|^{2} d s\left(\mathrm{x}^{*}\right) .
$$

Pela estimativa (3.16a) junto a desigualdade de Poincaré-Friedrich, Proposição 1.1, segue para alguma constante $C_{1} \in \mathbb{R}_{+}$que

$$
\|u\|_{H^{1}\left(\widetilde{\mathrm{T}}_{i j k}\right)} \leq C_{1}\left\|\bar{u}^{\Omega}\right\|_{H^{1}\left(\mathrm{~T}_{i j k}\right)} .
$$

O lado direito de (3.16b) e (3.16c) são deduzidas de maneira similar.

Observação 3.3. A proposição anterior compara as normas de Sobolev das funções definidas tanto em $\mathbb{S}^{2}$ como em $\mathbf{S}_{h}$. Também pode ser enfraquecida para funções $u \in W^{k, p}\left(\mathbb{S}^{2}\right)$ com $k \geq 0$ e $1 \leq p \leq \infty$. Então, existiram constantes $C_{k} \in \mathbb{R}_{+}$que dependerão só de $k$ e $\mathbb{S}^{2}$.

\subsubsection{Espaços de aproximação}

Vamos introduzir o relacionamento dos MVF com os Métodos dos Elementos Finitos Lineares (MEFL). Para fim, conforme Dziuk e Elliott (2013); Ju e Du (2009) e Ju et al. (2009), configuraremos o MEFL no poliedro $\mathbf{S}_{h}$, como sendo que a decomposição $\mathcal{T}_{h}$, conformada pelos triângulos planos, é uma malha quase-uniforme (no sentido das condições (2.5b)-(2.5c)). Desta maneira, o espaço dos elementos finitos em $\mathbf{S}_{h}$ é definido como

$$
\mathbb{P}_{1}\left(\mathbf{S}_{h}, \mathcal{T}_{h}\right):=\left\{\bar{u}_{h} \in C\left(\mathbf{S}_{h}\right):\left.\bar{u}_{h}\right|_{\mathrm{T}_{i j k}} \text { é linear para cada } \mathrm{T}_{i j k} \in \mathcal{T}_{h}\right\} .
$$

Ademais é gerado pelas funções base nodais $\bar{\varphi}_{1, h}, \ldots, \bar{\varphi}_{N, h}$, definidas por

$$
\bar{\varphi}_{i, h}\left(\mathrm{x}_{j}\right)=\delta_{i j}, \quad \text { para } i=1, \ldots, N,
$$

onde $\mathrm{x}_{j}$ são os vértices em $\mathrm{S}_{N}=\left\{\mathrm{x}_{i}\right\}_{i=1}^{N}$ e $\delta_{i j}$ denota o delta de Kronecker. Ademais, note-se que a dimensão de $U_{h}$ é $\operatorname{Dim}\left(U_{h}\right)=N$. Toda função $\bar{u}_{h} \in \mathbb{P}_{1}\left(\mathbf{S}_{h}, \mathcal{T}_{h}\right)$ tem a forma

$$
\bar{u}_{h}\left(\mathrm{x}^{*}\right)=\sum_{i=1}^{N} \alpha_{i} \bar{\varphi}_{i, h}\left(\mathrm{x}^{*}\right), \quad \text { para cada } \mathrm{x}^{*} \in \mathbf{S}_{h},
$$

com constantes reais $\alpha_{i}(i=1, \ldots, N)$. Observe-se que a extensão $\bar{u}_{h}^{\Omega}$ de $\bar{u}_{h}$ a $\Omega_{h}$ satisfaz $\bar{u}_{h}^{\Omega}\left(\mathrm{x}^{*}\right)=\bar{u}_{h}\left(\mathrm{x}^{*}\right)$ para cada $\mathrm{x}^{*} \in \mathbf{S}_{h}$. Desta maneira, podemos considerar o espaço de Sobolev em $\mathbf{S}_{h}$, no sentido de funções lineares por pedaços. Em virtude da correspondência geométrica entre $\mathbb{S}^{2}$ e $\mathbf{S}_{h}$, podemos estabelecer o espaço de elementos finitos associado à decomposição primal $\widetilde{\mathcal{T}}_{h}$ dado como,

$$
\mathbb{P}_{1}\left(\mathbb{S}^{2}, \widetilde{\mathcal{T}}_{h}\right):=\left\{u_{h} \in C\left(\mathbb{S}^{2}\right): u_{h}=\bar{u}_{h} \circ \mathcal{P}^{-1}, \quad \text { para cada } \bar{u}_{h} \in \mathbb{P}_{1}\left(\mathbf{S}_{h}, \mathcal{T}_{h}\right)\right\}
$$

onde $\mathcal{P}$ denota a projeção radial. Vejamos que $\mathbb{P}_{1}\left(\mathbb{S}^{2}, \widetilde{\mathcal{T}}_{h}\right) \subset H^{1}\left(\mathbb{S}^{2}\right)$, com efeito, se consideramos $u_{h} \in \mathbb{P}_{1}\left(\mathbb{S}^{2}, \widetilde{\mathcal{T}}_{h}\right)$, munido com a $H^{1}$-norma e o fato $\left\{\bar{u}_{h}\left(\mathrm{x}_{i}\right)=u_{h}\left(\mathrm{x}_{i}\right)\right\}_{i=1}^{N}$, obteremos da Proposição 3.8 , que $\bar{u}_{h}^{\Omega}=\bar{u}_{h} \in H^{1}\left(\mathbf{S}_{h}\right)$ e em consequência $u_{h} \in H^{1}\left(\mathbb{S}^{2}\right)$. Para mais detalhes vide Demlow (2009) ou Demlow e Dziuk (2007). Agora, precisamos aproximar funções do espaço quociente $H_{Q}^{1}\left(\mathbb{S}^{2}\right)$. Então, consideremos um subespaço de $\mathbb{P}_{1}\left(\mathbb{S}^{2}, \widetilde{\mathcal{T}}_{h}\right)$ dado como

$$
\widetilde{U}_{h}\left(\widetilde{\mathcal{T}}_{h}\right):=\left\{u_{h} \in \mathbb{P}_{1}\left(\mathbb{S}^{2}, \widetilde{\mathcal{T}}_{h}\right): \int_{\mathbb{S}^{2}} u_{h}(\mathrm{x}) d s(\mathrm{x})=0\right\}
$$

i.e., o espaço de funções $u_{h} \in \mathbb{P}_{1}\left(\mathbb{S}^{2}, \widetilde{\mathcal{T}}_{h}\right)$ que possuem média zero.

Conforme a literatura (vide por exemplo Li et al., 2000 ou Lin et al., 2013), a construção de $\widetilde{U}_{h}$ permite de algum modo, determinar um espaço adequado de funções associadas à decomposição 
dual de Voronoï $\widetilde{\mathcal{V}}=\left\{\mathrm{x}_{i}, \widetilde{\mathrm{V}}_{i}\right\}_{i=1}^{N}$, cujos elementos são polinômios de menor grau que os de $\widetilde{U}_{h}$ (isto para reduzir o costo computacional). Nesse sentido, definimos o espaço de volumes finitos como

$$
\widetilde{V}_{h}(\widetilde{\mathcal{V}}):=\left\{v \in L^{2}\left(\mathbb{S}^{2}\right):\left.v\right|_{\widetilde{\mathrm{V}}_{i}} \text { é constante em cada } \widetilde{\mathrm{V}}_{i} \operatorname{com} i=1, \ldots, N\right\} .
$$

Em outras palavras, $\widetilde{V}_{h}$ está formado pelas funções constantes por pedaços em $\mathbb{S}^{2}$. Cada função $v \in \widetilde{V}_{h}$ pode ser representada por,

$$
v(\mathrm{x})=\sum_{i=1}^{N} v\left(\mathrm{x}_{i}\right) \chi_{i}(\mathrm{x})
$$

onde $\chi_{i}$ denota a função característica associada à célula de Voronoï $\widetilde{V}_{i}$. Note-se que $\widetilde{V}_{h}$ possui a mesma dimensão do espaço $\widetilde{U}_{h}$, i.e., $\operatorname{Dim}\left(\widetilde{V}_{h}\right)=\operatorname{Dim}\left(\widetilde{U}_{h}\right)=N$, mas $\widetilde{V}_{h} \not \subset H_{Q}^{1}\left(\mathbb{S}^{2}\right)$. Essa diferença entre os espaços $\widetilde{U}_{h}$ e $\widetilde{V}_{h}$ é uma dificuldade na obtenção de estimativas de convergência nos MVF, mas é usual nos MEFL (Chou e Ye, 2007; Li et al., 2000).

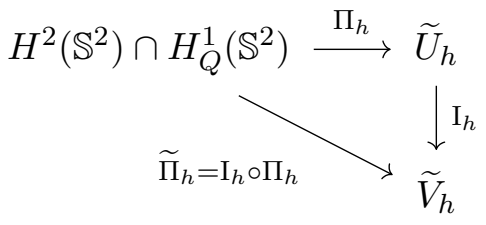

Figura 3.4: Estrutura dos operadores lineares $\Pi_{h}$ associado ao espaço $\widetilde{U}_{h}, \widetilde{\Pi}_{h}$ associado ao espaço $\widetilde{V}_{h}$ e $\mathrm{I}_{h}$ o interpolador linear de transferência entre os espaços $\widetilde{U}_{h}$ e $\widetilde{V}_{h}$.

Definidos os espaços, queremos agora reescrever o esquema de volumes finitos (3.11) como se fosse um MEFL em superfícies (sob alguma regra de integração particular), e usar a teoria de aproximação disponível dos MEFL. A construção é feita com base na relação dos operadores lineares de interpolação associados a cada um dos espaços. Então, sejam $\Pi_{h}: H^{2}\left(\mathbb{S}^{2}\right) \cap H_{Q}^{1}\left(\mathbb{S}^{2}\right) \rightarrow \widetilde{U}_{h}$ o operador linear de interpolação em $\widetilde{U}_{h}$ e $\widetilde{\Pi}_{h}: H^{2}\left(\mathbb{S}^{2}\right) \cap H_{Q}^{1}\left(\mathbb{S}^{2}\right) \rightarrow \widetilde{V}_{h}$ o operador linear de interpolação em $\widetilde{V}_{h}$. Em particular, a seguinte proposição determina um resultado essencial de aproximação em $\widetilde{U}_{h}$. A demonstração está contida no artigo de Demlow (2009, Proposição 2.7, pp. 812).

Proposição 3.10 (Demlow, 2009). Sejam $2 \leq p \leq \infty$ e $u \in W^{2, p}\left(\mathbb{S}^{2}\right) \cap H_{Q}^{1}\left(\mathbb{S}^{2}\right)$. Então, para $h$ suficientemente pequeno, existe uma constante $C \in \mathbb{R}_{+}$independente de $h$ tal que,

$$
\left\|u-\Pi_{h}(u)\right\|_{W^{k, p}\left(\mathbb{S}^{2}\right)} \leq C h^{2-k}\|u\|_{W^{2, p}\left(\mathbb{S}^{2}\right)}, \quad \text { para cada } k=0,1 .
$$

Observação 3.4. Um fato de interesse no interpolador linear $\Pi_{h}$ é a sua invariância para qualquer função $v_{h} \in \widetilde{U}_{h}$, i.e., $\Pi_{h}\left(v_{h}\right)=v_{h}$ e ademais é limitado: para cada $v \in H^{1}\left(\mathbb{S}^{2}\right)$, existe uma constante $C \in \mathbb{R}_{+}$, que depende só da regularidade da malha, tal que $\left\|\Pi_{h}(v)\right\|_{H^{1}\left(\mathbb{S}^{2}\right)} \leq C\|v\|_{H^{1}\left(\mathbb{S}^{2}\right)}$. A prova do anterior está detalhada para domínios do $\mathbb{R}^{n+1}(n=2,3)$ no texto de Brenner e Scott (200\%, Teorema 4.8.12, pp 123).

Observação 3.5. A aproximação dada pelo interpolador $\widetilde{\Pi}_{h}: H^{2}\left(\mathbb{S}^{2}\right) \cap H_{Q}^{1}\left(\mathbb{S}^{2}\right) \rightarrow \widetilde{V}_{h}$, será dada na Proposição 4.1.

Seguindo no contexto, é natural construir um operador que aplique funções de $\widetilde{U}_{h}$ sob $\widetilde{V}_{h}$. Consideremos $\mathrm{I}_{h}: \widetilde{U}_{h} \rightarrow \widetilde{V}_{h}$ o interpolador linear de transferência um-a-um (Figura 3.4), definido como

$$
\mathrm{I}_{h}\left(v_{h}\right)(\mathrm{x})=\sum_{i=1}^{N} v_{h}\left(\mathrm{x}_{i}\right) \chi_{i}(\mathrm{x}), \quad \text { para cada } v_{h} \in \widetilde{U}_{h} .
$$


Como queremos estabelecer estimações com o interpolador $\mathrm{I}_{h}$ será necessário invocar a propriedade inversa dos MEFL válida para decomposições quase-uniformes. A prova está descrita para problemas no $\mathbb{R}^{3}$ nos textos de Ciarlet (2002, Teorema 3.2.6, pp. 140) ou Brenner e Scott (2007, Teorema 4.5.11, pp. 112), mas é válida na esfera sob as condições das Proposições 3.8 e 3.9 (veja Demlow, 2009, Proposição 2.7, pp. 812).

Lema 3.2 (Propriedade inversa, Ciarlet, 2002). Seja $\widetilde{\mathcal{T}}_{h}\left(\mathbb{S}^{2}\right)$ uma família de triangulações definidas em $\mathbb{S}^{2}$ que satisfazem as condições $(2.5 \mathrm{a})$ e $(2.5 \mathrm{~b})$. Assuma que $\widetilde{U}_{h}$ é o espaço de elementos finitos associado à decomposição primal $\widetilde{\mathcal{T}}_{h}$ de $\mathbb{S}^{2}$, que $l, m$ são inteiros não negativos e $1 \leq r, t \leq \infty$, tal que

$$
l \leq m, \quad \widetilde{U}_{h} \subset W^{l, r}\left(\widetilde{\mathrm{T}}_{i j k}\right) \cap W^{m, t}\left(\widetilde{\mathrm{T}}_{i j k}\right) .
$$

Então, existe uma constante $C \in \mathbb{R}_{+}$independente do parâmetro $h$, tal que para cada $v_{h} \in \widetilde{U}_{h}$, tem-se

$$
\left(\sum_{\widetilde{T}_{i j k} \in \widetilde{\mathcal{T}}_{h}}\left\|v_{h}\right\|_{W^{m, t}\left(\widetilde{\mathrm{T}}_{i j k}\right)}^{t}\right)^{1 / t} \leq \frac{C}{\left(h^{2}\right)^{\max \left\{0, \frac{1}{r}-\frac{1}{t}\right\}} h^{m-l}}\left(\sum_{\widetilde{\mathrm{T}}_{i j k} \in \widetilde{\mathcal{T}}_{h}}\left\|v_{h}\right\|_{W^{l, r}\left(\widetilde{\mathrm{T}}_{i j k}\right)}^{r}\right)^{1 / r} .
$$

Em particular, para $r=2, t=\infty$ e $l=m=1$, temos

$$
\left\|\nabla_{s} v_{h}\right\|_{L^{\infty}\left(\widetilde{\mathrm{T}}_{i j k}\right)} \leq C h^{-1}\left\|\nabla_{s} v_{h}\right\|_{L^{2}\left(\widetilde{\mathrm{T}}_{i j k}\right)}
$$

e para $r=1$,

$$
\left\|\nabla_{s} v_{h}\right\|_{L^{\infty}\left(\widetilde{T}_{i j k}\right)} \leq C h^{-2}\left\|\nabla_{s} v_{h}\right\|_{L^{1}\left(\widetilde{T}_{i j k}\right)} .
$$

Algumas características do interpolador $\mathrm{I}_{h}$ foram descritas por Chou et al. (2003); Zhang (2014) para problemas no plano. Examinaremos e mostraremos que são válidas em $\mathbb{S}^{2}$ para decomposições Voronoï-Delaunay, e contribuirão na limitação do Lema 4.1.

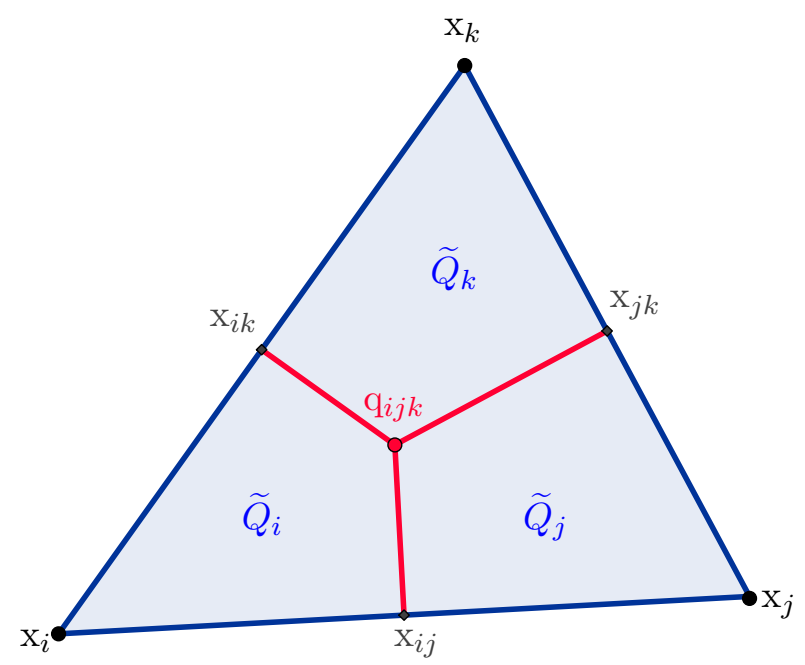

Figura 3.5: Configuração geométrica do triângulo esférico $\widetilde{\mathrm{T}}_{i j k} \in \widetilde{\mathcal{T}}_{h}$ com vértices $\mathrm{x}_{i}, \mathrm{x}_{j}$ e $\mathrm{x}_{k}$. Em vermelho o circuncentro $\mathrm{q}_{i j k}$ e as arestas da malha dual que se intersectam com o interior do triângulo $\widetilde{\mathrm{T}}_{i j k}$.

Lema 3.3. Sejam $\widetilde{\mathrm{T}}_{i j k} \in \widetilde{\mathcal{T}}_{h}$ o triângulo esférico e $\widetilde{\tau}_{i j} \subset \partial \widetilde{\mathrm{T}}_{i j k}$ que satisfazem as condições (2.5b) $e(2.5 \mathrm{a})$ respectivamente. Então, para cada $1 \leq q \leq \infty$ e $v_{h} \in \widetilde{U}_{h}$, existe uma constante $C \in \mathbb{R}_{+}$ 
independente de $h$, tal que

$$
\begin{aligned}
\int_{\widetilde{\tau}_{i j}}\left[v_{h}(\mathrm{x})-\mathrm{I}_{h}\left(v_{h}\right)(\mathrm{x})\right] d \gamma(\mathrm{x}) & =0, \\
\left\|v_{h}-\mathrm{I}_{h}\left(v_{h}\right)\right\|_{L^{q}\left(\widetilde{\mathrm{T}}_{i j k}\right)} & \leq C h\left\|v_{h}\right\|_{W^{1, q}\left(\widetilde{\mathrm{T}}_{i j k}\right)},
\end{aligned}
$$

Demonstração. Para (3.18a) basta mostrar que o termo $v_{h}-\mathrm{I}_{h}\left(v_{h}\right)$ é ímpar em relação ao ponto médio de cada aresta de $\widetilde{\mathrm{T}}_{i j k}$. Com efeito, dado $\mathrm{x}_{i j}$ o ponto médio da aresta $\widetilde{\tau}_{i j} \subset \partial \widetilde{\mathrm{T}}_{i j k}$, definamos $\widetilde{\tau}_{i j}^{(n)}$ a distância geodésica entre os pontos $\mathrm{x}_{i j}$ e $\mathrm{x}_{n} \operatorname{com} n=i, j$. Sabemos que $\mathrm{I}_{h}\left(v_{h}\right)(\mathrm{x})=v_{h}\left(\mathrm{x}_{n}\right)$ para cada $\mathrm{x} \in \widetilde{\tau}_{i j}^{(n)}(n=i, j)$. Aliás, $\widetilde{\tau}_{i j}=\widetilde{\tau}_{i j}^{(i)} \cup \widetilde{\tau}_{i j}^{(j)}$, então temos a expressão,

$$
\begin{aligned}
\int_{\widetilde{\tau}_{i j}}\left[v_{h}(\mathrm{x})-\mathrm{I}_{h}\left(v_{h}\right)(\mathrm{x})\right] d \gamma(\mathrm{x}) & =\int_{\widetilde{\tau}_{i j}^{(i)}}\left[v_{h}(\mathrm{x})-v_{h}\left(\mathrm{x}_{i}\right)\right] d \gamma(\mathrm{x})+\int_{\widetilde{\tau}_{i j}^{(j)}}\left[v_{h}(\mathrm{x})-v_{h}\left(\mathrm{x}_{j}\right)\right] d \gamma(\mathrm{x}) \\
& =\int_{\widetilde{\tau}_{i j}} v_{h}(\mathrm{x}) d \gamma(\mathrm{x})-\int_{\widetilde{\tau}_{i j}^{(i)}} v_{h}\left(\mathrm{x}_{i}\right) d \gamma(\mathrm{x})-\int_{\widetilde{\tau}_{i j}^{(j)}} v_{h}\left(\mathrm{x}_{j}\right) d \gamma(\mathrm{x}) \\
& =\int_{\widetilde{\tau}_{i j}} v_{h}(\mathrm{x}) d \gamma(\mathrm{x})-\frac{1}{2} m\left(\widetilde{\tau}_{i j}\right)\left[v_{h}\left(\mathrm{x}_{i}\right)+v_{h}\left(\mathrm{x}_{j}\right)\right]
\end{aligned}
$$

onde $m\left(\widetilde{\tau}_{i j}\right)$ denota o cumprimento da aresta $\widetilde{\tau}_{i j}$. Decorre da integração numérica que a regra de ponto médio é exata para funções lineares. Com efeito, consideremos $v_{h}^{\Omega}$ a extensão de $v_{h}$ a $\Omega_{i j k}:=\left\{\mathrm{x}^{*} \in \Omega_{h}: \mathcal{P}\left(\mathrm{x}^{*}\right) \in \widetilde{\mathrm{T}}_{i j k}\right\}$, com $v_{h}(\mathrm{x})=v_{h}^{\Omega}(\mathrm{x})$ para cada $\mathrm{x} \in \mathbb{S}^{2}$ e $\mathrm{x}_{i j}^{*}$ o ponto médio de $\left[\mathrm{x}_{i}, \mathrm{x}_{j}\right]$ (o segmento que liga $\mathrm{x}_{i}$ a $\mathrm{x}_{j}$ ) tal que $\mathrm{x}_{i j}=\mathcal{P}\left(\mathrm{x}_{i j}^{*}\right)$. Assumamos que $v_{h}^{\Omega} \in C^{1}\left(\Omega_{i j k}\right)$, então usando o Teorema de Taylor para $v_{h}^{\Omega}$ em torno de $\mathrm{x}_{i j}$ e integrando em relação a $\widetilde{\tau}_{i j}$, resulta

$\int_{\widetilde{\tau}_{i j}} v_{h}(\mathrm{x}) d \gamma(\mathrm{x})=\int_{\widetilde{\tau}_{i j}} v_{h}^{\Omega}(\mathrm{x}) d \gamma(\mathrm{x})=m\left(\widetilde{\Gamma}_{i j}\right) v_{h}^{\Omega}\left(\mathrm{x}_{i j}\right)+\int_{\widetilde{\tau}_{i j}} \int_{0}^{1} \nabla v_{h}^{\Omega}\left(\mathrm{x}_{i j}+t\left(\mathrm{x}-\mathrm{x}_{i j}\right)\right) \cdot\left(\mathrm{x}-\mathrm{x}_{i j}\right) d t d \gamma(\mathrm{x})$.

O segundo termo no lado direito anula-se por conta da paridade de $\nabla v_{h}^{\Omega}(\mathrm{y})$ para qualquer $\mathrm{y} \in\left[\mathrm{x}, \mathrm{x}_{i j}\right]$ e a simetria na aresta em relação ao ponto médio $\mathrm{x}_{i j}$. Logo,

$$
\int_{\widetilde{\tau}_{i j}} v_{h}(\mathrm{x}) d \gamma(\mathrm{x})=m\left(\widetilde{\Gamma}_{i j}\right) v_{h}^{\Omega}\left(\mathrm{x}_{i j}\right)
$$

Substituindo a expressão acima em (3.19) obtemos

$$
\int_{\widetilde{\tau}_{i j}}\left[v_{h}(\mathrm{x})-\mathrm{I}_{h}\left(v_{h}\right)(\mathrm{x})\right] d \gamma(\mathrm{x})=m\left(\widetilde{\tau}_{i j}\right) v_{h}^{\Omega}\left(\mathrm{x}_{i j}\right)-\frac{1}{2} m\left(\widetilde{\tau}_{i j}\right)\left[v_{h}\left(\mathrm{x}_{i}\right)+v_{h}\left(\mathrm{x}_{j}\right)\right]=0,
$$

dado que $v_{h}^{\Omega}\left(\mathrm{x}_{i j}\right)=v_{h}^{\Omega}\left(\mathrm{x}_{i j}^{*}\right)$ e $v_{h}^{\Omega}\left(\mathrm{x}_{i j}^{*}\right)=\frac{1}{2}\left[v_{h}\left(\mathrm{x}_{i}\right)+v_{h}\left(\mathrm{x}_{j}\right)\right]$. O que prova a identidade (3.18a).

Para (3.18b), conforme ilustrado na Figura 3.5, consideremos $\widetilde{\mathrm{Q}}_{i}, \widetilde{\mathrm{Q}}_{j}$ e $\widetilde{\mathrm{Q}}_{k}$ as regiões poligonais esféricas formadas pela intersecção do triângulo $\widetilde{T}_{i j k}$ com as células de Voronoï associadas a cada um dos vértices $\mathrm{x}_{i}, \mathrm{x}_{j}$ e $\mathrm{x}_{k}$, i.e.,

$$
\widetilde{\mathrm{Q}}_{n}=\widetilde{\mathrm{T}}_{i j k} \cap \widetilde{\mathrm{V}}_{n}, \quad \operatorname{com} n=i, j, k .
$$

Em particular, se tomamos $\widetilde{\mathrm{Q}}_{i}$, temos $\mathrm{I}_{h}\left(v_{h}\right)(\mathrm{x})=v_{h}\left(\mathrm{x}_{i}\right)$ para cada $\mathrm{x} \in \widetilde{\mathrm{Q}}_{i}$, calculamos

$$
\left|v_{h}(\mathrm{x})-v_{h}\left(\mathrm{x}_{i}\right)\right|=\left|\int_{0}^{1} \nabla v_{h}^{\Omega}\left(\mathrm{x}_{i}+t\left(\mathrm{x}-\mathrm{x}_{i}\right)\right) \cdot\left(\mathrm{x}-\mathrm{x}_{i}\right) d t\right| \leq\left|\mathrm{x}-\mathrm{x}_{i}\right| \max _{\mathrm{x}^{*} \in\left[\mathrm{x}, \mathrm{x}_{i}\right]}\left|\nabla v_{h}^{\Omega}\left(\mathrm{x}^{*}\right)\right|,
$$


onde $\left[\mathrm{x}, \mathrm{x}_{i}\right]$ é o segmento que liga $\mathrm{x}$ a $\mathrm{x}_{i}$. Para $1 \leq q \leq \infty$ integramos em relação a $\widetilde{\mathrm{Q}}_{i}$, o que resulta

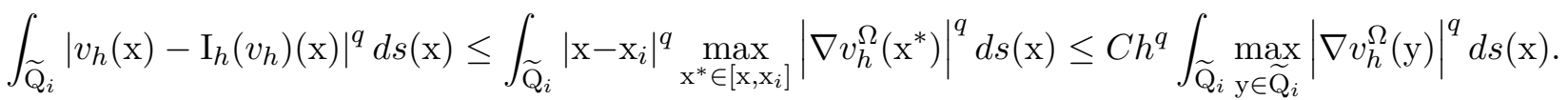

Então, pondo $l=m=1, r=q$ e $t=\infty$ na propriedade inversa (Lema 3.2), junto à condição (2.5b), obtemos

$$
\left\|v_{h}-\mathrm{I}_{h}\left(v_{h}\right)\right\|_{L^{q}\left(\widetilde{\mathrm{Q}}_{i}\right)}^{q} \leq C h^{q} S\left(\widetilde{\mathrm{Q}}_{i}\right)\left|\nabla v_{h}^{\Omega}\right|_{L^{\infty}\left(\widetilde{\mathrm{Q}}_{i}\right)}^{q} \leq C h^{q}\left\|\nabla v_{h}^{\Omega}\right\|_{L^{q}\left(\widetilde{\mathrm{Q}}_{i}\right)}^{q} .
$$

De maneira semelhante, esse processo pode ser aplicado nas regiões restantes. Em consequência, obtemos

$$
\left\|v_{h}-\mathrm{I}_{h}\left(v_{h}\right)\right\|_{L^{q}\left(\widetilde{\mathrm{T}}_{i j k}\right)}=\left(\sum_{n=i, j, k}\left\|v_{h}-\mathrm{I}_{h}\left(v_{h}\right)\right\|_{L^{q}\left(\widetilde{\mathrm{Q}}_{n}\right)}^{q}\right)^{1 / q} \leq C h\left\|v_{h}\right\|_{W^{1, q}\left(\widetilde{\mathrm{T}}_{i j k}\right)},
$$

como queríamos mostrar. Isto encerra a demonstração.

Deve-se notar que a exigência na malha ser quase-uniforme é essencial para a validez do lema anterior. Podemos agora associar algumas normas discretas usuais à decomposição dual $\widetilde{\mathcal{V}}=\left\{\mathrm{x}_{i}, \widetilde{\mathrm{V}}_{i}\right\}_{i=1}^{N}$ para funções de $\widetilde{U}_{h}$, definidas por Du et al. (2003b) e Mishev (1998).

Definição 3.4 (Normas discretas). Para $u_{h}, v_{h} \in \widetilde{U}_{h}$ e $\mathrm{I}_{h}: \widetilde{U}_{h} \rightarrow \widetilde{V}_{h}$ o interpolador linear de transferência, tem-se que

$$
\begin{aligned}
\left\|u_{h}\right\|_{0, h}^{2} & =\left\langle\mathrm{I}_{h}\left(u_{h}\right), \mathrm{I}_{h}\left(u_{h}\right)\right\rangle_{h}, \quad\left\langle\mathrm{I}_{h}\left(u_{h}\right), \mathrm{I}_{h}\left(v_{h}\right)\right\rangle_{h}=\sum_{i=1}^{N} S\left(\widetilde{\mathrm{V}}_{i}\right) u_{h}\left(\mathrm{x}_{i}\right) v_{h}\left(\mathrm{x}_{i}\right) \\
\left|u_{h}\right|_{1, h}^{2} & =\frac{1}{2} \sum_{i=1}^{M} \sum_{j \in \Lambda_{i}} m\left(\widetilde{\Gamma}_{i j}\right) \mathrm{d}\left(\mathrm{x}_{i}, \mathrm{x}_{j}\right)\left|\frac{u_{h}\left(\mathrm{x}_{i}\right)-u_{h}\left(\mathrm{x}_{j}\right)}{\left\|\mathrm{x}_{i}-\mathrm{x}_{j}\right\|}\right|^{2} \\
\left\|u_{h}\right\|_{1, h}^{2} & =\left\|u_{h}\right\|_{0, h}^{2}+\left|u_{h}\right|_{1, h}^{2} \\
\left\|u_{h}\right\|_{\infty, h} & =\max _{\mathrm{x}_{i} \in \mathrm{S}_{N}}\left|u_{h}\left(\mathrm{x}_{i}\right)\right|
\end{aligned}
$$

onde $\mathrm{S}_{N}=\left\{\mathrm{x}_{i}\right\}_{i=1}^{N}$ é o conjunto de vértices em $\mathbb{S}^{2}$.

Além disso, devido as Proposições 3.7 e 3.9, temos agora uma equivalência entre as normas:

Proposição 3.11 (Du e Ju, 2005). Para qualquer $u_{h} \in \widetilde{U}_{h}$, existem constantes $C_{n} \in \mathbb{R}_{+}$, com $n \in\{0,1\}$, que independem de $h$, tal que

$$
\begin{aligned}
& \frac{1}{C_{0}}\left\|u_{h}\right\|_{0, h} \leq\left\|u_{h}\right\|_{L^{2}\left(\mathbb{S}^{2}\right)} \leq C_{0}\left\|u_{h}\right\|_{0, h}, \\
& \frac{1}{C_{1}}\left\|u_{h}\right\|_{1, h} \leq\left\|u_{h}\right\|_{H^{1}\left(\mathbb{S}^{2}\right)} \leq C_{1}\left\|u_{h}\right\|_{1, h} .
\end{aligned}
$$

Demonstração. Consideremos que a norma $\|\cdot\|_{0, h}$ pode ser definida como

$$
\left\|u_{h}\right\|_{0, h}:=\left\|\mathrm{I}_{h}\left(u_{h}\right)\right\|_{L^{2}\left(\mathbb{S}^{2}\right)}=\left(\sum_{i=1}^{N} S\left(\widetilde{\mathrm{V}}_{i}\right)\left|u_{h}\left(\mathrm{x}_{i}\right)\right|^{2}\right)^{1 / 2}, \quad \text { para cada } u_{h} \in \widetilde{U}_{h} .
$$

Do fato que o operador $\mathrm{I}_{h}$ é um operador limitado $\left(\widetilde{U}_{h}\right.$ e $\widetilde{V}_{h}$ possuem dimensão finita), então existe uma constante positiva $C_{0} \in \mathbb{R}_{+}$tal que

$$
\left\|u_{h}\right\|_{0, h} \leq C_{0}\left\|u_{h}\right\|_{L^{2}\left(\mathbb{S}^{2}\right)}
$$


Em consequência, temos a desigualdade do lado esquerdo de (3.20a). Analogamente, podemos deduzir o lado direito e as desigualdades em (3.20b).

\subsubsection{Estabilidade}

De posse da informação anterior, podemos definir uma forma variacional para o esquema (3.11). Para $\left(u_{h}, v_{h}\right) \in \widetilde{U}_{h} \times \widetilde{U}_{h}$, definimos os funcionais bilineares $\mathcal{A}_{h}: \widetilde{U}_{h} \times \widetilde{V}_{h} \rightarrow \mathbb{R}$, tal que

$$
\mathcal{A}_{h}\left(u_{h}, \mathrm{I}_{h}\left(v_{h}\right)\right)=\sum_{i=1}^{N} v_{h}\left(\mathrm{x}_{i}\right) \sum_{j \in \Lambda(i)} \mathrm{F}_{i j}^{c}\left(u_{h}\right)
$$

e a versão discreta $\mathcal{A}_{h}^{d}: \widetilde{U}_{h} \times \widetilde{V}_{h} \rightarrow \mathbb{R}$

$$
\mathcal{A}_{h}^{d}\left(u_{h}, \mathrm{I}_{h}\left(v_{h}\right)\right)=\sum_{i=1}^{N} v_{h}\left(\mathrm{x}_{i}\right) \sum_{j \in \Lambda(i)} \mathrm{F}_{i j}^{d}\left(u_{h}\right)
$$

onde $\mathrm{F}_{i j}^{c}$ e $\mathrm{F}_{i j}^{d}$ são os fluxos contínuo e discreto definidos em (3.3) e (3.8) respectivamente. Então, uma aproximação $u_{h}$ de (3.11) é definida como a única solução ao problema discreto: encontrar $u_{h} \in \widetilde{U}_{h}$ tal que

$$
\mathcal{A}_{h}^{d}\left(u_{h}, \mathrm{I}_{h}\left(v_{h}\right)\right)=\left\langle f_{h}, \mathrm{I}_{h}\left(v_{h}\right)\right\rangle_{h}, \quad \text { para cada } v_{h} \in \widetilde{U}_{h} .
$$

Observe que, o produto $\left\langle f_{h}, \mathrm{I}_{h}\left(v_{h}\right)\right\rangle_{h}$ está bem definido, visto que a aproximação $f_{h}$ pertence ao espaço $\widetilde{V}_{h}$ e cujas componentes são dadas pelos valores $f_{i}(i=1, \ldots, N)$ em (3.9). A fim de provar a estabilidade, mostramos um resultado clássico referente à boa colocação discreta do problema (3.23). Mais precisamente:

Proposição 3.12 (Continuidade e Coercividade). Sejam a decomposição Voronö̈-Delaunay que satisfaz as condições (2.5a)-(2.5c) e o fluxo discreto $\mathrm{F}_{i j}^{c}$ definido em (3.8). Então, para cada $u_{h} \in \widetilde{U}_{h}$ que satisfaz o problema (3.23), existem constantes $C_{n} \in \mathbb{R}_{+}$, com $n \in\{0,1\}$, tal que

$$
\begin{aligned}
& \mathcal{A}_{h}^{d}\left(u_{h}, \mathrm{I}_{h}\left(v_{h}\right)\right) \leq C_{0}\left\|u_{h}\right\|_{1, h}\left\|v_{h}\right\|_{1, h}, \quad \text { para cada } v_{h} \in \widetilde{U}_{h}, \\
& \mathcal{A}_{h}^{d}\left(u_{h}, \mathrm{I}_{h}\left(u_{h}\right)\right) \geq C_{1}\left\|u_{h}\right\|_{1, h}^{2} .
\end{aligned}
$$

Demonstração. Mostraremos a continuidade (3.24a). Com efeito, dado o funcional bilinear $\mathcal{A}_{h}^{d}$ : $\widetilde{U}_{h} \times \widetilde{V}_{h} \rightarrow \mathbb{R}$, temos para $u_{h}, v_{h} \in \widetilde{U}_{h}$ que

$$
\mathcal{A}_{h}^{d}\left(u_{h}, \mathrm{I}_{h}\left(v_{h}\right)\right)=\sum_{i=1}^{N} \sum_{j \in \Lambda(i)} \mathrm{F}_{i j}^{d}(u) v_{h}\left(\mathrm{x}_{i}\right)=-\sum_{i=1}^{N} \sum_{j \in \Lambda(i)} m\left(\widetilde{\Gamma}_{i j}\right) \frac{u_{h}\left(\mathrm{x}_{j}\right)-u_{h}\left(\mathrm{x}_{i}\right)}{\left\|\mathrm{x}_{j}-\mathrm{x}_{i}\right\|} v_{h}\left(\mathrm{x}_{i}\right) .
$$

Pela propriedade de conservação discreta, Definição 3.2, tem-se

$$
\begin{aligned}
\mathcal{A}_{h}^{d}\left(u_{h}, \mathrm{I}_{h}\left(v_{h}\right)\right) & =-\sum_{i=1}^{N} \sum_{j \in \Lambda(i)} m\left(\widetilde{\Gamma}_{i j}\right) \frac{u_{h}\left(\mathrm{x}_{j}\right)-u_{h}\left(\mathrm{x}_{i}\right)}{\left\|\mathrm{x}_{j}-\mathrm{x}_{i}\right\|} v_{h}\left(\mathrm{x}_{i}\right) \\
& =\frac{1}{2} \sum_{i=1}^{N} \sum_{j \in \Lambda(i)} m\left(\widetilde{\Gamma}_{i j}\right) \frac{u_{h}\left(\mathrm{x}_{j}\right)-u_{h}\left(\mathrm{x}_{i}\right)}{\left\|\mathrm{x}_{j}-\mathrm{x}_{i}\right\|}\left[v_{h}\left(\mathrm{x}_{j}\right)-v_{h}\left(\mathrm{x}_{i}\right)\right] \\
& =\frac{1}{2} \sum_{i=1}^{N} \sum_{j \in \Lambda(i)} m\left(\widetilde{\Gamma}_{i j}\right)\left\|\mathrm{x}_{j}-\mathrm{x}_{i}\right\| \frac{u_{h}\left(\mathrm{x}_{j}\right)-u_{h}\left(\mathrm{x}_{i}\right)}{\left\|\mathrm{x}_{j}-\mathrm{x}_{i}\right\|} \frac{v_{h}\left(\mathrm{x}_{j}\right)-v_{h}\left(\mathrm{x}_{i}\right)}{\left\|\mathrm{x}_{j}-\mathrm{x}_{i}\right\|}
\end{aligned}
$$


sabendo-se que $0<\left\|\mathrm{x}_{j}-\mathrm{x}_{i}\right\| \leq \mathrm{d}\left(\mathrm{x}_{i}, \mathrm{x}_{j}\right)$, segue

$$
\begin{aligned}
\mathcal{A}_{h}^{d}\left(u_{h}, \mathrm{I}_{h}\left(v_{h}\right)\right) & \leq \frac{1}{2} \sum_{i=1}^{N} \sum_{j \in \Lambda(i)} m\left(\widetilde{\Gamma}_{i j}\right) \mathrm{d}\left(\mathrm{x}_{i}, \mathrm{x}_{j}\right) \frac{u_{h}\left(\mathrm{x}_{j}\right)-u_{h}\left(\mathrm{x}_{i}\right)}{\left\|\mathrm{x}_{j}-\mathrm{x}_{i}\right\|} \frac{v_{h}\left(\mathrm{x}_{j}\right)-v_{h}\left(\mathrm{x}_{i}\right)}{\left\|\mathrm{x}_{j}-\mathrm{x}_{i}\right\|} \\
& =\sum_{i=1}^{N} \sum_{j \in \Lambda(i)}\left(\sqrt{\frac{1}{2} m\left(\widetilde{\Gamma}_{i j}\right) \mathrm{d}\left(\mathrm{x}_{i}, \mathrm{x}_{j}\right)}\right)^{2} \frac{u_{h}\left(\mathrm{x}_{j}\right)-u_{h}\left(\mathrm{x}_{i}\right)}{\left\|\mathrm{x}_{j}-\mathrm{x}_{i}\right\|} \frac{v_{h}\left(\mathrm{x}_{j}\right)-v_{h}\left(\mathrm{x}_{i}\right)}{\left\|\mathrm{x}_{j}-\mathrm{x}_{i}\right\|} .
\end{aligned}
$$

Resulta imediatamente da desigualdade de Cauchy-Schwarz,

$$
\begin{aligned}
\mathcal{A}_{h}^{d}\left(u_{h}, \mathrm{I}_{h}\left(v_{h}\right)\right) \leq & C\left(\sum_{i=1}^{N} \sum_{j \in \Lambda(i)} \frac{1}{2} m\left(\widetilde{\Gamma}_{i j}\right) \mathrm{d}\left(\mathrm{x}_{i}, \mathrm{x}_{j}\right)\left|\frac{u_{h}\left(\mathrm{x}_{j}\right)-u_{h}\left(\mathrm{x}_{i}\right)}{\left\|\mathrm{x}_{j}-\mathrm{x}_{i}\right\|}\right|^{2}\right)^{1 / 2} \\
& \times\left(\sum_{i=1}^{N} \sum_{j \in \Lambda(i)} \frac{1}{2} m\left(\widetilde{\Gamma}_{i j}\right) \mathrm{d}\left(\mathrm{x}_{i}, \mathrm{x}_{j}\right)\left|\frac{v_{h}\left(\mathrm{x}_{j}\right)-v_{h}\left(\mathrm{x}_{i}\right)}{\left\|\mathrm{x}_{j}-\mathrm{x}_{i}\right\|}\right|^{2}\right)^{1 / 2} \\
\leq & C\left|u_{h}\right|_{1, h}\left|v_{h}\right|_{1, h} .
\end{aligned}
$$

Da construção de $\widetilde{U}_{h}$, a seminorma é uma norma, o que provaria (3.24a). Agora, para a coercividade (3.24b), temos

$$
\mathcal{A}_{h}^{d}\left(u_{h}, \mathrm{I}_{h}\left(u_{h}\right)\right)=-\sum_{i=1}^{N} \sum_{j \in \Lambda(i)} m\left(\widetilde{\Gamma}_{i j}\right) \frac{u_{h}\left(\mathrm{x}_{j}\right)-u_{h}\left(\mathrm{x}_{i}\right)}{\left\|\mathrm{x}_{j}-\mathrm{x}_{i}\right\|} u_{h}\left(\mathrm{x}_{i}\right) .
$$

Usando de novo a conservação discreta e a relação $\left\|\mathrm{x}_{j}-\mathrm{x}_{i}\right\| \geq \frac{1}{2} \mathrm{~d}\left(\mathrm{x}_{j}, \mathrm{x}_{i}\right)$ produzem

$$
\begin{aligned}
\mathcal{A}_{h}^{d}\left(u_{h}, \mathrm{I}_{h}\left(u_{h}\right)\right) & =\frac{1}{2} \sum_{i=1}^{N} \sum_{j \in \Lambda(i)} m\left(\widetilde{\Gamma}_{i j}\right) \frac{u_{h}\left(\mathrm{x}_{j}\right)-u_{h}\left(\mathrm{x}_{i}\right)}{\left\|\mathrm{x}_{j}-\mathrm{x}_{i}\right\|}\left[u_{h}\left(\mathrm{x}_{j}\right)-u_{h}\left(\mathrm{x}_{i}\right)\right] \\
& =\frac{1}{2} \sum_{i=1}^{N} \sum_{j \in \Lambda(i)} m\left(\widetilde{\Gamma}_{i j}\right)\left\|\mathrm{x}_{j}-\mathrm{x}_{i}\right\|\left|\frac{u_{h}\left(\mathrm{x}_{j}\right)-u_{h}\left(\mathrm{x}_{i}\right)}{\left\|\mathrm{x}_{j}-\mathrm{x}_{i}\right\|}\right|^{2} \\
& \geq \frac{1}{2} \sum_{i=1}^{N} \sum_{j \in \Lambda(i)} \frac{1}{2} m\left(\widetilde{\Gamma}_{i j}\right) \mathrm{d}\left(\mathrm{x}_{i}, \mathrm{x}_{j}\right)\left|\frac{u_{h}\left(\mathrm{x}_{j}\right)-u_{h}\left(\mathrm{x}_{i}\right)}{\left\|\mathrm{x}_{j}-\mathrm{x}_{i}\right\|}\right|^{2} \\
& \geq C\left\|u_{h}\right\|_{1, h}^{2} .
\end{aligned}
$$

Portanto temos (3.24b). Isto encerra a demonstração da proposição.

$\mathrm{Na}$ proposição abaixo enunciamos a estimação referente à estabilidade, o qual ocorre como consequência imediata da Proposição 3.12.

Proposição 3.13 (Estabilidade). Seja $f \in L^{2}\left(\mathbb{S}^{2}\right)$ que satisfaz a condição de compatibilidade (1.3). A única solução $u_{h} \in \widetilde{U}_{h}$ do problema discreto (3.23) satisfaz a condição

$$
\left\|u_{h}\right\|_{1, h} \leq C\|f\|_{L^{2}\left(\mathbb{S}^{2}\right)}
$$

onde $C \in \mathbb{R}_{+}$é uma constante independente de $h$.

Demonstração. Com efeito, das equações (3.24a) e (3.24b) junto com a desigualdade de CauchySchwarz obtemos

$$
\left\|u_{h}\right\|_{1, h}^{2} \leq C\left|\mathcal{A}_{h}^{d}\left(u_{h}, \mathrm{I}_{h}\left(u_{h}\right)\right)\right| \leq C\left|\left\langle f_{h}, \mathrm{I}_{h}\left(u_{h}\right)\right\rangle_{h}\right| \leq C\|f\|_{L^{2}\left(\mathbb{S}^{2}\right)}\left\|u_{h}\right\|_{L^{2}\left(\mathbb{S}^{2}\right)},
$$


pela Proposição 3.11 segue

$$
\left\|u_{h}\right\|_{1, h}^{2} \leq C\|f\|_{L^{2}\left(\mathbb{S}^{2}\right)}\left\|u_{h}\right\|_{H^{1}\left(\mathbb{S}^{2}\right)} \leq C\|f\|_{L^{2}\left(\mathbb{S}^{2}\right)}\left\|u_{h}\right\|_{1, h} .
$$

Dividindo por $\left\|u_{h}\right\|_{1, h}$ cada lado da desigualdade obtemos a limitação desejada.

\subsubsection{Estimações do erro geométrico}

Esta subseção tem como finalidade enunciar duas estimações do erro geométrico produzidas na aproximação de $\mathbb{S}^{2}$ pela sequência de poliedros $\mathbf{S}_{h}$, quando $h$ vai para zero. O seguinte lema foi relatado por Du e Ju (2005, Lema 4, pp. 1686) e mostra uma limitação de ordem dois em relação ao tamanho de malha $h$ para a diferença entre os funcionais bilineares $\mathcal{A}_{h}(\cdot, \cdot)$ e $\mathcal{A}_{h}^{d}(\cdot, \cdot)$. Cabe aclarar que a prova aqui é adaptada para o nosso problema de estudo.

Lema 3.4 (Du e Ju, 2005). Sejam $\mathcal{A}_{h}(\cdot, \cdot)$ e $\mathcal{A}_{h}^{d}(\cdot, \cdot)$ os funcionais bilineares definidos em (3.21) e (3.22) respectivamente. Assuma que $u_{h} \in \widetilde{U}_{h}$ é a única solução do problema discreto (3.23). Então, existe uma constante $C \in \mathbb{R}_{+}$, que independe de $h$, tal que

$$
\left|\mathcal{A}_{h}\left(u_{h}, \mathrm{I}_{h}\left(v_{h}\right)\right)-\mathcal{A}_{h}^{d}\left(u_{h}, \mathrm{I}_{h}\left(v_{h}\right)\right)\right| \leq C h^{2}\left\|u_{h}\right\|_{H^{1}\left(\mathbb{S}^{2}\right)}\left\|v_{h}\right\|_{H^{1}\left(\mathbb{S}^{2}\right)}, \quad \text { para cada } v_{h} \in \widetilde{U}_{h} .
$$

Demonstração. Sejam $u_{h} \in \widetilde{U}_{h}$ e os fluxos contínuo e discreto definidos em (3.3) e (3.8) respectivamente. Então, denotemos por $\xi_{i j}$ o erro entre os fluxos, i.e.,

$$
\begin{aligned}
\xi_{i j}\left(u_{h}\right) & =-\frac{1}{m\left(\widetilde{\Gamma}_{i j}\right)} \int_{\widetilde{\Gamma}_{i j}} \nabla_{s} u_{h}(\mathrm{x}) \cdot \overrightarrow{\mathrm{n}}_{\mathrm{x}, \widetilde{\Gamma}_{i j}} d \gamma(\mathrm{x})+\frac{u_{h}\left(\mathrm{x}_{j}\right)-u_{h}\left(\mathrm{x}_{i}\right)}{\left\|\mathrm{x}_{j}-\mathrm{x}_{i}\right\|} \\
& =-\frac{1}{m\left(\widetilde{\Gamma}_{i j}\right)} \int_{\widetilde{\Gamma}_{i j}} \nabla_{s} u_{h}(\mathrm{x}) \cdot \overrightarrow{\mathrm{n}}_{\mathrm{x}, \widetilde{\Gamma}_{i j}} d \gamma(\mathrm{x})+\nabla u_{h}^{\Omega}\left(\mathrm{x}^{*}\right) \cdot \overrightarrow{\mathrm{n}}_{\mathrm{x}, \widetilde{\Gamma}_{i j}},
\end{aligned}
$$

onde $u_{h}^{\Omega}$ é a extensão de $u_{h}$ a $\Omega_{h}$. Se para cada $\mathrm{x} \in \widetilde{\Gamma}_{i j}$ temos $\mathrm{x}^{*}=\mathcal{P}^{-1}(\mathrm{x})$, então da Proposição 3.8 segue, $\nabla_{s} u_{h}(\mathrm{x})=\nabla u_{h}^{\Omega}(\mathrm{x})=\left\|\mathrm{x}^{*}\right\| \nabla u_{h}^{\Omega}\left(\mathrm{x}^{*}\right)$ e junto ao fato $\left|\left\|\mathrm{x}^{*}\right\|-1\right| \leq C h^{2}$, resulta

$$
\left|\nabla_{s} u_{h}(\mathrm{x})-\nabla u_{h}^{\Omega}\left(\mathrm{x}^{*}\right)\right|=||\left|\mathrm{x}^{*}\right|\left|\nabla u_{h}^{\Omega}(\mathrm{x})-\nabla u_{h}^{\Omega}\left(\mathrm{x}^{*}\right)\right| \leq C h^{2}\left|\nabla u_{h}^{\Omega}\left(\mathrm{x}^{*}\right)\right| .
$$

Assim,

$$
\left|\xi_{i j}\left(u_{h}\right)\right| \leq \frac{1}{m\left(\widetilde{\Gamma}_{i j}\right)} \int_{\widetilde{\Gamma}_{i j}}\left|\left[\nabla_{s} u_{h}(\mathrm{x})-\nabla u_{h}^{\Omega}\left(\mathrm{x}^{*}\right)\right] \cdot \overrightarrow{\mathrm{n}}_{\mathrm{x}, \widetilde{\Gamma}_{i j} \mid}\right| d \gamma(\mathrm{x}) \leq C h^{2} \frac{\left|u_{h}\left(\mathrm{x}_{j}\right)-u_{h}\left(\mathrm{x}_{i}\right)\right|}{\left\|\mathrm{x}_{j}-\mathrm{x}_{i}\right\|}
$$

Desta maneira, a diferença dos funcionais bilineares irá ser

$$
\begin{aligned}
\left|\mathcal{A}_{h}\left(u_{h}, \mathrm{I}_{h}\left(v_{h}\right)\right)-\mathcal{A}_{h}^{d}\left(u_{h}, \mathrm{I}_{h}\left(v_{h}\right)\right)\right| & =\left|\sum_{i=1}^{N} \sum_{j \in \Lambda(i)} v_{h}\left(\mathrm{x}_{i}\right)\left[\mathrm{F}_{i j}^{c}\left(u_{h}\right)-\mathrm{F}_{i j}^{d}\left(u_{h}\right)\right]\right| \\
& =\left|\sum_{i=1}^{N} \sum_{j \in \Lambda(i)} v_{h}\left(\mathrm{x}_{i}\right) m\left(\widetilde{\Gamma}_{i j}\right) \xi_{i j}\left(u_{h}\right)\right| .
\end{aligned}
$$

Agora, para última linha acima, sabemos que o esquema é conservativo e que $\left\|\mathrm{x}_{j}-\mathrm{x}_{i}\right\| \leq \mathrm{d}\left(\mathrm{x}_{i}, \mathrm{x}_{j}\right)$. 
Então, usando a desigualdade de Cauchy-Schwarz e a equação (3.26), obtemos

$$
\begin{aligned}
\sum_{i=1}^{N} \sum_{j \in \Lambda(i)} v_{h}\left(\mathrm{x}_{i}\right) m\left(\widetilde{\Gamma}_{i j}\right) \xi_{i j}\left(u_{h}\right)= & \sum_{i=1}^{N} \sum_{j \in \Lambda(i)} \frac{1}{2} m\left(\widetilde{\Gamma}_{i j}\right)\left\|\mathrm{x}_{j}-\mathrm{x}_{i}\right\| \xi_{i j}\left(u_{h}\right) \frac{v_{h}\left(\mathrm{x}_{j}\right)-v_{h}\left(\mathrm{x}_{i}\right)}{\left\|\mathrm{x}_{j}-\mathrm{x}_{i}\right\|} \\
\leq & \sum_{i=1}^{N} \sum_{j \in \Lambda(i)} \frac{1}{2} m\left(\widetilde{\Gamma}_{i j}\right) \mathrm{d}\left(\mathrm{x}_{i}, \mathrm{x}_{j}\right) \xi_{i j}\left(u_{h}\right) \frac{v_{h}\left(\mathrm{x}_{j}\right)-v_{h}\left(\mathrm{x}_{i}\right)}{\left\|\mathrm{x}_{j}-\mathrm{x}_{i}\right\|} \\
\leq & \left(\sum_{i=1}^{N} \sum_{j \in \Lambda(i)} \frac{1}{2} m\left(\widetilde{\Gamma}_{i j}\right) \mathrm{d}\left(\mathrm{x}_{i}, \mathrm{x}_{j}\right)\left|\xi_{i j}\left(u_{h}\right)\right|^{2}\right)^{1 / 2} \\
& \times\left(\sum_{i=1}^{N} \sum_{j \in \Lambda(i)} \frac{1}{2} m\left(\widetilde{\Gamma}_{i j}\right) \mathrm{d}\left(\mathrm{x}_{i}, \mathrm{x}_{j}\right)\left|\frac{v_{h}\left(\mathrm{x}_{j}\right)-v_{h}\left(\mathrm{x}_{i}\right)}{\left\|\mathrm{x}_{j}-\mathrm{x}_{i}\right\|}\right|^{2}\right)^{1 / 2} \\
\leq & C h^{2}\left\|u_{h}\right\|_{1, h}\left\|v_{h}\right\|_{1, h} .
\end{aligned}
$$

Por fim, usando a equivalência das normas (Proposição 3.11) e combinando (3.27) com (3.28) segue o resultado desejado.

Observação 3.6. Nesta ordem de ideias, é interessante observar que o lema acima é válido para cada $u_{h}, v_{h} \in \widetilde{U}_{h}$, com $p \geq 1,1 / p+1 / q=1$, tem-se pela desigualdade de Hölder a estimativa

$$
\left|\mathcal{A}_{h}\left(u_{h}, \mathrm{I}_{h}\left(v_{h}\right)\right)-\mathcal{A}_{h}^{d}\left(u_{h}, \mathrm{I}_{h}\left(v_{h}\right)\right)\right| \leq C h^{2}\left\|\nabla_{s} u_{h}\right\|_{L^{p}\left(\mathbb{S}^{2}\right)}\left\|\nabla_{s} v_{h}\right\|_{L^{q}\left(\mathbb{S}^{2}\right)}, \quad \text { para cada } v_{h} \in \widetilde{U}_{h} .
$$

O seguinte lema encerrará este capítulo, na prova usaremos os Lemas 3.3 e 3.2. Evidentemente, sua validez é concedida, dado que a decomposição Voronoï-Delaunay é quase-uniforme. O resultado garante uma estimativa de ordem dois na diferença entre os funcionais $\mathcal{A}(\cdot, \cdot)$ e $\mathcal{A}_{h}(\cdot, \cdot)$ restritos para funções no espaço $\widetilde{U}_{h}$.

Lema 3.5. Considere que decomposição Voronö̈-Delaunay em $\mathbb{S}^{2}$ satisfaz as condições (2.5a)(2.5c). Sejam $\mathcal{A}(\cdot, \cdot)$ e $\mathcal{A}_{h}(\cdot, \cdot)$ os funcionais bilineares definidos em (1.5) e (3.21) respectivamente. Seja $u_{h} \in \widetilde{U}_{h}$ a única solução do problema (3.23), Então, existe uma constante $C \in \mathbb{R}_{+}$, que independe de $h$, tal que

$$
\left|\mathcal{A}\left(u_{h}, v_{h}\right)-\mathcal{A}_{h}\left(u_{h}, \mathrm{I}_{h}\left(v_{h}\right)\right)\right| \leq C h^{2}\left\|u_{h}\right\|_{H^{1}\left(\mathbb{S}^{2}\right)}\left\|v_{h}\right\|_{H^{1}\left(\mathbb{S}^{2}\right)}, \quad \text { para cada } v_{h} \in \widetilde{U}_{h} .
$$

Demonstração. Com efeito, dada $u_{h} \in \widetilde{U}_{h}$ assumimos que $\left.u_{h}\right|_{\widetilde{\mathrm{T}}_{i j k}} \in H^{2}\left(\widetilde{\mathrm{T}}_{i j k}\right)$ para cada $\widetilde{\mathrm{T}}_{i j k} \in \widetilde{\mathcal{T}}_{h}$. Então, multiplicando $-\Delta_{s} u_{h}$ por $v_{h} \in \widetilde{U}_{h}$, integrando em relação a $\widetilde{\mathrm{T}}_{i j k}$ e usando a fórmula de Green temos a expressão

$$
-\int_{\widetilde{\mathrm{T}}_{i j k}} \Delta_{s} u_{h}(\mathrm{x}) v_{h}(\mathrm{x}) d s(\mathrm{x})=\int_{\widetilde{\mathrm{T}}_{i j k}} \nabla_{s} u_{h}(\mathrm{x}) \cdot \nabla_{s} v_{h}(\mathrm{x}) d s(\mathrm{x})-\int_{\partial \widetilde{\mathrm{T}}_{i j k}} \nabla_{s} u_{h}(\mathrm{x}) \cdot \overrightarrow{\mathrm{n}}_{\mathrm{x}, \widetilde{\mathrm{T}}_{i j k}} v_{h}(\mathrm{x}) d \gamma(\mathrm{x}) .
$$

Assim, da definição do funcional bilinear $\mathcal{A}(\cdot, \cdot)$, segue que

$$
\mathcal{A}\left(u_{h}, v_{h}\right)=\sum_{\widetilde{\mathrm{T}}_{i j k} \in \widetilde{\mathcal{T}}_{h}}\left[-\int_{\widetilde{\mathrm{T}}_{i j k}} \Delta_{s} u_{h}(\mathrm{x}) v_{h}(\mathrm{x}) d s(\mathrm{x})+\int_{\partial \widetilde{\mathrm{T}}_{i j k}} \nabla_{s} u_{h}(\mathrm{x}) \cdot \overrightarrow{\mathrm{n}}_{\mathrm{x}, \widetilde{\mathrm{T}}_{i j k}} v_{h}(\mathrm{x}) d \gamma(\mathrm{x})\right] .
$$

Conforme o Lema 3.3, consideraremos de novo as regiões poligonais esféricas $\widetilde{\mathrm{Q}}_{i}, \widetilde{\mathrm{Q}}_{j}$ e $\widetilde{\mathrm{Q}}_{k}$ formadas pela intersecção do triângulo esférico $\widetilde{T}_{i j k}$ com as células de Voronoï associadas a cada um dos vértices do triângulo, i.e., $\widetilde{\mathrm{Q}}_{n}=\widetilde{\mathrm{V}}_{n} \cap \widetilde{\mathrm{T}}_{i j k}$, onde $n \in\{i, j, k\}$, com fronteira

$$
\partial \widetilde{\mathrm{Q}}_{n}=\left(\partial \widetilde{\mathrm{V}}_{n} \cap \widetilde{\mathrm{T}}_{i j k}\right) \cup\left(\partial \widetilde{\mathrm{T}}_{i j k} \cap \widetilde{\mathrm{V}}_{n}\right), \quad \text { para } n \in\{i, j, k\} .
$$


Dada $\left.u_{h}\right|_{\widetilde{T}_{i j k}} \in H^{2}\left(\widetilde{\mathrm{T}}_{i j k}\right)$, multiplicamos $-\Delta_{s} u_{h}$, agora pelo termo $\mathrm{I}_{h}\left(v_{h}\right) \in \widetilde{V}_{h}$ e integrando em relação a $\widetilde{T}_{i j k}$, obteremos da fórmula de Green que

$$
\begin{aligned}
-\int_{\widetilde{\mathrm{T}}_{i j k}} \Delta_{s} u_{h}(\mathrm{x}) \mathrm{I}_{h}\left(v_{h}\right)(\mathrm{x}) d s(\mathrm{x})= & \sum_{n=i, j, k} \int_{\widetilde{\mathrm{Q}}_{n}} \nabla_{s} u_{h}(\mathrm{x}) \cdot \nabla_{s} \mathrm{I}_{h}\left(v_{h}\right)(\mathrm{x}) d s(\mathrm{x}) \\
& -\sum_{n=i, j, k} \int_{\partial \widetilde{\mathrm{Q}}_{n}} \nabla_{s} u_{h}(\mathrm{x}) \cdot \overrightarrow{\mathrm{n}}_{\mathrm{x}, \widetilde{\mathrm{Q}}_{n}} \mathrm{I}_{h}\left(v_{h}\right)(\mathrm{x}) d \gamma(\mathrm{x}) \\
= & -\sum_{n=i, j, k} \int_{\partial \widetilde{\mathrm{Q}}_{n}} \nabla_{s} u_{h}(\mathrm{x}) \cdot \overrightarrow{\mathrm{n}}_{\mathrm{x}, \widetilde{\mathrm{Q}}_{n}} \mathrm{I}_{h}\left(v_{h}\right)(\mathrm{x}) d \gamma(\mathrm{x}) .
\end{aligned}
$$

Reorganizando as fronteiras de $\widetilde{\mathrm{Q}}_{n}, n \in\{i, j, k\}$ segue,

$$
\begin{aligned}
-\int_{\widetilde{\mathrm{T}}_{i j k}} \Delta_{s} u_{h}(\mathrm{x}) \mathrm{I}_{h}\left(v_{h}\right)(\mathrm{x}) d s(\mathrm{x})= & -\int_{\partial \widetilde{\mathrm{T}}_{i j k}} \nabla_{s} u_{h}(\mathrm{x}) \cdot \overrightarrow{\mathrm{n}}_{\mathrm{x}, \widetilde{\mathrm{T}}_{i j k}} \mathrm{I}_{h}\left(v_{h}\right)(\mathrm{x}) d \gamma(\mathrm{x}) \\
& -\sum_{n=i, j, k} \int_{\partial \widetilde{\mathrm{V}}_{n} \cap \widetilde{\mathrm{T}}_{i j k}} \nabla_{s} u_{h}(\mathrm{x}) \cdot \overrightarrow{\mathrm{n}}_{\mathrm{x}, \widetilde{\mathrm{V}}_{n}} \mathrm{I}_{h}\left(v_{h}\right)(\mathrm{x}) d \gamma(\mathrm{x}) .
\end{aligned}
$$

Agora, somando em relação a todos os triângulos de $\widetilde{\mathcal{T}}_{h}$ e pelo princípio de dualidade, cada aresta do triângulo intersecta uma única aresta dual de Voronoï (vide Subseção 2.2.2), obtemos que

$$
\begin{aligned}
-\sum_{\widetilde{\mathrm{T}}_{i j k} \in \widetilde{\mathcal{T}}_{h}} \int_{\widetilde{\mathrm{T}}_{i j k}} \Delta_{s} u_{h}(\mathrm{x}) \mathrm{I}_{h}\left(v_{h}\right)(\mathrm{x}) d s(\mathrm{x})= & -\sum_{\widetilde{\mathrm{T}}_{i j k} \in \widetilde{\mathcal{T}}_{h}} \int_{\partial \widetilde{\mathrm{T}}_{i j k}} \nabla_{s} u_{h}(\mathrm{x}) \cdot \overrightarrow{\mathrm{n}}_{\mathrm{x}, \widetilde{\mathrm{T}}_{i j k}} \mathrm{I}_{h}\left(v_{h}\right)(\mathrm{x}) d \gamma(\mathrm{x}) \\
& -\sum_{i=1}^{N} \int_{\partial \widetilde{\mathrm{V}}_{i}} \nabla_{s} u_{h}(\mathrm{x}) \cdot \overrightarrow{\mathrm{n}}_{\mathrm{x}, \widetilde{\mathrm{V}}_{i}} \mathrm{I}_{h}\left(v_{h}\right)(\mathrm{x}) d \gamma(\mathrm{x}) .
\end{aligned}
$$

Observe-se que a última integral é a definição do funcional bilinear $\mathcal{A}_{h}(\cdot, \cdot)$, logo adotando essa notação, temos

$$
\mathcal{A}_{h}\left(u_{h}, \mathrm{I}_{h}\left(v_{h}\right)\right)=\sum_{\widetilde{\mathrm{T}}_{i j k} \in \widetilde{\mathcal{T}}_{h}}\left[-\int_{\widetilde{\mathrm{T}}_{i j k}} \Delta_{s} u_{h}(\mathrm{x}) \mathrm{I}_{h}\left(v_{h}\right)(\mathrm{x}) d s(\mathrm{x})+\int_{\partial \widetilde{\mathrm{T}}_{i j k}} \nabla_{s} u_{h}(\mathrm{x}) \cdot \overrightarrow{\mathrm{n}}_{\mathrm{x}, \widetilde{\mathrm{T}}_{i j k}} \mathrm{I}_{h}\left(v_{h}\right)(\mathrm{x}) d \gamma(\mathrm{x})\right] .
$$

Subtraindo os funcionais acima, resulta

$$
\begin{aligned}
\left|\mathcal{A}\left(u_{h}, v_{h}\right)-\mathcal{A}_{h}\left(u_{h}, \mathrm{I}_{h}\left(v_{h}\right)\right)\right| \leq & \left|\sum_{\widetilde{\mathrm{T}}_{i j k} \in \widetilde{\mathcal{T}}_{h}} \int_{\widetilde{\mathrm{T}}_{i j k}} \Delta_{s} u_{h}(\mathrm{x})\left[v_{h}(\mathrm{x})-\mathrm{I}_{h}\left(v_{h}\right)(\mathrm{x})\right] d s(\mathrm{x})\right| \\
& +\left|\sum_{\widetilde{\mathrm{T}}_{i j k} \in \widetilde{\mathcal{T}}_{h}} \int_{\partial \widetilde{\mathrm{T}}_{i j k}} \nabla_{s} u_{h}(\mathrm{x}) \cdot \overrightarrow{\mathrm{n}}_{\mathrm{x}, \widetilde{\mathrm{T}}_{i j k}}\left[v_{h}(\mathrm{x})-\mathrm{I}_{h}\left(v_{h}\right)(\mathrm{x})\right] d \gamma(\mathrm{x})\right| .
\end{aligned}
$$

Para cada aresta $\widetilde{\tau}_{i j}$ de $\widetilde{T}_{i j k}$, em virtude da simetria em relação ao ponto médio da aresta $\widetilde{\tau}_{i j}$, temos que o fluxo $\nabla_{s} u_{h}(\mathrm{x}) \cdot \overrightarrow{\mathrm{n}}_{\mathrm{x}, \widetilde{\mathrm{T}}_{i j k}}$ é uma função par para cada $\mathrm{x} \in \widetilde{\tau}_{i j}$. Segue do Lema 3.3 que

$$
\sum_{\widetilde{\mathrm{T}}_{i j k} \in \widetilde{\mathcal{T}}_{h}} \int_{\partial \widetilde{\mathrm{T}}_{i j k}} \nabla_{s} u_{h}(\mathrm{x}) \cdot \overrightarrow{\mathrm{n}}_{\mathrm{x}, \widetilde{\mathrm{T}}_{i j k}}\left[v_{h}(\mathrm{x})-\mathrm{I}_{h}\left(v_{h}\right)(\mathrm{x})\right] d \gamma(\mathrm{x})=0 .
$$


Em consequência temos,

$$
\left|\mathcal{A}\left(u_{h}, v_{h}\right)-\mathcal{A}_{h}\left(u_{h}, \mathrm{I}_{h}\left(v_{h}\right)\right)\right| \leq\left|\sum_{\widetilde{\mathrm{T}}_{i j k} \in \widetilde{\mathcal{T}}_{h}} \int_{\widetilde{\mathrm{T}}_{i j k}} \Delta_{s} u_{h}(\mathrm{x})\left[v_{h}(\mathrm{x})-\mathrm{I}_{h}\left(v_{h}\right)(\mathrm{x})\right] d s(\mathrm{x})\right| .
$$

Para continuar a demonstração, vamos considerar as extensões $\bar{u}_{h}^{\Omega}$ e $\bar{v}_{h}^{\Omega}$ restritas ao poliedro $\mathbf{S}_{h}$ de $u_{h}$ e $v_{h}$ respectivamente. Resulta imediatamente que $\bar{u}_{h}^{\Omega}$ é harmônica em cada triângulo plano $\mathrm{T}_{i j k} \in$ $\mathcal{T}_{h}$, i.e., $\Delta \bar{u}^{\Omega}\left(\mathrm{x}^{*}\right)=0$ para cada $\mathrm{x}^{*} \in \mathrm{T}_{i j k}$. Sabemos que $v_{h}(\mathrm{x})=\bar{v}_{h}^{\Omega}\left(\mathrm{x}^{*}\right)$ para cada $\mathrm{x}=\mathcal{P}\left(\mathrm{x}^{*}\right)$ e como os vértices de $\mathbf{S}_{h}$ estão situados em $\mathbb{S}^{2}$, temos a relação $\mathrm{I}_{h}\left(v_{h}\right)(\mathrm{x})=\mathrm{I}_{h}\left(v_{h}^{\Omega}\right)\left(\mathcal{P}^{-1}(\mathrm{x})\right)=\mathrm{I}_{h}\left(\bar{v}_{h}^{\Omega}\right)\left(\mathrm{x}^{*}\right)$ para cada $\mathrm{x}^{*} \in \mathrm{T}_{i j k}$. Agora, se multiplicamos $\Delta \bar{u}_{h}^{\Omega}$ por $\bar{v}_{h}^{\Omega}-\mathrm{I}_{h}\left(\bar{v}_{h}^{\Omega}\right)$ e integrando em relação a $\mathrm{T}_{i j k}$, resulta

$$
\int_{\mathrm{T}_{i j k}} \Delta \bar{u}_{h}^{\Omega}\left(\mathrm{x}^{*}\right)\left[\bar{v}_{h}^{\Omega}\left(\mathrm{x}^{*}\right)-\mathrm{I}_{h}\left(\bar{v}_{h}^{\Omega}\right)\left(\mathrm{x}^{*}\right)\right] d s\left(\mathrm{x}^{*}\right)=0 .
$$

Levando em mente o anterior junto à mudança de variável com $\left|\left\|\mathrm{x}^{*}\right\|-1\right| \leq C h^{2}$, resulta

$$
\begin{aligned}
\left|\mathcal{A}\left(u_{h}, v_{h}\right)-\mathcal{A}_{h}\left(u_{h}, \mathrm{I}_{h}\left(v_{h}\right)\right)\right|= & \left|\sum_{\widetilde{\mathrm{T}}_{i j k} \in \widetilde{\mathcal{T}}_{h}} \int_{\widetilde{\mathrm{T}}_{i j k}} \Delta_{s} u_{h}(\mathrm{x})\left[v_{h}(\mathrm{x})-\mathrm{I}_{h}\left(v_{h}\right)(\mathrm{x})\right] d s(\mathrm{x})\right| \\
= & \mid \sum_{\mathrm{T}_{i j k} \in \mathcal{T}_{h}}\left(\int_{\mathrm{T}_{i j k}}\left\|\mathrm{x}^{*}\right\| \Delta \bar{u}_{h}^{\Omega}\left(\mathrm{x}^{*}\right)\left[\bar{v}_{h}^{\Omega}\left(\mathrm{x}^{*}\right)-\mathrm{I}_{h}\left(\bar{v}_{h}^{\Omega}\right)\left(\mathrm{x}^{*}\right)\right] d s\left(\mathrm{x}^{*}\right)\right. \\
& \left.-\int_{\mathrm{T}_{i j k}} \Delta \bar{u}_{h}^{\Omega}\left(\mathrm{x}^{*}\right)\left[\bar{v}_{h}^{\Omega}\left(\mathrm{x}^{*}\right)-\mathrm{I}_{h}\left(\bar{v}_{h}^{\Omega}\right)\left(\mathrm{x}^{*}\right)\right] d s\left(\mathrm{x}^{*}\right)\right) \mid \\
\leq & \sum_{\mathrm{T}_{i j k} \in \mathcal{T}_{h}} \int_{\mathrm{T}_{i j k}}\left|\left\|\mathrm{x}^{*}\right\|-1\right|\left|\Delta \bar{u}_{h}^{\Omega}\left(\mathrm{x}^{*}\right)\left[\bar{v}_{h}^{\Omega}\left(\mathrm{x}^{*}\right)-\mathrm{I}_{h}\left(\bar{v}_{h}^{\Omega}\right)\left(\mathrm{x}^{*}\right)\right]\right| d s\left(\mathrm{x}^{*}\right) \\
\leq & C h^{2} \sum_{\mathrm{T}_{i j k} \in \mathcal{T}_{h}} \int_{\mathrm{T}_{i j k}}\left|\Delta \bar{u}_{h}^{\Omega}\left(\mathrm{x}^{*}\right)\left[\bar{v}_{h}^{\Omega}\left(\mathrm{x}^{*}\right)-\mathrm{I}_{h}\left(\bar{v}_{h}^{\Omega}\right)\left(\mathrm{x}^{*}\right)\right]\right| d s\left(\mathrm{x}^{*}\right) .
\end{aligned}
$$

Por último, invocando a desigualdade de Cauchy-Schwarz, a Proposição 3.9 e combinando os Lemas 3.3 e 3.2 , temos

$$
\begin{aligned}
\left|\mathcal{A}\left(u_{h}, v_{h}\right)-\mathcal{A}_{h}\left(u_{h}, \mathrm{I}_{h}\left(v_{h}\right)\right)\right| \leq & C h^{2} \sum_{\mathrm{T}_{i j k} \in \mathcal{T}_{h}}\left(\int_{\mathrm{T}_{i j k}}\left|\Delta \bar{u}_{h}^{\Omega}\left(\mathrm{x}^{*}\right)\right|^{2} d s\left(\mathrm{x}^{*}\right)\right)^{1 / 2} \\
& \times\left(\int_{\mathrm{T}_{i j k}}\left|\bar{v}_{h}^{\Omega}\left(\mathrm{x}^{*}\right)-\mathrm{I}_{h}\left(\bar{v}_{h}^{\Omega}\right)\left(\mathrm{x}^{*}\right)\right|^{2} d s\left(\mathrm{x}^{*}\right)\right)^{1 / 2} \\
\leq & C h^{2} \sum_{\leq}\left\|\bar{u}_{h}^{\Omega}\right\|_{H^{2}\left(\mathrm{~T}_{i j k}\right)}\left\|\bar{v}_{h}^{\Omega}-\mathrm{I}_{h}\left(\bar{v}_{h}^{\Omega}\right)\right\|_{L^{2}\left(\mathrm{~T}_{i j k}\right)} \\
\leq & C h^{2} \sum_{\widetilde{\mathrm{T}}_{i j k} \in \widetilde{\mathcal{T}}_{h}}\left\|u_{h}\right\|_{H^{2}\left(\widetilde{\mathrm{T}}_{i j k}\right)}\left\|v_{h}-\mathrm{I}_{h}\left(v_{h}\right)\right\|_{L^{2}\left(\widetilde{\mathrm{T}}_{i j k}\right)} \\
\leq & C h^{2} \sum_{\widetilde{\mathrm{T}}_{i j k} \in \widetilde{\mathcal{T}}_{h}}\left\|u_{h}\right\|_{H^{1}\left(\widetilde{\mathrm{T}}_{i j k}\right)}\left\|v_{h}\right\|_{H^{1}\left(\widetilde{\mathrm{T}}_{i j k}\right)} \\
\leq & C h^{2}\left\|u_{h}\right\|_{H^{1}\left(\mathbb{S}^{2}\right)}\left\|v_{h}\right\|_{H^{1}\left(\mathbb{S}^{2}\right)} .
\end{aligned}
$$

O que provaria (3.29). Isto encerra a demostração.

Observação 3.7. Similar ao Lema 3.4, com mudanças menores o lema acima é válido para cada 
$u_{h}, v_{h} \in \widetilde{U}_{h}$, com $p \geq 1,1 / p+1 / q=1$, tem-se pela desigualdade de Hölder a estimativa

$$
\left|\mathcal{A}\left(u_{h}, \mathrm{I}_{h}\left(v_{h}\right)\right)-\mathcal{A}_{h}\left(u_{h}, \mathrm{I}_{h}\left(v_{h}\right)\right)\right| \leq C h^{2}\left\|\nabla_{s} u_{h}\right\|_{L^{p}\left(\mathbb{S}^{2}\right)}\left\|\nabla_{s} v_{h}\right\|_{L^{q}\left(\mathbb{S}^{2}\right)}, \quad \text { para cada } v_{h} \in \widetilde{U}_{h} .
$$

Observação 3.8. Deve-se notar que para problemas no plano a diferença (3.29) é identicamente zero, dado que a função linear por pedaços é harmônica em cada triângulo e além disso a dualidade entre as decomposições primal e dual, implicariam, que as formas bilineares tanto do MVF como do MEFL, são equivalentes como descrito por Bank e Rose (1987); Hackbusch (1989); Vanselow (1996). Em particular, dada a função $u_{h}(\mathrm{x})=x_{1}$, com $\mathrm{x}=\left(x_{1}, x_{2}, x_{3}\right) \in \mathbb{S}^{2}$, temos que $\Delta_{s} u_{h} \neq 0$, ainda quando $u_{h}$ é harmônica no $\mathbb{R}^{3}$. 


\section{Capítulo 4}

\section{Estimativas a priori do erro}

Neste capítulo apresentaremos os resultados principais desta tese, a saber a estimativa da ordem de convergência das soluções do Laplaciano via o MFV nas normas $H^{1}, L^{2}$, max-norma e $W^{1, \infty}$ norma para as malhas esféricas icosaédricas. Estabeleceremos as estimativas usando a estrutura de Du e Ju (2005); Ewing et al. (2002); Ju e Du (2009), para a decomposição Voronoï-Delaunay em $\mathbb{S}^{2}$. Ressaltamos que nas estimativas abaixo interfere a posição dos vértices das malhas duais (os circuncentros), logo a configuração geométrica desses influi na ordem de convergência. Começamos com o seguinte lema:

Lema 4.1. Assuma que $f \in L^{p}\left(\mathbb{S}^{2}\right)$, com $p \geq 1$ que satisfaz a condição de compatibilidade (1.3). Então, para cada $v_{h} \in \widetilde{U}_{h}, 1 / p+1 / q=1$. Existe uma constante $C \in \mathbb{R}_{+}$, que independe de $h$, tal que

$$
\left|\left(f, v_{h}\right)-\left\langle f_{h}, \mathrm{I}_{h}\left(v_{h}\right)\right\rangle_{h}\right| \leq C h\|f\|_{L^{p}\left(\mathbb{S}^{2}\right)}\left\|v_{h}\right\|_{W^{1, q}\left(\mathbb{S}^{2}\right)},
$$

Demonstração. Com efeito, dada $f_{h}$ a aproximação de $f$, o produto escalar produz $\left\langle f_{h}, \mathrm{I}_{h}\left(v_{h}\right)\right\rangle_{h}=$ $\left(f, \mathrm{I}_{h}\left(v_{h}\right)\right)$. Então,

$$
\begin{aligned}
\left|\left(f, v_{h}\right)-\left(f, \mathrm{I}_{h}\left(v_{h}\right)\right)\right|=\left|\left(f, v_{h}-\mathrm{I}_{h}\left(v_{h}\right)\right)\right| & =\left|\int_{\mathbb{S}^{2}} f(\mathrm{x})\left[v_{h}(\mathrm{x})-\mathrm{I}_{h}\left(v_{h}\right)(\mathrm{x})\right] d s(\mathrm{x})\right| \\
& =\left|\sum_{\widetilde{\mathrm{T}}_{i j k} \in \widetilde{\mathcal{T}}_{h}} \int_{\widetilde{\mathrm{T}}_{i j k}} f(\mathrm{x})\left[v_{h}(\mathrm{x})-\mathrm{I}_{h}\left(v_{h}\right)(\mathrm{x})\right] d s(\mathrm{x})\right| .
\end{aligned}
$$

Aplicando a desigualdade de Hölder com $1 / p+1 / q=1$ e em seguida o Lema 3.3, obtemos

$$
\begin{aligned}
\left|\left(f, v_{h}\right)-\left(f, \mathrm{I}_{h}\left(v_{h}\right)\right)\right| & =\left|\sum_{\widetilde{\mathrm{T}}_{i j k} \in \widetilde{\mathcal{T}}_{h}} \int_{\widetilde{\mathrm{T}}_{i j k} \in \widetilde{\mathcal{T}}_{h}} f(\mathrm{x})\left[v_{h}(\mathrm{x})-\mathrm{I}_{h}\left(v_{h}\right)(\mathrm{x})\right] d s(\mathrm{x})\right| \\
& \leq C h \sum_{\widetilde{\mathrm{T}}_{i j k} \in \widetilde{\mathcal{T}}_{h}}\|f\|_{L^{p}\left(\widetilde{\mathrm{T}}_{i j k}\right)}\left\|v_{h}\right\|_{W^{1, q}\left(\widetilde{\mathrm{T}}_{i j k}\right)} \\
& \leq C h\|f\|_{L^{p}\left(\mathbb{S}^{2}\right)}\left\|v_{h}\right\|_{W^{1, q}\left(\mathbb{S}^{2}\right)} .
\end{aligned}
$$

Portanto, segue a limitação (4.1).

\subsection{Estimativas clássicas na $H^{1}$ e $L^{2}$-norma}

Consideraremos inicialmente o erro das soluções na $H^{1}$-norma. Em geral, a estimativa obtida de ordem um para problemas no plano foi exposta por Gallouët et al. (2000); Herbin (1995); Mishev (1998) e Eymard et al. (2001) usando a limitação do erro de consistência na $L^{2}$-norma. Essa ideia foi estendida para a esfera por Du et al. (2003b) para a $H^{1}$-norma discreta. Nesse contexto, a prova de convergência descrita abaixo, cuja principal ideia foi motivada por Ewing et al. (2002), difere dos trabalhos mencionados, dado que usa os procedimentos padrão das estimativas de erro para os MEFL. Em outras palavras, a característica essencial centra-se nos Lemas 3.4-4.1 junto com a Proposição 3.10. Por outro lado, sua relevância estará presente nos Teoremas 4.2-4.6. 
Teorema 4.1. Sejam $\widetilde{\mathcal{V}}=\left\{\mathrm{x}_{i}, \widetilde{\mathrm{V}}_{i}\right\}_{i=1}^{N}$ a decomposição Voronoï-Delaunay (NOPT) para $\mathbb{S}^{2}$ que satisfaz as condições (2.5a)-(2.5c) e $f \in L^{2}\left(\mathbb{S}^{2}\right)$ que satisfaz a condição de compatibilidade (1.3). Assuma que a única solução fraca u de (1.8) pertence ao espaço $H^{2}\left(\mathbb{S}^{2}\right) \cap H_{Q}^{1}\left(\mathbb{S}^{2}\right)$. Seja $\mathrm{F}_{i j}^{d}$ o fluxo discreto definido em (3.8), tal que o problema discreto (3.23) possui única solução $u_{h} \in \widetilde{U}_{h}$. Então, existe uma constante $C \in \mathbb{R}_{+}$, independente de $h$, tal que

$$
\left\|\varepsilon_{h}\right\|_{H^{1}\left(\mathbb{S}^{2}\right)} \leq C h\left(\|u\|_{H^{2}\left(\mathbb{S}^{2}\right)}+\|f\|_{L^{2}\left(\mathbb{S}^{2}\right)}\right)
$$

onde $\varepsilon_{h}=u-u_{h}$.

Demonstração. Para a prova, vamos considerar a coercividade da forma variacional $\mathcal{A}(\cdot, \cdot)$. Em seguida, da Proposição 3.10 , somaremos e subtrairemos a interpolação de $u$ a $\widetilde{U}_{h}$, i.e., o termo $\Pi_{h}(u)$, e usando a desigualdade triangular resulta a expressão

$$
\begin{aligned}
\left\|u-u_{h}\right\|_{H^{1}\left(\mathbb{S}^{2}\right)}^{2} & \preceq\left|\mathcal{A}\left(u-u_{h}, u-u_{h}\right)\right| \\
& \preceq\left|\mathcal{A}\left(u-u_{h}, u-\Pi_{h}(u)\right)\right|+\left|\mathcal{A}\left(u-u_{h}, \Pi_{h}(u)-u_{h}\right)\right| \\
& \preceq\left|\mathcal{A}\left(u-u_{h}, u-\Pi_{h}(u)\right)\right|+\left|\mathcal{A}\left(u, \Pi_{h}(u)-u_{h}\right)-\mathcal{A}\left(u_{h}, \Pi_{h}(u)-u_{h}\right)\right|,
\end{aligned}
$$

onde a constante oculta em " $\preceq$ " vem da coercividade de $\mathcal{A}(\cdot, \cdot)$. Agora, somando e subtraindo os funcionais $\mathcal{A}_{h}^{d}(\cdot, \cdot), \mathcal{A}_{h}(\cdot, \cdot)$ e usando uma vez mais a desigualdade triangular produzimos a expressão,

$$
\begin{aligned}
\left\|u-u_{h}\right\|_{H^{1}\left(\mathbb{S}^{2}\right)}^{2} \preceq & \left|\mathcal{A}\left(u-u_{h}, u-\Pi_{h}(u)\right)\right|+\left|\mathcal{A}\left(u, \Pi_{h}(u)-u_{h}\right)-\mathcal{A}_{h}^{d}\left(u_{h}, \mathrm{I}_{h}\left(\Pi_{h}(u)-u_{h}\right)\right)\right| \\
& +\left|\mathcal{A}_{h}^{d}\left(u_{h}, \mathrm{I}_{h}\left(\Pi_{h}(u)-u_{h}\right)\right)-\mathcal{A}_{h}\left(u_{h}, \mathrm{I}_{h}\left(\Pi_{h}(u)-u_{h}\right)\right)\right| \\
& +\left|\mathcal{A}_{h}\left(u_{h}, \mathrm{I}_{h}\left(\Pi_{h}(u)-u_{h}\right)\right)-\mathcal{A}\left(u_{h}, \Pi_{h}(u)-u_{h}\right)\right| . \\
= & I_{1}+I_{2}+I_{3}+I_{4},
\end{aligned}
$$

onde

$$
\begin{aligned}
& I_{1}=\left|\mathcal{A}\left(u-u_{h}, u-\Pi_{h}(u)\right)\right|, \\
& I_{2}=\left|\mathcal{A}\left(u, \Pi_{h}(u)-u_{h}\right)-\mathcal{A}_{h}^{d}\left(u_{h}, \mathrm{I}_{h}\left(\Pi_{h}(u)-u_{h}\right)\right)\right|, \\
& I_{3}=\left|\mathcal{A}_{h}^{d}\left(u_{h}, \mathrm{I}_{h}\left(\Pi_{h}(u)-u_{h}\right)\right)-\mathcal{A}_{h}\left(u_{h}, \mathrm{I}_{h}\left(\Pi_{h}(u)-u_{h}\right)\right)\right|, \\
& I_{4}=\left|\mathcal{A}_{h}\left(u_{h}, \mathrm{I}_{h}\left(\Pi_{h}(u)-u_{h}\right)\right)-\mathcal{A}\left(u_{h}, \Pi_{h}(u)-u_{h}\right)\right| .
\end{aligned}
$$

Para $I_{1}$, da continuidade de $\mathcal{A}(\cdot, \cdot)$ e da Proposição 3.10 segue que

$$
\begin{aligned}
I_{1}=\left|\mathcal{A}\left(u-u_{h}, u-\Pi_{h}(u)\right)\right| & \leq C\left\|u-u_{h}\right\|_{H^{1}\left(\mathbb{S}^{2}\right)}\left\|u-\Pi_{h}(u)\right\|_{H^{1}\left(\mathbb{S}^{2}\right)} \\
& \leq C h\left\|u-u_{h}\right\|_{H^{1}\left(\mathbb{S}^{2}\right)}\|u\|_{H^{2}\left(\mathbb{S}^{2}\right)} .
\end{aligned}
$$

Dos problemas variacionais $(1.8)$ e (3.23) temos para $I_{2}$

$$
I_{2}=\left|\mathcal{A}\left(u, \Pi_{h}(u)-u_{h}\right)-\mathcal{A}_{h}^{d}\left(u_{h}, \mathrm{I}_{h}\left(\Pi_{h}(u)-u_{h}\right)\right)\right|=\left|\left(f, \Pi_{h}(u)-u_{h}\right)-\left\langle f_{h}, \mathrm{I}_{h}\left(\Pi_{h}(u)-u_{h}\right)\right\rangle_{h}\right| .
$$

Então, pondo $p=q=2$ no Lema 4.1 produz para $\Pi_{h}(u)-u_{h} \in \widetilde{U}_{h}$,

$$
I_{2}=\left|\left(f, \Pi_{h}(u)-u_{h}-\mathrm{I}_{h}\left(\Pi_{h}(u)-u_{h}\right)\right)\right| \leq C h\|f\|_{L^{2}\left(\mathbb{S}^{2}\right)}\left\|\Pi_{h}(u)-u_{h}\right\|_{H^{1}\left(\mathbb{S}^{2}\right)} .
$$

Do Lema 3.4 obtemos para $I_{3}$,

$$
I_{3}=\left|\mathcal{A}_{h}^{d}\left(u_{h}, \mathrm{I}_{h}\left(\Pi_{h}(u)-u_{h}\right)\right)-\mathcal{A}_{h}\left(u_{h}, \mathrm{I}_{h}\left(\Pi_{h}(u)-u_{h}\right)\right)\right| \leq C h^{2}\left\|u_{h}\right\|_{H^{1}\left(\mathbb{S}^{2}\right)}\left\|\Pi_{h}(u)-u_{h}\right\|_{H^{1}\left(\mathbb{S}^{2}\right)},
$$


e para $I_{4}$, invocando o Lema 3.5 geramos a estimativa

$$
I_{4}=\left|\mathcal{A}_{h}\left(u_{h}, \mathrm{I}_{h}\left(\Pi_{h}(u)-u_{h}\right)\right)-\mathcal{A}\left(u_{h}, \Pi_{h}(u)-u_{h}\right)\right| \leq C h^{2}\left\|u_{h}\right\|_{H^{1}\left(\mathbb{S}^{2}\right)}\left\|\Pi_{h}(u)-u_{h}\right\|_{H^{1}\left(\mathbb{S}^{2}\right)} .
$$

Da condição de estabilidade (3.25) e a equivalência das normas, Proposição 3.11, segue que

$$
\left\|u_{h}\right\|_{H^{1}\left(\mathbb{S}^{2}\right)} \leq C\|f\|_{L^{2}\left(\mathbb{S}^{2}\right)} .
$$

Invocando uma vez mais a Proposição 3.10, obteremos

$$
\left\|\Pi_{h}(u)-u_{h}\right\|_{H^{1}\left(\mathbb{S}^{2}\right)} \leq\left\|u-\Pi_{h}(u)\right\|_{H^{1}\left(\mathbb{S}^{2}\right)}+\left\|u-u_{h}\right\|_{H^{1}\left(\mathbb{S}^{2}\right)}\|\leq C h\| u\left\|_{H^{2}\left(\mathbb{S}^{2}\right)}+\right\| u-u_{h} \|_{H^{1}\left(\mathbb{S}^{2}\right)} .
$$

Então, para $h>0$ suficientemente pequeno, temos para $I_{2}, I_{3}$ e $I_{4}$ que

$$
\begin{aligned}
I_{2} & \leq C h\|f\|_{L^{2}\left(\mathbb{S}^{2}\right)}\left\|u-u_{h}\right\|_{H^{1}\left(\mathbb{S}^{2}\right)}, \\
I_{3}, I_{4} & \leq C h^{2}\|f\|_{L^{2}\left(\mathbb{S}^{2}\right)}\left\|u-u_{h}\right\|_{H^{1}\left(\mathbb{S}^{2}\right)} .
\end{aligned}
$$

Combinando (4.3)-(4.8), junto com (4.2), resulta

$$
\left\|u-u_{h}\right\|_{H^{1}\left(\mathbb{S}^{2}\right)}^{2} \leq C h\left\|u-u_{h}\right\|_{H^{1}\left(\mathbb{S}^{2}\right)}\left(\|u\|_{H^{2}\left(\mathbb{S}^{2}\right)}+\|f\|_{L^{2}\left(\mathbb{S}^{2}\right)}\right) .
$$

Dividindo por $\left\|u-u_{h}\right\|_{H^{1}\left(\mathbb{S}^{2}\right)}$ cada lado da expressão acima obtemos o resultado desejado.

A seguinte estimativa de ordem um na $L^{2}$-norma é válida para decomposições Voronoï-Delaunay em geral. A mesma ordem de precisão foi obtida para problemas no plano por Gallouët et al. (2000) e Eymard et al. (2000) e para malhas não estruturadas por Coudière et al. (1999) e Eymard et al. (2006). O procedimento nesses trabalhos foi usar uma versão discreta da desigualdade de Poincaré-Friedrich junto à estimativa na $H^{1}$-norma. Evidentemente nesses resultados, a ordem de convergência depende novamente do erro de consistência na $L^{2}$-norma. A prova abaixo é deduzida usando um argumento de dualidade aliado ao Teorema 4.1.

Teorema 4.2. Sob as hipóteses do Teorema 4.1. Existe uma constante $C \in \mathbb{R}_{+}$, independente de $h$, tal que

$$
\left\|\varepsilon_{h}\right\|_{L^{2}\left(\mathbb{S}^{2}\right)} \leq C h\|f\|_{L^{2}\left(\mathbb{S}^{2}\right)}
$$

onde $\varepsilon_{h}=u-u_{h}$.

Demonstração. Para a prova usamos o seguinte argumento de dualidade: como $u-u_{h} \in H_{Q}^{1}\left(\mathbb{S}^{2}\right)$, sabemos de (1.8) que existe uma única solução fraca $w \in H^{2}\left(\mathbb{S}^{2}\right) \cap H_{Q}^{1}\left(\mathbb{S}^{2}\right)$ que satisfaz o problema variacional adjunto:

$$
\mathcal{A}(v, w)=\left(v, u-u_{h}\right) \quad \text { para cada } v \in H_{Q}^{1}\left(\mathbb{S}^{2}\right) .
$$

Pondo $v=u-u_{h}$, para $w$ segue da condição de regularidade (1.9) que

$$
\|w\|_{H^{2}\left(\mathbb{S}^{2}\right)} \leq C\left\|u-u_{h}\right\|_{L^{2}\left(\mathbb{S}^{2}\right)} .
$$

Agora, assumindo $w_{h}=\Pi_{h}(w) \in \widetilde{U}_{h}$ e usando um argumento similar ao Teorema 4.1, temos que

$$
\begin{aligned}
\left\|u-u_{h}\right\|_{L^{2}\left(\mathbb{S}^{2}\right)}^{2}= & \left(u-u_{h}, u-u_{h}\right) \leq\left|\mathcal{A}\left(u-u_{h}, w\right)\right| \\
= & \left|\mathcal{A}\left(u-u_{h}, w-w_{h}\right)+\mathcal{A}\left(u-u_{h}, w_{h}\right)\right| \\
= & \left|\mathcal{A}\left(u-u_{h}, w-w_{h}\right)+\mathcal{A}\left(u, w_{h}\right)-\mathcal{A}\left(u_{h}, w_{h}\right)\right| \\
\leq & \left|\mathcal{A}\left(u-u_{h}, w-w_{h}\right)\right|+\left|\mathcal{A}\left(u, w_{h}\right)-\mathcal{A}_{h}^{d}\left(u_{h}, \mathrm{I}_{h}\left(w_{h}\right)\right)\right| \\
& +\left|\mathcal{A}_{h}^{d}\left(u_{h}, \mathrm{I}_{h}\left(w_{h}\right)\right)-\mathcal{A}_{h}\left(u_{h}, \mathrm{I}_{h}\left(w_{h}\right)\right)\right|+\left|\mathcal{A}_{h}\left(u_{h}, \mathrm{I}_{h}\left(w_{h}\right)\right)-\mathcal{A}\left(u_{h}, w_{h}\right)\right| \\
= & I_{1}+I_{2}+I_{3}+I_{4},
\end{aligned}
$$


onde

$$
\begin{aligned}
I_{1} & =\left|\mathcal{A}\left(u-u_{h}, w-w_{h}\right)\right|, \\
I_{2} & =\left|\mathcal{A}\left(u, w_{h}\right)-\mathcal{A}_{h}^{d}\left(u, \mathrm{I}_{h}\left(w_{h}\right)\right)\right|, \\
I_{3} & =\left|\mathcal{A}_{h}^{d}\left(u_{h}, \mathrm{I}_{h}\left(w_{h}\right)\right)-\mathcal{A}_{h}\left(u_{h}, \mathrm{I}_{h}\left(w_{h}\right)\right)\right|, \\
I_{4} & =\left|\mathcal{A}_{h}\left(u_{h}, \mathrm{I}_{h}\left(w_{h}\right)\right)-\mathcal{A}\left(u_{h}, w_{h}\right)\right| .
\end{aligned}
$$

Do Teorema 4.1, a Proposição 3.10 e a limitação (4.9) obtemos para $I_{1}$ que

$$
\begin{aligned}
I_{1}=\left|\mathcal{A}\left(u-u_{h}, w-w_{h}\right)\right| & \leq C\left\|u-u_{h}\right\|_{H^{1}\left(\mathbb{S}^{2}\right)}\left\|w-w_{h}\right\|_{H^{1}\left(\mathbb{S}^{2}\right)} \\
& \leq C h^{2}\left(\|u\|_{H^{2}\left(\mathbb{S}^{2}\right)}+\|f\|_{L^{2}\left(\mathbb{S}^{2}\right)}\right)\|w\|_{H^{2}\left(\mathbb{S}^{2}\right)} \\
& \leq C h^{2}\left(\|u\|_{H^{2}\left(\mathbb{S}^{2}\right)}+\|f\|_{L^{2}\left(\mathbb{S}^{2}\right)}\right)\left\|u-u_{h}\right\|_{L^{2}\left(\mathbb{S}^{2}\right)} .
\end{aligned}
$$

Do Lema 4.1, da definição da $w_{h}$ e junto à estimativa (4.9) temos para $I_{2}$,

$$
\begin{aligned}
I_{2}=\left|\mathcal{A}\left(u, w_{h}\right)-\mathcal{A}_{h}\left(u, \mathrm{I}_{h}\left(w_{h}\right)\right)\right|=\left|\left(f, w_{h}-\mathrm{I}_{h}\left(w_{h}\right)\right)\right| & \leq C h\|f\|_{L^{2}\left(\mathbb{S}^{2}\right)}\left\|w_{h}\right\|_{H^{1}\left(\mathbb{S}^{2}\right)} \\
& \leq C h\|f\|_{L^{2}\left(\mathbb{S}^{2}\right)}\left\|u-u_{h}\right\|_{L^{2}\left(\mathbb{S}^{2}\right)},
\end{aligned}
$$

De forma análoga ao Teorema 4.1, usando o Lema 3.4, a estabilidade (3.25) e a estimativa (4.9) segue para $I_{3}$ que

$$
\begin{aligned}
I_{3}=\left|\mathcal{A}_{h}^{d}\left(u_{h}, \mathrm{I}_{h}\left(w_{h}\right)\right)-\mathcal{A}_{h}\left(u_{h}, \mathrm{I}_{h}\left(w_{h}\right)\right)\right| & \leq C h^{2}\|f\|_{L^{2}\left(\mathbb{S}^{2}\right)}\left\|w_{h}\right\|_{H^{1}\left(\mathbb{S}^{2}\right)} \\
& \leq C h^{2}\|f\|_{L^{2}\left(\mathbb{S}^{2}\right)}\left\|u-u_{h}\right\|_{L^{2}\left(\mathbb{S}^{2}\right)} .
\end{aligned}
$$

Por último, o Lema 3.5, a estabilidade (3.25) e (4.9) produzem a expressão

$$
I_{4} \leq C h^{2}\|f\|_{L^{2}\left(\mathbb{S}^{2}\right)}\left\|u-u_{h}\right\|_{L^{2}\left(\mathbb{S}^{2}\right)} .
$$

Combinando (4.10)-(4.13), obtemos para $h>0$ suficientemente pequeno que

$$
\left\|u-u_{h}\right\|_{L^{2}\left(\mathbb{S}^{2}\right)}^{2} \leq C h\|f\|_{L^{2}\left(\mathbb{S}^{2}\right)}\left\|u-u_{h}\right\|_{L^{2}\left(\mathbb{S}^{2}\right)} .
$$

Dividindo por $\left\|u-u_{h}\right\|_{L^{2}\left(\mathbb{S}^{2}\right)}$ cada lado da expressão encerraria a demonstração do Teorema.

Observação 4.1. Na estimativa foram impostas condições mínimas na regularidade da solução exata e no termo fonte. Porém, não parece relevante aumentarmos a regularidade em algum dos dois casos, para obter um ganho na ordem de convergência. Além disso, a taxa de convergência obtida é baixa em contraste ás aproximações bem compreendidas dos MEFL e como veremos nos experimentos numéricos, os erros das soluções dos MVF comportam-se melhor do que mostraram as provas anteriores. Aparentemente, a perda de ordem tem sido relacionada as propriedades geométricas da decomposição Voronö̈-Delaunay em geral, e por enquanto não existe um artifício padrão para incrementar a ordem de convergência na $L^{2}$-norma.

Deve-se notar que o termo dominante na limitação acima é dada pelo Lema 4.1. Então, uma forma de ganhar uma potencia "extra" na ordem de convergência, é usar a otimização do tipo SCVT na decomposição Voronoï-Delaunay como descrita na Subseção 2.3.1. Essa estimativa quadrática na $L^{2}$-norma já foi relatada por $\mathrm{Du}$ e Ju (2005), mas vamos ilustrar aqui visando a diminuição da regularidade na solução exata como relatado por Ewing et al. (2002). Para fim, precisamos enunciar uma aproximação em relação ao interpolador linear $\widetilde{\Pi}_{h}$. Mais precisamente:

Proposição 4.1 (Du e Ju, 2005). Para cada $w \in W^{2, q}\left(\mathbb{S}^{2}\right)$ e $1 \leq q<\infty$, tem-se

$$
\left\|w-\widetilde{\Pi}_{h}(w)\right\|_{L^{q}\left(\mathbb{S}^{2}\right)} \leq C h\|w\|_{W^{2, q}\left(\mathbb{S}^{2}\right)} .
$$


O próximo lema é uma versão mais simples do expresso por Du e Ju (2005, Lema 1, pp. 1682). Assumimos que a função de densidade $\rho$ é constante igual a 1.

Lema 4.2 (Du e Ju, 2005). Suponha que $\tilde{\mathcal{V}}=\left\{\mathrm{x}_{i}, \widetilde{\mathrm{V}}_{i}\right\}_{i=1}^{N}$ é uma decomposição dual de Voronö̈ do tipo $S C V T$ de $\mathbb{S}^{2}$ que satisfaz as condições (2.5a)-(2.5c). Então, para qualquer $w \in H^{2}\left(\mathbb{S}^{2}\right) \cap H_{Q}^{1}\left(\mathbb{S}^{2}\right)$, existe uma constante $C \in \mathbb{R}_{+}$, independente de $h$, tal que

$$
\left|\int_{\widetilde{\mathrm{V}}_{i}}\left[w(\mathrm{x})-\widetilde{\Pi}_{h}(w)(\mathrm{x})\right] d s(\mathrm{x})\right| \leq C h^{2} S\left(\widetilde{\mathrm{V}}_{i}\right)^{1 / 2}\|w\|_{H^{2}\left(\widetilde{\mathrm{V}}_{i}\right)} .
$$

Demonstração. Para mostrar a limitação é suficiente assumir que $\left.w(\mathrm{x})\right|_{\widetilde{\mathrm{V}}_{i}} \in C^{2}\left(\widetilde{\mathrm{V}}_{i}\right)$ e após invocar um argumento de densidade. Inicialmente temos que $\widetilde{\Pi}_{h}(w)(\mathrm{x})=w\left(\mathrm{x}_{i}\right)$ para cada $\mathrm{x} \in \widetilde{\mathrm{V}}_{i}$ e consideremos $w^{\Omega}$ a extensão de $w$ a $\Omega_{h}$. Pela construção, podemos usar o Teorema de Taylor até ordem dois de $w^{\Omega}$ em torno de x e integrando em relação à célula $\widetilde{\mathrm{V}}_{i}$, resulta

$$
\begin{aligned}
\int_{\widetilde{\mathrm{V}}_{i}}\left[w\left(\mathrm{x}_{i}\right)-w(\mathrm{x})\right] d s(\mathrm{x}) & =\int_{\widetilde{\mathrm{V}}_{i}} \nabla w^{\Omega}(\mathrm{x}) \cdot\left(\mathrm{x}-\mathrm{x}_{i}\right) d s(\mathrm{x}) \\
& +\int_{\widetilde{\mathrm{V}}_{i}} \int_{0}^{1} H\left(w^{\Omega}\right)\left(\mathrm{x}_{i}+t\left(\mathrm{x}-\mathrm{x}_{i}\right)\right)\left(\mathrm{x}-\mathrm{x}_{i}\right) \cdot\left(\mathrm{x}-\mathrm{x}_{i}\right) t d t d s(\mathrm{x}),
\end{aligned}
$$

onde $H$ denota a matriz Hessiana de $w^{\Omega}$. Consideremos $\mathrm{x}_{i}^{*}$ o centroide esférico de $\widetilde{\mathrm{V}}_{i}$ não restrito a $\mathbb{S}^{2}, \log \mathrm{O}$

$$
\begin{aligned}
\int_{\widetilde{\mathrm{V}}_{i}}\left[w\left(\mathrm{x}_{i}\right)-w(\mathrm{x})\right] d s(\mathrm{x})= & \int_{\widetilde{\mathrm{V}}_{i}} \nabla w^{\Omega}(\mathrm{x}) \cdot\left(\mathrm{x}-\mathrm{x}_{i}^{*}\right) d s(\mathrm{x})+\int_{\widetilde{\mathrm{V}}_{i}} \nabla w^{\Omega}(\mathrm{x}) \cdot\left(\mathrm{x}_{i}^{*}-\mathrm{x}_{i}\right) d s(\mathrm{x}) \\
& +\int_{\widetilde{\mathrm{V}}_{i}} \int_{0}^{1} H\left(w^{\Omega}\right)\left(\mathrm{x}_{i}+t\left(\mathrm{x}-\mathrm{x}_{i}\right)\right)\left(\mathrm{x}-\mathrm{x}_{i}\right) \cdot\left(\mathrm{x}-\mathrm{x}_{i}\right) t d t d s(\mathrm{x}) \\
= & I_{1}+I_{2}+I_{3} .
\end{aligned}
$$

Para $I_{1}$, usamos o critério da SCVT, a condição $\int_{\widetilde{\mathrm{V}}_{i}}\left(\mathrm{x}-\mathrm{x}_{i}^{*}\right) d s(\mathrm{x})=0$. Seja $\Pi_{\widetilde{\mathcal{V}}}$ a $L^{2}$-projeção no espaço $\widetilde{V}_{h}$ (constante por pedaços) e lembrando que $\nabla_{s} w(\mathrm{x})=\nabla w^{\Omega}(\mathrm{x})$ para cada $\mathrm{x} \in \widetilde{\mathrm{V}}_{i}$ temos da desigualdade de Cauchy-Schwarz que

$$
\begin{aligned}
I_{1} & =\int_{\widetilde{\mathrm{V}}_{i}} \nabla_{s} w(\mathrm{x}) \cdot\left(\mathrm{x}-\mathrm{x}_{i}^{*}\right) d s(\mathrm{x})-\Pi_{\widetilde{\mathcal{V}}}\left(\nabla_{s} w\right) \cdot \int_{\widetilde{\mathrm{V}}_{i}}\left(\mathrm{x}-\mathrm{x}_{i}^{*}\right) d s(\mathrm{x}) \\
& =\int_{\widetilde{\mathrm{V}}_{i}}\left[\nabla_{s} w(\mathrm{x})-\Pi_{\widetilde{\mathcal{V}}}\left(\nabla_{s} w\right)\right] \cdot\left(\mathrm{x}-\mathrm{x}_{i}^{*}\right) d s(\mathrm{x}) \\
& \leq\left(\int_{\widetilde{\mathrm{V}}_{i}}\left|\nabla_{s} w(\mathrm{x})-\Pi_{\widetilde{\mathcal{V}}}\left(\nabla_{s} w\right)\right|^{2} d s(\mathrm{x})\right)^{1 / 2}\left(\int_{\widetilde{\mathrm{V}}_{i}}\left\|\mathrm{x}-\mathrm{x}_{i}^{*}\right\|^{2} d s(\mathrm{x})\right)^{1 / 2} \\
& \leq C h S\left(\widetilde{\mathrm{V}}_{i}\right)^{1 / 2}\left\|\nabla_{s} w-\Pi_{\widetilde{\mathcal{V}}}\left(\nabla_{s} w\right)\right\|_{L^{2}\left(\widetilde{\mathrm{V}}_{i}\right)} \\
& \leq C h^{2} S\left(\widetilde{\mathrm{V}}_{i}\right)^{1 / 2}\|w\|_{H^{2}\left(\widetilde{\mathrm{V}}_{i}\right)} .
\end{aligned}
$$

Para $I_{2}$, como $\mathrm{x}_{i}=\mathcal{P}\left(\mathrm{x}_{i}^{*}\right)$ é o centroide esférico, da Proposição 3.8 segue que $\left\|\mathrm{x}_{i}^{*}-\mathrm{x}_{i}\right\| \leq C h^{2}$. Em virtude da desigualdade de Cauchy-Schwarz, tem-se

$$
\begin{aligned}
I_{2}=\int_{\widetilde{\mathrm{V}}_{i}} \nabla_{s} w(\mathrm{x}) \cdot\left(\mathrm{x}_{i}^{*}-\mathrm{x}_{i}\right) d s(\mathrm{x}) & \leq C h^{2} S\left(\widetilde{\mathrm{V}}_{i}\right)^{1 / 2}\left(\int_{\widetilde{\mathrm{V}}_{i}}\left|\nabla_{s} w(\mathrm{x})\right|^{2} d s(\mathrm{x})\right)^{1 / 2} \\
& \leq C h^{2} S\left(\widetilde{\mathrm{V}}_{i}\right)^{1 / 2}\|w\|_{H^{2}\left(\widetilde{\mathrm{V}}_{i}\right)} .
\end{aligned}
$$

Por último $I_{3}$, tomando o conjunto $\widetilde{\mathrm{V}}_{i}^{t}=\left\{\mathrm{x}^{*} \in \Omega_{h}: \mathrm{x}^{*}=\mathrm{x}_{i}+t\left(\mathrm{x}-\mathrm{x}_{i}\right) \quad \operatorname{com} \mathrm{x} \in \widetilde{\mathrm{V}}_{i}\right\}$. Pela mudança de variável, assumindo que $d s(\mathrm{x}) \leq 2 d s\left(\mathrm{x}^{*}\right) / t^{2}$ e dado que $S\left(\widetilde{\mathrm{V}}_{i}^{t}\right) \leq t^{2} S\left(\widetilde{\mathrm{V}}_{i}\right)$, temos 
pela desigualdade de Cauchy-Schwarz,

$$
\begin{aligned}
I_{3} & =\int_{\widetilde{\mathrm{V}}_{i}} \int_{0}^{1} H\left(w^{\Omega}\right)\left(\mathrm{x}_{i}+t\left(\mathrm{x}-\mathrm{x}_{i}\right)\right)\left(\mathrm{x}-\mathrm{x}_{i}\right) \cdot\left(\mathrm{x}-\mathrm{x}_{i}\right) t d t d s(\mathrm{x}) \\
& \leq 2 h^{2} \int_{0}^{1} \int_{\widetilde{\mathrm{V}}_{i}^{t}} \frac{\left|H\left(w^{\Omega}\right)\left(\mathrm{x}^{*}\right)\right|}{t} d s\left(\mathrm{x}^{*}\right) d t \\
& \leq 2 h^{2} \int_{0}^{1}\left(\int_{\widetilde{\mathrm{V}}_{i}^{t}}\left|H\left(w^{\Omega}\right)\left(\mathrm{x}^{*}\right)\right|^{2} d s\left(\mathrm{x}^{*}\right)\right)^{1 / 2} S\left(\widetilde{\mathrm{V}}_{i}\right)^{1 / 2} d t \\
& \leq C h^{2} S\left(\widetilde{\mathrm{V}}_{i}\right)^{1 / 2}\|w\|_{H^{2}\left(\widetilde{\mathrm{V}}_{i}\right)} .
\end{aligned}
$$

Combinando (4.15)-(4.17) e usando um argumento de densidade para $w \in H^{2}\left(\mathbb{S}^{2}\right)$, obteremos o resultado.

O seguinte lema é o passo essencial na obtenção da ordem quadrática e usa o fato anterior.

Lema 4.3. Sejam $f \in H_{Q}^{1}\left(\mathbb{S}^{2}\right)$ e $\widetilde{\mathcal{V}}=\left\{\mathrm{x}_{i}, \widetilde{\mathrm{V}}_{i}\right\}_{i=1}^{N}$ uma decomposição dual de Voronö̈ do tipo SCVT de $\mathbb{S}^{2}$ que satisfaz as condiçôes $(2.5 \mathrm{a})-(2.5 \mathrm{c})$. Então, para cada $w \in H^{2}\left(\mathbb{S}^{2}\right) \cap H_{Q}^{1}\left(\mathbb{S}^{2}\right)$, existe uma constante $C \in \mathbb{R}_{+}$, independente de $h$, tal que

$$
\left(f, \Pi_{h}(w)-\widetilde{\Pi}_{h}(w)\right) \leq C h^{2}\|f\|_{H^{1}\left(\mathbb{S}^{2}\right)}\|w\|_{H^{2}\left(\mathbb{S}^{2}\right)},
$$

onde $\Pi_{h}$ e $\widetilde{\Pi}_{h}$ são os interpoladores nos espaços $U_{h}$ e $\widetilde{V}_{h}$ respectivamente.

Demonstração. Pela definição do produto escalar,

$$
\begin{aligned}
\left(f, \Pi_{h}(w)-\widetilde{\Pi}_{h}(w)\right)= & \int_{\mathbb{S}^{2}} f(\mathrm{x})\left[\Pi_{h}(w)(\mathrm{x})-\widetilde{\Pi}_{h}(w)(\mathrm{x})\right] d s(\mathrm{x}) \\
= & \int_{\mathbb{S}^{2}} f(\mathrm{x})\left[\Pi_{h}(w)(\mathrm{x})-w(\mathrm{x})\right] d s(\mathrm{x})+\int_{\mathbb{S}^{2}} f(\mathrm{x})\left[w(\mathrm{x})-\widetilde{\Pi}_{h}(w)(\mathrm{x})\right] d s(\mathrm{x}) \\
= & \sum_{\widetilde{\mathrm{T}}_{i j k} \in \widetilde{\mathcal{T}}_{h}} \int_{\widetilde{T}_{i j k}} f(\mathrm{x})\left[\Pi_{h}(w)(\mathrm{x})-w(\mathrm{x})\right] d s(\mathrm{x}) \\
& +\sum_{i=1}^{N} \int_{\widetilde{\mathrm{V}}_{i}} f(\mathrm{x})\left[w(\mathrm{x})-\widetilde{\Pi}_{h}(w)(\mathrm{x})\right] d s(\mathrm{x}) .
\end{aligned}
$$

Somando e subtraindo $\Pi_{\widetilde{\mathcal{V}}}(f)$, a $L^{2}$-projeção da função $f$, segue

$$
\begin{aligned}
\left(f, \Pi_{h}(w)-\widetilde{\Pi}_{h}(w)\right)= & \sum_{\widetilde{\mathrm{T}}_{i j k} \in \widetilde{\mathcal{T}}_{h}} \int_{\widetilde{\mathrm{T}}_{i j k}} f(\mathrm{x})\left[\Pi_{h}(w)(\mathrm{x})-w(\mathrm{x})\right] d s(\mathrm{x}) \\
& +\sum_{i=1}^{N} \int_{\widetilde{\mathrm{V}}_{i}}\left[f(\mathrm{x})-\Pi_{\widetilde{\mathcal{V}}}(f)\right]\left[w(\mathrm{x})-\widetilde{\Pi}_{h}(w)(\mathrm{x})\right] d s(\mathrm{x}) \\
& +\sum_{i=1}^{N} \int_{\widetilde{\mathrm{V}}_{i}} \Pi_{\widetilde{\mathcal{V}}}(f)\left[w(\mathrm{x})-\widetilde{\Pi}_{h}(w)(\mathrm{x})\right] d s(\mathrm{x}) \\
= & I_{1}+I_{2}+I_{3}
\end{aligned}
$$


Usando a desigualdade de Cauchy-Schwarz e a Proposição 3.10 obtemos para $I_{1}$,

$$
\begin{aligned}
I_{1}=\sum_{\widetilde{\mathrm{T}}_{i j k} \in \widetilde{\mathcal{T}}_{h}} \int_{\widetilde{\mathrm{T}}_{i j k}} f(\mathrm{x})\left[\Pi_{h}(w)(\mathrm{x})-w(\mathrm{x})\right] d s(\mathrm{x}) \leq & \sum_{\widetilde{\mathrm{T}}_{i j k} \in \widetilde{\mathcal{T}}_{h}}\left(\int_{\widetilde{\mathrm{T}}_{i j k}}|f(\mathrm{x})|^{2} d s(\mathrm{x})\right)^{1 / 2} \\
& \times\left(\int_{\widetilde{\mathrm{T}}_{i j k}}\left|\Pi_{h}(w)(\mathrm{x})-w(\mathrm{x})\right|^{2} d s(\mathrm{x})\right)^{1 / 2} \\
& \leq \sum_{\widetilde{\mathrm{T}}_{i j k} \in \widetilde{\mathcal{T}}_{h}}\|f\|_{L^{2}\left(\widetilde{\mathrm{T}}_{i j k}\right)}\left\|w-\Pi_{h}(w)\right\|_{L^{2}\left(\widetilde{\mathrm{T}}_{i j k}\right)} \\
\leq & C h^{2}\|f\|_{L^{2}\left(\mathbb{S}^{2}\right)}\|w\|_{H^{2}\left(\mathbb{S}^{2}\right)} .
\end{aligned}
$$

De forma análoga, usando a Proposição 4.1 obtemos para $I_{2}$,

$$
\begin{aligned}
I_{2}=\sum_{i=1}^{N} \int_{\widetilde{\mathrm{V}}_{i}}\left[f(\mathrm{x})-\Pi_{\widetilde{\mathcal{V}}}(f)\right]\left[w(\mathrm{x})-\widetilde{\Pi}_{h}(w)(\mathrm{x})\right] d s(\mathrm{x}) \leq & \sum_{i=1}^{N}\left(\int_{\widetilde{\mathrm{V}}_{i}}\left|f(\mathrm{x})-\Pi_{\widetilde{\mathcal{V}}}(f)(\mathrm{x})\right|^{2} d s(\mathrm{x})\right)^{1 / 2} \\
& \times\left(\int_{\widetilde{\mathrm{V}}_{i}}\left|w(\mathrm{x})-\widetilde{\Pi}_{h}(w)(\mathrm{x})\right|^{2} d s(\mathrm{x})\right)^{1 / 2} \\
& \leq C h \sum_{i=1}^{N}\|f\|_{H^{1}\left(\widetilde{\mathrm{V}}_{i}\right)}\left\|w-\widetilde{\Pi}_{h}(w)\right\|_{L^{2}\left(\widetilde{\mathrm{V}}_{i}\right)} \\
& \leq C h^{2}\|f\|_{H^{1}\left(\mathbb{S}^{2}\right)}\|w\|_{H^{2}\left(\mathbb{S}^{2}\right)} .
\end{aligned}
$$

Por último, usando o Lema 4.2, temos para $I_{3}$

$$
\begin{aligned}
& I_{3}=\sum_{i=1}^{N} \int_{\widetilde{\mathrm{V}}_{i}} \Pi_{\widetilde{\mathcal{V}}}(f)\left[w(\mathrm{x})-\widetilde{\Pi}_{h}(w)(\mathrm{x})\right] d s(\mathrm{x}) \leq C h^{2} \sum_{i=1}^{N}\left|\Pi_{\widetilde{\mathcal{V}}}(f)\right|_{\widetilde{\mathrm{V}}_{i}} S\left(\widetilde{\mathrm{V}}_{i}\right)^{1 / 2}\|w\|_{H^{2}\left(\widetilde{\mathrm{V}}_{i}\right)} \\
& \leq C h^{2}\left(\left.\sum_{i=1}^{N} S\left(\widetilde{\mathrm{V}}_{i}\right)\left|\Pi_{\widetilde{\mathcal{V}}}(f)\right|_{\widetilde{\mathrm{V}}_{i}}\right|^{2}\right)^{1 / 2}\left(\sum_{i=1}^{N}\|w\|_{H^{2}\left(\widetilde{\mathrm{V}}_{i}\right)}\right)^{1 / 2} \\
& \leq C h^{2}\|f\|_{L^{2}\left(\mathbb{S}^{2}\right)}\|w\|_{H^{2}\left(\mathbb{S}^{2}\right)} \text {. }
\end{aligned}
$$

Combinando (4.18)-(4.20) obtemos o desejado.

Mostramos então a modificação sutil da prova de Du e Ju (2005), assumindo que a solução exata está no espaço $H^{2}\left(\mathbb{S}^{2}\right) \cap H_{Q}^{1}\left(\mathbb{S}^{2}\right)$ e o termo fonte em $H_{Q}^{1}\left(\mathbb{S}^{2}\right)$.

Teorema 4.3. Seja $\widetilde{\mathcal{V}}=\left\{\mathrm{x}_{i}, \widetilde{\mathrm{V}}_{i}\right\}_{i=1}^{N}$ uma decomposição dual de Voronö̈ do tipo SCVT de $\mathbb{S}^{2}$ que satisfaz as condiçôes $(2.5 \mathrm{~b})-(2.5 \mathrm{c})$ com função de densidade constante $\rho(\mathrm{x})>0$, para cada $\mathrm{x} \in \mathbb{S}^{2}$. Assuma também que $f \in H_{Q}^{1}\left(\mathbb{S}^{2}\right)$ e que $u \in H^{2}\left(\mathbb{S}^{2}\right) \cap H_{Q}^{1}\left(\mathbb{S}^{2}\right)$ é a única solução fraca de (1.8). Seja $\mathrm{F}_{i j}^{d}$ o fluxo discreto definido em (3.8), tal que o problema discreto (3.23) possui única solução $u_{h} \in \widetilde{U}_{h}$. Então, existe uma constante $C \in \mathbb{R}_{+}$, independente de $h$, tal que

$$
\left\|\varepsilon_{h}\right\|_{L^{2}\left(\mathbb{S}^{2}\right)} \leq C h^{2}\left(\|u\|_{H^{2}\left(\mathbb{S}^{2}\right)}+\|f\|_{H^{1}\left(\mathbb{S}^{2}\right)}\right)
$$

onde $\varepsilon_{h}=u-u_{h}$.

Demonstração. A demonstração é análoga ao Teorema 4.2 para a NOPT, modificando apenas a equação (4.11), usando o Lema 4.3, para garantir a potencia extra no termo $I_{2}$, i.e.,

$$
I_{2}=\left|\left(f, w_{h}-\mathrm{I}_{h}\left(w_{h}\right)\right)\right| \leq C h^{2}\|f\|_{L^{2}\left(\mathbb{S}^{2}\right)}\|w\|_{H^{2}\left(\mathbb{S}^{2}\right)} \leq C h^{2}\|f\|_{L^{2}\left(\mathbb{S}^{2}\right)}\left\|u-u_{h}\right\|_{L^{2}\left(\mathbb{S}^{2}\right)},
$$


onde $w_{h}=\Pi_{h}(w)$. Em consequência, é mostrada a ordem quadrática das soluções quando a malha é de tipo SCVT.

\subsection{Estimativas na max-norma}

Vamos passar às estimativas de erro nas max e $W^{1, \infty}$-normas para o nosso MVF. Vamos proceder usando a forma variacional (1.5) para as funções de Green regularizadas, as quais serão consideradas como funções auxiliares. Começamos introduzindo de Aubin (2013) as propriedades dessas funções de Green para $\mathbb{S}^{2}$ aliadas da maquinaria construída para as estimativas pontuais dos MEF em superfícies definida principalmente por Demlow (2009) e Kröner (2017).

\subsubsection{Funções de Green em $\mathbb{S}^{2}$}

O Lema abaixo contém propriedades fundamentais das funções de Green regularizadas para $\mathbb{S}^{2}$. A prova detalhada está contida no texto de Aubin (2013, Teorema, 4.13, pp. 108).

Lema 4.4 (Aubin, 2013). Existe G(x,y), uma função de Green do Laplaciano que satisfaz as seguintes propriedades:

1. Para cada $u \in C^{2}\left(\mathbb{S}^{2}\right)$,

$$
u(\mathrm{y})-\frac{1}{S\left(\mathbb{S}^{2}\right)} \int_{\mathbb{S}^{2}} u(\mathrm{x}) d s(\mathrm{x})=-\int_{\mathbb{S}^{2}} G(\mathrm{x}, \mathrm{y}) \Delta_{s} u(\mathrm{x}) d s(\mathrm{x})=\int_{\mathbb{S}^{2}} \nabla_{s} G(\mathrm{x}, \mathrm{y}) \cdot \nabla_{s} u(\mathrm{x}) d s(\mathrm{x}),
$$

onde $\nabla_{s}$ e $\Delta_{s}$ denotam o gradiente tangencial e o Laplaciano que atuam em funções de $\mathrm{x}$.

2. Para cada $\mathrm{x}, \mathrm{y} \in \mathbb{S}^{2}$ com $\mathrm{x} \neq \mathrm{y}$, segue $G(\mathrm{x}, \mathrm{y})$ é uma função $C^{\infty}\left(\mathbb{S}^{2} \times \mathbb{S}^{2} \backslash\{\mathrm{x}=\mathrm{y}\}\right)$.

3. Existe uma constante $C \in \mathbb{R}_{+}$tal que para cada $\mathrm{x}, \mathrm{y} \in \mathbb{S}^{2}$ com $\mathrm{x} \neq \mathrm{y}$,

$$
\left.|G(\mathrm{x}, \mathrm{y})| \leq C(1+\ln |\mathrm{d}(\mathrm{x}, \mathrm{y})|), \quad\left|\nabla_{s} G(\mathrm{x}, \mathrm{y})\right| \leq C \frac{1}{|\mathrm{~d}(\mathrm{x}, \mathrm{y})|}, \quad \mid \Delta_{s} G(\mathrm{x}, \mathrm{y})\right) \mid \leq C \frac{1}{|\mathrm{~d}(\mathrm{x}, \mathrm{y})|^{2}} .
$$

4. Por último, $G(\mathrm{x}, \mathrm{y})$ satisfaz

$$
\int_{\mathbb{S}^{2}} G(\mathrm{x}, \mathrm{y}) d s(\mathrm{x})=0
$$

Formalmente, se para um ponto fixado y $\in \mathbb{S}^{2}, G(\mathrm{x}, \mathrm{y})$ cumpre as condições do lema anterior, então é solução ao problema

$$
-\Delta_{s} G(\mathrm{x}, \mathrm{y})=\delta(\mathrm{x}-\mathrm{y})-\frac{1}{S\left(\mathbb{S}^{2}\right)}, \quad \mathrm{x} \in \mathbb{S}^{2},
$$

onde $\delta(\mathrm{x}-\mathrm{y})$ representa a função delta de Dirac discreta (Dirac delta function) no ponto x. Para simplificar a notação, vamos denotar a função $G(\mathrm{x}, \mathrm{y})$ por $G^{\mathrm{y}}(\mathrm{x})$ e $\delta(\mathrm{x}-\mathrm{y})$ por $\delta^{\mathrm{y}}(\mathrm{x})$ com y $\in \mathbb{S}^{2}$ fixo. Nessa ordem de ideias, a única solução $u \in H^{2}\left(\mathbb{S}^{2}\right) \cap H_{Q}^{1}\left(\mathbb{S}^{2}\right)$ de (1.4) pode ser representada por

$$
u(\mathrm{y})=\int_{\mathbb{S}^{2}} G^{\mathrm{y}}(\mathrm{x}) f(\mathrm{x}) d s(\mathrm{x}) .
$$

Desta maneira,

$$
-\Delta_{s} u(\mathrm{y})=-\int_{\mathbb{S}^{2}} \Delta_{s} G^{\mathrm{y}}(\mathrm{x}) f(\mathrm{x}) d s(\mathrm{x})=\int_{\mathbb{S}^{2}}\left[\delta^{\mathrm{y}}(\mathrm{x})-\frac{1}{\mathbb{S}^{2}}\right] f(\mathrm{x}) d s(\mathrm{x})=f(\mathrm{y}),
$$

dado que $f$ satisfaz a condição de compatibilidade (1.3), obtemos (1.4). Vamos usar a notação $\hat{G}^{\mathrm{y}}$ para nos referirnos à função de Green discreta definida como

$$
\hat{G}^{\mathrm{y}}(\mathrm{z}):=\int_{\mathbb{S}^{2}} G^{\mathrm{z}}(\mathrm{x}) \delta^{\mathrm{y}}(\mathrm{x}) d s(\mathrm{x}), \quad \text { para cada } \mathrm{z} \in \mathbb{S}^{2} .
$$


Além disso, empregaremos algumas características da função $\delta^{y}$, as quais são definidas na seguinte proposição, cujos detalhes da prova para superfícies em geral estão no trabalho de Demlow (2009, Proposição 4.2, pp. 813).

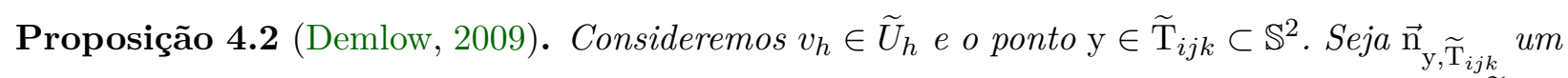
vetor unitário contido no plano tangente $\mathbb{T}_{\mathbb{S}^{2}, \mathrm{y}}$ no ponto y. Então, existem funções $\delta^{\mathrm{y}} \in C_{0}^{\infty}\left(\widetilde{\mathrm{T}}_{i j k}\right)$ e $\delta^{\mathrm{y}, \Omega} \in C_{0}^{\infty}\left(\Omega_{i j k}\right)$, a sua expansão ao conjunto $\Omega_{i j k}=\left\{\mathrm{x}^{*} \in \Omega_{h}: \mathcal{P}\left(\mathrm{x}^{*}\right) \in \widetilde{\mathrm{T}}_{i j k}\right\}$, que independem de $v_{h}$, tal que

$$
\left\|\delta^{\mathrm{y}}\right\|_{W^{m, p}\left(\widetilde{\mathrm{T}}_{i j k}\right)}+\left\|\delta^{\mathrm{y}, \Omega}\right\|_{W^{m, p}\left(\widetilde{\mathrm{T}}_{i j k}\right)} \leq C h^{-m-2\left(\frac{p-1}{p}\right)},
$$

para $m=0,1$ e $1 \leq p \leq \infty$. Além disso, existem constantes genéricas $C \in \mathbb{R}_{+}$, tal que

$$
\begin{aligned}
& \left|v_{h}(\mathrm{y})\right| \leq C\left|\int_{\widetilde{T}_{i j k}} \delta^{\mathrm{y}}(\mathrm{x}) v_{h}(\mathrm{x}) d s(\mathrm{x})\right| \\
& \left|\nabla_{s} v_{h}(\mathrm{y}) \cdot \overrightarrow{\mathrm{n}}_{\mathrm{y}, \widetilde{\mathrm{T}}_{i j k}}\right| \leq C\left|\int_{\widetilde{\mathrm{T}}_{i j k}} v_{h}(\mathrm{x}) \nabla_{s} \cdot \delta^{\mathrm{y}, \Omega}(\mathrm{x}) d s(\mathrm{x})\right| .
\end{aligned}
$$

Demonstração. Seja y $=\mathcal{P}\left(\mathrm{y}^{*}\right)$ e $\widetilde{\mathrm{T}}_{i j k}=\mathcal{P}\left(\mathrm{T}_{i j k}\right)$, para cada $\mathrm{y}^{*} \in \mathrm{T}_{i j k}$. Então, para qualquer $v_{h} \in \widetilde{U}_{h}$, temos $v_{h}(\mathrm{y})=\bar{v}_{h}^{\Omega}\left(\mathrm{y}^{*}\right)$. De acordo com Schatz e Wahlbin (1995) e Rannacher e Scott (1982), existe uma função suave $\delta^{\mathrm{y}^{*}}$ suportada em $\mathrm{T}_{i j k} \in \mathcal{T}_{h}$ e independente de $v_{h}$, que satisfaz (4.24), logo

$$
\bar{v}_{h}^{\Omega}\left(\mathrm{y}^{*}\right)=\int_{\mathrm{T}_{i j k}} \bar{v}_{h}^{\Omega}\left(\mathrm{x}^{*}\right) \delta^{\mathrm{y}^{*}}\left(\mathrm{x}^{*}\right) d s\left(\mathrm{x}^{*}\right) \leq C \int_{\widetilde{T}_{i j k}} v_{h}(\mathrm{x}) \delta^{\mathrm{y}}(\mathrm{x}) d s(\mathrm{x}) .
$$

Portanto,

$$
\left|v_{h}(\mathrm{y})\right| \leq C\left|\int_{\widetilde{\mathrm{T}}_{i j k}} v_{h}(\mathrm{x}) \delta^{\mathrm{y}}(\mathrm{x}) d s(\mathrm{x})\right| .
$$

Agora, dado o vetor $\overrightarrow{\mathrm{n}}_{\mathrm{y}, \widetilde{\mathrm{T}}_{i j k}} \in \mathbb{T}_{\mathbb{S}^{2}, \mathrm{y}}$ e utilizando a Proposição 3.8 , resulta

$$
\left|\nabla_{s} v_{h}(\mathrm{y}) \cdot \overrightarrow{\mathrm{n}}_{\mathrm{y}, \widetilde{\mathrm{T}}_{i j k}}\right| \leq C\left|\nabla \bar{v}_{h}^{\Omega}\left(\mathrm{y}^{*}\right) \cdot \overrightarrow{\mathrm{n}}_{\mathrm{y}, \widetilde{\mathrm{T}}_{i j k} \mid}\right|
$$

De forma análoga, existe uma função suave $\delta^{\mathrm{y}^{*}}$ suportada no elemento $\mathrm{T}_{i j k} \in \mathcal{T}_{h}$ que satisfaz (4.24), $\log 0$

$$
\nabla \bar{v}_{h}^{\Omega}\left(\mathrm{y}^{*}\right) \cdot \overrightarrow{\mathrm{n}}_{\mathrm{y}, \widetilde{\mathrm{T}}_{i j k}}=\int_{\mathrm{T}_{i j k}} \nabla \bar{v}^{\Omega}\left(\mathrm{x}^{*}\right) \cdot \overrightarrow{\mathrm{n}}_{\mathrm{y}, \widetilde{\mathrm{T}}_{i j k}} \delta^{\mathrm{y}^{*}}\left(\mathrm{x}^{*}\right) d s\left(\mathrm{x}^{*}\right) .
$$

A integração por partes produz

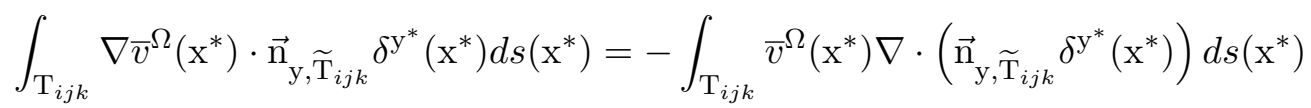

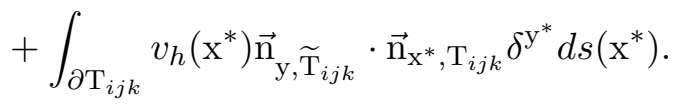

A última integral é zero em vista de $\delta^{\mathrm{y}^{*}}$ estar suportado em $\mathrm{T}_{i j k}$. Por último, considerando a mudança de variável, obtemos

$$
\left|\nabla_{s} v_{h}(\mathrm{y}) \cdot \overrightarrow{\mathrm{n}}_{\mathrm{y}, \widetilde{\mathrm{T}}_{i j k}}\right| \leq C\left|\int_{\widetilde{\mathrm{T}}_{i j k}} v_{h}(\mathrm{x}) \nabla_{s} \cdot \delta^{\mathrm{y}, \Omega} d s(\mathrm{x})\right|,
$$

onde $\delta^{\mathrm{y}, \Omega}=\left\|\mathrm{x}^{*}\right\| \delta^{\mathrm{y}^{*}, \Omega} \overrightarrow{\mathrm{n}}_{\mathrm{y}, \widetilde{\mathrm{T}}_{i j k}}$. Isto encerra a demonstração.

Vamos introduzir uma forma variacional para a função de Green discreta $\hat{G}^{\mathrm{y}}$. Consideremos 
$\mathrm{y} \in \mathbb{S}^{2}$ fixo, definimos o funcional bilinear,

$$
\mathcal{A}\left(v, \hat{G}^{\mathrm{y}}\right)=\int_{\mathbb{S}^{2}} \nabla_{s} v(\mathrm{x}) \cdot \nabla_{s} \hat{G}^{\mathrm{y}}(\mathrm{x}) d s(\mathrm{x}), \quad \text { para cada } v \in H_{Q}^{1}\left(\mathbb{S}^{2}\right)
$$

onde $\nabla_{s, \mathrm{y}}$ atua no ponto x. Observe que, da fórmula de Green junto com (4.23), resulta para $v \in H_{Q}^{1}\left(\mathbb{S}^{2}\right)$ que

$$
\begin{aligned}
\int_{\mathbb{S}^{2}} \nabla_{s} v(\mathrm{x}) \cdot \nabla_{s} \hat{G}^{\mathrm{y}}(\mathrm{x}) d s(\mathrm{x})=-\int_{\mathbb{S}^{2}} v(\mathrm{x}) \Delta_{s} \hat{G}^{\mathrm{y}}(\mathrm{x}) d s(\mathrm{x}) & =\int_{\mathbb{S}^{2}} v(\mathrm{x})\left[\delta^{\mathrm{y}}(\mathrm{x})-\frac{1}{S\left(\mathbb{S}^{2}\right)}\right] d s(\mathrm{x}) \\
& =\int_{\mathbb{S}^{2}} v(\mathrm{x}) \delta^{\mathrm{y}}(\mathrm{x}) d s(\mathrm{x}) .
\end{aligned}
$$

Dizemos então, que as funções discretas de Green $\hat{G}_{0}^{\mathrm{y}}, \hat{G}_{1}^{\mathrm{y}} \in C^{\infty}\left(\mathbb{S}^{2}\right) \cap H_{Q}^{1}\left(\mathbb{S}^{2}\right)$ são as únicas soluções aos problemas variacionais adjuntos respectivamente

$$
\begin{aligned}
& \mathcal{A}\left(v, \hat{G}_{0}^{\mathrm{y}}\right)=\left(v, \delta^{\mathrm{y}}\right), \quad \text { para cada } v \in H_{Q}^{1}\left(\mathbb{S}^{2}\right), \\
& \mathcal{A}\left(v, \hat{G}_{1}^{\mathrm{y}}\right)=\left(v, \nabla_{s} \cdot \delta^{\mathrm{y}, \Omega}\right), \quad \text { para cada } v \in H_{Q}^{1}\left(\mathbb{S}^{2}\right) .
\end{aligned}
$$

Adicionalmente, por conta da construção de $\hat{G}_{n}^{\mathrm{y}}$ podemos incorporar sua aproximação por elementos finitos, digamos $\hat{G}_{n, h}^{\mathrm{y}} \in \widetilde{U}_{h}, n \in\{0,1\}$, que seja a única solução ao problema

$$
\mathcal{A}\left(w_{h}, \hat{G}_{n}^{\mathrm{y}}-\hat{G}_{n, h}^{\mathrm{y}}\right)=0, \quad \text { para cada } w_{h} \in \widetilde{U}_{h} .
$$

Claramente, $\hat{G}_{n, h}^{\mathrm{y}}$ satisfaz a condição $(4.22)$ dada a estrutura do espaço $\widetilde{U}_{h}$.

Deve-se notar que $\hat{G}_{n}^{\mathrm{y}}, n \in\{0,1\}$ e a sua aproximação pelo MEFL satisfazem as estimativas de aproximação dos MEF nas $H^{1}$ e $L^{2}$-normas. Além isso, precisamos de outras estimativas a priori das funções de Green que foram bem estudadas na literatura por Demlow (2009); Kröner (2017); Rannacher e Scott (1982) e Schatz e Wahlbin (1995). Reunimos algumas delas no seguinte lema.

Lema 4.5 (Demlow, 2009). Sejam $\hat{G}_{n}^{\mathrm{y}}$ a função de Green discreta e $\hat{G}_{n, h}^{\mathrm{y}} \in \widetilde{U}_{h}(n \in\{0,1\})$ a sua aproximação por elementos finitos. Então, têm-se:

$$
\begin{aligned}
\left\|\nabla_{s}\left(\hat{G}_{1}^{\mathrm{y}}-\hat{G}_{1, h}^{\mathrm{y}}\right)\right\|_{L^{1}\left(\mathbb{S}^{2}\right)} & \leq C|\ln h|, \\
\left\|\hat{G}_{0}^{\mathrm{y}}-\hat{G}_{0, h}^{\mathrm{y}}\right\|_{W^{1,1}\left(\mathbb{S}^{2}\right)} & \leq C h|\ln h|, \\
\left\|\nabla_{s} \hat{G}_{1}^{\mathrm{y}}\right\|_{L^{1}\left(\mathbb{S}^{2}\right)}+\left\|\hat{G}_{0}^{\mathrm{y}}\right\|_{W^{2,1}\left(\mathbb{S}^{2}\right)} & \leq C|\ln h|, \\
\left\|\hat{G}_{n, h}^{\mathrm{y}}\right\|_{H^{1}\left(\mathbb{S}^{2}\right)} & \leq C|\ln h|^{1 / 2}, \quad \text { com } n \in\{0,1\}, \\
\left\|\hat{G}_{n, h}^{\mathrm{y}}\right\|_{W^{1,1}\left(\mathbb{S}^{2}\right)} & \leq C|\ln h|, \quad \text { com } n \in\{0,1\} .
\end{aligned}
$$

onde $C \in \mathbb{R}_{+}$são constantes genéricas que independem de $h$. O fator $|\ln h|^{1 / 2}$ possui ordem $\mathcal{O}\left(h^{-\eta}\right)$ para $\eta \in(0,1)$.

Demonstração. Omitiremos os detalhes de (4.27a)-(4.27c), mas estão nos trabalhos de Demlow (2009, Lema 3.3, pp. 819) e Kröner (2017, Lema 5.2, pp. 10), que por sua vez, são as versões estendidas para superfícies das definidas por Rannacher e Scott (1982); Scott (1976) e Schatz (1998).

Para (4.27d), seja $\hat{G}_{n, h}^{\mathrm{y}} \in \widetilde{U}_{h}$, a solução por elemento finito de $\hat{G}_{n}^{\mathrm{y}}(n \in\{0,1\})$. Então, da Proposição 4.2 , existe uma função suave $\delta^{\mathrm{y}}$ suportada em $\widetilde{\mathrm{T}}_{i j k} \in \widetilde{\mathcal{T}}_{h}$, tal que para $\mathrm{z} \in \mathbb{S}^{2}$ fixo, segue

$$
\hat{G}_{n, h}^{\mathrm{y}}(\mathrm{z})=\int_{\mathbb{S}^{2}} \hat{G}_{n, h}^{\mathrm{y}}(\mathrm{x}) \delta^{\mathrm{z}}(\mathrm{x}) d s(\mathrm{x})=\mathcal{A}\left(\hat{G}_{n, h}^{\mathrm{y}}, \hat{G}_{n, h}^{\mathrm{y}}\right)=\left\|\hat{G}_{n, h}^{\mathrm{y}}\right\|_{H^{1}\left(\mathbb{S}^{2}\right)}^{2} .
$$

Segue da desigualdade de Sobolev discreta (veja Brenner e Scott, 2007, Lema 4.9.2, pp. 124), que 
existe uma constante $C \in \mathbb{R}_{+}$tal que

$$
\left|\hat{G}_{n, h}^{\mathrm{y}}(\mathrm{z})\right| \leq C|\ln h|^{1 / 2}\left\|\hat{G}_{n, h}^{\mathrm{y}}\right\|_{H^{1}\left(\mathbb{S}^{2}\right)}, \quad \text { com } n \in\{0,1\} .
$$

Combinando (4.28)-(4.29) obtemos (4.27d). Por último, (4.27e) segue de (4.27b) e (4.27c) usando a desigualdade triangular.

Com o anterior podemos mostrar uma estimativa fraca da estabilidade das soluções na maxnorma. Mais precisamente:

Lema 4.6 (Estabilidade fraca). Assuma que $u_{h} \in \widetilde{U}_{h}$ seja a única solução do problema discreto (3.23). Então, existe uma constante $C \in \mathbb{R}_{+}$, que independe de $h$, tal que

$$
\left\|u_{h}\right\|_{\infty, h} \leq C|\ln h|^{1 / 2}\left\|u_{h}\right\|_{H^{1}\left(\mathbb{S}^{2}\right)} .
$$

Demonstração. Pela Proposição 4.2, existe uma função suave $\delta^{\mathrm{y}} \in C_{0}^{\infty}\left(\widetilde{\mathrm{T}}_{i j k}\right)$, tal que

$$
\left|u_{h}(\mathrm{y})\right| \leq C\left|\int_{\mathbb{S}^{2}} u_{h}(\mathrm{x}) \delta^{\mathrm{y}}(\mathrm{x}) d s(\mathrm{x})\right|=C\left|\mathcal{A}\left(u_{h}, \hat{G}_{h}^{\mathrm{y}}\right)\right| .
$$

Em virtude da continuidade do funcional bilinear $\mathcal{A}(\cdot, \cdot)$ e o Lema 4.5 , resulta

$$
\left|u_{h}(\mathrm{y})\right| \leq C\left|\mathcal{A}\left(u_{h}, \hat{G}_{h}^{\mathrm{y}}\right)\right| \leq C\left\|u_{h}\right\|_{H^{1}\left(\mathbb{S}^{2}\right)}\left\|\hat{G}_{h}^{\mathrm{y}}\right\|_{H^{1}\left(\mathbb{S}^{2}\right)} \leq C|\ln h|^{1 / 2}\left\|u_{h}\right\|_{H^{1}\left(\mathbb{S}^{2}\right)} .
$$

Portanto, tomando o máximo, segue

$$
\left\|u_{h}\right\|_{\infty, h} \leq C|\ln h|^{1 / 2}\left\|u_{h}\right\|_{H^{1}\left(\mathbb{S}^{2}\right)}
$$

como se queria mostrar.

Agora, podemos enunciar uma primeira estimativa de erro das soluções dadas pelo esquema de volumes finitos na max-norma.

Teorema 4.4. Sejam $\widetilde{\mathcal{V}}=\left\{\mathrm{x}_{i}, \widetilde{\mathrm{V}}_{i}\right\}_{i=1}^{N}$ a decomposição Voronö̈-Delaunay (NOPT) para $\mathbb{S}^{2}$ que satisfaz as condições (2.5a)-(2.5c) e $f \in L^{2}\left(\mathbb{S}^{2}\right)$ que satisfaz a condição de compatibilidade (1.3). Assuma que a única solução fraca u de (1.8) pertence ao espaço $W^{2, \infty}\left(\mathbb{S}^{2}\right) \cap H^{2}\left(\mathbb{S}^{2}\right) \cap H_{Q}^{1}\left(\mathbb{S}^{2}\right)$. Seja $\mathrm{F}_{i j}^{d}$ o fluxo discreto definido em (3.8), tal que o problema discreto (3.23) possui única solução $u_{h} \in \widetilde{U}_{h}$. Então, existe uma constante $C \in \mathbb{R}_{+}$, independente de $h$, tal que

$$
\left\|\varepsilon_{h}\right\|_{L^{\infty}\left(\mathbb{S}^{2}\right)} \leq C h|\ln h|^{1 / 2}\left(\|u\|_{H^{2}\left(\mathbb{S}^{2}\right)}+\|f\|_{L^{2}\left(\mathbb{S}^{2}\right)}\right),
$$

$\operatorname{com} \varepsilon_{h}=u-u_{h}$.

Demonstração. Para y $\in \mathbb{S}^{2}$ fixo, consideremos a função discreta de Green $\hat{G}_{0}^{\mathrm{y}}$ que satisfaz o problema variacional adjunto (4.25a) e a condição (4.22). Consideramos também a sua aproximação por elementos Finitos $\hat{G}_{0, h}^{\mathrm{y}} \in \widetilde{U}_{h}$ que satisfaz a o problema variacional (4.26). Então, da Proposição 4.2, existe uma função suave $\delta^{y}$ suportada em $\widetilde{\mathrm{T}}_{i j k} \in \widetilde{\mathcal{T}}_{h}$ e pela Proposição 3.10 , temos que

$$
\begin{aligned}
\left|\left(u-u_{h}\right)(\mathrm{y})\right| & \leq\left|\left(u-\Pi_{h}(u)\right)(\mathrm{y})\right|+C\left|\int_{\mathbb{S}^{2}}\left(\Pi_{h}(u)-u_{h}\right)(\mathrm{x}) \delta^{\mathrm{y}}(\mathrm{x}) d s(\mathrm{x})\right| \\
& \leq C\left(\left\|u-\Pi_{h}(u)\right\|_{L^{\infty}\left(\mathbb{S}^{2}\right)}+\left|\mathcal{A}\left(\Pi_{h}(u)-u_{h}, \hat{G}_{0}^{\mathrm{y}}\right)\right|\right) \\
& \leq C\left(\left\|u-\Pi_{h}(u)\right\|_{L^{\infty}\left(\mathbb{S}^{2}\right)}+\left|\mathcal{A}\left(\Pi_{h}(u)-u_{h}, \hat{G}_{0}^{\mathrm{y}}-\hat{G}_{0, h}^{\mathrm{y}}+\hat{G}_{0, h}^{\mathrm{y}}\right)\right|\right) \\
& =C\left(\left\|u-\Pi_{h}(u)\right\|_{L^{\infty}\left(\mathbb{S}^{2}\right)}+\left|\mathcal{A}\left(\Pi_{h}(u)-u_{h}, \hat{G}_{0}^{\mathrm{y}}-\hat{G}_{0, h}^{\mathrm{y}}\right)+\mathcal{A}\left(\Pi_{h}(u)-u_{h}, \hat{G}_{0, h}^{\mathrm{y}}\right)\right|\right) .
\end{aligned}
$$


Agora, para a limitação: a Proposição 3.10, $\operatorname{com} p=\infty$, produz

$$
\left\|u-\Pi_{h}(u)\right\|_{L^{\infty}\left(\mathbb{S}^{2}\right)} \leq C h^{2}\|u\|_{W^{2, \infty}\left(\mathbb{S}^{2}\right)} .
$$

Como $\Pi_{h}(u)-u_{h} \in \widetilde{U}_{h}$, da equação (4.26), temos que $\mathcal{A}\left(\Pi_{h}(u)-u_{h}, \hat{G}_{0}^{\mathrm{y}}-\hat{G}_{0, h}^{\mathrm{y}}\right)=0$. Pela continuidade do funcional bilinear $\mathcal{A}(\cdot, \cdot)$ e invocando a Proposição 3.10 e a estimativa do Teorema 4.1 obtemos

$$
\begin{aligned}
\left|\mathcal{A}\left(\Pi_{h}(u)-u_{h}, \hat{G}_{0, h}^{\mathrm{y}}\right)\right| & \leq C\left\|\Pi_{h}(u)-u_{h}\right\|_{H^{1}\left(\mathbb{S}^{2}\right)}\left\|\hat{G}_{0, h}^{\mathrm{y}}\right\|_{H^{1}\left(\mathbb{S}^{2}\right)} \\
& \leq C\left(\left\|u-\Pi_{h}(u)\right\|_{H^{1}\left(\mathbb{S}^{2}\right)}+\left\|u-u_{h}\right\|_{H^{1}\left(\mathbb{S}^{2}\right)}\right)\left\|\hat{G}_{0, h}^{\mathrm{y}}\right\|_{H^{1}\left(\mathbb{S}^{2}\right)} \\
& \leq C h\left(\|u\|_{H^{2}\left(\mathbb{S}^{2}\right)}+\|f\|_{L^{2}\left(\mathbb{S}^{2}\right)}\right)\left\|\hat{G}_{0, h}^{\mathrm{y}}\right\|_{H^{1}\left(\mathbb{S}^{2}\right)} .
\end{aligned}
$$

Por fim, usando (4.27d) do Lema 4.5, temos

$$
\left|\mathcal{A}\left(\Pi_{h}(u)-u_{h}, G_{h}^{\mathrm{y}}\right)\right| \leq C h|\ln h|^{1 / 2}\left(\|u\|_{H^{2}\left(\mathbb{S}^{2}\right)}+\|f\|_{L^{2}\left(\mathbb{S}^{2}\right)}\right) .
$$

Desta maneira, combinando (4.30) e (4.31) para $h>0$ suficientemente pequeno, obtemos

$$
\left|\left(u-u_{h}\right)(\mathrm{y})\right| \leq C h|\ln h|^{1 / 2}\left(\|u\|_{H^{2}\left(\mathbb{S}^{2}\right)}+\|f\|_{L^{2}\left(\mathbb{S}^{2}\right)}\right),
$$

tomando o máximo segue o resultado desejado.

Observação 4.2. A condição da aproximação do esquema (3.11) na estimação acima está inclusa na estimativa da $H^{1}$-norma.

A estimativa acima não é ótima em relação à regularidade requerida da solução exata, i.e., $u \in H^{2}\left(\mathbb{S}^{2}\right) \cap H_{Q}^{1}\left(\mathbb{S}^{2}\right)$. Essa regularidade excessiva pode ser removida prosseguindo da seguinte forma: coloque a restrição na única solução fraca $u$ de (1.8) pertence o espaço $W^{2, \infty}\left(\mathbb{S}^{2}\right) \cap H_{Q}^{1}\left(\mathbb{S}^{2}\right)$. Após, calcule uma estimativa na $W^{1, \infty}$-norma usando as Proposições 3.10 e 4.2 e o Lema 4.5. Em seguida, estime a max-norma das soluções.

O resultado abaixo estabelecera uma estimação pontual para a aproximação do gradiente tangencial das soluções na max-norma.

Teorema 4.5. Sejam $\widetilde{\mathcal{V}}=\left\{\mathrm{x}_{i}, \widetilde{\mathrm{V}}_{i}\right\}_{i=1}^{N}$ a decomposição Voronö̈-Delaunay (NOPT) para $\mathbb{S}^{2}$ que satisfaz as condições (2.5a)-(2.5c) e $f \in L^{\infty}\left(\mathbb{S}^{2}\right)$ que satisfaz a condição de compatibilidade (1.3). Assuma que a única solução fraca u de (1.8) pertence ao espaço $W^{2, \infty}\left(\mathbb{S}^{2}\right) \cap H_{Q}^{1}\left(\mathbb{S}^{2}\right)$. Seja $\mathrm{F}_{i j}^{d}$ o fluxo discreto definido em (3.8), tal que o problema discreto (3.23) possui única solução $u_{h} \in \widetilde{U}_{h}$. Então, existem constantes $C, h_{0} \in \mathbb{R}_{+}$que independem de $u$, tal que para $0<h \leq h_{0}<1$ tem-se,

$$
\left\|\nabla_{s} \varepsilon_{h}\right\|_{L^{\infty}\left(\mathbb{S}^{2}\right)} \leq C h|\ln h|\left(\|u\|_{W^{2, \infty}\left(\mathbb{S}^{2}\right)}+\|f\|_{L^{\infty}\left(\mathbb{S}^{2}\right)}\right)
$$

$\operatorname{com} \varepsilon_{h}=u-u_{h}$.

Demonstração. Procederemos via um argumento de dualidade. Sejam y $\in \mathbb{S}^{2}$ fixo e o vetor tangente

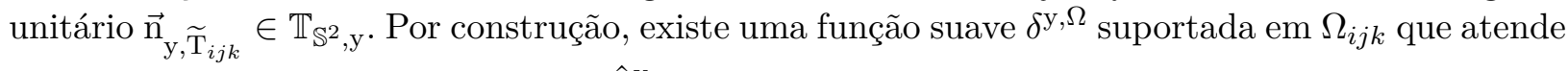
as condições da Proposição 4.2. Seja $\hat{G}_{1}^{\mathrm{y}}$ a função discreta de Green que junto a (4.22), satisfaz o problema variacional adjunto $(4.25 \mathrm{~b})$, i.e.,

$$
\mathcal{A}\left(v, \hat{G}_{1}^{\mathrm{y}}\right)=\left(v, \nabla_{s} \cdot \delta^{\mathrm{y}, \Omega}\right), \quad \text { para cada } v \in H_{Q}^{1}\left(\mathbb{S}^{2}\right) .
$$

Consideremos também $\hat{G}_{1, h}^{\mathrm{y}}$ a aproximação por Elementos Finitos de $\hat{G}_{1}^{\mathrm{y}}$, a única solução do problema (4.26) satisfazendo a condição (4.22). Então, da desigualdade triangular,

$$
\left|\nabla_{s}\left(u-u_{h}\right)(\mathrm{y}) \cdot \overrightarrow{\mathrm{n}}_{\mathrm{y}, \widetilde{\mathrm{T}}_{i j k}}\right| \leq\left|\nabla_{s}\left(u-\Pi_{h}(u)\right)(\mathrm{y}) \cdot \overrightarrow{\mathrm{n}}_{\mathrm{y}, \widetilde{\mathrm{T}}_{i j k}}\right|+\left|\nabla_{s}\left(\Pi_{h}(u)-u_{h}\right)(\mathrm{y}) \cdot \overrightarrow{\mathrm{n}}_{\mathrm{y}, \widetilde{\mathrm{T}}_{i j k}}\right| \cdot
$$


Da Proposição 3.10, o primeiro termo do lado direito é limitado por

$$
\left\|\nabla_{s}\left(u-\Pi_{h}(u)\right)\right\|_{L^{\infty}\left(\mathbb{S}^{2}\right)} \leq C h\|u\|_{W^{2, \infty}\left(\mathbb{S}^{2}\right)} .
$$

Basta limitar $\left|\nabla_{s}\left(\Pi_{h}(u)-u_{h}\right)(\mathrm{y}) \cdot \overrightarrow{\mathrm{n}}_{\mathrm{y}, \widetilde{\mathrm{T}}_{i j k}}\right| \cdot$ Com efeito, para $\Pi_{h}(u)-u_{h} \in \widetilde{U}_{h}$ teremos da linearidade de $\mathcal{A}(\cdot, \cdot)$ que

$$
\begin{aligned}
\mid \nabla_{s}\left(\Pi_{h}(u)-u_{h}\right)(\mathrm{y}) \cdot \overrightarrow{\mathrm{n}}_{\mathrm{y}, \widetilde{\mathrm{T}}_{i j k} \mid \leq} & C\left|\int_{\mathbb{S}^{2}}\left(\Pi_{h}(u)-u_{h}\right)(\mathrm{x}) \nabla_{s} \cdot \delta^{\mathrm{y}, \Omega}(\mathrm{x}) d s(\mathrm{x})\right| \\
\leq & C\left|\mathcal{A}\left(\Pi_{h}(u)-u_{h}, \hat{G}_{1}^{\mathrm{y}}\right)\right| \\
= & C\left(\left|\mathcal{A}\left(\Pi_{h}(u)-u_{h}, \hat{G}_{1}^{\mathrm{y}}-\hat{G}_{1, h}^{\mathrm{y}}+\hat{G}_{1, h}^{\mathrm{y}}\right)\right|\right) \\
\leq & C\left(\left|\mathcal{A}\left(\Pi_{h}(u)-u_{h}, \hat{G}_{1}^{\mathrm{y}}-\hat{G}_{1, h}^{\mathrm{y}}\right)\right|+\left|\mathcal{A}\left(\Pi_{h}(u)-u_{h}, \hat{G}_{1, h}^{\mathrm{y}}\right)\right|\right) \\
\leq & C\left(\left|\mathcal{A}\left(\Pi_{h}(u)-u_{h}, \hat{G}_{1}^{\mathrm{y}}-\hat{G}_{1, h}^{\mathrm{y}}\right)\right|+\left|\mathcal{A}\left(\Pi_{h}(u)-u, \hat{G}_{1, h}^{\mathrm{y}}\right)\right|\right. \\
& \left.+\left|\mathcal{A}\left(u-u_{h}, \hat{G}_{1, h}^{\mathrm{y}}\right)\right|\right) .
\end{aligned}
$$

Segue de (4.26) que $\mathcal{A}\left(\Pi_{h}(u)-u_{h}, \hat{G}_{1}^{\mathrm{y}}-\hat{G}_{1, h}^{\mathrm{y}}\right)=0$. Pela continuidade de $\mathcal{A}(\cdot, \cdot)$ junto à Proposição 3.10 e a estimativa (4.27e) do Lema 4.5, resulta

$$
\left|\mathcal{A}\left(\Pi_{h}(u)-u, \hat{G}_{1, h}^{\mathrm{y}}\right)\right| \leq C h|\ln h|\|u\|_{W^{2, \infty}\left(\mathbb{S}^{2}\right)} .
$$

Agora, para a limitação do termo $\left|\mathcal{A}\left(u-u_{h}, \hat{G}_{1, h}^{\mathrm{y}}\right)\right|$, usamos a linearidade de $\mathcal{A}(\cdot, \cdot)$, após somamos e subtraímos os funcionais $\mathcal{A}_{h}^{d}(\cdot, \cdot)$ e $\mathcal{A}_{h}(\cdot, \cdot)$, para obter

$$
\begin{aligned}
\left|\mathcal{A}\left(u-u_{h}, \hat{G}_{1, h}^{\mathrm{y}}\right)\right| \leq & \left|\mathcal{A}\left(u, \hat{G}_{1, h}^{\mathrm{y}}\right)-\mathcal{A}_{h}^{d}\left(u_{h}, \mathrm{I}_{h}\left(\hat{G}_{1, h}^{\mathrm{y}}\right)\right)\right|+\left|\mathcal{A}_{h}^{d}\left(u_{h}, \mathrm{I}_{h}\left(\hat{G}_{1, h}^{\mathrm{y}}\right)\right)-\mathcal{A}_{h}\left(u_{h}, \mathrm{I}_{h}\left(\hat{G}_{1, h}^{\mathrm{y}}\right)\right)\right| \\
& +\left|\mathcal{A}_{h}\left(u_{h}, \mathrm{I}_{h}\left(\hat{G}_{1, h}^{\mathrm{y}}\right)\right)-\mathcal{A}\left(u_{h}, \hat{G}_{1, h}^{\mathrm{y}}\right)\right| \\
= & I_{1}+I_{2}+I_{3} .
\end{aligned}
$$

Para $I_{1}$, com $f \in L^{\infty}\left(\mathbb{S}^{2}\right)$, aplicando os Lemas 4.1 e 4.5 para $\hat{G}_{1, h}^{\mathrm{y}} \in \widetilde{U}_{h}$, obtemos

$$
\begin{aligned}
I_{1}=\left|\mathcal{A}\left(u, \hat{G}_{1, h}^{\mathrm{y}}\right)-\mathcal{A}_{h}^{d}\left(u_{h}, \mathrm{I}_{h}\left(\hat{G}_{1, h}^{\mathrm{y}}\right)\right)\right|=\left|\left(f, \hat{G}_{1, h}^{\mathrm{y}}-\mathrm{I}_{h}\left(\hat{G}_{1, h}^{\mathrm{y}}\right)\right)\right| & \leq C h\|f\|_{L^{\infty}\left(\mathbb{S}^{2}\right)}\left\|\hat{G}_{1, h}^{\mathrm{y}}\right\|_{W^{1,1}\left(\mathbb{S}^{2}\right)} \\
& \leq C h|\ln h|\|f\|_{L^{\infty}\left(\mathbb{S}^{2}\right)} .
\end{aligned}
$$

Para $I_{2}$, invocando o Lema 3.4 e a equação (4.27c), seque que

$$
\begin{aligned}
I_{2}=\left|\mathcal{A}_{h}^{d}\left(u_{h}, \mathrm{I}_{h}\left(\hat{G}_{1, h}^{\mathrm{y}}\right)\right)-\mathcal{A}_{h}\left(u_{h}, \mathrm{I}_{h}\left(\hat{G}_{1, h}^{\mathrm{y}}\right)\right)\right| & \leq C h^{2}\left\|\nabla_{s} u_{h}\right\|_{L^{\infty}\left(\mathbb{S}^{2}\right)}\left\|\nabla_{s} \hat{G}_{1, h}^{\mathrm{y}}\right\|_{L^{1}\left(\mathbb{S}^{2}\right)} \\
& \leq C h^{2}|\ln h|\left\|\nabla_{s} u_{h}\right\|_{L^{\infty}\left(\mathbb{S}^{2}\right)} .
\end{aligned}
$$

Note que, da Proposição 3.10, temos

$$
\begin{aligned}
\left\|\nabla_{s} u_{h}\right\|_{L^{\infty}\left(\mathbb{S}^{2}\right)} & \leq\left\|\nabla_{s}\left(\Pi_{h}(u)-u_{h}\right)\right\|_{L^{\infty}\left(\mathbb{S}^{2}\right)}+\left\|\nabla_{s}\left(\Pi_{h}(u)\right)\right\|_{L^{\infty}\left(\mathbb{S}^{2}\right)} \\
& \leq\left\|\nabla_{s}\left(\Pi_{h}(u)-u_{h}\right)\right\|_{L^{\infty}\left(\mathbb{S}^{2}\right)}+C h\|u\|_{W^{2, \infty}\left(\mathbb{S}^{2}\right)}+C\|u\|_{W^{2, \infty}\left(\mathbb{S}^{2}\right)} .
\end{aligned}
$$

Logo, tem-se

$$
I_{2} \leq C h^{2}|\ln h|\left(\left\|\nabla_{s}\left(\Pi_{h}(u)-u_{h}\right)\right\|_{L^{\infty}\left(\mathbb{S}^{2}\right)}+(h+1)\|u\|_{W^{2, \infty}\left(\mathbb{S}^{2}\right)}\right) .
$$


Para $I_{3}$, do Lema 3.5, e (4.27c) obtemos, de forma similar a $I_{2}$ que,

$$
\begin{aligned}
I_{3}=\left|\mathcal{A}_{h}\left(u_{h}, \mathrm{I}_{h}\left(\hat{G}_{1, h}^{\mathrm{y}}\right)\right)-\mathcal{A}\left(u_{h}, \hat{G}_{1, h}^{\mathrm{y}}\right)\right| & \leq C h^{2}\left\|\nabla_{s} u_{h}\right\|_{L^{\infty}\left(\mathbb{S}^{2}\right)}\left\|\nabla_{s} \hat{G}_{1, h}^{\mathrm{y}}\right\|_{L^{1}\left(\mathbb{S}^{2}\right)} \\
& \leq C h^{2}|\ln h|\left(\left\|\nabla_{s}\left(\Pi_{h}(u)-u_{h}\right)\right\|_{L^{\infty}\left(\mathbb{S}^{2}\right)}+(h+1)\|u\|_{W^{2, \infty}\left(\mathbb{S}^{2}\right)}\right) .
\end{aligned}
$$

Combinando (4.33)-(4.38), tomando o máximo e sabendo que existe um $h_{0} \in \mathbb{R}_{+}$, tal que para $0<h \leq h_{0}$, resulta

$$
\left\|\nabla_{s}\left(\Pi_{h}(u)-u_{h}\right)\right\|_{L^{\infty}\left(\mathbb{S}^{2}\right)} \leq C h|\ln h|\|f\|_{L^{\infty}\left(\mathbb{S}^{2}\right)} .
$$

Como consequência de (4.32) e (4.39) resulta,

$$
\left\|\nabla_{s}\left(u-u_{h}\right)\right\|_{L^{\infty}\left(\mathbb{S}^{2}\right)} \leq C h|\ln h|\left(\|u\|_{W^{2}\left(\mathbb{S}^{2}\right)}+\|f\|_{L^{\infty}\left(\mathbb{S}^{2}\right)}\right) .
$$

Como se queria provar.

Por último, o seguinte resultado estabelecera uma estimativa para a aproximação das soluções em termos da max-norma para a solução exata no espaço $W^{2, \infty}\left(\mathbb{S}^{2}\right) \cap H_{Q}^{1}\left(\mathbb{S}^{2}\right)$.

Teorema 4.6. Sob as hipóteses do Teorema 4.5. Existem constantes $C, h_{0} \in \mathbb{R}_{+}$, que independem de $u$, tal que $0<h \leq h_{0}<1$, tem-se

$$
\left\|\varepsilon_{h}\right\|_{L^{\infty}\left(\mathbb{S}^{2}\right)} \leq C h|\ln h|\|f\|_{L^{\infty}\left(\mathbb{S}^{2}\right)},
$$

$\operatorname{com} \varepsilon_{h}=u-u_{h}$

Demonstração. Procederemos similar ao Teorema 4.5, com um argumento de dualidade. Para y $\in \mathbb{S}^{2}$ fixo, existe uma função suave $\delta^{\mathrm{y}}$ suportada em $\widetilde{\mathrm{T}}_{i j k} \in \widetilde{\mathcal{T}}_{h}$ como na Proposição 4.2. Seja $\hat{G}_{0}^{\mathrm{y}}$, a função discreta de Green que junto à condição (4.22) satisfaz o problema variacional adjunto $(4.25 \mathrm{a})$, i.e.,

$$
\mathcal{A}\left(v, \hat{G}_{0}^{\mathrm{y}}\right)=\left(v, \delta^{\mathrm{y}}\right), \quad \text { para cada } v \in H_{Q}^{1}\left(\mathbb{S}^{2}\right) .
$$

Consideremos também $\hat{G}_{0, h}^{\mathrm{y}}$ a aproximação por elementos finitos de $\hat{G}_{0}^{\mathrm{y}}$, a única solução ao problema (4.26) satisfazendo a condição (1.3). Usando a desigualdade triangular

$$
\left|\left(u-u_{h}\right)(\mathrm{y})\right| \leq\left|\left(u-\Pi_{h}(u)\right)(\mathrm{y})\right|+\left|\left(\Pi_{h}(u)-u_{h}\right)(\mathrm{y})\right| .
$$

Da Proposição 3.10, o primeiro termo do lado direito é limitado por

$$
\left\|u-\Pi_{h}(u)\right\|_{L^{\infty}\left(\mathbb{S}^{2}\right)} \leq C h^{2}\|u\|_{W^{2, \infty}\left(\mathbb{S}^{2}\right)} .
$$

Vamos limitar o termo $\left|\left(\Pi_{h}(u)-u_{h}\right)(\mathrm{y})\right|$. Com efeito, Para $\Pi_{h}(u)-u_{h} \in \widetilde{U}_{h}$, obtemos da linearidade de $\mathcal{A}(\cdot, \cdot)$ que,

$$
\begin{aligned}
\left|\left(\Pi_{h}(u)-u_{h}\right)(\mathrm{y})\right| \leq & C\left|\int_{\mathbb{S}^{2}}\left(\Pi_{h}(u)-u_{h}\right)(\mathrm{x}) \delta^{\mathrm{y}}(\mathrm{x}) d s(\mathrm{x})\right| \\
\leq & C\left|\mathcal{A}\left(\Pi_{h}(u)-u_{h}, \hat{G}_{0}^{\mathrm{y}}\right)\right| \\
\leq & C\left(\left|\mathcal{A}\left(\Pi_{h}(u)-u_{h}, \hat{G}_{0}^{\mathrm{y}}-\hat{G}_{0, h}^{\mathrm{y}}\right)\right|+\left|\mathcal{A}\left(\Pi_{h}(u)-u_{h}, G_{h}^{\mathrm{y}}\right)\right|\right) \\
\leq & C\left(\left|\mathcal{A}\left(\Pi_{h}(u)-u_{h}, \hat{G}_{0}^{\mathrm{y}}-\hat{G}_{0, h}^{\mathrm{y}}\right)\right|+\left|\mathcal{A}\left(\Pi_{h}(u)-u, \hat{G}_{0, h}^{\mathrm{y}}\right)\right|\right. \\
& \left.+\left|\mathcal{A}\left(u-u_{h}, \hat{G}_{0}^{\mathrm{y}}-\hat{G}_{0, h}^{\mathrm{y}}\right)\right|\right) .
\end{aligned}
$$

De (4.26) obtemos $\mathcal{A}\left(\Pi_{h}(u)-u_{h}, \hat{G}_{0}^{\mathrm{y}}-\hat{G}_{0, h}^{\mathrm{y}}\right)=0$. Pela continuidade de $\mathcal{A}(\cdot, \cdot)$ e combinando a 
Proposição (3.10) com a equação (4.27e) temos

$$
\left|\mathcal{A}\left(\Pi_{h}(u)-u, \hat{G}_{0, h}^{\mathrm{y}}\right)\right| \leq C h^{2}|\ln h|\|u\|_{W^{2, \infty}\left(\mathbb{S}^{2}\right)} .
$$

Agora, para tratar a expressão $\left|\mathcal{A}\left(u-u_{h}, \hat{G}_{0}^{\mathrm{y}}-\hat{G}_{0, h}^{\mathrm{y}}\right)\right|$, usamos a linearidade de $\mathcal{A}(\cdot, \cdot)$, somamos e subtraímos os funcionais $\mathcal{A}_{h}^{d}(\cdot, \cdot)$ e $\mathcal{A}_{h}(\cdot, \cdot)$. Isto implicaria que

$$
\begin{aligned}
\left|\mathcal{A}\left(u-u_{h}, \hat{G}_{0, h}^{\mathrm{y}}\right)\right| \leq & \left|\mathcal{A}\left(u, \hat{G}_{0, h}^{\mathrm{y}}\right)-\mathcal{A}_{h}^{d}\left(u_{h}, \mathrm{I}_{h}\left(\hat{G}_{0, h}^{\mathrm{y}}\right)\right)\right|+\left|\mathcal{A}_{h}^{d}\left(u_{h}, \mathrm{I}_{h}\left(\hat{G}_{0, h}^{\mathrm{y}}\right)\right)-\mathcal{A}_{h}\left(u_{h}, \mathrm{I}_{h}\left(\hat{G}_{0, h}^{\mathrm{y}}\right)\right)\right| \\
& +\left|\mathcal{A}_{h}\left(u_{h}, \mathrm{I}_{h}\left(\hat{G}_{0, h}^{\mathrm{y}}\right)\right)-\mathcal{A}\left(u_{h}, \hat{G}_{0, h}^{\mathrm{y}}\right)\right| \\
= & I_{1}+I_{2}+I_{3} .
\end{aligned}
$$

Para $I_{1}$, similar ao Teorema $4.5, \operatorname{com} f \in L^{\infty}\left(\mathbb{S}^{2}\right)$, obtemos a limitação em virtude dos Lemas 4.1 e 4.5 ,

$$
\begin{aligned}
I_{1}=\left|\mathcal{A}\left(u, G_{h}^{\mathrm{y}}\right)-\mathcal{A}_{h}^{d}\left(u_{h}, \mathrm{I}_{h}\left(\hat{G}_{0, h}^{\mathrm{y}}\right)\right)\right|=\left|\left(f, \hat{G}_{0, h}^{\mathrm{y}}-\mathrm{I}_{h}\left(\hat{G}_{0, h}^{\mathrm{y}}\right)\right)\right| & \leq C h\|f\|_{L^{\infty}\left(\mathbb{S}^{2}\right)}\left\|\hat{G}_{0, h}^{\mathrm{y}}\right\|_{W^{1,1}\left(\mathbb{S}^{2}\right)} \\
& \leq C h|\ln h|\|f\|_{L^{\infty}\left(\mathbb{S}^{2}\right)} .
\end{aligned}
$$

Para $I_{2}$, o Lema 3.4 produz

$$
I_{2}=\left|\mathcal{A}_{h}^{d}\left(u_{h}, \mathrm{I}_{h}\left(\hat{G}_{0, h}^{\mathrm{y}}\right)\right)-\mathcal{A}_{h}\left(u_{h}, \mathrm{I}_{h}\left(\hat{G}_{0, h}^{\mathrm{y}}\right)\right)\right| \leq C h^{2}|\ln h|\left\|\nabla_{s} u_{h}\right\|_{L^{\infty}\left(\mathbb{S}^{2}\right)} .
$$

Agora, invocando o Teorema 4.5, existe um $h_{0} \in \mathbb{R}_{+}$tal que para $0<h \leq h_{0}$, tem-se que

$$
\begin{aligned}
\left\|\nabla_{s} u_{h}\right\|_{L^{\infty}\left(\mathbb{S}^{2}\right)} & \leq\left\|\nabla_{s}\left(u-u_{h}\right)\right\|_{L^{\infty}\left(\mathbb{S}^{2}\right)}+\left\|\nabla_{s} u\right\|_{L^{\infty}\left(\mathbb{S}^{2}\right)} \\
& \leq C h|\ln h|\left(\|u\|_{W^{2, \infty}\left(\mathbb{S}^{2}\right)}+\|f\|_{L^{\infty}\left(\mathbb{S}^{2}\right)}\right)+\|u\|_{W^{2, \infty}\left(\mathbb{S}^{2}\right)}
\end{aligned}
$$

Em consequência,

$$
I_{2} \leq C h^{2}|\ln h|\|u\|_{W^{2, \infty}\left(\mathbb{S}^{2}\right)} .
$$

De forma análoga, obteremos uma estimativa para $I_{3}$, usando esta vez o Lema 3.4. Então,

$$
I_{3} \leq C h^{2}|\ln h|\|u\|_{W^{2, \infty}\left(\mathbb{S}^{2}\right)} .
$$

Por último, combinando (4.40)-(4.46) e tomando o máximo, obtemos para $h$ suficientemente pequeno, que

$$
\left\|u-u_{h}\right\|_{L^{\infty}\left(\mathbb{S}^{2}\right)} \leq C h|\ln h|\|f\|_{L^{\infty}\left(\mathbb{S}^{2}\right)} .
$$

Isto encerraria a demonstração.

Para finalizar, podemos obter uma estimativa adicional, agora usando o critério da SCVT, porém alguns modificações nos Lemas 4.2 e 4.3 são necessárias:

Lema 4.7. Suponha que $\widetilde{\mathcal{V}}=\left\{\mathrm{x}_{i}, \widetilde{\mathrm{V}}_{i}\right\}_{i=1}^{N}$ é uma decomposição dual de Voronoï do tipo SCVT de $\mathbb{S}^{2}$ que satisfaz as condições $(2.5 \mathrm{a})-(2.5 \mathrm{c})$ com função de densidade constante $\rho(\mathrm{x})>0$, para cada $\mathrm{x} \in \mathbb{S}^{2}$. Então, para qualquer $w \in W^{2,1}\left(\mathbb{S}^{2}\right) \cap H_{Q}^{1}\left(\mathbb{S}^{2}\right)$, existe uma constante $C \in \mathbb{R}_{+}$, independente de $h$, tal que

$$
\left|\int_{\widetilde{\mathrm{V}}_{i}}\left[w(\mathrm{x})-\widetilde{\Pi}_{h}(w)(\mathrm{x})\right] d s(\mathrm{x})\right| \leq C h^{2}\|w\|_{W^{2,1}\left(\widetilde{\mathrm{V}}_{i}\right)} .
$$

Demonstração. A limitação é dada apenas usando a expansão em série de Taylor e o critério da SCVT como no Lema 4.2.

Lema 4.8. Sejam $f \in W^{1, \infty}\left(\mathbb{S}^{2}\right) \cap H_{Q}^{1}\left(\mathbb{S}^{2}\right)$ e $\widetilde{\mathcal{V}}=\left\{\mathrm{x}_{i}, \widetilde{\mathrm{V}}_{i}\right\}_{i=1}^{N}$ uma decomposição dual de Voronö̈ do tipo $S C V T$ de $\mathbb{S}^{2}$, que satisfaz as condições (2.5a)-(2.5c) com função de densidade constante 


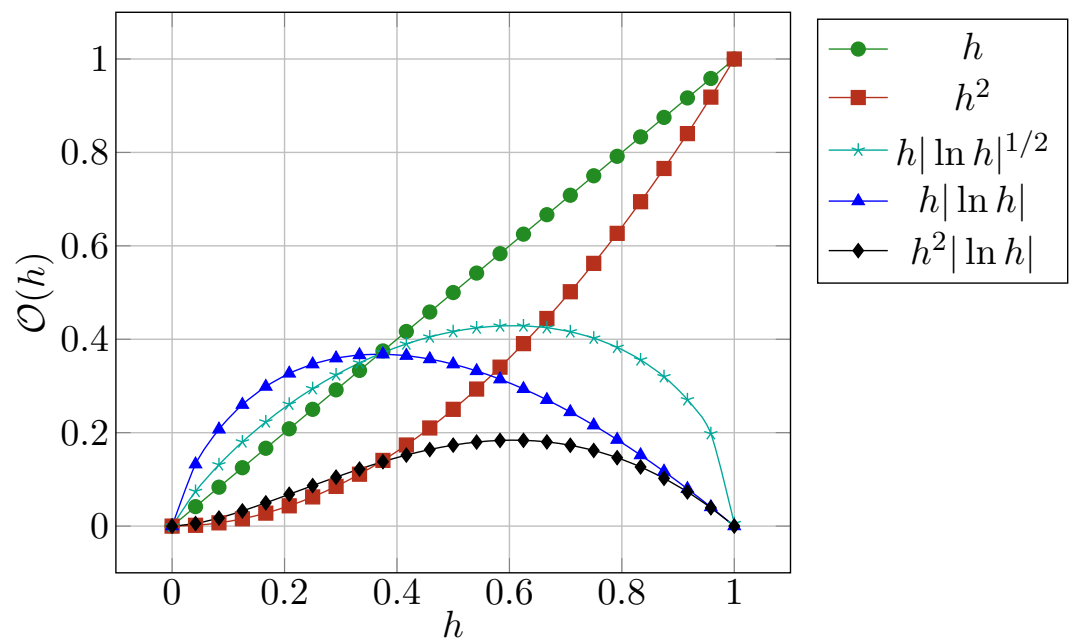

Figura 4.1: Comportamento da ordem de convergência das soluções do Laplaciano na malha esférica icosaédrica na max-norma onde o fator $\mid \ln h^{1 / 2}$ possui ordem $\mathcal{O}\left(h^{-\eta}\right)$ para $\eta \in(0,1)$.

$\rho(\mathrm{x})>0$, para cada $\mathrm{x} \in \mathbb{S}^{2}$. Então, para cada $w \in W^{2,1}\left(\mathbb{S}^{2}\right) \cap H_{Q}^{1}\left(\mathbb{S}^{2}\right)$, existe uma constante $C \in \mathbb{R}_{+}$, independente de $h$, tal que

$$
\left(f, \Pi_{h}(w)-\widetilde{\Pi}_{h}(w)\right) \leq C h^{2}\|f\|_{W^{1, \infty}\left(\mathbb{S}^{2}\right)}\|w\|_{W^{2,1}\left(\mathbb{S}^{2}\right)},
$$

onde $\Pi_{h}$ e $\widetilde{\Pi}_{h}$ são os interpoladores nos espaços $U_{h}$ e $\widetilde{V}_{h}$ respectivamente.

Enunciamos o resultado:

Teorema 4.7. Seja $\widetilde{\mathcal{V}}=\left\{\mathrm{x}_{i}, \widetilde{\mathrm{V}}_{i}\right\}_{i=1}^{N}$ uma decomposição dual de Voronö̈ do tipo SCVT de $\mathbb{S}^{2}$ que satisfaz as condições $(2.5 \mathrm{a})-(2.5 \mathrm{c})$ com função de densidade constante $\rho(\mathrm{x})>0$, para cada $\mathrm{x} \in \mathbb{S}^{2}$. Assuma também que $f \in W^{1, \infty}\left(\mathbb{S}^{2}\right) \cap H_{Q}^{1}\left(\mathbb{S}^{2}\right)$ e que $u \in W^{2, \infty}\left(\mathbb{S}^{2}\right) \cap H_{Q}^{1}\left(\mathbb{S}^{2}\right)$ é a única solução fraca de (1.8). Seja $\mathrm{F}_{i j}^{d}$ o fluxo discreto definido em (3.8), tal que o problema discreto (3.23) tem única solução $u_{h} \in \widetilde{U}_{h}$. Então, existem constantes $C, h_{0} \in \mathbb{R}_{+}$, que independem de $h$, tal que para $0<h \leq h_{0}<1$ tem-se

$$
\left\|\varepsilon_{h}\right\|_{L^{\infty}\left(\mathbb{S}^{2}\right)} \leq C h^{2}|\ln h|\left(\|u\|_{W^{2, \infty}\left(\mathbb{S}^{2}\right)}+\|f\|_{W^{1, \infty}\left(\mathbb{S}^{2}\right)}\right),
$$

$\operatorname{com} \varepsilon_{h}=u-u_{h}$.

Demonstração. A prova é a mesma do Teorema 4.6, agora usando o Lema 4.8 na equação (4.44) junto com $(4.27 \mathrm{c})$ do Lema 4.5, para o ganho da potencia extra de $h$. Então

$$
\begin{aligned}
I_{1}=\left|\mathcal{A}\left(u, G_{h}^{\mathrm{y}}\right)-\mathcal{A}_{h}^{d}\left(u_{h}, \mathrm{I}_{h}\left(G_{h}^{\mathrm{y}}\right)\right)\right|=\left|\left(f, G_{h}^{\mathrm{y}}-\mathrm{I}_{h}\left(G_{h}^{\mathrm{y}}\right)\right)\right| & \leq C h^{2}\|f\|_{W^{1, \infty}\left(\mathbb{S}^{2}\right)}\left\|G^{\mathrm{y}}\right\|_{W^{2,1}\left(\mathbb{S}^{2}\right)} \\
& \leq C h^{2}|\ln h|\|f\|_{W^{1, \infty}\left(\mathbb{S}^{2}\right)} .
\end{aligned}
$$

Portanto obtemos o desejado.

Observação 4.3. O Teorema 4.5 é suficiente para garantir uma estimativa de ordem sub-linear na $W^{1, \infty}$-norma com o critério da SCVT, pois não há um ganho adicional, a limitação fica restrita à aproximação da Proposição 3.10, mais precisamente da equação (4.32).

Por último, a Figura 4.1 ilustra as diferentes ordens de convergência das soluções dos esquemas de volumes finitos usual usando decomposições Voronoï-Delaunay. 


\subsection{Experimentos numéricos}

Nesta seção ilustraremos um exemplo numérico para a aproximação do Laplaciano considerando a construção recursiva apresentada no Seção 2.2 para os três tipos NOPT, SCVT e HR95. Em particular, para a SCVT consideraremos a função de densidade constante $\rho=1$. A fim de verificar a estimativa dos erros $\varepsilon_{h}$, usaremos o exemplo dado por Heikes e Randall (1995b), com solução exata $u$ em coordenadas geográficas latitude-longitude $(\phi, \theta)$ (vide Apêndice B),

$$
u(\phi, \theta)=\cos (\theta) \cos ^{4}(\phi),
$$

e seu respectivo Laplaciano,

$$
\begin{array}{r}
f(\phi, \theta)=-\cos (\theta) \cos ^{2}(\theta)\left[\cos ^{2}(\phi)-4 \sin (\phi) \cos (\phi) \sin (\phi) \cos (\phi)\right. \\
\left.-12 \cos ^{2}(\phi)+16 \cos ^{2}(\phi) \cos ^{2}(\phi)\right] / \cos ^{2}(\phi) .
\end{array}
$$

Na Tabela 4.1 mostra a estimativas do erro da solução do problema (4.48) com as $H^{1}, L^{2}$, max e $W^{1, \infty}$-norma nos três tipos de malhas com diferentes níveis de refinamento. Observamos que a taxa de convergência $p$ é próxima de um na malha NOPT para a $H^{1}$-norma e a $W^{1, \infty}$-norma confirmando os resultados dos Teoremas 4.1 e 4.5. Tanto na $L^{2}$-norma como na max-norma a NOPT apresenta uma ordem de convergência maior da estimada nos Teoremas 4.2 e 4.4/4.6. Não obstante, as estimativas nessas normas são independentes da estimativa do erro de truncamento em contraste com o mostrado na Seção 3.2. Em particular, a diferença entre os problemas variacionais dos MVF e MEFL aplicados à solução exata junto com à decomposição Voronoï-Delaunay em geral são um fator dominante na obtenção da taxa ideal de convergência.

Por outro lado, observe-se que a taxa de convergência $p$ para a SCVT é próxima de dois na $L^{2}$-norma confirmando a estimativa do Teorema 4.3. O mesmo comportamento é mostrado na max-norma confirmando o Teorema 4.7. Note-se ademais um melhor comportamento no erro da $H^{1}$-norma nas duas otimizações, i.e., existe um grau de superconvergência na aproximação dos gradientes. Por enquanto, não existe algum critério teórico que comprove esse comportamento e em consequência gera um bom desafio de pesquisa futura.

Para finalizar este capítulo, deixamos na Tabela 4.2 algumas das contribuições mais relevantes da literatura. 


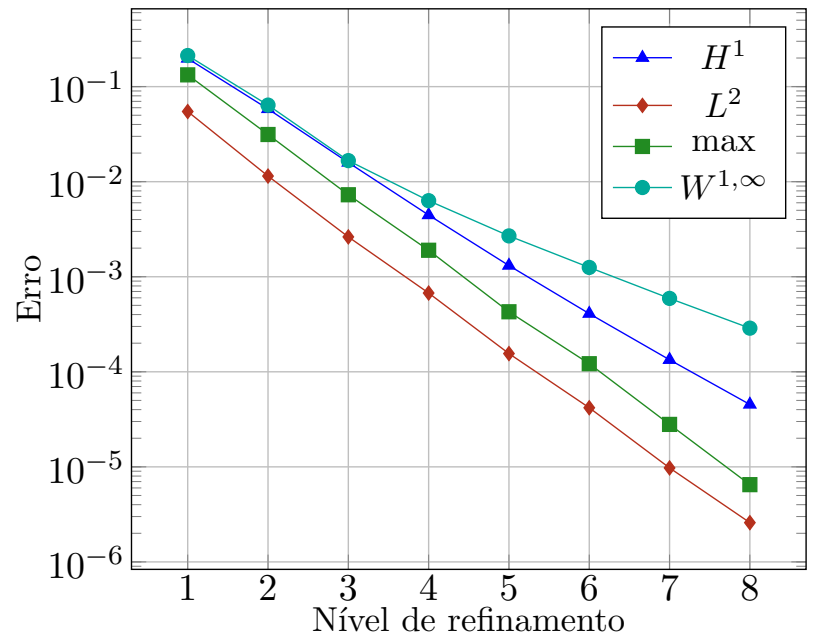

(a) $N O P T$

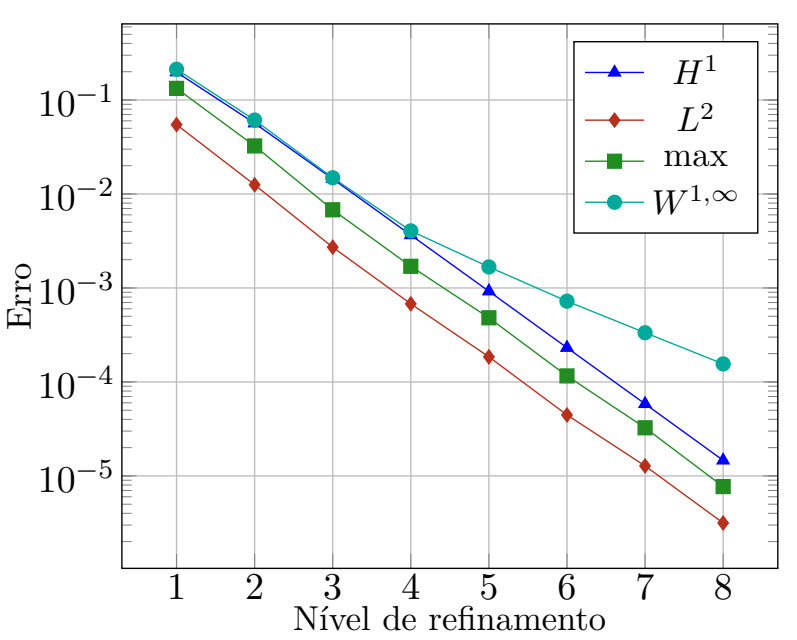

(b) $S C V T$

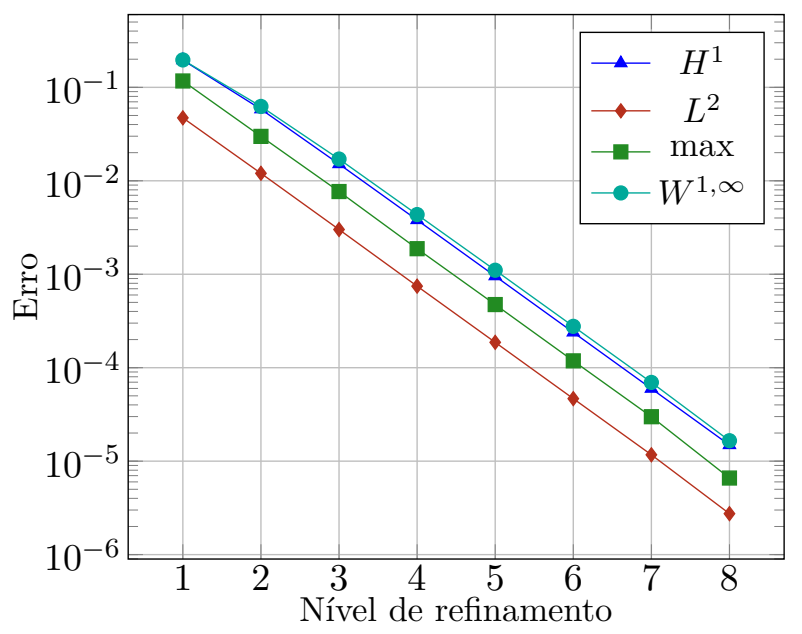

(c) HR95

Figura 4.2: Representação gráfica dos valores da Tabela 4.1 para o erro das soluções aproximadas do Laplaciano nas normas estudadas para as malhas NOPT, SCVT e HR95 com níveis de refinamento de 1 (42 vértices) até 8 (655362 vértices). 


\begin{tabular}{|c|c|c|c|c|c|c|}
\hline \multicolumn{7}{|c|}{ ERRO DA SOLUÇÃO NA $H_{h}^{1}$-NORMA E TAXA DE CONVERGÊNCIA $p$} \\
\hline \multirow[b]{2}{*}{ NÍVEL } & \multicolumn{2}{|c|}{ NOPT } & \multicolumn{2}{|c|}{ SCVT } & \multicolumn{2}{|c|}{ HR95 } \\
\hline & $\left\|\varepsilon_{h}\right\|_{1, h}$ & $p$ & $\left\|\varepsilon_{h}\right\|_{1, h}$ & $p$ & $\left\|\varepsilon_{h}\right\|_{1, h}$ & $p$ \\
\hline 1 & $1.977 \mathrm{E}-01$ & - & $1.977 \mathrm{E}-01$ & - & $1.958 \mathrm{E}-01$ & - \\
\hline 2 & $5.827 \mathrm{E}-02$ & $1.763 \mathrm{E}+00$ & $5.660 \mathrm{E}-02$ & $1.805 \mathrm{E}+00$ & $5.839 \mathrm{E}-02$ & $1.745 \mathrm{E}+00$ \\
\hline 3 & $1.591 \mathrm{E}-02$ & $1.873 \mathrm{E}+00$ & $1.448 \mathrm{E}-02$ & $1.967 \mathrm{E}+00$ & $1.511 \mathrm{E}-02$ & $1.951 \mathrm{E}+00$ \\
\hline 4 & $4.457 \mathrm{E}-03$ & $1.836 \mathrm{E}+00$ & $3.657 \mathrm{E}-03$ & $1.985 \mathrm{E}+00$ & $3.808 \mathrm{E}-03$ & $1.988 \mathrm{E}+00$ \\
\hline 5 & $1.311 \mathrm{E}-03$ & $1.766 \mathrm{E}+00$ & $9.228 \mathrm{E}-04$ & $1.987 \mathrm{E}+00$ & $9.545 \mathrm{E}-04$ & $1.996 \mathrm{E}+00$ \\
\hline 6 & 4.091E-04 & $1.680 \mathrm{E}+00$ & $2.314 \mathrm{E}-04$ & $1.996 \mathrm{E}+00$ & $2.388 \mathrm{E}-04$ & $1.999 \mathrm{E}+00$ \\
\hline 7 & $1.338 \mathrm{E}-04$ & $1.612 \mathrm{E}+00$ & $5.841 \mathrm{E}-05$ & $1.986 \mathrm{E}+00$ & $5.971 \mathrm{E}-05$ & $2.000 \mathrm{E}+00$ \\
\hline \multirow[t]{2}{*}{8} & $4.531 \mathrm{E}-05$ & $1.563 \mathrm{E}+00$ & $1.464 \mathrm{E}-05$ & $1.996 \mathrm{E}+00$ & $1.489 \mathrm{E}-05$ & $2.003 \mathrm{E}+00$ \\
\hline & $\left\|\varepsilon_{h}\right\|_{0, h}$ & $p$ & $\left\|\varepsilon_{h}\right\|_{0, h}$ & $p$ & $\left\|\varepsilon_{h}\right\|_{0, h}$ & $p$ \\
\hline 1 & $5.472 \mathrm{E}-02$ & - & $5.472 \mathrm{E}-02$ & - & $4,716 \mathrm{E}-02$ & - \\
\hline 2 & $1.146 \mathrm{E}-02$ & $2.255 \mathrm{E}+00$ & $1.252 \mathrm{E}-02$ & $2.128 \mathrm{E}+00$ & $1.202 \mathrm{E}-02$ & $1.972 \mathrm{E}+00$ \\
\hline 3 & $2.625 \mathrm{E}-03$ & $2.127 \mathrm{E}+00$ & $2.716 \mathrm{E}-03$ & $2.204 \mathrm{E}+00$ & $3.011 \mathrm{E}-03$ & $1.996 \mathrm{E}+00$ \\
\hline 4 & $6.739 \mathrm{E}-04$ & $1.962 \mathrm{E}+00$ & $6.778 \mathrm{E}-04$ & $2.003 \mathrm{E}+00$ & $7.458 \mathrm{E}-04$ & $2.014 \mathrm{E}+00$ \\
\hline 5 & $1.555 \mathrm{E}-04$ & $2.115 \mathrm{E}+00$ & $1.850 \mathrm{E}-04$ & $1.873 \mathrm{E}+00$ & $1.868 \mathrm{E}-04$ & $1.997 \mathrm{E}+00$ \\
\hline 6 & $4.185 \mathrm{E}-05$ & $1.894 \mathrm{E}+00$ & $4.456 \mathrm{E}-05$ & $2.053 \mathrm{E}+00$ & 4.667E-05 & $2.001 \mathrm{E}+00$ \\
\hline 7 & $9.745 \mathrm{E}-06$ & $2.103 \mathrm{E}+00$ & $1.279 \mathrm{E}-05$ & $1.801 \mathrm{E}+00$ & $1.170 \mathrm{E}-05$ & $1.996 \mathrm{E}+00$ \\
\hline \multirow[t]{2}{*}{8} & $2.588 \mathrm{E}-06$ & $1.913 \mathrm{E}+00$ & $3.156 \mathrm{E}-06$ & $2.019 \mathrm{E}+00$ & $2.743 \mathrm{E}-06$ & $2.093 \mathrm{E}+00$ \\
\hline & $\left\|\varepsilon_{h}\right\|_{\infty, h}$ & $p$ & $\left\|\varepsilon_{h}\right\|_{\infty, h}$ & $p$ & $\left\|\varepsilon_{h}\right\|_{\infty, h}$ & $p$ \\
\hline 1 & $1.330 \mathrm{E}-01$ & - & $1.330 \mathrm{E}-01$ & - & 1.170E-01 & - \\
\hline 2 & $3.136 \mathrm{E}-02$ & $2.085 \mathrm{E}+00$ & $3.245 \mathrm{E}-02$ & $2.035 \mathrm{E}+00$ & $2.987 \mathrm{E}-02$ & $1.970 \mathrm{E}+00$ \\
\hline 3 & $7.286 \mathrm{E}-03$ & $2.106 \mathrm{E}+00$ & 6.790E-03 & $2.257 \mathrm{E}+00$ & 7.677E-03 & $1.960 \mathrm{E}+00$ \\
\hline 4 & $1.900 \mathrm{E}-03$ & $1.939 \mathrm{E}+00$ & $1.700 \mathrm{E}-03$ & $1.998 \mathrm{E}+00$ & $1.877 \mathrm{E}-03$ & $2.032 \mathrm{E}+00$ \\
\hline 5 & $4.280 \mathrm{E}-04$ & $2.150 \mathrm{E}+00$ & $4.815 \mathrm{E}-04$ & $1.820 \mathrm{E}+00$ & 4.739E-04 & $1.986 \mathrm{E}+00$ \\
\hline 6 & $1.216 \mathrm{E}-04$ & $1.815 \mathrm{E}+00$ & $1.159 \mathrm{E}-04$ & $2.055 \mathrm{E}+00$ & $1.184 \mathrm{E}-04$ & $2.001 \mathrm{E}+00$ \\
\hline 7 & $2.796 \mathrm{E}-05$ & $2.121 \mathrm{E}+00$ & $3.252 \mathrm{E}-05$ & $1.834 \mathrm{E}+00$ & $2.989 \mathrm{E}-05$ & $1.985 \mathrm{E}+00$ \\
\hline \multirow[t]{2}{*}{8} & $6.497 \mathrm{E}-06$ & $2.106 \mathrm{E}+00$ & $7.704 \mathrm{E}-06$ & $2.077 \mathrm{E}+00$ & $6.599 \mathrm{E}-06$ & $2.180 \mathrm{E}+00$ \\
\hline & $\mid \varepsilon_{h} \|_{W^{1, \infty}\left(\mathbb{S}^{2}\right)}$ & $p$ & $\left\|\varepsilon_{h}\right\|_{W^{1, \infty}\left(\mathbb{S}^{2}\right)}$ & $p$ & $\mid \varepsilon_{h} \|_{W^{1, \infty}\left(\mathbb{S}^{2}\right)}$ & $p$ \\
\hline 1 & $2.124 \mathrm{E}-01$ & - & $2.124 \mathrm{E}-01$ & - & $1.965 \mathrm{E}-01$ & - \\
\hline 2 & 6.391E-02 & $1.733 \mathrm{E}+00$ & 6.119E-02 & $1.796 \mathrm{E}+00$ & $6.248 \mathrm{E}-02$ & $1.653 \mathrm{E}+00$ \\
\hline 3 & $1.666 \mathrm{E}-02$ & $1.939 \mathrm{E}+00$ & $1.489 \mathrm{E}-02$ & $2.039 \mathrm{E}+00$ & $1.709 \mathrm{E}-02$ & $1.870 \mathrm{E}+00$ \\
\hline 4 & $6.313 \mathrm{E}-03$ & $1.400 \mathrm{E}+00$ & $4.050 \mathrm{E}-03$ & $1.878 \mathrm{E}+00$ & $4.351 \mathrm{E}-03$ & $1.974 \mathrm{E}+00$ \\
\hline 5 & $2.689 \mathrm{E}-03$ & $1.231 \mathrm{E}+00$ & $1.674 \mathrm{E}-03$ & $1.275 \mathrm{E}+00$ & $1.104 \mathrm{E}-03$ & $1.978 \mathrm{E}+00$ \\
\hline 6 & $1.253 \mathrm{E}-03$ & $1.102 \mathrm{E}+00$ & $7.232 \mathrm{E}-04$ & $1.211 \mathrm{E}+00$ & $2.771 \mathrm{E}-04$ & $1.995 \mathrm{E}+00$ \\
\hline 7 & $5.919 \mathrm{E}-04$ & $1.082 \mathrm{E}+00$ & $3.341 \mathrm{E}-04$ & $1.114 \mathrm{E}+00$ & $6.960 \mathrm{E}-05$ & $1.993 \mathrm{E}+00$ \\
\hline 8 & $2.878 \mathrm{E}-04$ & $1.040 \mathrm{E}+00$ & $1.556 \mathrm{E}-04$ & $1.102 \mathrm{E}+00$ & $1.655 \mathrm{E}-05$ & $2.072 \mathrm{E}+00$ \\
\hline
\end{tabular}

Tabela 4.1: Estimativas do erro da solução $\varepsilon_{h}$ nas $H^{1}, L^{2}$, $\max$ e $W^{1, \infty}$-normas com a sua respectiva taxa de convergência p obtidas do Laplaciano discreto (3.10) para a função (4.47) nas malhas NOPT, SCVT e HR95. 


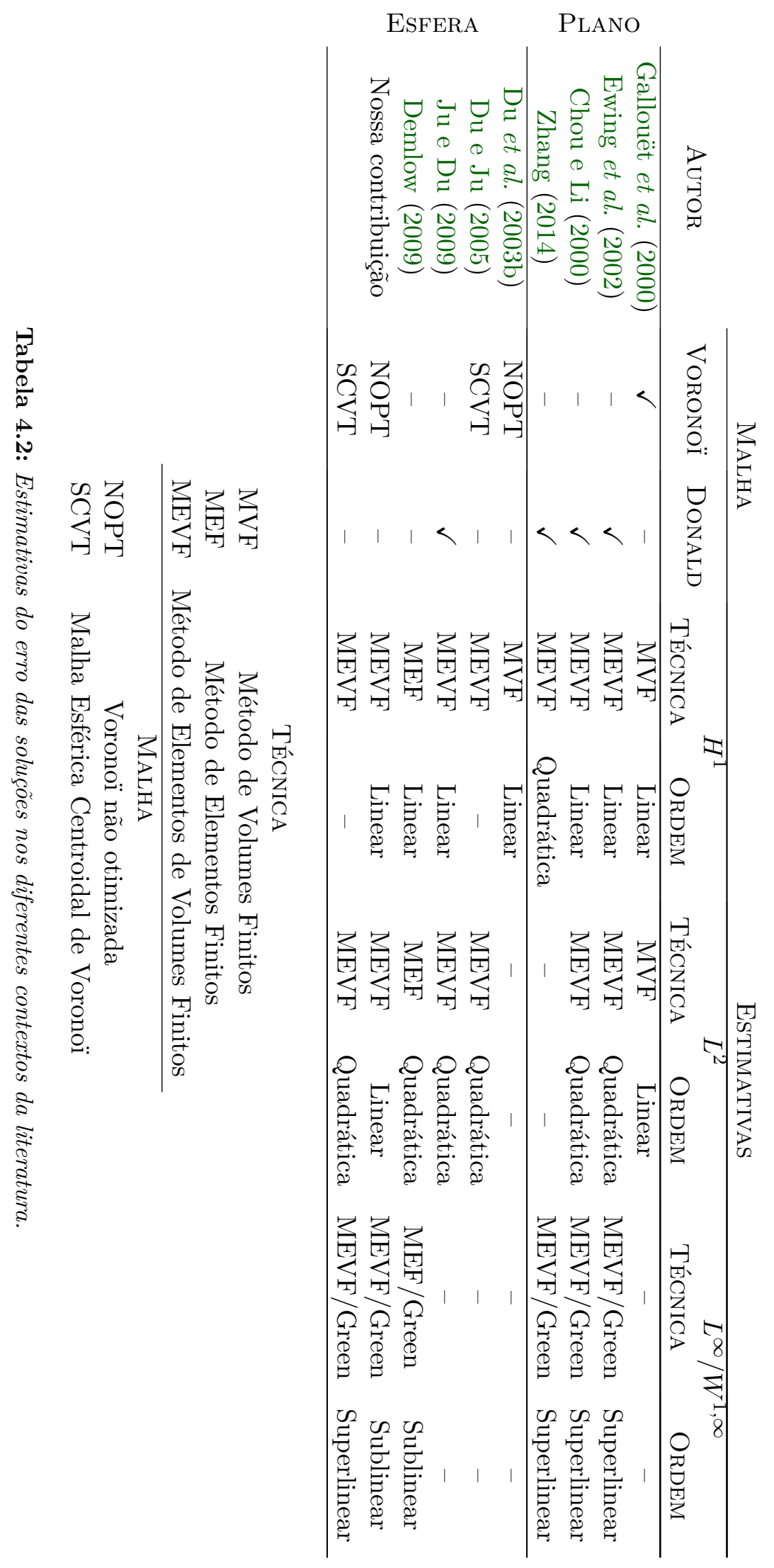




\section{Conclusões}

Nos últimos anos, o uso de malhas mais isotrópicas na dinâmica de fluidos na esfera se tornou de maior interesse, em especial devido à necessidade de uso eficiente de paralelismo em computadores de alta performance. Neste trabalho consideramos malhas geradas a partir de um icosaedro inscrito em $\mathbb{S}^{2}$, conhecidas como malhas esféricas geodésicas icosaédricas, as quais permitem uma distribuição mais uniforme dos vértices na superfície esférica e definir, com base no princípio de dualidade, decomposições Voronoï-Delaunay em $\mathbb{S}^{2}$. Além disso, foram consideradas duas otimizações referentes à qualidade geométrica de malha, a SCVT proposta por Du et al. (2003a) e a HR95 proposta por Heikes e Randall (1995b). Características geométricas dessas decomposições tais como: área, posicionamento dos centroides das células, relação entre as arestas das decomposições primal/dual e distorção das células têm sido estudadas em detalhe nos trabalhos de Gregory et al. (2008); Miura e Kimoto (2005) e Peixoto (2013).

Apresentamos a discretização usual do problema de Poisson na esfera $\mathbb{S}^{2}$ através dos Métodos de Volumes Finitos (MVF). A discretização parte da forma integral da equação, com o uso do teorema de Gauss em volumes de controle. O procedimento utiliza uma estimação dos fluxos nos pontos médios das arestas do volume finito (as células de Voronoï) que garante uma aproximação de segunda ordem em relação ao tamanho de malha. Em seguida, uma outra aproximação via um esquema de diferença finita centrada foi definida para o fluxo nos pontos médios das arestas duais. Apesar do esquema possuir características importantes como a conservação local, i.e., o fluxo numérico é conservado de uma célula para suas vizinhas, a não uniformidade das células acarreta um esquema assintoticamente inconsistente (Heikes e Randall, 1995b; Ringler, 2003). Não obstante, como evidenciado em experimentos numéricos, o erro das soluções comporta-se "melhor" que o erro de truncamento. Esta característica é conhecida como supra-convergência e foi definida em vários trabalhos anteriores, como por exemplo Diskin e Thomas (2010); Kreiss et al. (1986); Manteuffel e White (1986); Pascal (2007). Ainda hoje, há grande dificuldade para se obter estimativas da ordem de convergência dos MVF em malhas não uniformes, utilizando as técnicas usuais da análise dos Métodos das Diferenças Finitas (MDF). Para contornar essa situação e conforme a literatura (Chou e Ye, 2007; Ewing et al., 2002; Li et al., 2000; Lin et al., 2013), é habitual considerar o MVF como um Método de Elementos Finitos Lineares (MEFL). A formulação tem sido definida associando um par de espaços adequados de aproximação associados à decomposição Voronoï-Delaunay em $\mathbb{S}^{2}$. Com base nisso, estabelecemos uma expressão para a estabilidade usando uma forma variacional discreta do MVF. Estimamos também certos erros geométricos causados pela aproximação da esfera unitária $\mathbb{S}^{2}$ por uma sequência de poliedros $\mathbf{S}_{h}$ com $h$ indo para zero. Isto com o fim de determinar as limitações essenciais das diferenças entre as formas variacionais construídas para cada método, i.e., dos MVF e MEFL.

Com isso em mente, nós focamos nas estimativas de erro das soluções. É conveniente notar que, apesar de existir toda uma teoria desenvolvida para problemas no plano (Chen et al., 2002; Chou e Li, 2000; Chou e Ye, 2007; Chou et al., 2003; Jianguo e Shitong, 1998; Zhang, 2014), a abordagem fica muito atrás quando os MVF usam decomposições Voronoï-Delaunay, tanto no plano como em $\mathbb{S}^{2}$. Uma primeira linha de contribuições desta tese começa da expressão da coercividade da forma variacional de (1.4). Após, deduzido o relacionamento entre os MVF e MEFL, enunciamos estimativas clássicas nas $H^{1}$ e $L^{2}$-normas com ordem de convergência um, um pouco diferentes das apresentadas em Du et al. (2003b), no sentido que usamos os procedimentos padrão dos MEFL. Estimativas de convergência de ordem 2 na norma $L^{2}$ são obtidas se a decomposição VoronoïDelaunay é otimizada pelo critério da SCVT, como apresentado por Du e Ju (2005). Neste caso, 
mostramos uma modificação da prova, pois a regularidade na solução exata é excessiva em contraste com a obtida pelos MEFL. Usando a ideia de Ewing et al. (2002), a qual adaptamos para as malhas SCVT, exigimos uma mínima regularidade no termo fonte, a função $f$, pertence ao espaço $H_{Q}^{1}\left(\mathbb{S}^{2}\right)$ e assim obter uma diminuição da regularidade na solução exata, i.e., estar no espaço $H^{2}\left(\mathbb{S}^{2}\right) \cap H_{Q}^{1}\left(\mathbb{S}^{2}\right)$.

Fazendo uso do recente desenvolvimento das técnicas da análise pontuais dos MEF em superfícies (Demlow, 2009; Demlow e Dziuk, 2007; Kovács e Power Guerra, 2018; Kröner, 2017) a principal contribuição desta tese centrou-se na obtenção de estimativas fracas na max e $W^{1, \infty}$ norma. Seguindo os trabalhos mencionados, representamos uma forma variacional do problema de Laplace usando funções de Green regularizadas como funções auxiliares do problema adjunto. A exposição dos resultados de convergência, os Teoremas 4.4-4.6 têm ordem sublinear e o Teorema 4.7 ordem superlinear (quase quadrática) em relação às potencias do tamanho de malha $h$. Não obstante, aparece um fator logarítmico, que não pode ser evitado, causado pelo uso das funções lineares por partes na decomposição primal de Delaunay. Esperamos que os resultados apresentados aqui, ainda possam ser melhorados, quem sabe usando técnicas como tem sido mostrado em domínios Euclidianos por Rannacher e Scott (1982); Schatz e Wahlbin (1995) e Schatz (1998) que aprimorem a ordem junto com técnicas de relaxamento nas condições geométricas da decomposição Voronoï-Delaunay.

Além disso, a estrutura da análise nesta tese pode ser estendida para problemas lineares de convecção-difusão, tendo em vista alguns cuidados adicionais, e para problemas de difusão como

$$
-\nabla_{s} \cdot\left(\kappa(\mathrm{x}) \nabla_{s} u(\mathrm{x})\right)=f(\mathrm{x}), \quad \text { para cada } \mathrm{x} \in \mathbb{S}^{2},
$$

onde $\kappa$ é um coeficiente da difusão usualmente descontinuo.

De forma geral, diversos requerimentos são necessários para obter resultados próximos aos proporcionados numericamente, independente da malha esférica icosaédrica escolhida. Concluí-se então que, foram estabelecidas estimativas pontuais satisfatórias dos erros das soluções construídas pelo esquema de volumes finitos, embora haja evidências numéricas de que a ordem de convergência ainda possa ser melhorada.

\section{Possíveis extensões deste trabalho}

1. Estabelecer as estimativas de erro a posteriori para MVF Adaptativos nas malhas SCVT com refinamento local como nos trabalhos de Bonito e Demlow (2019); Demlow e Dziuk (2007); Ju et al. (2009). Este é um aspecto importante a ser considerado no desenvolvimento de modelos de previsão do tempo.

2. Análise de estimativas post-processing de soluções do MVF para obter o seu gradiente tangencial (recuperação do gradiente) usando a SCVT com refinamento local.

3. Análise de convergência nas normas usuais para as malhas de tipo HR95 usando a metodologia descrita nesta tese.

4. Fazer uma extensão adequada da análise de convergência nas $L^{2}$ e max-norma dos trabalhos de Lv e Li (2010) e Nie et al. (2017) para esfera cubada, i.e., a geração da malha esférica a partir de um hexaedro inscrito em $\mathbb{S}^{2}$.

5. Implementação da decomposição dual de Donald (usando baricentros dos triângulos) na esfera e adaptar a análise de convergência neste caso. 


\section{Apêndice A}

\section{Erro de consistência} 3.

Neste apêndice damos as provas das Proposições sobre o erro de truncamento dadas no Capítulo

\section{A.1 Uma condição de consistência fraca}

Como foi discutido na Seção 3.2 o erro de truncamento não é consistente (no sentido das diferenças finitas). A perda dessa ordem de precisão é devida, em princípio às aproximações da integral de linha na aresta da célula e após da derivada normal via um esquema de diferenças finitas centradas no ponto médio das arestas duais. Apesar disso, esse problema de inconsistência na aproximação pode ser corrigido incorporando uma condição alternativa conhecida na literatura como consistência fraca (Coudière et al., 1999; Faille, 1992). Vamos descrever as hipóteses que colocam-se na análise da consistência fraca:

Hipótese A.1 (Ortogonalidade). A decomposição Voronoï-Delaunay é uma malha admissível (no sentido de Gallouët et al., 2000, pp. 1936, Eymard et al., 2000, pp. 757) de $\mathbb{S}^{2}$ que satisfaz as condições (2.5a)-(2.5c) e possui ortogonalidade entre as arestas primal/dual.

Pode-se construir uma outra malha esférica (vide Du et al., 2003b), da seguinte maneira: considere os vértices $\mathrm{x}_{i}$ e $\mathrm{x}_{j}$ vizinhos que atendem a relação entre as distâncias

$$
\frac{1}{2} \mathrm{~d}\left(\mathrm{x}_{i}, \mathrm{x}_{j}\right) \leq\left\|\mathrm{x}_{i}-\mathrm{x}_{j}\right\| \leq \mathrm{d}\left(\mathrm{x}_{i}, \mathrm{x}_{j}\right)
$$

Além disso, $\widetilde{\Gamma}_{i j}=\operatorname{cl}\left(\widetilde{\mathrm{V}}_{i}\right) \cap \operatorname{cl}\left(\widetilde{\mathrm{V}}_{j}\right)$ é a aresta comum das células de $\widetilde{\mathrm{V}}_{i}$ e $\widetilde{\mathrm{V}}_{j}$ associadas aos vértices $\mathrm{x}_{i}$ e $\mathrm{x}_{j}$ respectivamente com $m\left(\widetilde{\Gamma}_{i j}\right)>0$, de acordo à Seção 1.1. Então, denotemos por $\widetilde{\mathrm{D}}_{i j}^{(n)} \mathrm{o}$ triângulo esférico formado pela aresta $\widetilde{\Gamma}_{i j}$ e o vértice $\mathrm{x}_{n}$ para $n=i, j$, e $\widetilde{\mathrm{D}}_{i j}$ o diamante esférico formado pela união dos triângulos $\widetilde{\mathrm{D}}_{i j}^{(n)}$, com $n \in\{i, j\}$, i.e.,

$$
\widetilde{\mathrm{D}}_{i j}=\widetilde{\mathrm{D}}_{i j}^{(i)} \cup \widetilde{\mathrm{D}}_{i j}^{(j)}
$$

Ademais, as suas áreas superficiais são definidas respectivamente como

$$
\begin{aligned}
S\left(\widetilde{\mathrm{D}}_{i j}\right) & =\frac{1}{2} m\left(\widetilde{\Gamma}_{i j}\right) \mathrm{d}\left(\mathrm{x}_{i}, \mathrm{x}_{j}\right), \\
S\left(\widetilde{\mathrm{D}}_{i j}^{(n)}\right) & =\frac{1}{2} m\left(\widetilde{\Gamma}_{i j}\right) \mathrm{d}\left(\mathrm{x}_{n}, \mathrm{x}\right), \quad \text { para cada } \mathrm{x} \in \widetilde{\Gamma}_{i j} \text { e } n \in\{i, j\} .
\end{aligned}
$$

Definimos também o conjunto

$$
\mathrm{D}_{i j}^{(n)}=\left\{\mathrm{x}_{n}+t\left(\mathrm{x}-\mathrm{x}_{n}\right): \mathrm{x} \in \widetilde{\Gamma}_{i j}, t \in[0,1]\right\}, \quad \operatorname{com} n \in\{i, j\},
$$

tal que $\widetilde{\mathrm{D}}_{i j}^{(n)}$ seja a sua projeção radial sobre a superfície esférica. Observe que, para $n \in\{i, j\}$ as regiões $\widetilde{\mathrm{D}}_{i j}^{(n)}$ e $\mathrm{D}_{i j}^{(n)}$ estão contidas no conjunto $\Omega$ como definido nas provas das Proposições 3.1-3.4. 


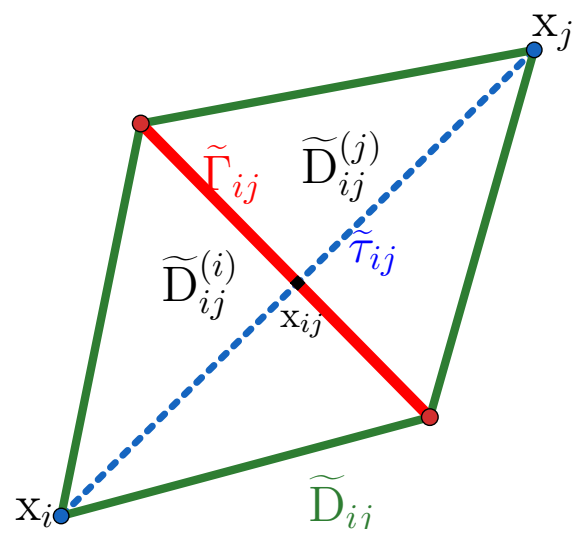

Figura A.1: Representação geométrica do losango $\widetilde{\mathrm{D}}_{i j}=\widetilde{\mathrm{D}}_{i j}^{(i)} \cup \widetilde{\mathrm{D}}_{i j}^{(j)}$, cujas diagonais estão definidas pela aresta dual $\widetilde{\Gamma}_{i j}$ e a primal $\widetilde{\tau}_{i j}$ com ponto médio $\mathrm{x}_{i j}$.

Hipótese A.2 (Consistência dos fluxos). Dada $u: \mathbb{S}^{2} \rightarrow \mathbb{R}$ uma função de classe $C^{4}$. Consideremos $\widetilde{u}$ a função constante por pedaços cujas componentes são ou os valores exatos de u ou uma aproximação o mais perto dela, digamos por exemplo os seus valores médios em cada célula.

Então, dizemos que o fluxo contínuo $\mathrm{F}_{i j}^{c}(u)$ possui uma aproximação consistente $\mathrm{F}_{i j}^{d}(\widetilde{u})$ de ordem menor ou igual ao tamanho de malha $h$. Em outras palavras, os fluxos numéricos $\mathrm{F}^{d}$ são aproximações consistentes dos fluxos exatos $\mathrm{F}^{c}$, se substituímos os valores aproximados $u_{h}$ por os valores de $\widetilde{u}$ (Eymard et al., 2000). Desejá-se provar que o operador discreto (3.11) satisfaz a propriedade das aproximações consistentes nos seus fluxos, mais precisamente:

Proposição A.1 (Aproximação consistente). Sejam $\widetilde{\mathrm{V}}_{i}$ uma célula da decomposição dual de Voronoï que satisfaz as condições $(2.5 \mathrm{a})-(2.5 \mathrm{c})$ e $u \in C^{4}\left(\mathbb{S}^{2}\right) \cap H_{Q}^{1}\left(\mathbb{S}^{2}\right)$ a única solução ao problema (1.4). Considere também os fluxos continuo $\mathrm{F}_{i j}^{c}$ e discreto $\mathrm{F}_{i j}^{d}$ como definidos em (3.3) e (3.10) respectivamente. Então, existe uma constante $C \in \mathbb{R}_{+}$independente do parâmetro $h$, tal que

$$
\left|\mathrm{F}_{i j}^{c}(u)-\mathrm{F}_{i j}^{d}(\widetilde{u})\right| \leq C h^{2},
$$

onde $\widetilde{u}$ denota os valores exatos de u nos vértices da malha. Dizemos que $\mathrm{F}_{i j}^{d}(\widetilde{u})$ é uma aproximação consistente do fluxo $\mathrm{F}_{i j}^{c}(u)$ a través da aresta dual $\widetilde{\Gamma}_{i j}$.

Demonstração. A prova é bastante simples, pois segue diretamente da combinação das Proposições 3.2 e 3.4 junto à desigualdade triangular. Além disso, a constante irá depender apenas das segundas derivadas de $u$. Nesse sentido, temos

$$
\mathrm{F}_{i j}^{d}(\widetilde{u})=-m\left(\widetilde{\Gamma}_{i j}\right) \nabla_{s} u\left(\mathrm{~m}_{i j}\right) \cdot \overrightarrow{\mathrm{n}}_{\mathrm{x}, \widetilde{\Gamma}_{i j}}+R_{i j}
$$

onde $\left|R_{i j}\right| \leq C h^{2} \max _{\mathrm{x} \in\left(\widetilde{\mathrm{V}}_{i} \cup \widetilde{\mathrm{V}}_{j}\right)}\left|H\left(u^{\Omega}\right)(\mathrm{x})\right|$. Invocando a Hipótese A.1, denotamos o fluxo por,

$$
\mathrm{F}_{i j}^{d}(\widetilde{u})=m\left(\widetilde{\Gamma}_{i j}\right)\left[-\nabla_{s} u\left(\mathrm{~m}_{i j}\right) \cdot \overrightarrow{\mathrm{n}}_{\mathrm{x}, \widetilde{\Gamma}_{i j}}+\epsilon_{i j}\right],
$$

onde $\left|\epsilon_{i j}\right| \leq h \max _{\mathrm{x} \in\left(\widetilde{\mathrm{V}}_{i} \cup \widetilde{\mathrm{V}}_{j}\right)}\left|H\left(u^{\Omega}\right)(\mathrm{x})\right|$.

No que segue, sabemos que a propriedade de conservação é uma característica natural no esquema dos volumes finitos. Denotamos agora, o erro de truncamento por

$$
\widetilde{\xi}_{h}=\mathrm{L}_{s, h}(\widetilde{u})-f_{h} .
$$




\section{A.1.1 Prova da Proposição 3.5}

São assumidas as Hipóteses A.1 e A.2.

Demonstração da Proposição 3.5. Dada $\widetilde{\mathrm{D}}_{i j}$ a região esférica como em (A.1). Basta provar para $u \in C^{2}\left(\widetilde{\mathrm{D}}_{i j}\right)$. Consideremos $u^{\Omega}$ a extensão de $u$ em $\Omega_{i j}=\left\{\mathrm{x}^{*} \in \Omega: \mathcal{P}\left(\mathrm{x}^{*}\right) \in \widetilde{\mathrm{D}}_{i j}\right\}$. Então, usando o Teorema de Taylor até ordem dois de $u^{\Omega}$. Para $\mathrm{x} \in \widetilde{\Gamma}_{i j}$, temos

$$
u^{\Omega}\left(\mathrm{x}_{i}\right)-u^{\Omega}(\mathrm{x})=\nabla u^{\Omega}(\mathrm{x}) \cdot\left(\mathrm{x}_{i}-\mathrm{x}\right)+\int_{0}^{1} H\left(u^{\Omega}\right)\left(\mathrm{x}_{i}+t\left(\mathrm{x}-\mathrm{x}_{i}\right)\right)\left(\mathrm{x}_{i}-\mathrm{x}\right) \cdot\left(\mathrm{x}_{i}-\mathrm{x}\right) d t .
$$

Similarmente,

$$
u^{\Omega}\left(\mathrm{x}_{j}\right)-u^{\Omega}(\mathrm{x})=\nabla u^{\Omega}(\mathrm{x}) \cdot\left(\mathrm{x}_{j}-\mathrm{x}\right)+\int_{0}^{1} H\left(u^{\Omega}\right)\left(\mathrm{x}_{j}+t\left(\mathrm{x}-\mathrm{x}_{j}\right)\right)\left(\mathrm{x}_{j}-\mathrm{x}\right) \cdot\left(\mathrm{x}_{j}-\mathrm{x}\right) d t
$$

onde $H\left(u^{\Omega}\right)(\mathrm{x})$ denota a matriz Hessiana com valores de $u^{\Omega}$ no ponto x. Subtraindo (A.5) de (A.6) e integrando em relação a $\widetilde{\Gamma}_{i j}$ resulta

$$
m\left(\widetilde{\Gamma}_{i j}\right)\left[u^{\Omega}\left(\mathrm{x}_{j}\right)-u^{\Omega}\left(\mathrm{x}_{i}\right)\right]=\int_{\widetilde{\Gamma}_{i j}} \nabla u^{\Omega}(\mathrm{x}) \cdot\left(\mathrm{x}_{j}-\mathrm{x}_{i}\right) d \gamma(\mathrm{x})+R_{i j}^{(i)}+R_{i j}^{(j)},
$$

onde

$$
R_{i j}^{(n)}=\int_{\widetilde{\Gamma}_{i j}} \int_{0}^{1} H\left(u^{\Omega}\right)\left(\mathrm{x}_{n}+t\left(\mathrm{x}-\mathrm{x}_{n}\right)\right)\left(\mathrm{x}_{n}-\mathrm{x}\right) \cdot\left(\mathrm{x}_{n}-\mathrm{x}\right) d t d \gamma(\mathrm{x}), \quad \operatorname{com} n \in\{i, j\}
$$

Observe-se que o vetor normal $\vec{n}_{\mathrm{x}, \widetilde{\Gamma}_{i j}}$ é paralelo a $\overrightarrow{\mathrm{x}_{i} \mathrm{x}_{j}}$ conforme enunciado na Subseção 2.2.2, então

$$
\mathrm{x}_{i}-\mathrm{x}_{j}=\left\|\mathrm{x}_{i}-\mathrm{x}_{j}\right\| \overrightarrow{\mathrm{n}}_{\mathrm{x}, \widetilde{\Gamma}_{i j}} .
$$

Ademais, da definição do gradiente tangencial e o fato de $\vec{n}_{\mathbb{S}^{2}, \mathrm{x}}$ e $\overrightarrow{\mathrm{n}}_{\mathrm{x}, \widetilde{\Gamma}_{i j}}$ ser ortogonais para $\mathrm{x} \in \mathbb{S}^{2}$ resulta,

$$
\nabla_{s} u(\mathrm{x}) \cdot \overrightarrow{\mathrm{n}}_{\mathrm{x}, \widetilde{\Gamma}_{i j}}=\nabla u^{\Omega}(\mathrm{x}) \cdot \overrightarrow{\mathrm{n}}_{\mathrm{x}, \widetilde{\Gamma}_{i j}}-\left(\nabla u^{\Omega}(\mathrm{x}) \cdot \overrightarrow{\mathrm{n}}_{\mathbb{S}^{2}, \mathrm{x}}\right)\left(\overrightarrow{\mathrm{n}}_{\mathbb{S}^{2}, \mathrm{x}} \cdot \overrightarrow{\mathrm{n}}_{\mathrm{x}, \widetilde{\Gamma}_{i j}}\right)=\nabla u^{\Omega}(\mathrm{x}) \cdot \overrightarrow{\mathrm{n}}_{\mathrm{x}, \widetilde{\Gamma}_{i j}} \cdot
$$

Em primeiro lugar, dividindo por $m\left(\widetilde{\Gamma}_{i j}\right)\left\|\mathrm{x}_{j}-\mathrm{x}_{i}\right\|$, e para simplificar a notação, vamos definir a diferença dos fluxo por $\widetilde{\xi}_{i j}(u) \operatorname{com} u(\mathrm{x})=u^{\Omega}(\mathrm{x})$ para cada $\mathrm{x} \in \mathbb{S}^{2}$, o que implica

$$
\widetilde{\xi}_{i j}(u)=\frac{1}{m\left(\widetilde{\Gamma}_{i j}\right)}\left[\mathrm{F}_{i j}^{d}(\widetilde{u})-\mathrm{F}_{i j}^{c}(u)\right]=\frac{1}{m\left(\widetilde{\Gamma}_{i j}\right)\left\|\mathrm{x}_{j}-\mathrm{x}_{i}\right\|}\left[R_{i j}^{(i)}+R_{i j}^{(j)}\right] .
$$

Agora, basta limitar o lado direito da identidade acima. Veja que $\left\|\mathrm{x}_{j}-\mathrm{x}_{i}\right\| \leq h$ e que $\frac{1}{2} \mathrm{~d}\left(\mathrm{x}_{i}, \mathrm{x}_{j}\right) \leq$ $\left\|\mathrm{x}_{j}-\mathrm{x}_{i}\right\|$. Então, para $n \in\{i, j\}$, segue

$$
\begin{aligned}
\frac{1}{m\left(\widetilde{\Gamma}_{i j}\right)\left\|\mathrm{x}_{j}-\mathrm{x}_{i}\right\|} R_{i j}^{(n)} & \leq \frac{h_{n}^{2}}{m\left(\widetilde{\Gamma}_{i j}\right)\left\|\mathrm{x}_{j}-\mathrm{x}_{i}\right\|} \int_{\widetilde{\Gamma}_{i j}} \int_{0}^{1}\left|H\left(u^{\Omega}\right)\left(\mathrm{x}_{n}+t\left(\mathrm{x}-\mathrm{x}_{n}\right)\right)\right| d t d \gamma(\mathrm{x}) \\
& \leq \frac{2 h_{n}^{2}}{m\left(\widetilde{\Gamma}_{i j}\right) \mathrm{d}\left(\mathrm{x}_{i}, \mathrm{x}_{j}\right)} \int_{\widetilde{\Gamma}_{i j}} \int_{0}^{1}\left|H\left(u^{\Omega}\right)\left(\mathrm{x}_{n}+t\left(\mathrm{x}-\mathrm{x}_{n}\right)\right)\right| d t d \gamma(\mathrm{x}) .
\end{aligned}
$$

O passo a seguir será estimar a integral no lado direito de (A.8), porém alguns cuidados são necessários. Da Seção 1.1, a mudança de variável e a relação entre as medidas das integrais estão bem definidas, logo

$$
\mathrm{d}\left(\mathrm{x}_{i}, \mathrm{x}_{j}\right) \int_{\widetilde{\Gamma}_{i j}} \int_{0}^{1} d t d \gamma(\mathrm{x})=\mathrm{d}\left(\mathrm{x}_{i}, \mathrm{x}_{j}\right) \int_{\widetilde{\Gamma}_{i j}} d \gamma(\mathrm{x})=\mathrm{d}\left(\mathrm{x}_{i}, \mathrm{x}_{j}\right) \int_{\widetilde{\Gamma}_{i j}} d \gamma(\mathrm{x})=\mathrm{d}\left(\mathrm{x}_{i}, \mathrm{x}_{j}\right) m\left(\widetilde{\Gamma}_{i j}\right) .
$$


Para a última expressão, usamos a desigualdade triangular para $\mathrm{x} \in \widetilde{\Gamma}_{i j}$, logo

$$
\mathrm{d}\left(\mathrm{x}_{i}, \mathrm{x}_{j}\right) m\left(\widetilde{\Gamma}_{i j}\right) \leq 2\left\|\mathrm{x}_{j}-\mathrm{x}_{i}\right\| m\left(\widetilde{\Gamma}_{i j}\right) \leq 4\left\|\mathrm{x}-\mathrm{x}_{n}\right\| m\left(\widetilde{\Gamma}_{i j}\right), \quad \text { para } n \in\{i, j\} .
$$

Conforme a definição da região $\mathrm{D}_{i j}^{(n)}$ em (A.3), com área superficial dada por $S\left(\mathrm{D}_{i j}^{(n)}\right)=\frac{1}{2} m\left(\widetilde{\Gamma}_{i j}\right) \| \mathrm{x}-$ $\mathrm{x}_{n} \|$, obtemos

$$
\frac{1}{2} \mathrm{~d}\left(\mathrm{x}_{i}, \mathrm{x}_{j}\right) m\left(\widetilde{\Gamma}_{i j}\right) \leq S\left(\mathrm{D}_{i j}^{(n)}\right)=\int_{\mathrm{D}_{i j}^{(n)}} d s\left(\mathrm{x}^{*}\right) .
$$

Em consequência obtemos a expressão,

$$
\int_{\widetilde{\Gamma}_{i j}} \int_{0}^{1} d t d \gamma(\mathrm{x}) \leq \frac{1}{\mathrm{~d}\left(\mathrm{x}_{i}, \mathrm{x}_{j}\right)} \int_{\mathrm{D}_{i j}^{(n)}} d s\left(\mathrm{x}^{*}\right) .
$$

Por outro lado, da relação

$$
S\left(\mathrm{D}_{i j}^{(n)}\right) \leq \frac{1}{2} m\left(\widetilde{\Gamma}_{i j}\right) \mathrm{d}\left(\mathrm{x}, \mathrm{x}_{n}\right),
$$

onde $\mathrm{d}\left(\mathrm{x}, \mathrm{x}_{n}\right) \leq h_{n}$, para cada $n=i, j$. Segue então que

$$
S\left(\mathrm{D}_{i j}^{(n)}\right) \leq \frac{1}{2} m\left(\widetilde{\Gamma}_{i j}\right) \mathrm{d}\left(\mathrm{x}, \mathrm{x}_{n}\right) \leq \frac{1}{2} m\left(\widetilde{\Gamma}_{i j}\right) h_{n} \leq \frac{1}{4 \sigma} m\left(\widetilde{\Gamma}_{i j}\right) \mathrm{d}\left(\mathrm{x}_{i}, \mathrm{x}_{j}\right) .
$$

Na última expressão do lado direito, usamos a definição do parâmetro de regularidade $\sigma$ conforme definido em (2.2) da Subseção 2.2.2. Agora, retomando nosso cálculo da integral, para $\mathrm{x}^{*}=$ $\mathrm{x}_{n}+t\left(\mathrm{x}-\mathrm{x}_{n}\right)$, invocando a desigualdade de Cauchy-Schwarz junto ao tratamento feito acima, produzimos a estimativa,

$$
\begin{aligned}
\frac{1}{m\left(\widetilde{\Gamma}_{i j}\right)\left\|\mathrm{x}_{j}-\mathrm{x}_{i}\right\|} R_{i j}^{(n)} & \leq \frac{2 h_{n}^{2}}{m\left(\widetilde{\Gamma}_{i j}\right) \mathrm{d}\left(\mathrm{x}_{i}, \mathrm{x}_{j}\right)} \int_{\widetilde{\Gamma}_{i j}} \int_{0}^{1}\left|H\left(u^{\Omega}\right)\left(\mathrm{x}_{n}+t\left(\mathrm{x}-\mathrm{x}_{n}\right)\right)\right| d t d \gamma(\mathrm{x}) \\
& \leq \frac{2 h_{n}^{2}}{m\left(\widetilde{\Gamma}_{i j}\right) \mathrm{d}\left(\mathrm{x}_{i}, \mathrm{x}_{j}\right)^{2}} \int_{\mathrm{D}_{i j}^{(n)}}\left|H\left(u^{\Omega}\right)\left(\mathrm{x}^{*}\right)\right| d s\left(\mathrm{x}^{*}\right) \\
& \leq \frac{2 h_{n}^{2} \sqrt{S\left(\mathrm{D}_{i j}^{(n)}\right)}}{m\left(\widetilde{\Gamma}_{i j}\right) \mathrm{d}\left(\mathrm{x}_{i}, \mathrm{x}_{j}\right)^{2}}\left(\int_{\mathrm{D}_{i j}^{(n)}}\left|H\left(u^{\Omega}\right)\left(\mathrm{x}^{*}\right)\right|^{2} d s\left(\mathrm{x}^{*}\right)\right)^{1 / 2},
\end{aligned}
$$

onde

$$
\left|H\left(u^{\Omega}\right)(\mathrm{x})\right|^{2}=\sum_{n, m=1}^{3}\left|\partial_{n} \partial_{m} u^{\Omega}(\mathrm{x})\right|^{2},
$$

representa a norma de Frobenius da Hessiana. Segundo Du et al. (2003b, Lema 1, pp. 3941), existe uma constante $C \in \mathbb{R}_{+}$, tal que

$$
\left(\int_{\mathrm{D}_{i j}^{(n)}}\left|H\left(u^{\Omega}\right)(\mathrm{x})\right|^{2} d s(\mathrm{x})\right)^{1 / 2} \leq C\|u\|_{H^{2}\left(\widetilde{\mathrm{D}}_{i j}^{(n)}\right)}, \quad \text { para cada } n \in\{i, j\} .
$$

Da relação (A.9) e o fato da malha ser quase-uniforme, em particular, $C h \leq \mathrm{d}\left(\mathrm{x}_{i}, \mathrm{x}_{j}\right)$, obtemos

$$
\frac{1}{m\left(\widetilde{\Gamma}_{i j}\right)\left\|\mathrm{x}_{j}-\mathrm{x}_{i}\right\|} R_{i j}^{(n)} \leq C \frac{2 h_{n}}{\sigma \sqrt{\sigma m\left(\widetilde{\Gamma}_{i j}\right) \mathrm{d}\left(\mathrm{x}_{i}, \mathrm{x}_{j}\right)}}\|u\|_{H^{2}\left(\widetilde{\mathrm{D}}_{i j}^{(n)}\right)}, \quad \text { com } n \in\{i, j\} .
$$


Combinando (A.7) e (A.10) com $n=i, j$, tem-se

$$
\begin{aligned}
\left|\widetilde{\xi}_{i j}(u)\right| & \leq C\left(\frac{2 h_{i}}{\sigma \sqrt{\sigma m\left(\widetilde{\Gamma}_{i j}\right) \mathrm{d}\left(\mathrm{x}_{i}, \mathrm{x}_{j}\right)}}\|u\|_{H^{2}\left(\widetilde{\mathrm{D}}_{i j}^{(i)}\right)}+\frac{2 h_{j}}{\sigma \sqrt{\sigma m\left(\widetilde{\Gamma}_{i j}\right) \mathrm{d}\left(\mathrm{x}_{i}, \mathrm{x}_{j}\right)}}\|u\|_{H^{2}\left(\widetilde{\mathrm{D}}_{i j}^{(j)}\right)}\right) \\
& \leq C \frac{h}{\sigma \sqrt{\sigma m\left(\widetilde{\Gamma}_{i j}\right) \mathrm{d}\left(\mathrm{x}_{i}, \mathrm{x}_{j}\right)}}\|u\|_{H^{2}\left(\widetilde{\mathrm{D}}_{i j}\right)}
\end{aligned}
$$

Invocamos um argumento de densidade: sejam $u \in H^{2}\left(\widetilde{\mathrm{D}}_{i j}\right)$ e a sequência $\left\{u_{m}\right\}_{m \geq 1}$ em $C^{2}\left(\operatorname{cl}\left(\widetilde{\mathrm{D}}_{i j}\right)\right)$ tal que $u_{m} \longrightarrow u$ na $H^{2}\left(\widetilde{\mathrm{D}}_{i j}\right)$-norma. Conforme Brezis (2010); Hebey (1996), a esfera $\mathbb{S}^{2}$ é uma variedade compacta, então garante que $u_{m}$ converge para $u$ uniformemente em $H^{2}\left(\widetilde{\mathrm{D}}_{i j}\right)$ e o fato que $\nabla_{s} u_{m}(\mathrm{x}) \cdot \overrightarrow{\mathrm{n}}_{\mathrm{x}, \widetilde{\Gamma}_{i j}}$ converge para $\nabla_{s} u(\mathrm{x}) \cdot \overrightarrow{\mathrm{n}}_{\mathrm{x}, \widetilde{\Gamma}_{i j}}$ em $L^{1}\left(\widetilde{\mathrm{D}}_{i j}\right)$.

Para finalizar, dada a área superficial $S\left(\widetilde{\mathrm{D}}_{i j}\right)=\frac{1}{2} m\left(\widetilde{\Gamma}_{i j}\right) \mathrm{d}\left(\mathrm{x}_{i}, \mathrm{x}_{j}\right)$ e o fato que cada célula $\widetilde{\mathrm{V}}_{i}$ pode ser decomposta nos triângulos esféricos formados pelo vértice associado $\mathrm{x}_{i}$ e cada aresta $\widetilde{\Gamma}_{i j}$ da sua fronteira, então temos que a área superficial é dada por

$$
S\left(\widetilde{\mathrm{V}}_{i}\right)=\sum_{j \in \Lambda(i)} S\left(\widetilde{\mathrm{D}}_{i j}^{(i)}\right), \quad \text { para } i=1, \ldots, N
$$

Temos para o erro de consistência $\widetilde{\xi}_{h}$ na $L^{2}$-norma discreta que,

$$
\left\|\widetilde{\xi}_{h}\right\|_{0, h}^{2}=\sum_{i=1}^{N} S\left(\widetilde{\mathrm{V}}_{i}\right)\left|\widetilde{\xi}_{i}\right|^{2}=\sum_{i=1}^{N} \sum_{j \in \Lambda(i)} S\left(\widetilde{\mathrm{D}}_{i j}^{(i)}\right)\left|\widetilde{\xi}_{i j}(u)\right|^{2}
$$

Da relação $S\left(\widetilde{\mathrm{D}}_{i j}^{(i)}\right) \leq m\left(\widetilde{\Gamma}_{i j}\right) \mathrm{d}\left(\mathrm{x}_{i}, \mathrm{x}_{j}\right)$, a limitação desejada segue imediatamente de (A.11), i.e.,

$$
\begin{aligned}
\left\|\xi_{h}\right\|_{0, h}^{2} & \leq C \sum_{i=1}^{N} \sum_{j \in \Lambda(i)} S\left(\widetilde{\mathrm{D}}_{i j}^{(i)}\right)\left|\frac{h}{\sigma \sqrt{\sigma m\left(\widetilde{\Gamma}_{i j}\right) \mathrm{d}\left(\mathrm{x}_{i}, \mathrm{x}_{j}\right)}}\|u\|_{H^{2}\left(\widetilde{\mathrm{D}}_{i j}\right)}\right|^{2} \\
& \leq C h^{2} \sum_{i=1}^{N} \sum_{j \in \Lambda(i)} \frac{1}{2} m\left(\widetilde{\Gamma}_{i j}\right) \mathrm{d}\left(\mathrm{x}_{i}, \mathrm{x}_{j}\right)\left|\frac{\|u\|_{H^{2}\left(\widetilde{\mathrm{D}}_{i j}\right)}}{\sigma \sqrt{\sigma m\left(\widetilde{\Gamma}_{i j}\right) \mathrm{d}\left(\mathrm{x}_{i}, \mathrm{x}_{j}\right)}}\right|^{2} \\
& \leq C \frac{h^{2}}{\sigma^{3}} \sum_{i=1}^{N} \sum_{j \in \Lambda(i)}\|u\|_{H^{2}\left(\widetilde{\mathrm{D}}_{i j}^{(i)}\right)} \leq C h^{2}\|u\|_{H^{2}\left(\mathbb{S}^{2}\right)}^{2} .
\end{aligned}
$$

Portanto, $\left\|\xi_{h}\right\|_{0, h} \leq C h\|u\|_{H^{2}\left(\mathbb{S}^{2}\right)}$, com $C$ dependendo unicamente de $\sigma$. Isto encerra a demonstração.

O resultado a seguir é um resultado direto da proposição 3.5 e a propriedade de estabilidade 3.13 .

Teorema A.1. Seja $\widetilde{\mathcal{V}}=\left\{\mathrm{x}_{i}, \widetilde{\mathrm{V}}_{i}\right\}_{i=1}^{N}$ a decomposição dual de Voronö̈ de $\mathbb{S}^{2}$ (NOPT) que satisfaz as condições (2.5a)-(2.5c). Assuma que a única solução u de (1.8) pertence ao espaço $H^{2}\left(\mathbb{S}^{2}\right) \cap H_{Q}^{1}\left(\mathbb{S}^{2}\right)$. Seja $\mathrm{F}_{i j}^{d}$ o fluxo discreto definido em (3.3), tal que o problema discreto (3.23) tem única solução $u_{h} \in U_{h}$. Então, existe uma constante $C \in \mathbb{R}_{+}$, que só depende do parâmetro $\sigma$, tal que

$$
\left\|\varepsilon_{h}\right\|_{1, h} \leq C h\|u\|_{H^{2}\left(\mathbb{S}^{2}\right)}
$$

onde $\varepsilon_{h}=\Pi_{h}(u)-u_{h}$ é a função de malha do erro da solução e $\Pi_{h}(u)$ é a interpolação da função $u$ nas locaçôes da decomposição primal $\widetilde{\mathcal{T}}_{h}$.

Demonstração. Seja $\varepsilon_{h}=\Pi_{h}(u)-u_{h}$. Então, da Proposição 3.12 e a ortogonalidade no funcional 
$\mathcal{A}_{h}^{d}(\cdot, \cdot)$

$$
\mathcal{A}_{h}^{d}\left(u-\Pi_{h}(u), \mathrm{I}_{h}\left(v_{h}\right)\right)=0, \quad \text { para cada } v_{h} \in \widetilde{U}_{h} .
$$

Obtemos

$$
\left\|\varepsilon_{h}\right\|_{1, h}^{2}=\left\|\Pi_{h}(u)-u_{h}\right\|_{1, h}^{2} \leq C\left|\mathcal{A}_{h}^{d}\left(\Pi_{h}(u)-u_{h}, \mathrm{I}_{h}\left(\varepsilon_{h}\right)\right)\right|=\left|\mathcal{A}_{h}^{d}\left(u-u_{h}, \mathrm{I}_{h}\left(\varepsilon_{h}\right)\right)\right| .
$$

Da definição da forma bilinear obtemos

$$
\mathcal{A}_{h}^{d}\left(u-u_{h}, \mathrm{I}_{h}\left(\varepsilon_{h}\right)\right)=\sum_{i=1}^{N} \sum_{j \in \Lambda(i)}\left[\mathrm{F}_{i j}^{c}(u)-\mathrm{F}_{i j}^{d}(u)\right] \varepsilon_{h}\left(\mathrm{x}_{i}\right)=\sum_{i=1}^{N} \sum_{j \in \Lambda(i)} m\left(\widetilde{\Gamma}_{i j}\right) \xi_{i j}(u) \varepsilon_{h}\left(\mathrm{x}_{i}\right),
$$

onde $\widetilde{\xi}_{i j}(u)$ é definido por

$$
\widetilde{\xi}_{i j}(u)=\frac{1}{m\left(\widetilde{\Gamma}_{i j}\right)}\left[\mathrm{F}_{i j}^{c}(u)-\mathrm{F}_{i j}^{d}(u)\right] .
$$

Pela conservação discreta do esquema resulta,

$$
\begin{aligned}
\mathcal{A}_{h}^{d}\left(u-u_{h}, \mathrm{I}_{h}\left(\varepsilon_{h}\right)\right) & =\frac{1}{2} \sum_{i=1}^{N} \sum_{j \in \Lambda(i)} m\left(\widetilde{\Gamma}_{i j}\right) \widetilde{\xi}_{i j}(u)\left[\varepsilon_{h}\left(\mathrm{x}_{j}\right)-\varepsilon_{h}\left(\mathrm{x}_{i}\right)\right] \\
& =\frac{1}{2} \sum_{i=1}^{N} \sum_{j \in \Lambda(i)} m\left(\widetilde{\Gamma}_{i j}\right)\left\|\mathrm{x}_{j}-\mathrm{x}_{i}\right\| \widetilde{\xi}_{i j}(u) \frac{\varepsilon_{h}\left(\mathrm{x}_{j}\right)-\varepsilon_{h}\left(\mathrm{x}_{i}\right)}{\left\|\mathrm{x}_{j}-\mathrm{x}_{i}\right\|} .
\end{aligned}
$$

A estimativa $\left\|\mathrm{x}_{j}-\mathrm{x}_{i}\right\| \leq \mathrm{d}\left(\mathrm{x}_{i}, \mathrm{x}_{j}\right)$ gera,

$$
\mathcal{A}_{h}^{d}\left(u-u_{h}, \mathrm{I}_{h}\left(\varepsilon_{h}\right)\right) \leq \frac{1}{2} \sum_{i=1}^{N} \sum_{j \in \Lambda(i)} m\left(\widetilde{\Gamma}_{i j}\right) \mathrm{d}\left(\mathrm{x}_{i}, \mathrm{x}_{j}\right) \widetilde{\xi}_{i j}(u) \frac{\varepsilon_{h}\left(\mathrm{x}_{j}\right)-\varepsilon_{h}\left(\mathrm{x}_{i}\right)}{\left\|\mathrm{x}_{j}-\mathrm{x}_{i}\right\|} .
$$

Agora, da desigualdade de Cauchy-Schwarz obtemos

$$
\begin{aligned}
\mathcal{A}_{h}^{d}\left(u-u_{h}, \mathrm{I}_{h}\left(\varepsilon_{h}\right)\right) \leq & \left(\sum_{i=1}^{N} \sum_{j \in \Lambda(i)} \frac{1}{2} m\left(\widetilde{\Gamma}_{i j}\right) \mathrm{d}\left(\mathrm{x}_{i}, \mathrm{x}_{j}\right)\left|\widetilde{\xi}_{i j}(u)\right|^{2}\right)^{1 / 2} \\
& \times\left(\sum_{i=1}^{N} \sum_{j \in \Lambda(i)} \frac{1}{2} m\left(\widetilde{\Gamma}_{i j}\right) \mathrm{d}\left(\mathrm{x}_{i}, \mathrm{x}_{j}\right)\left|\frac{\varepsilon_{h}\left(\mathrm{x}_{j}\right)-\varepsilon_{h}\left(\mathrm{x}_{i}\right)}{\left\|\mathrm{x}_{j}-\mathrm{x}_{i}\right\|}\right|^{2}\right)^{1 / 2} .
\end{aligned}
$$

Conforme a Proposição 3.5, existe uma constante $C \in \mathbb{R}_{+}$, que depende somente de $\sigma$, temos a estimativa

$$
\mathcal{A}_{h}^{d}\left(u-u_{h}, \mathrm{I}_{h}\left(\varepsilon_{h}\right)\right) \leq C h\|u\|_{H^{2}\left(\mathbb{S}^{2}\right)}\left\|\varepsilon_{h}\right\|_{1, h},
$$

$\log 0$

$$
\left\|\varepsilon_{h}\right\|_{1, h}^{2} \leq C h\|u\|_{H^{2}\left(\mathbb{S}^{2}\right)}\left\|\varepsilon_{h}\right\|_{1, h} .
$$

Portanto segue a conclusão (A.11).

Estimativas na mesma norma discreta e da mesma ordem na esfera podem ser consultadas no trabalho de Du et al. (2003b).

\section{A.1.2 Prova da Proposição 3.6}

Introduzimos algumas definições para a demostração.

Definição A.1 (Funções simples). Uma função simples é uma combinação de funções características de conjuntos mensuráveis. Mais precisamente o espaço $\left(\mathbb{S}^{2}, \mathrm{~d}\right)$ é um espaço mensurável. Sejam $\left\{\mathrm{x}_{i}, \widetilde{\mathrm{V}}_{i}\right\}_{i=1}^{N}$ uma sequencia de conjunto mensuráveis disjuntos e $\left\{\alpha_{i}\right\}_{i=1}^{N}$ uma 
sequencia de números reais. Uma função simples é uma função $s: \mathbb{S}^{2} \rightarrow \mathbb{R}$ da forma

$$
s(\mathrm{x})=\sum_{i=1}^{N} \alpha_{i} \chi_{i}(\mathrm{x})
$$

onde $\chi_{i}$ denota a função característica da região $\widetilde{\mathrm{V}}_{i}$,

$$
\chi_{i}(\mathrm{x}):= \begin{cases}1, & \text { se } \mathrm{x} \in \widetilde{\mathrm{V}}_{i}, \\ 0, & \text { se } \mathrm{x} \notin \widetilde{\mathrm{V}}_{i} .\end{cases}
$$

Nessa ordem de ideias, podemos considerar $\mathbb{S}^{2}$ como um espaço mensurável, com a medida da área superficial $S$. O fato de uma função simples $s$ ser anulada fora de um conjunto de medidas finitas significa que

$$
S\left(\mathrm{x} \in \mathbb{S}^{2}: s(\mathrm{x}) \neq 0\right)<\infty,
$$

de fato $S\left(\widetilde{\mathrm{V}}_{i}\right)<\infty$, logo para $1 \leq p<\infty$

$$
\|s\|_{L^{p}\left(\mathbb{S}^{2}\right)}=\sum_{i=1}^{N}\left|\alpha_{i}\right|^{p} S\left(\widetilde{\mathrm{V}}_{i}\right) .
$$

Assim, $s \in L^{p}\left(\mathbb{S}^{2}\right)$ se e somente se anula-se fora de um conjunto de medida finita. Podemos enunciar o próximo resultado. Cabe aclarar que a demonstração referida não é original do autor, mas se é considerado como um exercício clássico na análise funcional e na teoria da medida.

Lema A.1 (Rudin, 2006). Para $1 \leq p<\infty$, o conjunto das funçôes simples mensuráveis, as quais se anulam fora de um conjunto de medida finita é denso em $L^{p}\left(\mathbb{S}^{2}\right)$.

Demonstração. Seja $1 \leq p<\infty$ e $f \in L^{p}\left(\mathbb{S}^{2}\right)$. A demonstrar que dado um $\epsilon<0$, existe uma função $s$ simples mensurável, a qual é anulada fora de um conjunto de medida finita tal que $\|f-s\|_{L^{p}\left(\mathbb{S}^{2}\right)}<\epsilon$.

Consideremos primeiro $f \geq 0$. Sabemos que existe uma sequencia $\left\{s_{m}\right\}_{m \geq 1}$ de funções simples não negativas e mensuráveis tal que $s_{m} \longrightarrow f$ pontualmente em $\mathbb{S}^{2}$, dado que $0 \leq s_{m} \leq f$ para cada $m \in \mathbb{N}$ e $f \in L^{p}\left(\mathbb{S}^{2}\right)$, temos $s_{m} \in L^{p}\left(\mathbb{S}^{2}\right)$, em consequência $s_{m}$ se anulam fora de um conjunto de medida finita. Observe que

$$
\lim _{m \rightarrow \infty}\left|s_{m}-f\right|^{p}=0, \quad \text { em } \mathbb{S}^{2}
$$

e

$$
\left|s_{m}-f\right|^{p} \leq\left(\left|s_{m}\right|+|f|\right)^{p} \leq(2|f|)^{p} .
$$

Dado que $f \in L^{p}\left(\mathbb{S}^{2}\right)$, implica que $2^{p}|f|^{p} \in L^{1}\left(\mathbb{S}^{2}\right)$. Invocando o Teorema de convergência dominada de Lebesgue (vide por exemplo Brezis, 2010) temos que

$$
\lim _{m \rightarrow \infty} \int_{\mathbb{S}^{2}}\left|s_{m}(\mathrm{x})-f(\mathrm{x})\right|^{p} d s(\mathrm{x})=0,
$$

ou seja, $\lim _{m \rightarrow \infty}\left\|s_{m}-f\right\|_{L^{p}\left(\mathbb{S}^{2}\right)}^{p}=0$. Logo dado um $\epsilon>0$, existe $m_{0} \in \mathbb{N}$ tal que

$$
\left\|s_{m}-f\right\|_{L^{p}\left(\mathbb{S}^{2}\right)}^{p}<\epsilon^{p} .
$$

O resultado neste caso segue se escolhemos $s=s_{m_{0}}$, logo $\|s-f\|_{L^{p}\left(\mathbb{S}^{2}\right)}<\epsilon$.

Agora se $f$ é mensurável, então podemos escreve-la como $f=f^{+}-f^{-}$, onde $f^{ \pm}$são funções não negativas mensuráveis. Então, visando o anterior, existem funções simples $s_{1}$ e $s_{2}$ não negativas mensuráveis que se anulam fora de um conjunto de medida finita, tal que,

$$
\left\|f^{+}-s_{1}\right\|<\frac{\epsilon}{2}, \quad \text { e } \quad\left\|f^{-}-s_{2}\right\|<\frac{\epsilon}{2} .
$$


Seja $s=s_{1}-s_{2}$, note-se que $s$ é uma função simples mensurável, que se anula fora de um conjunto de medida finita. Finalmente, pela desigualdade de Minkowski (vide Rudin, 2006) segue

$$
\|f-s\|_{L^{p}\left(\mathbb{S}^{2}\right)}=\left\|\left(f^{+}-s_{1}\right)-\left(f^{-}-s_{2}\right)\right\|_{L^{p}\left(\mathbb{S}^{2}\right)} \leq\left\|f^{+}-s_{1}\right\|_{L^{p}\left(\mathbb{S}^{2}\right)}+\left\|f^{-}-s_{2}\right\|_{L^{p}\left(\mathbb{S}^{2}\right)}<\epsilon .
$$

Isto encerra a demonstração.

São assumidas as Hipóteses A.1 e A.2 e especialmente o esquema ser conservativo.

Demonstração da Proposição 3.5. Com efeito, dada a Hipótese A.2, consideremos o erro de consistência em cada célula de Voronoï por

$$
\widetilde{\xi}_{i}=\left(\mathrm{L}_{s, h}(\widetilde{u})\right)_{i}-f_{i}=\frac{1}{S\left(\widetilde{\mathrm{V}}_{i}\right)} \sum_{j \in \Lambda(i)} m\left(\widetilde{\Gamma}_{i j}\right)\left(-\nabla_{s} u\left(\mathrm{~m}_{i j}\right) \cdot \overrightarrow{\mathrm{n}}_{\mathrm{x}, \widetilde{\Gamma}_{i j}}+\epsilon_{i j}\right)-f_{i} .
$$

Reorganizando os termos acima, podemos escrever

$$
\widetilde{\xi}_{i}=-\frac{1}{S\left(\widetilde{\mathrm{V}}_{i}\right)} \sum_{j \in \Lambda(i)} m\left(\widetilde{\Gamma}_{i j}\right) \nabla_{s} u\left(\mathrm{~m}_{i j}\right) \cdot \overrightarrow{\mathrm{n}}_{\mathrm{x}, \widetilde{\Gamma}_{i j}}-f_{i}+\frac{1}{S\left(\widetilde{\mathrm{V}}_{i}\right)} \sum_{j \in \Lambda(i)} m\left(\widetilde{\Gamma}_{i j}\right) \epsilon_{i j}=\widetilde{\xi}_{i}^{*}+\widetilde{\xi}_{i}^{* *} .
$$

Em virtude da desigualdade triangular temos para $\widetilde{\xi}_{i}^{*}$ que

$$
\begin{aligned}
\left|\widetilde{\xi}_{i}^{*}\right| & =\left|\frac{1}{S\left(\widetilde{\mathrm{V}}_{i}\right)} \sum_{j \in \Lambda(i)} m\left(\widetilde{\Gamma}_{i j}\right) \nabla_{s} u\left(\mathrm{~m}_{i j}\right) \cdot \overrightarrow{\mathrm{n}}_{\mathrm{x}, \widetilde{\Gamma}_{i j}}+f_{i}\right| \\
& \leq\left|\frac{1}{S\left(\widetilde{\mathrm{V}}_{i}\right)} \sum_{j \in \Lambda(i)} m\left(\widetilde{\Gamma}_{i j}\right) \nabla_{s} u\left(\mathrm{~m}_{i j}\right) \cdot \overrightarrow{\mathrm{n}}_{\mathrm{x}, \widetilde{\Gamma}_{i j}}-\frac{1}{S\left(\widetilde{\mathrm{V}}_{i}\right)} \sum_{j \in \Lambda(i)} \mathrm{F}_{i j}^{c}(u)\right|+\left|\frac{1}{S\left(\widetilde{\mathrm{V}}_{i}\right)} \sum_{j \in \Lambda(i)} \mathrm{F}_{i j}^{c}(u)+f_{i}\right| \\
& \leq \frac{1}{S\left(\widetilde{\mathrm{V}}_{i}\right)} \sum_{j \in \Lambda(i)}\left|m\left(\widetilde{\Gamma}_{i j}\right) \nabla_{s} u\left(\mathrm{~m}_{i j}\right) \cdot \overrightarrow{\mathrm{n}}_{\mathrm{x}, \widetilde{\Gamma}_{i j}}-\mathrm{F}_{i j}^{c}(u)\right|+\left|\frac{1}{S\left(\widetilde{\mathrm{V}}_{i}\right)} \sum_{j \in \Lambda(i)} \mathrm{F}_{i j}^{c}(u)+f_{i}\right| .
\end{aligned}
$$

Agora, em vista da Proposição 3.2 e pelo Teorema de Gauss, temos

$$
\begin{aligned}
\left|\widetilde{\xi}_{i}^{*}\right| & \leq C \frac{1}{S\left(\widetilde{\mathrm{V}}_{i}\right)} \sum_{j \in \Lambda(i)} h^{3}+\left|\frac{1}{S\left(\widetilde{\mathrm{V}}_{i}\right)} \sum_{j \in \Lambda(i)} \mathrm{F}_{i j}^{c}(u)+f_{i}\right| \\
& \leq C \frac{1}{S\left(\widetilde{\mathrm{V}}_{i}\right)} \sum_{j \in \Lambda(i)} h^{3}+\left|\frac{1}{S\left(\widetilde{\mathrm{V}}_{i}\right)} \sum_{j \in \Lambda(i)} \int_{\widetilde{\Gamma}_{i j}} \nabla_{s} u(\mathrm{x}) \cdot \overrightarrow{\mathrm{n}}_{\mathrm{x}, \widetilde{\Gamma}_{i j}} d \gamma(\mathrm{x})-f_{i}\right| \\
& \leq C \frac{1}{S\left(\widetilde{\mathrm{V}}_{i}\right)} \sum_{j \in \Lambda(i)} h^{3}+\left|\frac{1}{S\left(\widetilde{\mathrm{V}}_{i}\right)} \int_{\partial \widetilde{\mathrm{V}}_{i}} \nabla_{s} u(\mathrm{x}) \cdot \overrightarrow{\mathrm{n}}_{\mathrm{x}, \widetilde{\mathrm{V}}_{i}} d \gamma(\mathrm{x})-f_{i}\right| \\
& \leq C \frac{1}{S\left(\widetilde{\mathrm{V}}_{i}\right)} \sum_{j \in \Lambda(i)} h^{3}+\left|\frac{1}{S\left(\widetilde{\mathrm{V}}_{i}\right)} \int_{\widetilde{\mathrm{V}}_{i}} \Delta_{s} u(\mathrm{x}) d s(\mathrm{x})-f_{i}\right| .
\end{aligned}
$$

Da Hipótese A.1, ou de maneira mais precisa, existem constantes $C \in \mathbb{R}_{+}$, tal que $C h^{2} \leq S\left(\widetilde{\mathrm{V}}_{i}\right)$ e $\operatorname{Card}\left(\widetilde{\mathrm{V}}_{i}\right) \leq C$, tem-se

$$
\left|\widetilde{\xi}_{i}^{*}\right| \leq C h+\frac{1}{S\left(\widetilde{\mathrm{V}}_{i}\right)}\left|\int_{\widetilde{\mathrm{V}}_{i}} \Delta_{s} u(\mathrm{x}) d s(\mathrm{x})-\int_{\widetilde{\mathrm{V}}_{i}} f(\mathrm{x}) d s(\mathrm{x})\right| \leq C h .
$$

Observe que, no segundo termo do lado direito utilizamos a definição de $f_{i}$, junto à equação (3.1). Portanto,

$$
\left\|\widetilde{\xi}_{h}^{*}\right\|_{\infty, h} \longrightarrow 0, \quad \text { quando } h \longrightarrow 0 \text {. }
$$


Resta provar uma limitação para $\widetilde{\xi}_{i}^{* *}$. Sabemos que este termo está relacionado ao erro $\epsilon_{i j}$ dos fluxos em cada aresta dual, pela Hipótese A.2. Note-se que as células, sob a Hipótese A.1 e o fato de $\left|\epsilon_{i j}\right| \leq C h$, implicam que $\widetilde{\xi}_{i}^{* *}$ não tenda para zero quando $h$ vai para zero. Apesar disso, este fato pode ser corrigido da seguinte maneira: sabemos que

$$
\left|\widetilde{\xi}_{i}^{* *}\right| \leq \frac{1}{S\left(\widetilde{\mathrm{V}}_{i}\right)} \sum_{j \in \Lambda(i)} m\left(\widetilde{\Gamma}_{i j}\right)\left|\epsilon_{i j}\right| \leq C .
$$

Consequentemente, o termo $\left\|\widetilde{\xi}_{h}^{* *}\right\|_{\infty, h}$ é limitado, i.e., existe uma constante $C \in \mathbb{R}_{+}$, independente de $h$, tal que $\left\|\widetilde{\xi}_{i}^{* *}\right\|_{\infty, h}<C$. A demonstrar que a função constante por pedaços $\widetilde{\xi}_{h}^{* *}$ vai para zero no espaço $L^{\infty}\left(\mathbb{S}^{2}\right)$ para a topologia fraca $\star$, ou seja satisfaz o critério de dualidade

$$
\int_{\mathbb{S}^{2}} \widetilde{\xi}_{h}^{* *}(\mathrm{x}) v(\mathrm{x}) d s(\mathrm{x}) \longrightarrow 0, \quad \text { quando } h \longrightarrow 0, \quad \text { para cada } v \in L^{1}\left(\mathbb{S}^{2}\right) .
$$

Cabe agora as observações, em primeiro lugar note que a partir que $\widetilde{\xi}_{i}^{* *}$ ser limitado em $L^{\infty}\left(\mathbb{S}^{2}\right)$, existe uma subsequencia que denotaremos também por $\widetilde{\xi}_{i}^{* *}$, que converge para um limite $\xi^{* *}$ em $L^{\infty}\left(\mathbb{S}^{2}\right)$ para a topologia fraca $\star$. Vamos provar então o critério acima, sejam $v \in C^{\infty}\left(\mathbb{S}^{2}\right)$ e a sua aproximação $v_{h}(\mathrm{x})=v\left(\mathrm{x}_{i}\right)$ para cada $\mathrm{x} \in \widetilde{\mathrm{V}}_{i}$, i.e., constante por pedaços, cujos valores são definidos por

$$
v\left(\mathrm{x}_{i}\right)=\frac{1}{S\left(\widetilde{\mathrm{V}}_{i}\right)} \int_{\widetilde{\mathrm{V}}_{i}} v(\mathrm{x}) d s(\mathrm{x})
$$

Desta maneira, obtemos

$$
\begin{aligned}
\int_{\mathbb{S}^{2}} \widetilde{\xi}_{h}^{* *}(\mathrm{x}) v_{h}(\mathrm{x}) d s(\mathrm{x})=\sum_{i=1}^{N} S\left(\widetilde{\mathrm{V}}_{i}\right) \tilde{\xi}_{i}^{* *} v\left(\mathrm{x}_{i}\right) & =\sum_{i=1}^{N} S\left(\widetilde{\mathrm{V}}_{i}\right)\left[\frac{1}{S\left(\widetilde{\mathrm{V}}_{i}\right)} \sum_{j \in \Lambda(i)} m\left(\widetilde{\Gamma}_{i j}\right) \epsilon_{i j}\right] v\left(\mathrm{x}_{i}\right) \\
& =\sum_{i=1}^{N} \sum_{j \in \Lambda(i)} m\left(\widetilde{\Gamma}_{i j}\right) \epsilon_{i j} v\left(\mathrm{x}_{i}\right) .
\end{aligned}
$$

Agora bem, o esquema é conservativo. Sabemos que os fluxos discretos satisfazem $\mathrm{F}_{i j}^{d}=-\mathrm{F}_{j i}^{d}$, se $\mathrm{x}_{i}$ e $\mathrm{x}_{j}$ são vizinhos com $m\left(\widetilde{\Gamma}_{i j}\right)>0$, então segue a expressão

$$
\sum_{i=1}^{N} \sum_{j \in \Lambda(i)} \mathrm{F}_{i j}^{d}(\widetilde{u}) v\left(\mathrm{x}_{i}\right)=-\sum_{i=1}^{N} \sum_{j \in \Lambda(i)} \mathrm{F}_{j i}^{d}(\widetilde{u}) v\left(\mathrm{x}_{i}\right) .
$$

Com uma troca conveniente dos índices no lado direito, podemos simplificar à relação

$$
\sum_{i=1}^{N} \sum_{j \in \Lambda(i)} \mathrm{F}_{i j}^{d}(\widetilde{u}) v\left(\mathrm{x}_{i}\right)=\frac{1}{2} \sum_{i=1}^{N} \sum_{j \in \Lambda(i)} \mathrm{F}_{i j}^{d}(\widetilde{u})\left[v\left(\mathrm{x}_{j}\right)-v\left(\mathrm{x}_{i}\right)\right] .
$$

Com isto em mente, voltamos à nosso cálculo, para obter

$$
\int_{\mathbb{S}^{2}} \widetilde{\xi}_{h}^{* *}(\mathrm{x}) v_{h}(\mathrm{x}) d s(\mathrm{x})=\frac{1}{2} \sum_{i=1}^{N} \sum_{j \in \Lambda(i)} m\left(\widetilde{\Gamma}_{i j}\right) \epsilon_{i j}\left[v\left(\mathrm{x}_{j}\right)-v\left(\mathrm{x}_{i}\right)\right] .
$$

Lembremos que existem constantes positivas $C \in \mathbb{R}_{+}$, independentes do parâmetro $h$, que implicam $m\left(\widetilde{\Gamma}_{i j}\right) \leq C h$ e $\left|\epsilon_{i j}\right| \leq C h$. Além disso, da escolha da função $v \in C^{\infty}\left(\mathbb{S}^{2}\right)$, temos que as suas primeiras derivadas são contínuas e limitadas, então, em virtude da desigualdade do valor médio, 
existe uma constante $C \in \mathbb{R}_{+}$, independente do parâmetro $h$, tal que $\left|v\left(\mathrm{x}_{j}\right)-v\left(\mathrm{x}_{i}\right)\right| \leq C h$. Logo,

$$
\int_{\mathbb{S}^{2}} \widetilde{\xi}_{h}^{* *}(\mathrm{x}) v_{h}(\mathrm{x}) d s(\mathrm{x}) \leq C \sum_{i=1}^{N} \sum_{j \in \Lambda(i)} h^{3} .
$$

O número total de arestas na decomposição dual é pelo menos de ordem de $h^{-2}$, assim

$$
\int_{\mathbb{S}^{2}} \widetilde{\xi}_{h}^{* *}(\mathrm{x}) v_{h}(\mathrm{x}) d s(\mathrm{x}) \longrightarrow 0, \quad \text { quando } h \longrightarrow 0 .
$$

Agora note-se, que tanto $v$ como sua aproximação $v_{h}$ pertencem ao espaço $L^{1}\left(\mathbb{S}^{2}\right)$. Em particular, $v_{h}$ é uma função simples, ou seja é uma combinação linear de funções características das células de Voronoï, i.e.,

$$
v_{h}(\mathrm{x})=\sum_{i=1}^{N} v\left(\mathrm{x}_{i}\right) \chi_{i}(\mathrm{x})
$$

onde $\chi_{i}$ é a função característica da célula $\widetilde{V}_{i}$. Sabemos que o conjunto de funções simples é denso no espaço $L^{1}\left(\mathbb{S}^{2}\right)$ (vide Lema A.1), logo $v_{h} \longrightarrow v$ pontualmente em $\mathbb{S}^{2}$, quando $h \longrightarrow 0$ e portanto conclua-se que $v_{h}$ converge para $v$ na $L^{1}$-norma, i.e.,

$$
\left\|v_{h}-v\right\|_{L^{1}\left(\mathbb{S}^{2}\right)} \longrightarrow 0, \quad \text { quando } h \longrightarrow 0 .
$$

Segue imediatamente de (A.14) junto ao fato de $\widetilde{\xi}_{h}^{* *} \longrightarrow \xi^{* *}$ em $L^{\infty}\left(\mathbb{S}^{2}\right)$ para a topologia fraca $\star$, que

$$
\int_{\mathbb{S}^{2}} \widetilde{\xi}_{h}^{* *}(\mathrm{x}) v_{h}(\mathrm{x}) d s(\mathrm{x}) \longrightarrow \int_{\mathbb{S}^{2}} \xi^{* *}(\mathrm{x}) v(\mathrm{x}) d s(\mathrm{x}), \quad \text { quando } h \longrightarrow 0 .
$$

Pela unicidade do limite e a expressão (A.13) temos

$$
\int_{\mathbb{S}^{2}} \xi^{* *}(\mathrm{x}) v(\mathrm{x}) d s(\mathrm{x})=0, \quad \text { para cada } v \in C^{\infty}\left(\mathbb{S}^{2}\right) .
$$

Observe-se que a função $v$ está finitamente suportada em $\mathbb{S}^{2}$, portanto

$$
\xi^{* *} \equiv 0 .
$$

A estimativa desejada segue então da combinação de (A.12), (A.13) e (A.15).

O anterior mostra que o esquema de volumes finitos é fracamente consistente. Agora, ainda quando temos a estimativa do Teorema A.1, não é suficiente para encontrar uma condição de estabilidade independente do parâmetro $h$. Em consequência, existem certas limitações para obter estimativas de erro na max-norma de forma direta usando técnicas padrão dos MDF. Portanto, recorre-se a enfraquecer a condição de estabilidade através do processo apresentado nos capítulos 3 e 4.

Observação A.1. Podemos estabelecer uma ordem de convergência quando o critério de HR95 é usado na decomposição Voronoï-Delaunay. Com efeito, a expressão do erro de truncamento,

$$
\xi_{i}=\left(\mathrm{L}_{s, h}(\widetilde{u})\right)_{i}-\frac{1}{S\left(\widetilde{\mathrm{V}}_{i}\right)} \sum_{j \in \Lambda(i)} \mathrm{F}_{i j}^{d}(u), \quad \text { para } i=1 \ldots, N
$$

possui neste caso ordem de $h$, i.e., $\left|\xi_{i}\right| \leq C h$ para cada $i=1, \ldots N$. Portanto, para alguma função $u \in C^{2}\left(\mathbb{S}^{2}\right)$ que satisfaz $\int_{\mathbb{S}^{2}} u(\mathrm{x}) d s(\mathrm{x})=0$, é fácil deduzir que existe uma constante $C \in \mathbb{R}_{+}$, tal que

$$
\left\|\xi_{h}\right\|_{\infty, h} \leq C h\|u\|_{C^{2}\left(\mathbb{S}^{2}\right)} .
$$


De forma análoga, para $u \in H^{2}\left(\mathbb{S}^{2}\right) \cap H_{Q}^{1}\left(\mathbb{S}^{2}\right)$, temos para a $L_{h}^{2}$-norma,

$$
\left\|\xi_{h}\right\|_{0, h}=\left(\sum_{i=1}^{N} S\left(\widetilde{\mathrm{V}}_{i}\right)\left|\xi_{i}\right|^{2}\right)^{1 / 2} \leq C h\|u\|_{H^{2}\left(\mathbb{S}^{2}\right)}
$$

onde na última desigualdade usamos um argumento similar ao feito na Proposição 3.5 para a Hessiana de u. Note-se que a limitação desejada também é consequência direta da expansão em série de Taylor e o argumento de densidade. É importante notar que a estimativa acima não é suficiente para a ordem de convergência dada nos testes numéricos. 
APÊNDICE A 


\section{Apêndice B}

\section{Elementos de cálculo e geometria esférica}

Neste apêndice expomos brevemente sem provas, uma revisão estruturada de noções do cálculo e geometria esférica.

\section{B.1 Fórmula de Taylor e desigualdade de valor médio}

Coletamos um pequeno número de definições básicas do cálculo em várias variáveis no $\mathbb{R}^{3}$ que são usuais nas provas de alguns resultados. Inicialmente recordemos a definição e as propriedades mais simples do desenvolvimento de Taylor para funções de $n$ variáveis. Se $f(\mathrm{x})$ tem $\alpha$-ésima derivada em $\mathrm{x}_{0} \in \mathbb{R}^{n}$, definimos a fórmula de Taylor de grau $\alpha$ de $f$ em torno do ponto $\mathrm{x}_{0}$ em função das diferenciais da seguinte maneira:

$$
f\left(\mathrm{x}_{0}\right)+D_{\mathrm{x}_{0}} f\left(\mathrm{x}-\mathrm{x}_{0}\right)+\frac{1}{2 !} D_{\mathrm{x}_{0}}^{2} f\left(\mathrm{x}-\mathrm{x}_{0}\right)+\cdots-\frac{1}{\alpha !} D_{\mathrm{x}_{0}}^{\alpha} f\left(\mathrm{x}-\mathrm{x}_{0}\right),
$$

onde $D_{\mathrm{x}_{0}}^{\alpha} f(\mathrm{x})$ é dado como

$$
D_{\mathrm{x}_{0}}^{\alpha} f(\mathrm{x})=\sum_{|\alpha|}\left(\begin{array}{c}
\alpha \\
\alpha_{1} \ldots \alpha_{n}
\end{array}\right) x_{1}^{\alpha_{1}} \ldots x_{n}^{\alpha_{n}} \frac{\partial^{\alpha} f}{\partial x_{1}^{\alpha_{1}} \ldots \partial x_{n}^{\alpha_{n}}}\left(\mathrm{x}_{0}\right) .
$$

Teorema B.1 (Fórmula de Taylor). Seja $f: \Omega \subset \mathbb{R}^{n} \rightarrow \mathbb{R}$ uma função de classe $C^{\alpha}$, para $N \in \mathbb{N}$, logo se $\mathrm{x}, \mathrm{y} \in \Omega,[\mathrm{x}, \mathrm{y}]=\{\mathrm{x}+$ ty $: t \in[0,1]\} \subset \Omega$, então

$$
f(\mathrm{x}+\mathrm{y})=\sum_{|\alpha|<N} \frac{1}{\alpha !}\left(\partial_{\mathrm{x}}^{\alpha} f\right)(\mathrm{x}) \mathrm{y}^{\alpha}+N \sum_{|\alpha|=N} \frac{\mathrm{y}^{\alpha}}{\alpha !} \int_{0}^{1}(1-t)^{1-N}\left(\partial_{\mathrm{x}}^{\alpha} f\right)(\mathrm{x}+t \mathrm{y}) d t .
$$

Teorema B.2 (Desigualdade do valor médio). Dado $\Omega \subset \mathbb{R}^{n}$ aberto, seja $f: \Omega \rightarrow \mathbb{R}^{n}$, diferenciável em cada ponto do segmento de reta aberto $(\mathrm{x}, \mathrm{x}+\mathrm{y})$ e tal que sua restrição ao segmento fechado $[\mathrm{x}, \mathrm{x}+\mathrm{y}] \subset \Omega$ seja contínua. Se $\left|f^{\prime}(\mathrm{z})\right| \leq M$ para cada $\mathrm{z} \in(\mathrm{x}, \mathrm{x}+\mathrm{y})$, então $|f(\mathrm{x}+\mathrm{y})-f(\mathrm{x})| \leq M \cdot \mathrm{y}$.

\section{B.2 Coordenadas geográficas}

As coordenadas cartesianas de um ponto $\mathrm{x}=\left(x_{1}, x_{2}, x_{3}\right) \in \mathbb{S}^{2}$ são denotadas nas coordenadas angulares ou geográficas (latitude-longitude),

$$
\mathrm{x}(\phi, \theta)=(\cos (\phi) \cos (\theta), \cos (\phi) \sin (\theta), \sin (\phi)),
$$

onde $\phi \in[-\pi / 2, \pi / 2]$ é a latitude e $\theta \in[-\pi, \pi]$ é a longitude. Por outro lado, denotamos por $d \tilde{s}(\mathrm{x})$ a medida na superfície esférica, a qual admite a representação $d \tilde{s}(\mathrm{x})=\sin (\phi) d \phi d \theta$.

De forma análoga, dado um ponto $\mathrm{x}=\left(x_{1}, x_{2}, x_{3}\right) \in \mathbb{R}^{3}$, as suas coordenadas geográficas são 
dadas por

$$
\begin{aligned}
r & =\sqrt{x_{1}^{2}+x_{2}^{2}+x_{3}^{2}}, \\
\theta & = \begin{cases}\arctan \left(\frac{x_{2}}{x_{1}}\right), & \text { se } x_{1} \neq 0, \\
0, & \text { se } x_{1}=0 .\end{cases} \\
\phi & =\arcsin \left(\frac{x_{3}}{r}\right) .
\end{aligned}
$$

\section{B.3 Distâncias geodésicas}

Para dois pontos em coordenadas geográficas $\mathrm{x}\left(\phi_{1}, \theta_{1}\right)$ e $\mathrm{y}\left(\phi_{2}, \theta_{2}\right)$ usamos a fórmula de Vincenty para determinar a distância geodésica dada como

$$
\mathrm{d}(\mathrm{x}, \mathrm{y})=\left(\frac{\sqrt{\left(\cos \left(\phi_{2}\right) \sin \left(\theta_{1}-\theta_{2}\right)\right)^{2}+\left(\cos \left(\phi_{1}\right) \sin \left(\phi_{2}\right)-\sin \left(\phi_{1}\right) \cos \left(\phi_{2}\right) \cos \left(\theta_{1}-\theta_{2}\right)\right)^{2}}}{\sin \left(\phi_{1}\right) \sin \left(\phi_{2}\right)+\cos \left(\phi_{1}\right) \cos \left(\phi_{2}\right) \cos \left(\theta_{1}-\theta_{2}\right)}\right) .
$$

O cálculo de distância geodésica entre dois pontos x e y na esfera unitária $\mathbb{S}^{2}$ em coordenadas cartesianas é dado pela equação

$$
\mathrm{d}(\mathrm{x}, \mathrm{y})=\arccos \left((\mathrm{x}, \mathrm{y})_{\mathbb{R}^{3}}\right),
$$

mas para evitar problemas na inversa da função cosseno, utilizamos como sugerido por Peixoto (2013), mais precisamente a equação

$$
\mathrm{d}(\mathrm{x}, \mathrm{y})=2 \arctan \left(\sqrt{\frac{4-\|\mathrm{x}+\mathrm{y}\|_{\mathbb{R}^{3}}^{2}}{\|\mathrm{x}+\mathrm{y}\|_{\mathbb{R}^{3}}^{2}}}\right),
$$

onde $\|\cdot\|_{\mathbb{R}^{3}}$ denota a norma Euclidiana.

\section{B.4 Áreas geodésicas}

As áreas dos triângulos esféricos podem ser calculadas com base no excesso esférico obtido pelo Teorema de L'Huilier.

Teorema B.3 (L'Huilier). Seja $\widetilde{\mathrm{T}}$ um triângulo esférico com arestas de cumprimento $\widetilde{\tau}_{1}, \widetilde{\tau}_{2}$ e $\widetilde{\tau}_{3}$ e semi-perímetro s. Então o excesso esférico E é dado por

$$
\tan \left(\frac{1}{4} E\right)=\sqrt{\tan \left(\frac{1}{2} s\right) \tan \left[\frac{1}{2}\left(s-\widetilde{\tau}_{1}\right)\right] \tan \left[\frac{1}{2}\left(s-\widetilde{\tau}_{2}\right)\right] \tan \left[\frac{1}{2}\left(s-\widetilde{\tau}_{3}\right)\right]},
$$

onde $s=\frac{1}{2}\left(\widetilde{\tau}_{1}+\widetilde{\tau}_{2}+\widetilde{\tau}_{3}\right)$.

\section{B.5 Mudança de variável}

Estabelecemos algumas relações uteis para o cálculo de integrais em várias variáveis. Se $\mathbf{A}$ é uma matriz ortogonal no espaço de matrizes $\mathbb{R}^{3 \times 3}$ e $\mathrm{y}=\mathbf{A x}$ para $\mathrm{x}, \mathrm{y} \in \mathbb{S}^{2}$, então $d s(\mathrm{y})=d s(\mathbf{A x})=$ $d s(\mathrm{x})$. Em particular, temos a mudança de um vetor entre o sistema de coordenadas geográficas e cartesiano. Seja $\mathrm{x}=\left(x_{1}, x_{2}, x_{3}\right)$ um ponto na esfera, e seja $\vec{v}=\left(v_{1}, v_{2}, v_{3}\right)=v_{1} \mathbf{e}_{1}+v_{2} \mathbf{e}_{2}+v_{3} \mathbf{e}_{3}$ um vetor nesse ponto, onde $\mathbf{e}_{n}$, com $n \in\{1,2,3\}$ é o vetor da base canônica no $\mathbb{R}^{3}$. A transformação para as coordenadas geográficas, como sendo um vetor $\vec{v}=v_{r} \vec{r}+v_{\phi} \vec{\phi}+v_{\theta} \vec{\theta}$, onde $\vec{r}, \vec{\phi}$ e $\vec{\theta}$ são os vetores canônicos locais em coordenadas geográficas, pode ser feita com a transposta da matriz

$$
\mathbf{A}=\left(\begin{array}{ccc}
\cos (\phi) \cos (\theta) & -\sin (\phi) \cos (\theta) & -\sin (\theta) \\
\cos (\phi) \sin (\theta) & -\sin (\phi) \sin (\theta) & \cos (\theta) \\
\sin (\phi) & \cos (\phi) & 0
\end{array}\right)=\left(\begin{array}{ccc}
\frac{x_{1}}{r} & \frac{x_{3} x_{1}}{\varrho r} & -\frac{x_{2}}{\varrho} \\
\frac{x_{2}}{r} & -\frac{x_{3} x_{2}}{\varrho} & \frac{x_{1}}{\varrho} \\
\frac{x_{3}}{r} & \frac{\varrho}{r} & 0
\end{array}\right)
$$


onde $\varrho=\sqrt{x_{1}^{2}+x_{2}^{2}}$. Para $\mathbf{A}$ ortogonal, temos que a sua inversa é a transposta $\mathbf{A}^{t}$. Então

$$
\left(\begin{array}{l}
v_{1} \\
v_{2} \\
v_{3}
\end{array}\right)=\mathbf{A}\left(\begin{array}{c}
v_{r} \\
v_{\phi} \\
v_{\theta}
\end{array}\right), \quad\left(\begin{array}{l}
v_{r} \\
v_{\phi} \\
v_{\theta}
\end{array}\right)=\mathbf{A}^{t}\left(\begin{array}{l}
v_{1} \\
v_{2} \\
v_{3}
\end{array}\right)
$$

\section{B.5.1 Operadores diferenciais}

A base $\vec{\phi}$ e $\vec{\theta}$ define uma sistema de coordenadas local, que depende do ponto na esfera. Para uma função escalar $u$ definida em coordenadas geográficas, o seu gradiente nessas mesmas coordenadas pode ser calculado da seguinte forma

$$
\nabla_{s} u(\phi, \theta)=\left(\frac{\partial u}{\partial \phi}(\phi, \theta), \frac{1}{\cos (\phi)} \frac{\partial}{\partial \theta} u(\phi, \theta)\right)
$$

Por fim, podemos definir a equação diferencial parcial elíptica de segunda ordem em termos das coordenadas geográficas por

$$
\Delta_{s} u(\phi, \theta)=\frac{1}{\cos (\phi)}\left(\frac{\partial}{\partial \phi}\left(\cos (\phi) \frac{\partial u}{\partial \phi}(\phi, \theta)\right)\right)+\frac{1}{(\cos (\phi))^{2}}\left(\frac{\partial^{2} u}{\partial \theta^{2}}(\phi, \theta)\right) .
$$


APÊNDICE B 


\section{Apêndice C}

\section{Elementos de álgebra linear}

\section{C.1 Considerações técnicas}

Os experimentos numéricos realizados neste estudo foram feitos principalmente em uma laptop ASUS S451LN com as seguintes especificações:

- Processador: Intel ${ }^{\circledR}$ Core $^{\mathrm{TM}}$ i7-4500U CPU @ $1.80 \mathrm{GHz}$

- Memória: $8 \mathrm{~Gb}$

- Sistema operacional: Ubuntu 16.10 LTS 64 bits

- Compilador: Intel ${ }^{\circledR}$ Fortran Compiler 9.2.1

Os códigos e programas computacionais foram todos desenvolvidos em FORTRAN90 na tese de doutorado de Peixoto (2013). Alguns experimentos que demandavam mais processamento foram realizados em um servidor numérico do laboratório de computação científica em matemática aplicada (LabMap) do IME-USP.

Para o desenvolvimento das figuras foi usado o software GMT - The Generic Mapping Tools data processing and display software, versão 5.4.5 para Linux. Os diagramas bidimensionais de geometria foram feitos no software GeoGebra 4 e o pacote do elementos gráficos tikz do $\mathrm{AT}_{\mathrm{E}} \mathrm{X}$.

\section{C.2 Existência da solução discreta}

A expressão (3.11) forma um sistema linear de equações dado como

$$
\mathbf{L}_{s, h} \mathbf{u}_{h}=\mathbf{f}_{h}
$$

onde a matriz $\mathbf{L}_{s, h}=\left[l_{i j}\right]_{N \times N}$ é uma $N \times N$-matriz, para $N=10 \cdot 2^{2 \ell}+2$, com entradas na diagonal

$$
l_{i i}=\sum_{j \in \Lambda(i)} \frac{m\left(\widetilde{\Gamma}_{i j}\right)}{\left\|\mathrm{x}_{j}-\mathrm{x}_{i}\right\|}, \quad \text { para } j \in \Lambda(i),
$$

e fora da diagonal

$$
l_{i j}= \begin{cases}-\frac{m\left(\widetilde{\Gamma}_{i j}\right)}{\left\|\mathrm{x}_{j}-\mathrm{x}_{i}\right\|}, & \text { para } j \in \Lambda(i) \\ 0, & \text { caso contrário. }\end{cases}
$$

$\mathrm{O}$ vetor $\mathbf{u}_{h}$ tem como componentes os valores da função $u_{h}$ em cada célula de Voronoi $\widetilde{\mathrm{V}}_{i}$, i.e.,

$$
\mathbf{u}_{h}=\left[u_{h}\left(\mathrm{x}_{1}\right), \ldots, u_{h}\left(\mathrm{x}_{N}\right)\right]^{t},
$$

e o vetor $\mathbf{f}_{h}$ é o vetor cujos componentes são os valores médios de $f$ em cada $\widetilde{\mathrm{V}}_{i}$ vezes o valor da área superficial $S\left(\widetilde{\mathrm{V}}_{i}\right)$ da célula respectivamente, i.e.,

$$
\mathbf{f}_{h}=\left[\bar{f}_{1}, \ldots, \bar{f}_{N}\right]^{t}
$$


onde $\bar{f}_{i}=S\left(\widetilde{\mathrm{V}}_{i}\right) f_{i}$ para cada $i=1, \ldots, N$.

A matriz $\mathbf{L}_{s, h}$ é simétrica e satisfaz as condições

$$
l_{i i}>0, \quad l_{i j} \leq 0, \quad \text { para cada } i \neq j, \quad 1 \leq i, j \leq N .
$$

Porém $\mathbf{L}_{s, h}$ é uma matriz singular, i.e., em geral o sistema (C.1) não tem solução. Vejamos algumas características. Em relação à existência da solução, se a condição de compatibilidade

$$
\left\langle\mathbf{f}_{h}, \mathbf{1}\right\rangle_{h}=\sum_{i=1}^{N} S\left(\widetilde{\mathrm{V}}_{i}\right) f_{i}=0,
$$

é satisfeita. O sistema (C.1) pode ser resolvido. Seja $\mathbf{u}_{h}$, então podemos construir uma submatriz $\widetilde{\mathbf{L}}_{s, h}$ eliminando uma linha e uma coluna de $\mathbf{L}_{s, h}$, dado um vértice $\mathrm{x}_{i^{*}} \in \mathrm{S}_{N}=\left\{\mathrm{x}_{i}\right\}_{i=1}^{N}$, normalizamos $\mathbf{u}_{h}$ por $\mathbf{u}_{h}\left(\mathrm{x}_{i^{*}}\right)=0$. Desta maneira, denotamos por $\hat{\mathbf{u}}_{h}$ ao vetor $\mathbf{u}_{h}$ sem a componente $u_{h}\left(\mathrm{x}_{i^{*}}\right)$. Então, obtemos o sistema

$$
\widetilde{\mathbf{L}}_{s, h} \hat{\mathbf{u}}_{h}=\hat{\mathbf{f}}_{h},
$$

com $(N-1)^{2}-1$ equações e incógnitas. Aqui $\hat{\mathbf{f}}_{h}$ representa o vetor $\mathbf{f}_{h}$ sem a componente $f_{i^{*}}$. Considere as seguintes definições.

Definição C.1 (Hackbusch, 2017). A matriz $\widetilde{\mathbf{L}}_{s, h}=\left[l_{i j}\right]_{(N-1) \times(N-1)}$ é dita irredutível se não existir uma matriz de permutação $\mathbf{P}$ tal que

$$
\mathbf{P} \widetilde{\mathbf{L}}_{s, h} \mathbf{P}^{t}=\left[\begin{array}{cc}
\mathbf{A} & \mathbf{B} \\
0 & \mathbf{D}
\end{array}\right]
$$

onde $n<(N-1), \mathbf{A}=\left[a_{i j}\right]_{n \times n}, \mathbf{B}=\left[b_{i j}\right]_{n \times(N-1)-n} e \mathbf{D}=\left[a_{i j}\right]_{((N-1)-n) \times((N-1)-n)}$.

Definição C.2 (Hackbusch, 2017). Seja $N=10 \cdot 2^{2 \ell}+2$. Uma matriz $\widetilde{\mathbf{L}}_{s, h}=\left[l_{i j}\right]_{(N-1) \times(N-1)} e ́$ dita fracamente diagonal dominante se

$$
\left|l_{i i}\right| \geq \sum_{\substack{j=1 \\ j \neq i}}^{N-1} l_{i j}, \quad i=1, \ldots, N-1
$$

e vale a desigualdade estrita para alguma linha.

Teorema C.1 (Hackbusch, 2017). Toda matriz fracamente diagonal dominante e irredutível é inversivel.

Segundo o Teorema 4.7.4 de Hackbusch (2017, pp. 75) temos:

Teorema C.2 (Hackbusch, 2017). O sistema de equações de $\widetilde{\mathbf{L}}_{s, h} \hat{\mathbf{u}}_{h}=\hat{\mathbf{f}}_{h}$ é solucionável; em particular, $\widetilde{\mathbf{L}}_{s, h}$ é fracamente diagonal dominante e irredutivel e portanto não singular. Sob a condição de compatibilidade discreta $\left\langle\hat{\mathbf{f}}_{h}, \mathbf{1}\right\rangle_{h}=0$ e $\hat{\mathbf{u}}_{h}=\widetilde{\mathbf{L}}_{s, h}^{-1} \hat{\mathbf{f}}_{h}$, implicam uma solução $\hat{\mathbf{u}}_{h}$ ao sistema (C.1).

\section{C.3 Método de Gauss-Seidel SOR (Succesive over relaxation)}

O método de relaxação usado é obtido a partir de uma modificação no método de Jacobi. Este esquema é dado como

$$
\widetilde{u}_{i}^{[k+1]}=(1-\omega) \widetilde{u}_{i}^{[k]}+\omega \widetilde{u}_{i}^{*},
$$

onde

$$
\widetilde{u}_{i}^{*}=\left(\sum_{j \in \Lambda(i)} \frac{m\left(\widetilde{\Gamma}_{i j}\right)}{\left\|\mathrm{x}_{i}-\mathrm{x}_{j}\right\|} \widetilde{u}_{i}^{[k]}-S\left(\widetilde{\mathrm{V}}_{i}\right) f_{i}\right)\left(\frac{1}{\sum_{j \in \Lambda(i)} \frac{m\left(\widetilde{\Gamma}_{i j}\right)}{\left\|\mathrm{x}_{i}-\mathrm{x}_{j}\right\|}}\right),
$$


onde $\omega \in \mathbb{R}$ é o fator de relaxação. Para os testes numéricos usamos $\omega=1.7$. O método, na maioria das vezes, têm convergência mais rápida do que o método de usual de Gauss-Seidel, mas exigem processamento serial. 
APÊNDICE C 


\section{Referências Bibliográficas}

Atkinson e Han(2012) Kendall Atkinson e Weimin Han. Spherical harmonics and approximations on the unit sphere: an introduction, volume 2044. Springer Science \& Business Media. Citado na pág. 1

Aubin(2013) Thierry Aubin. Some nonlinear problems in Riemannian geometry. Springer Science \& Business Media. Citado na pág. xviii, 1, 2, 4, 5, 52

Augenbaum e Peskin(1985) Jeffrey M Augenbaum e Charles S Peskin. On the construction of the Voronoi mesh on a sphere. Journal of Computational Physics, 59(2):177-192. Citado na pág. $\mathrm{xv}, 14$

Bank e Rose(1987) Randolph E Bank e Donald J Rose. Some error estimates for the box method. SIAM Journal on Numerical Analysis, 24(4):777-787. Citado na pág. 43

Barbeiro(2009) Silvia Barbeiro. Supraconvergent cell-centered scheme for two dimensional elliptic problems. Applied numerical mathematics, 59(1):56-72. Citado na pág. xvi

Baumgardner e Frederickson(1985) John R Baumgardner e Paul O Frederickson. Icosahedral discretization of the two-sphere. SIAM Journal on Numerical Analysis, 22(6):1107-1115. Citado na pág. Xv, 10

Bonito e Demlow(2019) Andrea Bonito e Alan Demlow. A posteriori error estimates for the Laplace-Beltrami operator on parametric $C^{2}$ surfaces. SIAM J. Numer. Anal., 57(3):973-996. ISSN 0036-1429. doi: 10.1137/18M1169278. URL https://doi.org/10.1137/18M1169278. Citado na pág. xvii, 66

Bouche et al.(2005) Daniel Bouche, Jean-Michel Ghidaglia e Frédéric Pascal. Error estimate and the geometric corrector for the upwind finite volume method applied to the linear advection equation. SIAM J. Numer. Anal., 43(2):578-603. ISSN 0036-1429. doi: 10.1137/040605941. URL https://doi.org/10.1137/040605941. Citado na pág. xvi

Brenner e Scott(2007) Susanne Brenner e Ridgway Scott. The mathematical theory of finite element methods, volume 15. Springer Science \& Business Media. Citado na pág. xvii, 33, 34, 54

Brezis(2010) Haim Brezis. Functional analysis, Sobolev spaces and partial differential equations. Springer Science \& Business Media. Citado na pág. 6, 71, 73

Cai(1990) Zhiqiang Cai. On the finite volume element method. Numerische Mathematik, 58(1): 713-735. Citado na pág. xvii

Chen et al.(2002) Zhongying Chen, Ronghua Li e Aihui Zhou. A note on the optimal $L^{2}$-estimate of the finite volume element method. Advances in computational Mathematics, 16(4):291-303. Citado na pág. 65

Chou e Li(2000) So-Hsiang Chou e Qian Li. Error estimates in $L^{2}, H^{1}$ and $L^{\infty}$ in covolume methods for elliptic and parabolic problems: a unified approach. Mathematics of Computation, 69(229):103-120. Citado na pág. xviii, 64, 65 
Chou e Ye(2007) So-Hsiang Chou e Xiu Ye. Superconvergence of finite volume methods for the second order elliptic problem. Computer methods in applied mechanics and engineering, 196(37): 3706-3712. Citado na pág. xviii, 33, 65

Chou et al.(2003) So-Hsiang Chou, Do Y Kwak e Qian Li. $L^{p}$ error estimates and superconvergence for covolume or finite volume element methods. Numerical Methods for Partial Differential Equations, 19(4):463-486. Citado na pág. xviii, 34, 65

Ciarlet(2002) Philippe G Ciarlet. The finite element method for elliptic problems. SIAM. Citado na pág. xvii, 13,34

Coudière et al.(1999) Yves Coudière, Jean-Paul Vila e Philippe Villedieu. Convergence rate of a finite volume scheme for a two dimensional convection-diffusion problem. ESAIM: Mathematical Modelling and Numerical Analysis, 33(3):493-516. Citado na pág. xvi, 21, 28, 47, 67

Demlow(2009) Alan Demlow. Higher-order finite element methods and pointwise error estimates for elliptic problems on surfaces. SIAM Journal on Numerical Analysis, 47(2):805-827. Citado na pág. xv, xvii, xviii, $7,31,32,33,34,52,53,54,64,66$

Demlow e Dziuk(2007) Alan Demlow e Gerhard Dziuk. An adaptive finite element method for the laplace-beltrami operator on implicitly defined surfaces. SIAM Journal on Numerical Analysis, 45(1):421-442. Citado na pág. xv, xvii, 30, 32, 66

Despres(2004) Bruno Despres. Lax theorem and finite volume schemes. Mathematics of computation, 73(247):1203-1234. Citado na pág. xvi

Diskin e Thomas(2010) Boris Diskin e James L. Thomas. Notes on accuracy of finite-volume discretization schemes on irregular grids. Appl. Numer. Math., 60(3):224-226. ISSN 0168-9274. doi: 10.1016/j.apnum.2009.12.001. URL https://doi.org/10.1016/j.apnum.2009.12.001. Citado na pág. xvi, 65

Droniou(2014) Jerome Droniou. Finite volume schemes for diffusion equations: introduction to and review of modern methods. Mathematical Models and Methods in Applied Sciences, 24(08): 1575-1619. Citado na pág. xvi

Du e Gunzburger(2002) Qiang Du e Max Gunzburger. Grid generation and optimization based on centroidal Voronoi tessellations. Applied mathematics and computation, 133(2-3):591-607. Citado na pág. 18

Du e Ju(2005) Qiang Du e Lili Ju. Finite volume methods on spheres and spherical centroidal Voronoi meshes. SIAM Journal on Numerical Analysis, 43(4):1673-1692. Citado na pág. xviii, 28, $30,31,36,39,45,48,49,51,64,65$

Du e Wang(2006) Qiang Du e Desheng Wang. Recent progress in robust and quality delaunay mesh generation. Journal of Computational and Applied Mathematics, 195(1-2):8-23. Citado na pág. XV

Du et al.(2003a) Qiang Du, Max D Gunzburger e Lili Ju. Constrained centroidal Voronoi tessellations for surfaces. SIAM Journal on Scientific Computing, 24(5):1488-1506. Citado na pág. ix, xvi, 17, 18, 19, 65

Du et al.(2003b) Qiang Du, Max D Gunzburger e Lili Ju. Voronoi-based finite volume methods, optimal Voronoi meshes, and PDEs on the sphere. Computer methods in applied mechanics and engineering, 192(35):3933-3957. Citado na pág. xvi, xvii, 16, 17, 18, 28, 31, 36, 45, 64, 65, 67, 70, 72 
Du et al.(2010) Qiang Du, Max Gunzburger e Lili Ju. Advances in studies and applications of centroidal Voronoi tessellations. Numerical Mathematics: Theory, Methods and Applications, 3 (2):119-142. Citado na pág. 18

Dziuk(1988) Gerhard Dziuk. Finite elements for the Beltrami operator on arbitrary surfaces. Em Partial differential equations and calculus of variations, páginas 142-155. Springer. Citado na pág. xvii

Dziuk e Elliott(2013) Gerhard Dziuk e Charles M Elliott. Finite element methods for surface pdes. Acta Numerica, 22:289-396. Citado na pág. xv, xvii, 2, 4, 7, 30, 31, 32

Ewing et al.(2002) Richard E Ewing, Tao Lin e Yanping Lin. On the accuracy of the finite volume element method based on piecewise linear polynomials. SIAM Journal on Numerical Analysis, 39(6):1865-1888. Citado na pág. xvii, xviii, 45, 48, 64, 65, 66

Eymard et al.(2006) R. Eymard, T. Gallouët e R. Herbin. A cell-centered finite-volume approximation for anisotropic diffusion operators on unstructured meshes in any space dimension. IMA J. Numer. Anal., 26(2):326-353. ISSN 0272-4979. doi: 10.1093/imanum/dri036. URL https://doi.org/10.1093/imanum/dri036. Citado na pág. xvi, 47

Eymard et al.(2000) Robert Eymard, Thierry Gallouët e Raphaèle Herbin. Finite volume methods. Handbook of numerical analysis, 7:713-1018. Citado na pág. xvi, 21, 47, 67, 68

Eymard et al.(2001) Robert Eymard, Thierry Gallouët e Raphaèle Herbin. Finite volume approximation of elliptic problems and convergence of an approximate gradient. Applied Numerical Mathematics, 37(1-2):31-53. Citado na pág. xvi, xvii, 28, 45

Faille(1992) Isabelle Faille. A control volume method to solve an elliptic equation on a twodimensional irregular mesh. Computer methods in applied mechanics and engineering, 100(2): 275-290. Citado na pág. xvii, 21, 28, 67

Gallouët et al.(2000) Thierry Gallouët, Raphaele Herbin e Marie Hélene Vignal. Error estimates on the approximate finite volume solution of convection diffusion equations with general boundary conditions. SIAM Journal on Numerical Analysis, 37(6):1935-1972. Citado na pág. xvi, xvii, $28,45,47,64,67$

Gärtner e Kamenski(2019) K. Gärtner e L. Kamenski. Why Do We Need Voronoi Cells and Delaunay Meshes? Essential Properties of the Voronoi Finite Volume Method. Comput. Math. Math. Phys., 59(12):1930-1944. ISSN 0965-5425. doi: 10.1134/S096554251912008X. URL https://doi.org/10.1134/S096554251912008X. Citado na pág. xv, xvi, 13

Giraldo(1997) Francis X Giraldo. Lagrange-Galerkin methods on spherical geodesic grids. Journal of Computational Physics, 136(1):197-213. Citado na pág. xv, 10

Giraldo(2000) Francis X Giraldo. Lagrange-Galerkin methods on spherical geodesic grids: the shallow water equations. Journal of Computational Physics, 160(1):336-368. Citado na pág. 10

Gregory et al.(2008) Matthew J Gregory, A Jon Kimerling, Denis White e Kevin Sahr. A comparison of intercell metrics on discrete global grid systems. Computers, Environment and Urban Systems, 32(3):188-203. Citado na pág. 11, 65

Hackbusch(1989) W. Hackbusch. On first and second order box schemes. Computing, 41(4):277296. ISSN 0010-485X. doi: 10.1007/BF02241218. URL https://doi.org/10.1007/BF02241218. Citado na pág. 43

Hackbusch(2017) Wolfgang Hackbusch. Elliptic differential equations: theory and numerical treatment, volume 18. Springer. Citado na pág. 28, 84 
Harris et al.(2011) Lucas M Harris, Peter H Lauritzen e Rashmi Mittal. A flux-form version of the conservative semi-Lagrangian multi-tracer transport scheme (CSLAM) on the cubed sphere grid. Journal of Computational Physics, 230(4):1215-1237. Citado na pág. 10

Hebey(1996) Emmanuel Hebey. Sobolev spaces on Riemannian manifolds, volume 1635. Springer Science \& Business Media. Citado na pág. xviii, 1, 4, 5, 71

Heikes e Randall(1995a) Ross Heikes e David A Randall. Numerical integration of the shallowwater equations on a twisted icosahedral grid. Part I: Basic design and results of tests. Monthly Weather Review, 123(6):1862-1880. Citado na pág. xv, xvi, 10

Heikes e Randall(1995b) Ross Heikes e David A Randall. Numerical integration of the shallowwater equations on a twisted icosahedral grid. Part II. A detailed description of the grid and an analysis of numerical accuracy. Monthly Weather Review, 123(6):1881-1887. Citado na pág. ix, xvi, xvii, 17, 19, 21, 24, 25, 26, 27, 29, 61, 65

Heikes et al.(2013) Ross P Heikes, David A Randall e Celal S Konor. Optimized icosahedral grids: Performance of finite-difference operators and multigrid solver. Monthly Weather Review, 141(12):4450-4469. Citado na pág. 26

Heinze e Hense(2002) Th Heinze e A Hense. The shallow water equations on the sphere and their Lagrange-Galerkin-solution. Meteorology and Atmospheric Physics, 81(1-2):129-135. Citado na pág. $\mathrm{xv}, 9,10$

Herbin(1995) Raphaèle Herbin. An error estimate for a finite volume scheme for a diffusionconvection problem on a triangular mesh. Numerical Methods for Partial Differential Equations, 11(2):165-173. Citado na pág. xvi, 45

Hermeline(2000) F Hermeline. A finite volume method for the approximation of diffusion operators on distorted meshes. Journal of computational Physics, 160(2):481-499. Citado na pág. xvi

Herrmann et al.(2018) Lukas Herrmann, Annika Lang e Christoph Schwab. Numerical analysis of lognormal diffusions on the sphere. Stochastics and Partial Differential Equations: Analysis and Computations, 6(1):1-44. Citado na pág. 7

Hjelle e Dæhlen(2006) Øyvind Hjelle e Morten Dæhlen. Triangulations and applications. Springer Science \& Business Media. Citado na pág. xvi, 13, 14

Ii e Xiao(2010) Satoshi Ii e Feng Xiao. A global shallow water model using high order multimoment constrained finite volume method and icosahedral grid. Journal of Computational physics, 229(5):1774-1796. Citado na pág. xv

Jianguo e Shitong(1998) Huang Jianguo e Xi Shitong. On the finite volume element method for general self-adjoint elliptic problems. SIAM journal on numerical analysis, 35(5):1762-1774. Citado na pág. xviii, 65

Ju e Du(2009) Lili Ju e Qiang Du. A finite volume method on general surfaces and its error estimates. J. Math. Anal. Appl., 352(2):645-668. ISSN 0022-247X. doi: 10.1016/j.jmaa.2008.11. 022. URL https://doi.org/10.1016/j.jmaa.2008.11.022. Citado na pág. xvii, xviii, 19, 30, 32, 45, 64

Ju et al.(2002) Lili Ju, Qiang Du e Max Gunzburger. Probabilistic methods for centroidal Voronoi tessellations and their parallel implementations. Parallel Computing, 28(10):1477-1500. Citado na pág. 18

Ju et al.(2009) Lili Ju, Li Tian e Desheng Wang. A posteriori error estimates for finite volume approximations of elliptic equations on general surfaces. Comput. Methods Appl. Mech. Engrg., 198(5-8):716-726. ISSN 0045-7825. doi: 10.1016/j.cma.2008.10.007. URL https://doi.org/10.1016/j.cma.2008.10.007. Citado na pág. xvii, xviii, 30, 32, 66 
Kovács e Power Guerra(2018) Balázs Kovács e Christian Andreas Power Guerra. Maximum norm stability and error estimates for the evolving surface finite element method. Numerical Methods for Partial Differential Equations, 34(2):518-554. Citado na pág. xviii, 66

Kreiss et al.(1986) H-O Kreiss, Thomas A Manteuffel, B Swartz, B Wendroff e AB White. Supraconvergent schemes on irregular grids. Mathematics of Computation, 47(176):537-554. Citado na pág. xvi, 65

Kröner(2017) Heiko Kröner. Approximative Green's functions on surfaces and pointwise error estimates for the finite element method. Comput. Methods Appl. Math., 17(1):51-64. ISSN 16094840. doi: 10.1515/cmam-2016-0036. URL https://doi.org/10.1515/cmam-2016-0036. Citado na pág. xvii, xviii, $52,54,66$

LeVeque et al.(2002) Randall J LeVeque et al. Finite volume methods for hyperbolic problems, volume 31. Cambridge university press. Citado na pág. xvi

Li et al.(2000) Ronghua Li, Zhongying Chen e Wei Wu. Generalized difference methods for differential equations: numerical analysis of finite volume methods. CRC Press. Citado na pág. xvii, xviii, 19, 29, 32, 33, 65

Lin et al.(2013) Yanping Lin, Jiangguo Liu e Min Yang. Finite volume element methods: an overview on recent developments. Int. J. Numer. Anal. Model. Ser. B, 4(1):14-34. Citado na pág. xvii, $16,32,65$

Lv e Li(2010) Junliang Lv e Yonghai Li. $L^{2}$ error estimate of the finite volume element methods on quadrilateral meshes. Advances in Computational Mathematics, 33(2):129-148. Citado na pág. 66

Manteuffel e White(1986) Thomas A Manteuffel e Andrew B White. The numerical solution of second-order boundary value problems on nonuniform meshes. Mathematics of Computation, 47(176):511-535. Citado na pág. xvi, 65

Mishev(1998) Ilya D Mishev. Finite volume methods on Voronoi meshes. Numerical methods for Partial Differential equations, 14(2):193-212. Citado na pág. xvi, xvii, 19, 28, 36, 45

Miura e Kimoto(2005) Hiroaki Miura e Masahide Kimoto. A comparison of grid quality of optimized spherical hexagonal-pentagonal geodesic grids. Monthly weather review, 133(10):28172833. Citado na pág. xvi, 10, 17, 18, 65

Morimoto(1998) Mitsuo Morimoto. Analytic functionals on the sphere. American Mathematical Society. Citado na pág. 1, 2, 4

Murray(1996) Ross J Murray. Explicit generation of orthogonal grids for ocean models. Journal of Computational Physics, 126(2):251-273. Citado na pág. 10

Nie et al.(2017) Cunyun Nie, Shi Shu, Haiyuan Yu e Wenhua Xia. Superconvergence and asymptotic expansions for bilinear finite volume element approximation on non-uniform grids. J. Comput. Appl. Math., 321:323-335. ISSN 0377-0427. doi: 10.1016/j.cam.2016.12.024. URL https://doi.org/10.1016/j.cam.2016.12.024. Citado na pág. 66

Omnes(2011) Pascal Omnes. On the second-order convergence of a function reconstructed from finite volume approximations of the Laplace equation on Delaunay-Voronoi meshes. ESAIM: Mathematical Modelling and Numerical Analysis, 45(4):627-650. Citado na pág. xviii

Pascal(2007) Frédéric Pascal. On supra-convergence of the finite volume method for the linear advection problem. Em Paris-Sud Working Group on Modelling and Scientific Computing 20062007, volume 18 of ESAIM Proc., páginas 38-47. EDP Sci., Les Ulis. doi: 10.1051/proc:071804. URL https://doi.org/10.1051/proc:071804. Citado na pág. xvi, 28, 65 
Peixoto(2013) Pedro da Silva Peixoto. Análise de discretizações e interpolações em malhas icosaédricas e aplicações em modelos de transporte semi-lagrangianos. Tese de Doutorado, Universidade de São Paulo. Citado na pág. xvi, 14, 16, 17, 24, 65, 80, 83

Peixoto e Barros(2013) Pedro S Peixoto e Saulo RM Barros. Analysis of grid imprinting on geodesic spherical icosahedral grids. Journal of Computational Physics, 237:61-78. Citado na pág. 24

Putman e Lin(2007) William M Putman e Shian-Jiann Lin. Finite-volume transport on various cubed-sphere grids. Journal of Computational Physics, 227(1):55-78. Citado na pág. 9, 10

Randall et al.(1998) David Randall, Judith Curry, David Battisti, Gregory Flato, Robert Grumbine, Sirpa Hakkinen, Doug Martinson, Ruth Preller, John Walsh e John Weatherly. Status of and outlook for large-scale modeling of atmosphere-ice-ocean interactions in the Arctic. Bulletin of the American Meteorological Society, 79(2):197-219. Citado na pág. xv, 10

Rannacher e Scott(1982) Rolf Rannacher e Ridgway Scott. Some optimal error estimates for piecewise linear finite element approximations. mathematics of computation, 38(158):437-445. Citado na pág. xviii, 53, 54, 66

Renka(1997) Robert J Renka. Algorithm 773: SSRFPACK: interpolation of scattered data on the surface of a sphere with a surface under tension. ACM Transactions on Mathematical Software (TOMS), 23(3):435-442. Citado na pág. xv, 13

Ringler(2003) Todd Ringler. Comparing truncation error to PDE solution error on spherical Voronoi tessellations. Relatório técnico, Tech report, Dept. Atmospheric Sci., Colorado State Univ. Citado na pág. xvii, 16, 17, 21, 27, 65

Ronchi et al.(1996) C Ronchi, R Iacono e Pier S Paolucci. The cubed sphere: a new method for the solution of partial differential equations in spherical geometry. Journal of Computational Physics, 124(1):93-114. Citado na pág. xv, 10

Rudin(2006) Walter Rudin. Real and complex analysis. Tata McGraw-hill education. Citado na pág. 73,74

Sadourny(1972) Robert Sadourny. Conservative finite-difference approximations of the primitive equations on quasi-uniform spherical grids. Monthly Weather Review, 100(2):136-144. Citado na pág. 10

Sadourny et al.(1968) Robert Sadourny, Akio Arakawa e Yale Mintz. Integration of the nondivergent barotropic vorticity equation with an icosahedral-hexagonal grid for the sphere. Monthly Weather Review, 96(6):351-356. Citado na pág. xv, 10

Sahr et al.(2003) Kevin Sahr, Denis White e A Jon Kimerling. Geodesic discrete global grid systems. Cartography and Geographic Information Science, 30(2):121-134. Citado na pág. 10

Satoh(2013) Masaki Satoh. Atmospheric circulation dynamics and general circulation models. Springer Science \& Business Media. Citado na pág. xvi, 10, 12

Schatz e Wahlbin(1995) A. H. Schatz e L. B. Wahlbin. Interior maximum-norm estimates for finite element methods. II. Math. Comp., 64(211):907-928. ISSN 0025-5718. doi: 10.2307/2153476. URL https://doi.org/10.2307/2153476. Citado na pág. 53, 54, 66

Schatz(1998) Alfred Schatz. Pointwise error estimates and asymptotic error expansion inequalities for the finite element method on irregular grids: Part I. Global estimates. Mathematics of Computation of the American Mathematical Society, 67(223):877-899. Citado na pág. 54, 66 
Scott(1976) Ridgway Scott. Optimal $L^{\infty}$ estimates for the finite element method on irregular meshes. Math. Comp., 30(136):681-697. ISSN 0025-5718. Citado na pág. xviii, 54

Scott et al.(2016) RK Scott, LM Harris e LM Polvani. A test case for the inviscid shallowwater equations on the sphere. Quarterly Journal of the Royal Meteorological Society, 142(694): 488-495. Citado na pág. xv, 10

Strikwerda(2004) John C. Strikwerda. Finite difference schemes and partial differential equations. Society for Industrial and Applied Mathematics (SIAM), Philadelphia, PA, second edição. ISBN 0-89871-567-9. doi: 10.1137/1.9780898717938. URL https://doi.org/10.1137/1. 9780898717938. Citado na pág. 27

Thuburn(1997) John Thuburn. A PV-based shallow-water model on a hexagonal-icosahedral grid. Monthly Weather Review, 125(9):2328-2347. Citado na pág. 10

Tomita et al.(2001) Hirofumi Tomita, Motohiko Tsugawa, Masaki Satoh e Koji Goto. Shallow water model on a modified icosahedral geodesic grid by using spring dynamics. Journal of Computational Physics, 174(2):579-613. Citado na pág. xv, xvi, 10, 12, 16

Tomita et al.(2002) Hirofumi Tomita, Masaki Satoh e Koji Goto. An optimization of the icosahedral grid modified by spring dynamics. Journal of Computational Physics, 183(1):307331. Citado na pág. xvi

Vanselow(1996) R Vanselow. Relations between FEM and FVM applied to the Poisson equation. Computing, (57):93-104. Citado na pág. 43

Vreugdenhil(2013) Cornelis Boudewijn Vreugdenhil. Numerical methods for shallow-water flow, volume 13. Springer Science \& Business Media. Citado na pág. xv

Wang e Lee(2011) Ning Wang e Jin-Luen Lee. Geometric properties of the icosahedral-hexagonal grid on the two-sphere. SIAM Journal on Scientific Computing, 33(5):2536-2559. Citado na pág. xvi, 10

Williamson(1968) David L Williamson. Integration of the barotropic vorticity equation on a spherical geodesic grid. Tellus, 20(4):642-653. Citado na pág. xv, 10

Williamson(2007) David L Williamson. The evolution of dynamical cores for global atmospheric models. Journal of the Meteorological Society of Japan, 85:241-269. Citado na pág. 9, 10

Williamson et al.(1992) David L Williamson, John B Drake, James J Hack, Rüdiger Jakob e Paul N Swarztrauber. A standard test set for numerical approximations to the shallow water equations in spherical geometry. Journal of Computational Physics, 102(1):211-224. Citado na pág. xvi

Wu e Li(2003) Haijun Wu e Ronghua Li. Error estimates for finite volume element methods for general second-order elliptic problems. Numerical Methods for Partial Differential Equations, 19 (6):693-708. Citado na pág. xviii

$\mathbf{X u}(2006)$ Guoliang Xu. Discrete Laplace-Beltrami operator on sphere and optimal spherical triangulations. International Journal of Computational Geometry \& Applications, 16(01):75-93. Citado na pág. 24

Zhang(2014) Tie Zhang. Superconvergence of finite volume element method for elliptic problems. Advances in Computational Mathematics, 40(2):399-413. Citado na pág. 29, 34, 64, 65 